DOE/LLW-107

Distribution Category: UC-512

DOE/LLW--107

DE9 I 006401

\title{
1989 STATE-BY-STATE ASSESSMENT OF LOW-LEVEL RADIOACTIVE WASTES RECEIVED AT COMMERCIAL DISPOSAL SITES
}

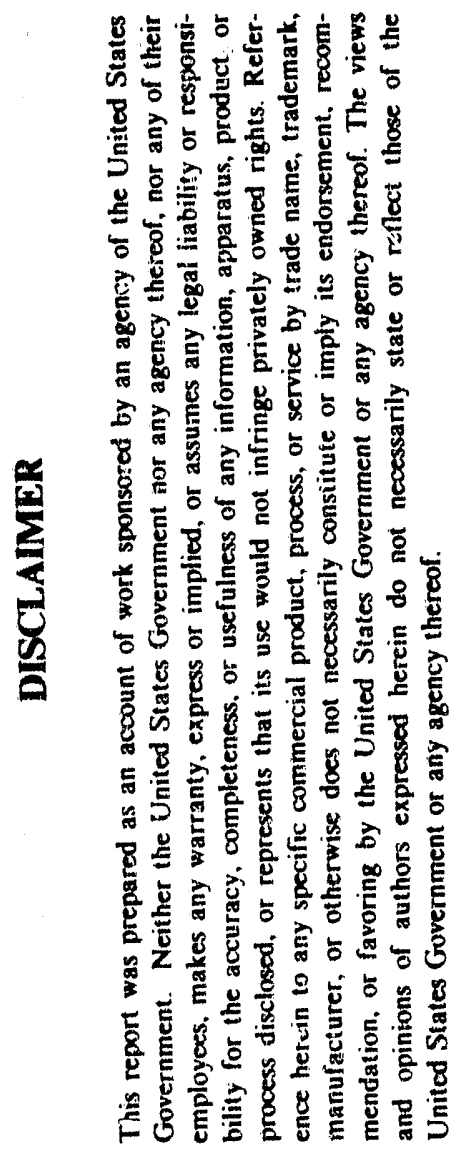

December 1990

National Low-Level Waste

Management Program

Idaho Falls, Idaho 83415

Prepared by EG\&G luaho, Inc.

for the U.S. Departmen: of Energy

Under DOE Contract No. DE-AC07-76ID01570

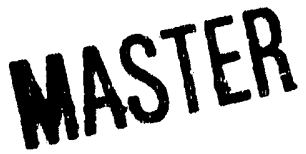




\begin{abstract}
The National Low-Level Waste Management Program has published eleven annual State-by-State assessment reports. These reports provide both national and state-specific disposal data on low-level radioactive wastes. Data in this report are divided into generator category, waste class, volume, and activity. Included in this report are tables showing a distribution of wastes by state for 1989 and a comparison of waste volumes by state for 1985 through 1989: also included is a list of all commercial nuclear power reactors in the United States as of December 31, 1989. In this year's report, a distinction has been made between low-level radioactive waste shipped directly for disposal by generators and that handled by an intermediary.
\end{abstract}

\title{
NOTICE
}

The information presented in this repont is meant to be informative to the reader and provide accurate disposal figures for commercial low-level radioactive waste being shipped for disposal within the United States. Comments and suggestions as to how this document may better address your needs for information on this subject are welcomed. Comments may be sent to the address listed below:

Department of Energy

National Low-Level Waste Management Program

P.O. Box 1625

Idaho Falls, Idaho 83415-3960 


\section{CONTENTS}

ABSTRACT

1. INTRODUCTION $\ldots \ldots \ldots \ldots \ldots \ldots \ldots \ldots \ldots \ldots \ldots \ldots \ldots \ldots \ldots \ldots \ldots \ldots$

2. 1989 REPORT METHOD AND SUMMARY $\ldots \ldots \ldots \ldots \ldots \ldots \ldots \ldots \ldots \ldots$

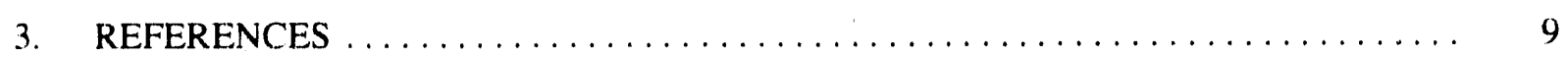

APPENDIX A-1989 LOW-LEVEL WASTE ASSESSMENTS BY COMPACT REGION AND STATE $\ldots \ldots \ldots \ldots \ldots \ldots \ldots \ldots \ldots \ldots \ldots \ldots \ldots \ldots \ldots \ldots \ldots \ldots$

INDEX OF STATES $\ldots \ldots \ldots \ldots \ldots \ldots \ldots \ldots \ldots \ldots \ldots \ldots \ldots \ldots \ldots \ldots \ldots \ldots \ldots \ldots$

APPENDIX B-WASTE VOLUME COMPARISON BY STATE AND COMMERCIAL NUCLEAR POWER REACTORS IN THE UNITED STATES $\ldots \ldots \ldots \ldots \ldots$ B-1

APPENDIX C - STATE RADIATION PROGRAM REPRESENTATIVES $\ldots \ldots \ldots \ldots \ldots \ldots$ 


\section{STATE-BY-STATE ASSESSMENT OF LOW-LEVEL RADIOACTIVE WASTES RECEIVED AT COMMERCIAL DISPOSAL SITES}

\section{INTRODUCTION}

The National Low-Level Waste Management Program has published eleven annual State-by-State assessment reports. These reports provide both National and State-specific disposal data that assist States in the management of low-level radioactive waste (LLW), as specified in the Low-Level Radioactive Waste Policy Act (Public Law 96-573) and the Low-Level Radioactive Waste Policy Amendments Act of 1985 (LLRWPAA, Public Law 99-240).

Over the past eleven years, every State has generated some quantity of commercial LLW. These wastes are generated from a variety of commercial sources such as:

- Government and industrial research

- Generation of electrical power

- Medical diagnostic and therapeutic services

- Various manufacturing processes.

Before publication of the first State-by-State report in 1979, researchers studied the quantities and radioactivity levels of LLW disposed of in the United States. The first report was designed to assist states in extrapolating accurate estimates of disposal rates by separating wastes into the following four broad categories:

- Institutional

- Industrial

- Government

- Utility.

In 1983, the categories were further refined by establishing more specific categories such as:

- Academic

- Medical

- Government

- Industrial

- Utility. 
Commercial disposal sites report the actual volumes disposed of for each of these five categories.

The volume of LLW disposed of at commercial disposal sites exceeded 3.7 million cubic feet in 1980 . This represents the highest reported annual volume of LLW disposed of during the history of the state-by-state assessment documents. From 1981 through 1989, the volume of LLW being disposed of at commercial disposal sites declined. In 1989, the reported volume of LLW received for cisposal at commercial sites was $1,627,813$ cubic feet.

1989 disposed LLW represents a less-than $2 \%$ increase over the 1988 disposed volume. This increase can be attributed to:

- Increase in disposal cost, scheduled by the Low-Level Radioactive Waste Policy Amendments Act of 1985, effective January 1,1990, and

- Cyclic maintenance for selected nuclear power rea.tors.

The scheduled maintenance of power reactors is the dominant factor in the increase in radioactivity for the 1989 LLW disposal. Neither event is expected to have a lasting impact on the downward trend of LLW expected to be disposed of in future years. A list of the $1989 \mathrm{LLW}$ volumes and radioactivity by state is provided in Table 1. Only Puerto Rico, which is treated as a state under the LLRWPAA, reported no LLW shipments in 1989.

The first state-by--state assessment used several data sources. These included U.S. Nuclear Regulatory Commission (NRC) records on nuclear power plant waste shipments, ${ }^{1}$ the U.S. Department of Energy Solid Waste Information Management Systems (SWIMS) ${ }^{2}$ for information on defense wastes shipped to cornmercial sites, and the University of Maryland (at Baltimore) studies on national institutional waste generation. ${ }^{3.4}$ Since its initial publication, this report has been modified and expanded as more detailed state information became available from the disposal sites. Results from generator surveys and feedback from state officials continue to improve understanding of the sources of LLW and the quality of the data.

Data in this report have been quality checked by comparing data from two sources: (1) monthly disposal activity reports supplied by the regulatory agencies of the sited states of Nevada, South Carolina, and Washington (considered as the official sited state data); (2) shipment manifest data obtained from the three commercial LLW disposal sites for LLW received.

In addition to the verification process, a peer review of the draft report was conducted by representatives of the individual states' radiation control programs. A list of state radiation control program representatives is provided for the reader's convenience in Appendix $\mathrm{C}$. 
Table 1. 1989 Waste Distribution by State, as Reported by Disposal Site Operators
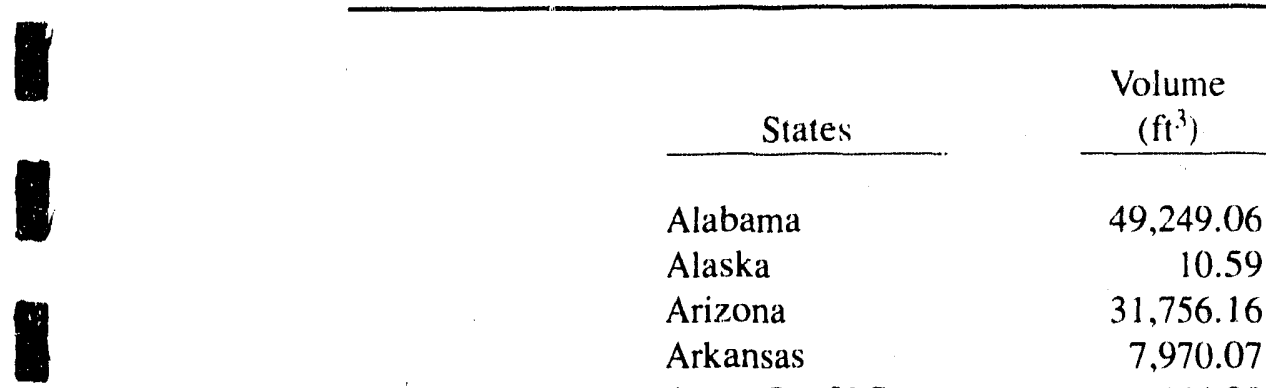

Alabama

Alaska

Arizona

Arkansas

Army Out U.S.

$49,249.06$

10.59

$31,756.16$

$7,970.07$

164.30

California

Colorado

Connecticut

Delaware

District of Columbia

$$
\begin{array}{r}
117,965.93 \\
8,754.29 \\
49,078.29 \\
1,376.41 \\
925.09
\end{array}
$$

Florida

Georgia

Hawaii

Idaho

Illinois

Indiana

lowa

Kansas

Kentucky

Louisiana

Maine

Maryland

Massachusetts

Michigan

Minnesota

Mississippi

Missouri

Montana

Nebraska

Nevada

New Hampshire

New Jersey

New Mexico

New York

North Carolina

$35,282.54$

$44,697.36$

$6,203.82$

137.99

$134,786.89$

$2,150.31$

$6,055.37$

$6,365.39$

$10,047.07$

$21,723.21$

$15,633.40$

$39,995.65$

$56,527.07$

$43,111.39$

$21,954.29$

$10,995.47$

$18,802.64$

107.01

$16,699.15$

307.65

26.70

$53,539.95$

1,369.09

$96,893.38$

$58,421.49$
Activity

(curies)

$1,163.55$

3.90

$1,123.27$

322.16

192.92

$9,949.05$

$1,102.84$

$21,884.63$

2.00

0.69

$2,624.41$

$2,887.45$

6.51

1.70

$147,114.64$

63.13

$16,953.42$

$2,471.22$

21.09

$1,478.13$

273.78

648.32

$57,385.70$

$7,185.79$

$60,952.08$

222.45

793.03

1.06

328.26

789.32

26.92

$317,832.66$

94.54

$101,453.86$

$7,390.08$ 
Table 1. (continued)

\begin{tabular}{|c|c|c|}
\hline States & $\begin{array}{c}\text { Volume } \\
\left(\mathrm{ft}^{3}\right)\end{array}$ & $\begin{array}{l}\text { Activity } \\
\text { (curies) }\end{array}$ \\
\hline North Dakota & 105.20 & 1.84 \\
\hline Ohio & $58,866.18$ & $1,211.40$ \\
\hline Oklahoma & $32,126.93$ & 4.25 \\
\hline Oregon & $75,907.48$ & 465.75 \\
\hline Pennsylvania & $129,484.73$ & 78.347 .84 \\
\hline Rhode Island & $1,419.31$ & 22.70 \\
\hline South Carolina & $96,695.37$ & $2,430.99$ \\
\hline South Dakota & 0.60 & 0.50 \\
\hline Tennessee & $118,957.13$ & $3,043.58$ \\
\hline Texas & $22,157.48$ & $11,357.03$ \\
\hline Utah & $6,299.31$ & 7.88 \\
\hline Vermont & 173.20 & 2.15 \\
\hline Virginia & $82,834.30$ & $3,053.52$ \\
\hline Washington & $26,408.54$ & $1,105.04$ \\
\hline West Virginia & 411.14 & 23.48 \\
\hline Wisconsin & $6,880.93$ & $1,074.88$ \\
\hline Wyoming & 0.50 & 0.19 \\
\hline Total & $1,627,812.80$ & $866,897.56$ \\
\hline
\end{tabular}




\section{1989 REPORT METHOD AND SUMMARY}

The data for the 1989 State-by-State report were developed from the Department of Energy's Manifest Information System, with data originating from the LLW shipment manifests recorded at the three commercial LLW disposal sites located at Barnwell, South Carolina; Beatty, Nevada; and Richland, Washington. ${ }^{5.6}$ This information is presented in Appendix A.

Reports generated in this series from 1979 through 1987 have attributed waste that was processed or consolidated into larger shipments at intermediate waste management facilities to the states in which those facilities were located. The report generated for 1988, and subsequent years, attributes waste volume, activity, and generator type by source to the original state in which it was generated, even where brokered shipments included waste originally generated in more than one state. This change in reporting method was made possible because of changes in the manifesting requirements for brokered shipments at the sited states. The waste volumes for states such as Tennessee, where a significant volume of waste is processed through several large waste management facilities, were the most notably affected.

Two classifications of waste shipments are identified in this year's report.

- Non-Direct

- Shipments from the generator to the disposal facility through an intermediary

- $\quad$ Direct

- Shipments that did not involve an intermediary.

In 1989 approximately $27 \%$ of all waste shipped for disposal was categorized as Non-Direct.

The data are arranged to allow the reader to locate information by area of interest, ranging from total commercial LLW disposed of within the United States to LLW disposed of by a specific State.

Information presented in this report covers the distribution of LLW by:

- Volume

- Activity

- Waste classification

- Waste generator type

- Disposal site

- Waste that has been subject to intermediate handling. 
Waste generator types include:

- Utility

- Academic

- Medical

- Government

- Industrial.

The utility cat gory includes commercial nuclear power reactors. The academic category includes university hospitals and university medical and nonmedical research facilities. Medical generators include hospitals and clinics, research facilities, and private medical offices. The industrial category includes private entities such as research and development companies, manufacturers, nondestructive testing, mining, fuel fabrication facilities, and radiopharmaceutical manufacturers. The government category includes State and Federal agencies.

Appendix B contains a comparison of LLW disposal volumes by state for the most recent five-year period, and a complete list of the commercial nuclear power reactors located in the United States as of December 31, 1989.

A review of the data found in this report will show that, in 1989, commercial LLW disposal facilities received a total volume of $1,627,813$ cubic feet of waste, containing an activity of 866,898 curies. Waste distribution by disposal site is presented in Table 2; Tables 3 and 4 provide typical radionuclide and waste forms associated with commercial LLW. 
Table 2. Distribution of Low-Level Radioactive Waste Received at Disposal Sites in 1989

\begin{tabular}{|c|c|c|c|c|}
\hline Site & $\begin{array}{c}\begin{array}{c}\text { Volume } \\
\left(\mathrm{ft}^{3}\right)\end{array} \\
\end{array}$ & $\begin{array}{c}\text { Percent } \\
\text { of } \\
\text { Total } \\
\end{array}$ & $\begin{array}{l}\text { Activity } \\
\text { (curies) }\end{array}$ & $\begin{array}{c}\text { Percent } \\
\text { of } \\
\text { Total } \\
\end{array}$ \\
\hline Barnwell & $1,103,300$ & 68 & 725,164 & 84 \\
\hline Beatty & 116,222 & 7 & 42,678 & 5 \\
\hline Richland & 408,291 & 25 & 99,056 & 11 \\
\hline Total & $1,627,813$ & 100 & 866,898 & 100 \\
\hline
\end{tabular}

Table 3. Most Frequently Reported Low-Level Radioactive Waste Radionuclides Received at Disposal Sites in 1989

\begin{tabular}{|c|c|c|c|c|}
\hline Reactors & Academic & Medical & Industrial & Government \\
\hline${ }^{61)} \mathrm{Co}$ & ${ }^{12.5} \mathrm{I}$ & ${ }^{51} \mathrm{Cr}$ & NatTh & ${ }^{61)} \mathrm{Co}$ \\
\hline${ }^{14} \mathrm{C}$ & ${ }^{3} \mathrm{H}$ & ${ }^{3} \mathrm{H}$ & ${ }^{\mathrm{Nat}} \mathrm{U}$ & ${ }^{55} \mathrm{Fe}$ \\
\hline${ }^{55} \mathrm{Fe}$ & ${ }^{35} \mathrm{~S}$ & $125 \mathrm{I}$ & ${ }^{238} \mathrm{U}$ & ${ }^{54} \mathrm{Mn}$ \\
\hline${ }^{3} \mathrm{H}$ & ${ }^{14} \mathrm{C}$ & ${ }^{35} \mathrm{~S}$ & ${ }^{14} \mathrm{C}$ & ${ }^{63} \mathrm{Ni}$ \\
\hline${ }^{137} \mathrm{Cs}$ & ${ }^{51} \mathrm{Cr}$ & ${ }^{14} \mathrm{C}$ & ${ }^{3} \mathrm{H}$ & ${ }^{58} \mathrm{Co}$ \\
\hline${ }^{54} \mathrm{Mn}$ & ${ }^{32} \mathrm{P}$ & ${ }^{57} \mathrm{Co}$ & ${ }^{137} \mathrm{Cs}$ & ${ }^{14} \mathrm{C}$ \\
\hline${ }^{58} \mathrm{Co}$ & ${ }^{45} \mathrm{Ca}$ & ${ }^{59} \mathrm{Fe}$ & ${ }^{6(1)} \mathrm{Co}$ & $c$ \\
\hline${ }^{99} \mathrm{Tc}$ & & ${ }^{32} \mathrm{P}$ & ${ }^{241} \mathrm{Pu}$ & \\
\hline${ }^{63} \mathrm{Ni}$ & & $238 \mathrm{U}$ & ${ }^{226} \mathrm{Ra}$ & \\
\hline $129 \mathrm{~J}$ & & & & \\
\hline
\end{tabular}


Table 4. Typical Waste Forms, by Generator Categories ${ }^{7}$

\section{Utilities}

Spent resins

Evaporator bottoms and concentrated waste

Filter sludges

Dry compressible waste

Irradiated components

Contaminated plant hardware

\section{Academic}

Compacted trash or solids

Institutional laboratory or biological waste

Absorbed liquids

Animal carcasses

\section{Medical}

Compacted trash or solids

Institutional laboratory or biological waste

Absorbed liquids

Sealed sources

\section{Industrial}

Depleted uranium

Compacted trash or solids

Contaminated plant hardware

Absorbed liquids

Sealed sources

\section{Government}

Compacted trash or solids

Contaminated plant hardware

Absorbed liquids 


\section{REFERENCES}

1. U.S. Nuclear Regulatory Commission, "Measuring, Evaluating, and Reporting Radioactivity in Solid Wastes and Releases of Radioactive Materials in Liquid and Gaseous Effluents from Light Water Cooled Nuclear Power Plants," Regulatory Guide 1.21, Revision 1, June 1974.

2. U.S. Department of Energy, Washington, D.C., "Solid Waste Information Management System (SWIMS)-Actual Solid Waste Generated for Fiscal Year 1979:" February 22, 1980.

3. R. L. Anderson, L. R. Cooley, T. J. Beck, C. S. Strauss, Institutional Radioactive Waste, NUREG/ CR-0028, Radiation Safety Office, University of Maryland at Baltimore, March 1978.

4. T. J. Beck, L. R. Cooley, M. R. McCampbell, Institutional Radioactive Wastes-1977, Radiation Safety Office, University of Maryland at Baltimore (draft), June 1979.

5. Data obtained from Chem-Nuclear Systems, Inc., for the Barnwell, South Carolina low-level radioactive waste disposal site, as defined by subcontract number C87-131476.

6. Data obtained from U.S. Ecology, Inc. operated low-level radioactive waste disposal sites located in Beatty, Nevada and Richland, Washington, from Utility Data Institute, as defined by subcontract number C87--131477. Utility Data Institute is the exclusive licensee and marketing agent for the American Ecology Time Sharing Service.

7. Conference of Radiation Control Program Directors, Inc., Low-Level Radioactive Waste Management Survey Summary, 1984. 


\section{APPENDIX A}

1989 LOW-LEVEL RADIOACTIVE WASTE ASSESSMENTS BY

COMPACT REGICIN AND STATE

The volume and activity totals on the following pages may not sum exactly due to computer-generated rounding.

$$
\mathrm{A}-\mathrm{i} / \mathrm{ii}
$$




\section{INDEX OF STATES}

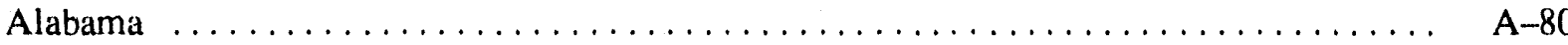

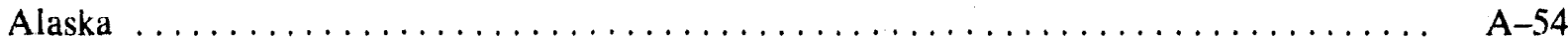

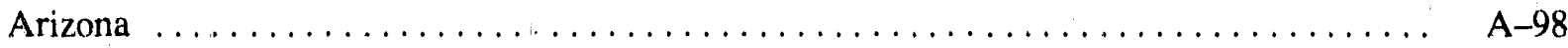

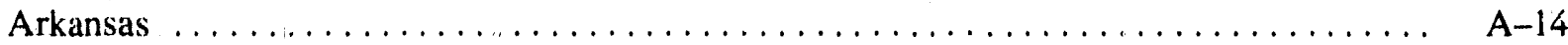

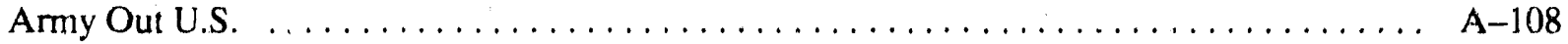

California ..................................... A-190

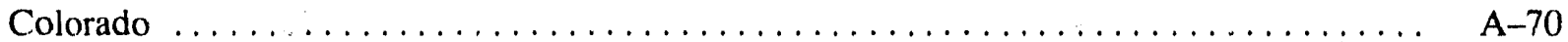

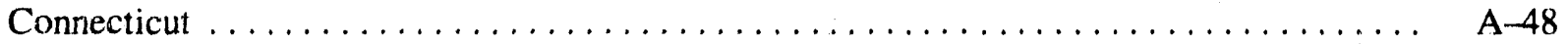

Deiaware ..................................... A

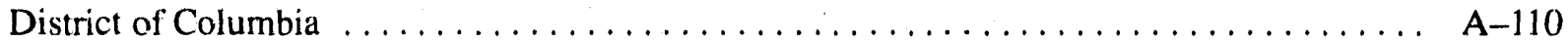

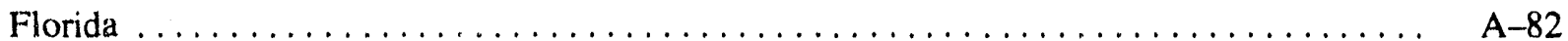

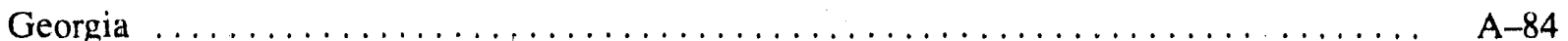

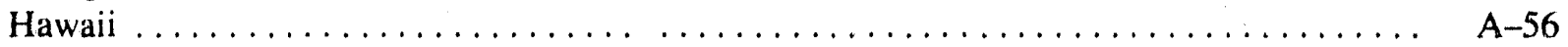

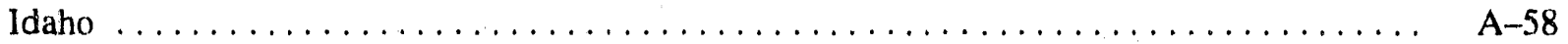

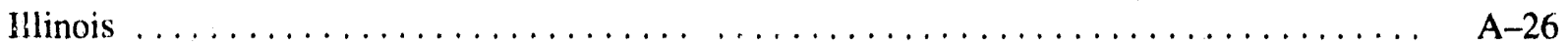

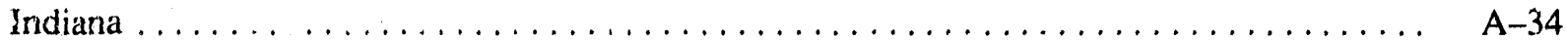

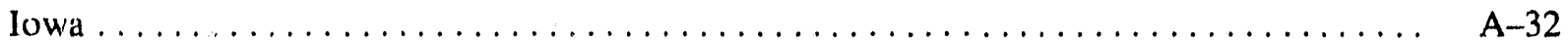

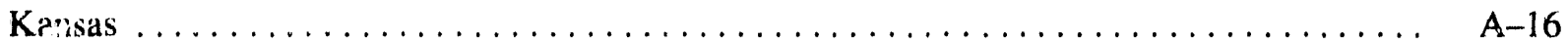

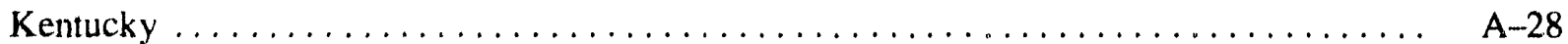

Louisiana ...................................... A-19

Maine ........................................ A-112

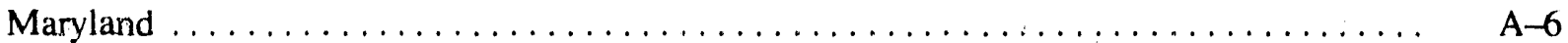

Massachusetts .................................... A-114

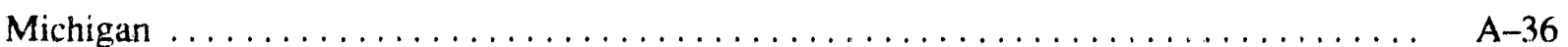

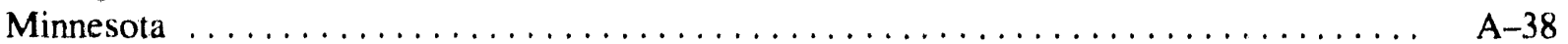

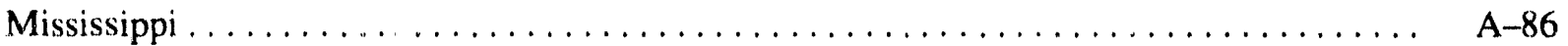

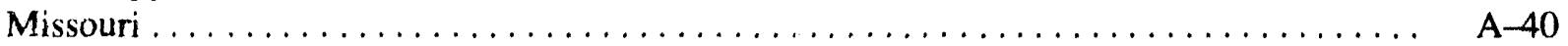

Montana ...................................... A-60

Nebraska ....................................... A-20

Nevada ......................................... A-72

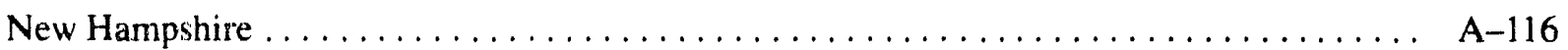

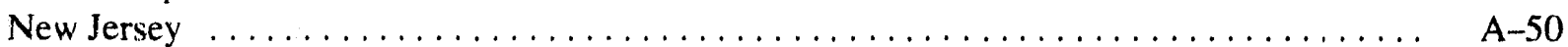

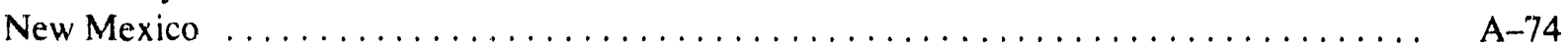

New York ..................................... A-118

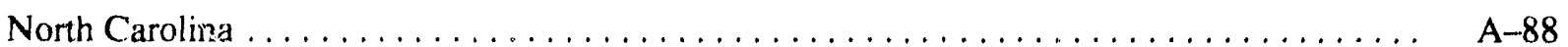

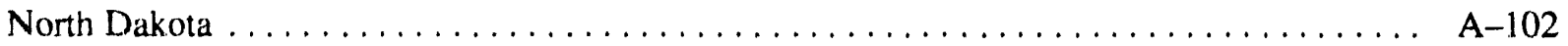

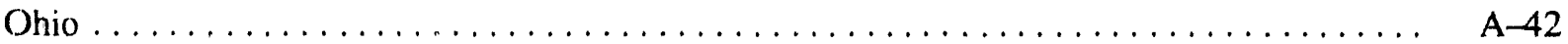

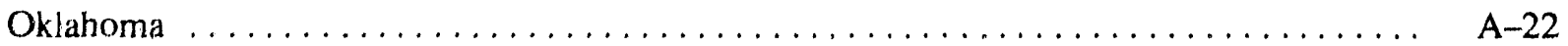

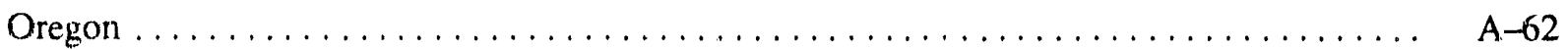

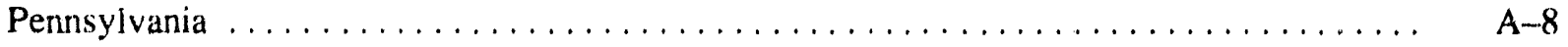

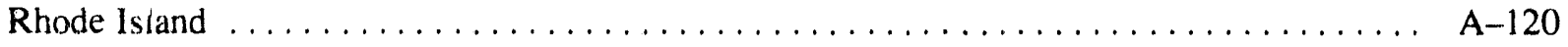

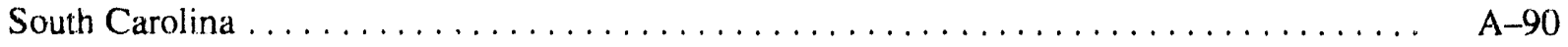

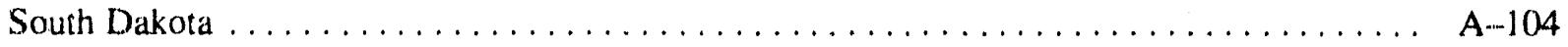

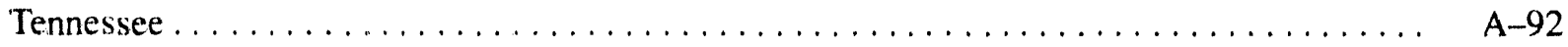

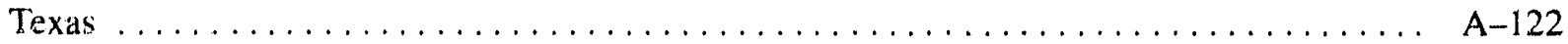




\section{INDEX OF STATES}

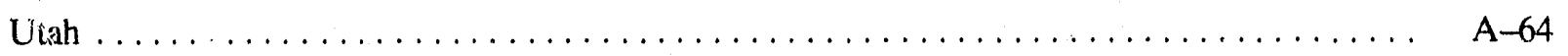

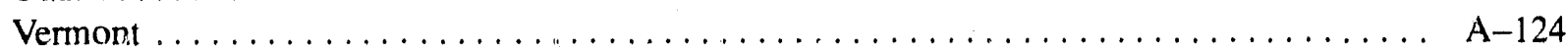

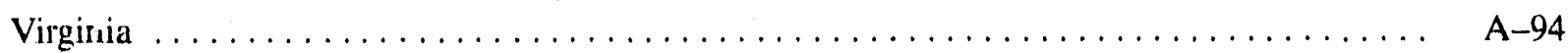

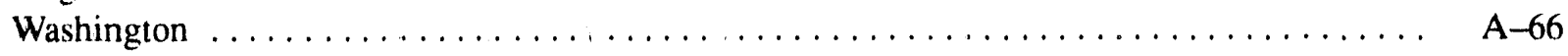

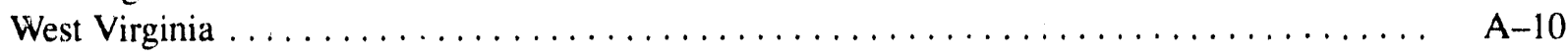

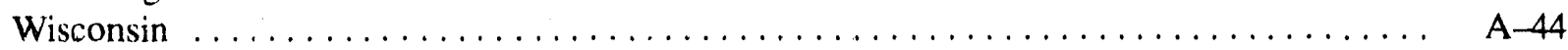

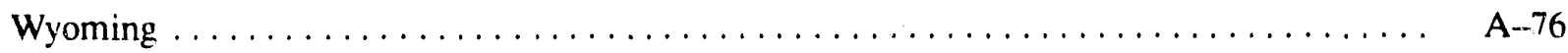




\section{UNITED STATES \\ Low-Level Radioactive Wastes Received at Commercial Disposal Sites ${ }^{a}$}

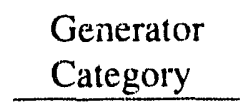

Academic

Government

Industrial

Medical

Utility

Total
Volume

$\left(\mathrm{ft}^{3}\right)$

$06,101.42$

$114,090.40$

$565,567.74$

$34,730.20$

$847,323.04$

$1,627,812.80$
Activity

(curies)

$1,946.44$

$12,556.70$

$127,019.54$

149.32

$725,225.56$

$866,897.56$

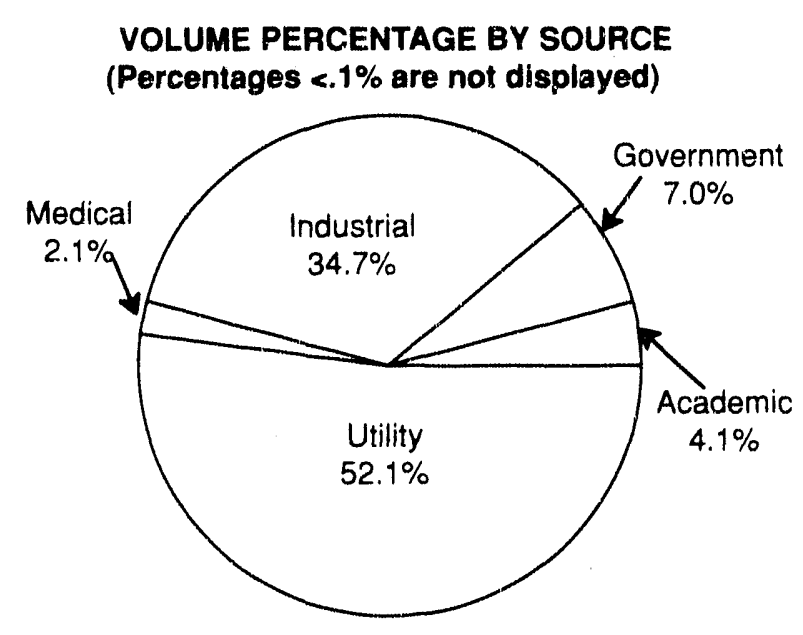

ACTIVITY PERCENTAGE BY SOURCE
(Percentages $<.1 \%$ are not displayed)

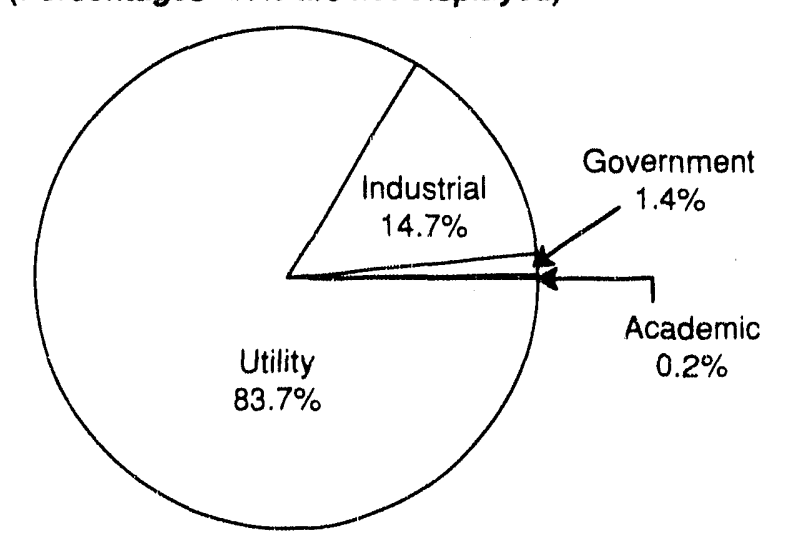

a. Data in this, and all succeeding pages in Appendix A, were provided by the Department of Energy's Manifest Information System. Data originated with Chem Nuclear Systems, Inc., and Utility Data Inst., Inc., from References (5) and (6) of the main text. 


\section{UNITED STATES \\ Low-Level Radioactive Wastes Received at Commercial Disposal Sites}

\begin{tabular}{c}
$\begin{array}{c}\text { Disposal } \\
\text { Site }\end{array}$ \\
\hline
\end{tabular}

Barnwell

Beatty

Richland

Total

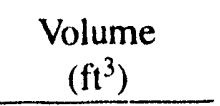

$1,103,299.56$

$116,222.12$

$408,291.12$

$1,627,812.80$
Activity

(curies)

$725,163.57$

$42,678.37$

$99,055.62$

$866,897.56$
VOLUME PERCENTAGE BY DISPOSAL SITE (Percentages <.1\% are not displayed)

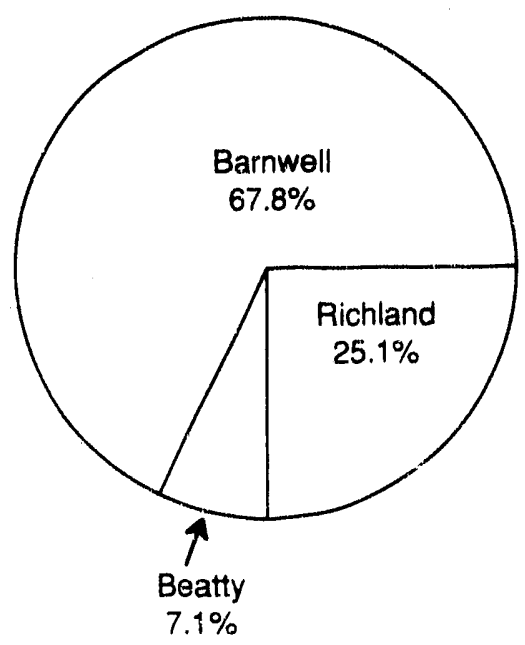

ACTIVITY PERCENTAGE BY DISPOSAL SITE (Percentages <.1\% are not displayed)

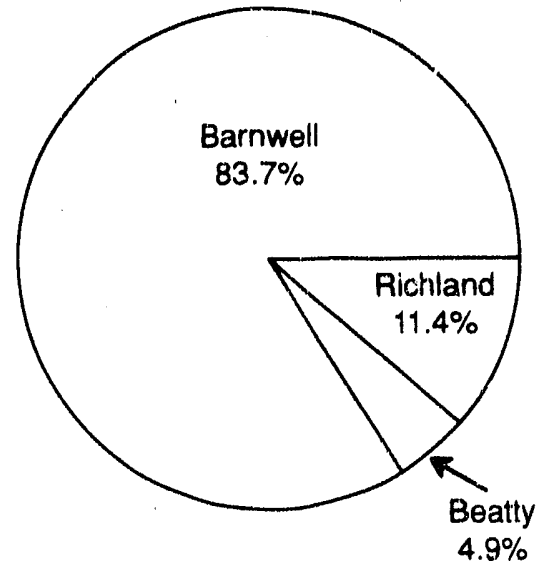

Volume Percentage by Waste Class for Direct Shipments und Total Non-Direct Volume Percentage (Percentages <.1\% are not displayed)

\section{Waste Class}

A

B

C

Total

Total Activity (curies)
Volume

$$
\left(\mathrm{ft}^{3}\right)
$$

$1,575,378.96$

$35,942.46$

$16,491.38$

$1,627,812.80$

$866,898.76$

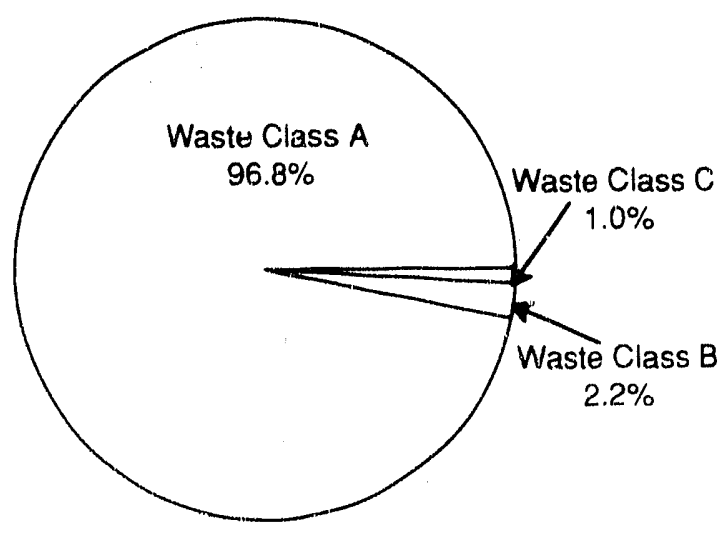




\section{APPALACHIAN COMPACT \\ Low-Level Radioactive Wastes Received at Commercial Disposal Sites}

\begin{tabular}{lrr}
\multicolumn{1}{c}{ States } & \multicolumn{1}{c}{$\begin{array}{c}\text { Volume } \\
\left(\mathrm{ft}^{3}\right)\end{array}$} & \multicolumn{1}{c}{$\begin{array}{c}\text { Activity } \\
\text { (curies) }\end{array}$} \\
Delaware & $1,376.41$ & 2.00 \\
Maryland & $39,995.65$ & $\begin{array}{r}648.32 \\
\text { Pennsylvania }\end{array}$ \\
West Virginia & $129,484.73$ & $78,347.84$ \\
& 411.14 & 23.48 \\
Total & $171,267.93$ & $79,021.63$
\end{tabular}

VOLUME PERCENTAGE BY STATE

(Percentages <.1\% are not displayed)

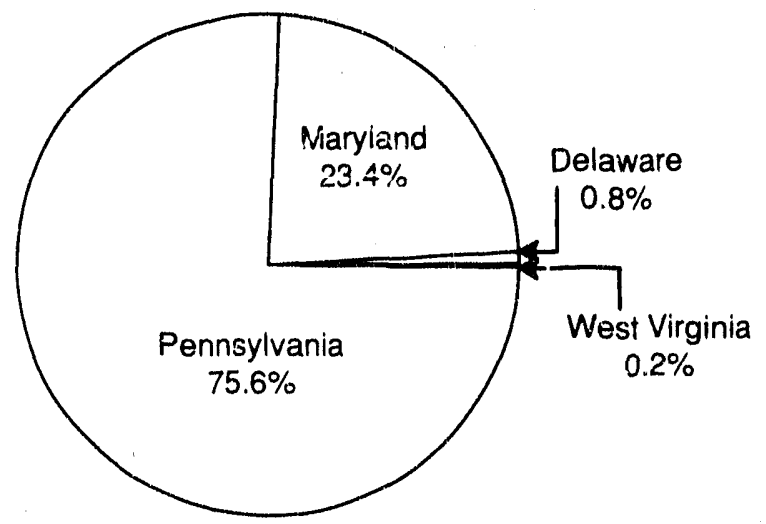

VOLUME PERCENTAGE BY CATEGORY

(Percentages <. $1 \%$ are not displayed)

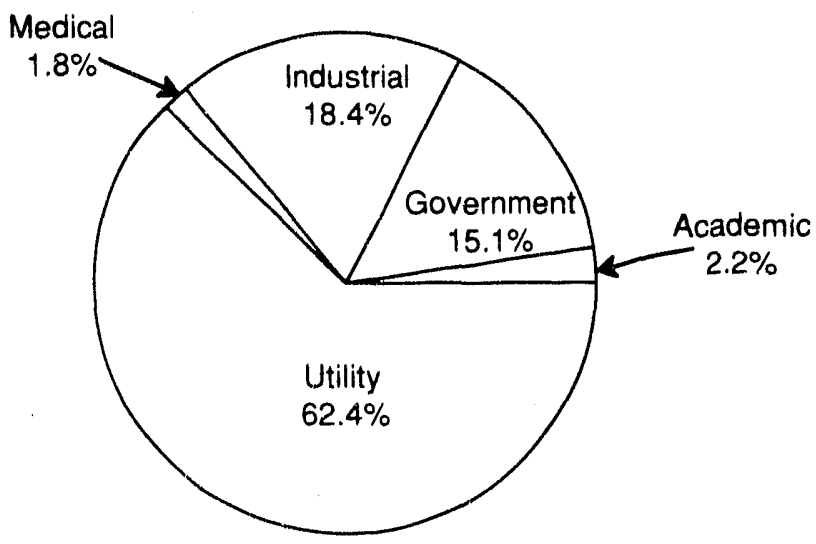




\section{DELAWARE \\ Low-Level Radioactive Wastes Received at Commercial Disposal Sites}

\begin{tabular}{l} 
Generator \\
Category \\
\hline
\end{tabular}

Academic

Industrial

Total

\begin{tabular}{c} 
Volume \\
$\left(\mathrm{ft}^{3}\right)$ \\
\hline
\end{tabular}

27.67

$1,348.74$

$1,376.41$
Activity

(curies)

0.03

1.97

2.00
VOLUME PERCENTAGE BY SOURCE

(Percentages <. $1 \%$ are not displayed)

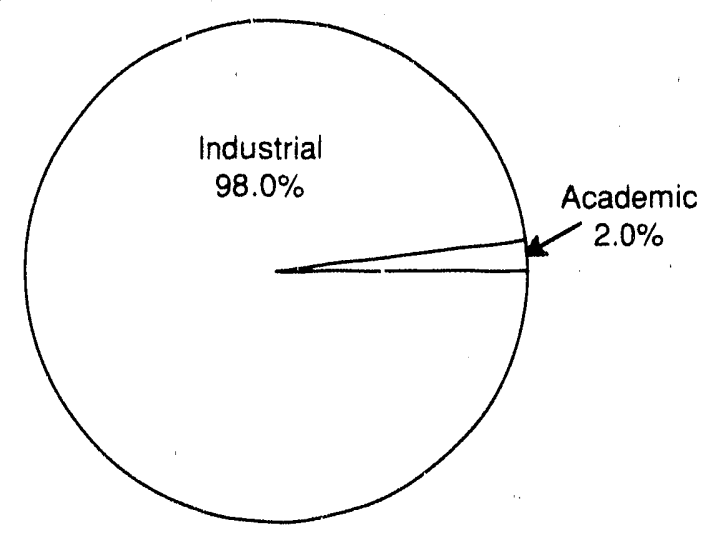

ACTIVITY PERCENTAGE BY SOURCE

(Percentages $<.1 \%$ are not displayed)

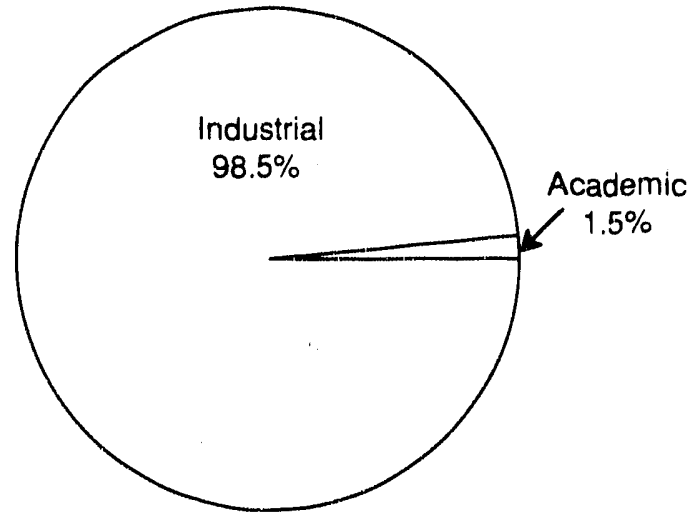




\section{DELAWARE \\ Low-Level Radioactive Wastes Received at Commercial Disposal Sites}

\begin{tabular}{lrr}
\multicolumn{1}{c}{$\begin{array}{c}\text { Disposal } \\
\text { Site }\end{array}$} & $\begin{array}{c}\text { Volume } \\
\left(\mathrm{ft}^{3}\right)\end{array}$ & $\begin{array}{r}\text { Activity } \\
\text { (curies) }\end{array}$ \\
Barnwell & 168.64 & 0.11 \\
Beatty & 35.34 & 1.49 \\
Richland & $1,172.43$ & 0.40 \\
Total & $1,376.41$ & 2.00
\end{tabular}

VOLUME PERCENTAGE BY DISPOSAL SITE (Percentages <. $1 \%$ are not displayed)

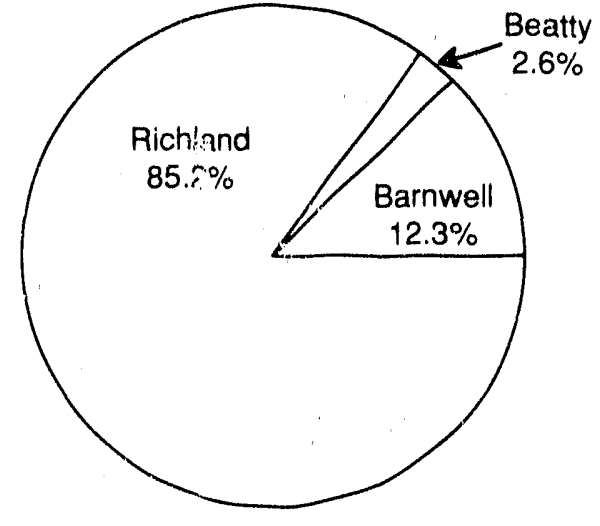

ACTIVITY PERCENTAGE BY DISPOSAL SITE (Percentages $<.1 \%$ are not displayed)

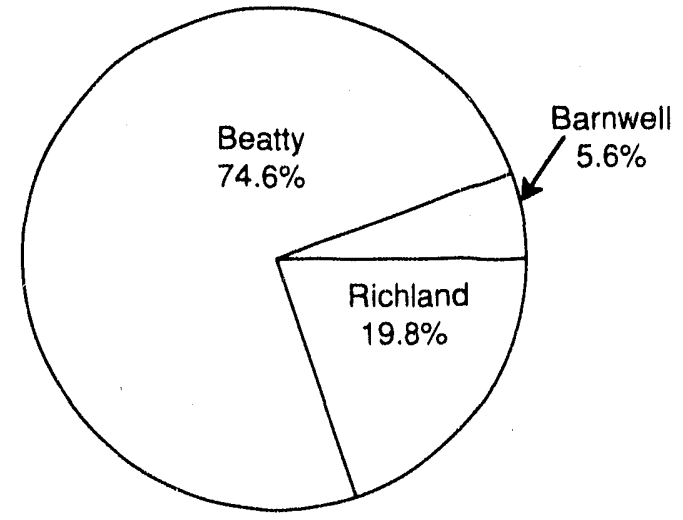

Volume Percentage by Waste Class

for Direct Shipments and Total

Non-Direct Volume Percentage

(Percentages <. 1\% are not displayed)

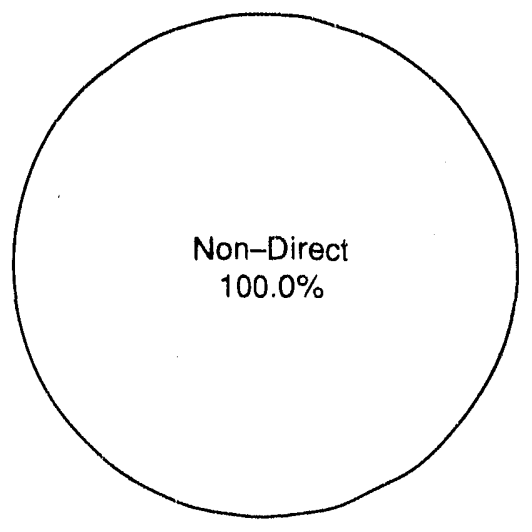




\section{MARYLAND \\ Low-Level Radioactive Wastes Received at Commercial Disposal Sites}

\author{
Generator \\ Category \\ Academic \\ Government \\ Industrial \\ Medical \\ Utility
}

Total

Volume
$\left(\mathrm{ft}^{3}\right)$

852.55

$21,706.59$

$9,346.30$

683.71

$7,406.50$

$39,995.65$
Activity

(curies)

\subsection{5}

161.56

70.60

0.15

413.86

648.32
VOLUME PERCENTAGE BY SOURCE

(Percentages <.1\% are not displayed)

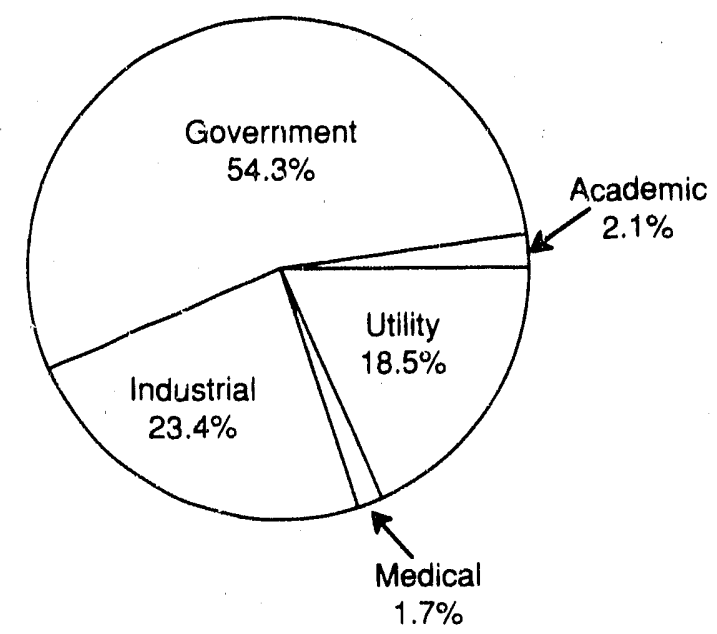

ACTIVITY PERCENTA(SE BY SOURCE

(Percentages <. $1 \%$ are not displayed)

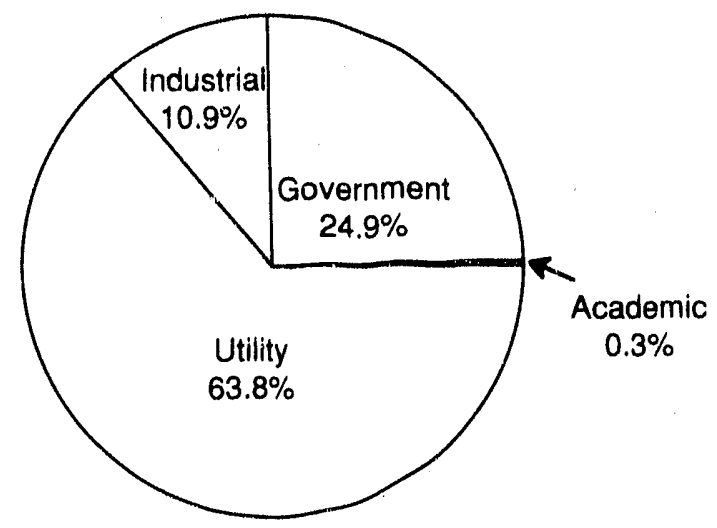




\section{MARYLAND \\ Low-Level Radioactive Wastes Received at Commercial Disposal Sites}

\begin{tabular}{c} 
Disposal \\
Site \\
\hline
\end{tabular}

Barnwell

Beatty

Richland

Total

Volume
$\left(\mathrm{ft}^{3}\right)$

$34,312.30$

$2,589.66$

$3,093.69$

$39,995.65$
Activity (curies)

601.37

39.29

7.66

648.32
VOLUME PERCENTAGE BY DISPOSAL SITE

(Percentages <. $1 \%$ are not displayed)

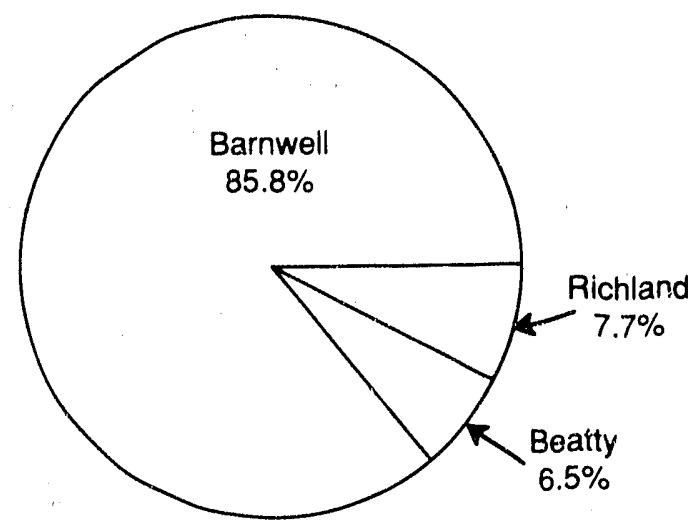

Direct Shipment

Waste Class

A

B

C

Total

Non-Direct Shipments

Grand Total

Total Direct Shipment

Activity (curies)

Total Non-Direct Shipment

Activity (curies)

Grand Total (curies) Volume $\left(\mathrm{ft}^{3}\right)$

$23,883.50$

411.60

126.00

$24,421.10$

$15,574.55$

$39,995.65$

429.87

218.45

648.32

\section{ACTIVITY PERCENTAGE BY DISPOSAL SITE}

(Percentages <. $1 \%$ are not displayed)

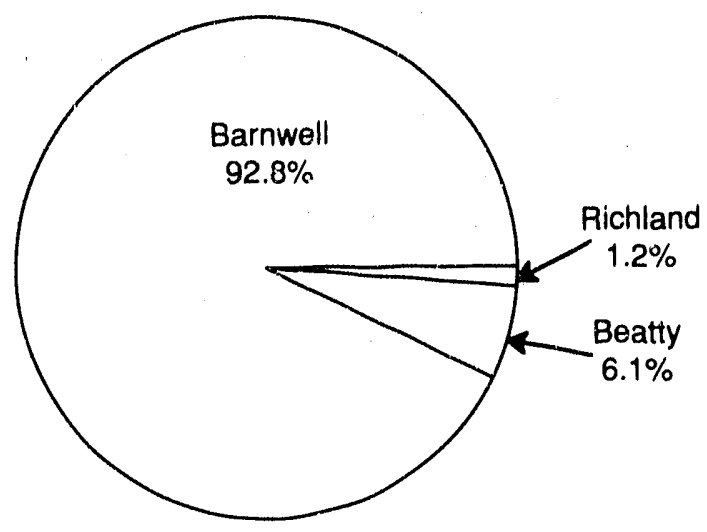

Volume Percentage by Waste Class for Direct Shipments and Total Non-Direct Volume Percentage (Percentages <. $1 \%$ are not displayed)

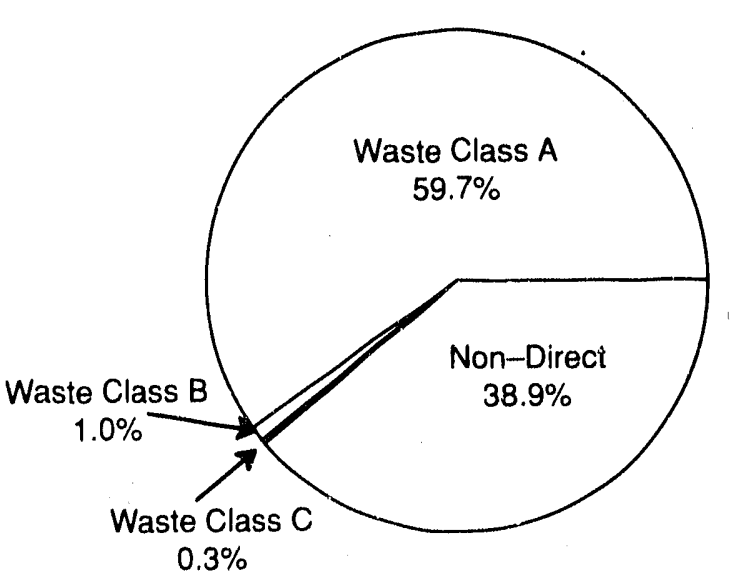




\section{PENNSYLVANIA \\ Low-Level Radioactive Wastes Received at Commercial Disposal Sites}

\begin{tabular}{l} 
Generator \\
Category \\
\hline
\end{tabular}

Acadenic

Government

Industrial

Medical

Utility

Total

$\begin{array}{r}\begin{array}{c}\text { Volume } \\ \left(\mathrm{ft}^{3}\right)\end{array} \\ \hline 2,930.40 \\ 4,138.10 \\ 20,867.80 \\ 2,093.68 \\ 99,454.75 \\ \hline\end{array}$

$129,484.73$
Activity

(curies)

$5,135.68$

$21,073.51$

4.49

$52,120.54$

$78,347.84$
VOLUME PERCENTAGE BY SOURCE

(Percentages <. $1 \%$ are not displayed)

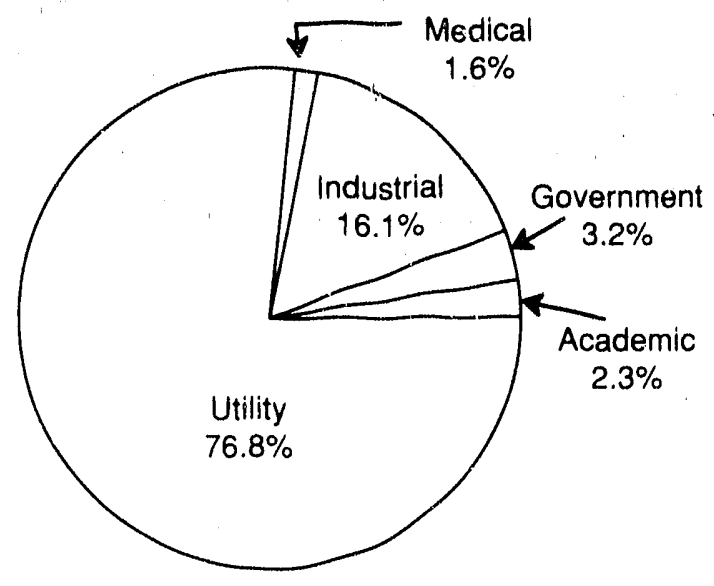

ACTIVITY PERCENTAGE BY SOURCE

(Percentages <. $1 \%$ are not displayed)

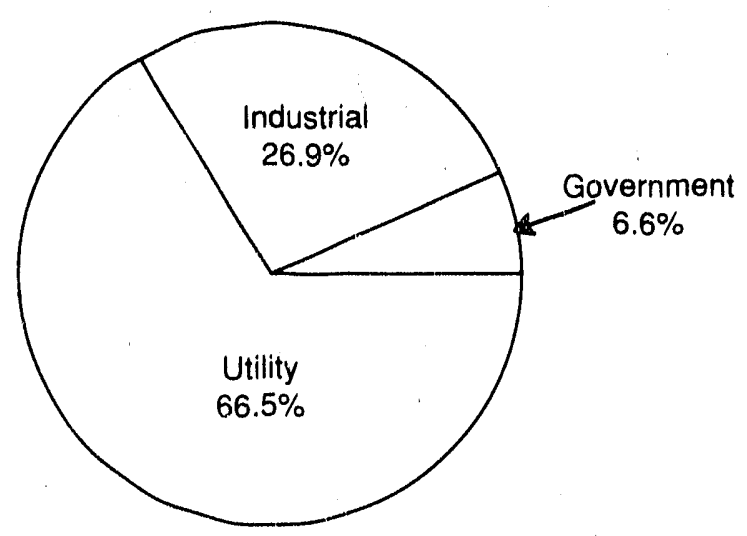




\section{PENNSYLVANIA \\ Low-Level Radioactive Wastes Received at Commercial Disposal Sites}

Disposal
Site

Banwell

Beatty

Richland

Total

\begin{tabular}{c}
$\begin{array}{c}\text { Volurne } \\
\left(\mathrm{ft}^{3}\right)\end{array}$ \\
\hline
\end{tabular}

$106,373.78$

$3,250.59$

$19,860.36$

$129,484.73$
Activity

(curies)

$48,334.87$

$21,019.00$

$8,993.96$

$78,347.84$
VOLUME PERCENTAGE BY DISPOSAL SITE (Percentages $<.1 \%$ are not displayed)

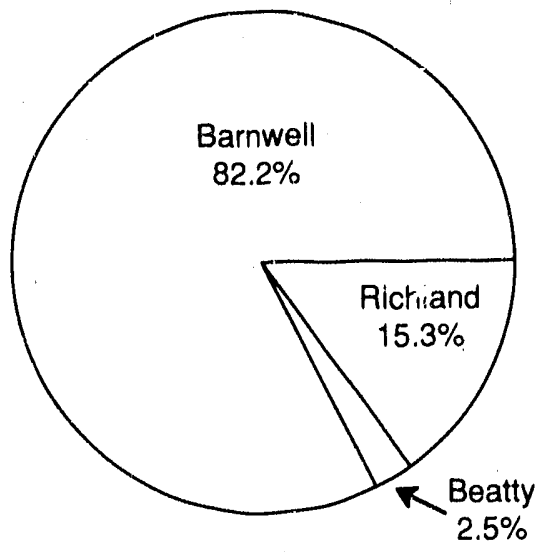

ACTIVITY PERCENTAGE BY DISPOSAL SITE (Percentages <. $1 \%$ are not displayed)

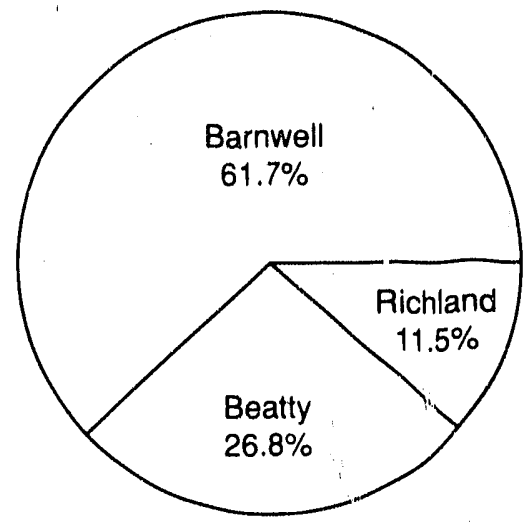

Volume Percentage by Waste Class for Direct Shipments and Total Non-Direct Volume Percentage (Percentages <. $1 \%$ are not displayed)
Direct Shipment

Waste Class

A
B
C

Total

Non-Direct Shipments

Grand Total

Total Direct Shipment

Activity (curies)

Total Non-Direct Shipment

Activity (curies)

Grand Total (curies)
Volume

$\left(\mathrm{ft}^{3}\right)$

$$
\begin{array}{r}
53,568.65 \\
3,267.70 \\
1,527.70 \\
\hline 58,364.05 \\
71,120.68 \\
\hline 129,484.73
\end{array}
$$

$52,028.53$

$\underline{26,319.30}$

$78,347.84$

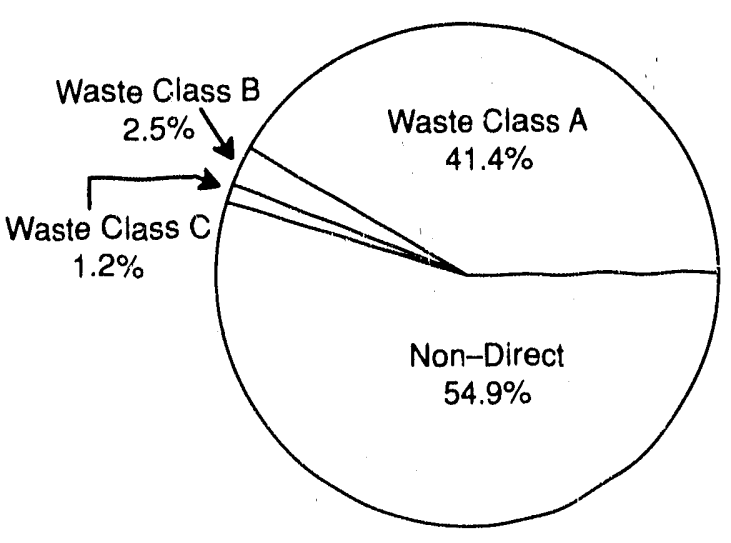




\section{WEST VIRGINIA \\ Low-Level Radioactive Wastes Received at Commercial Disposal Sites}

\begin{tabular}{l} 
Generator \\
Category \\
\hline
\end{tabular}

Academic

Government

Industrial

Medical

Total

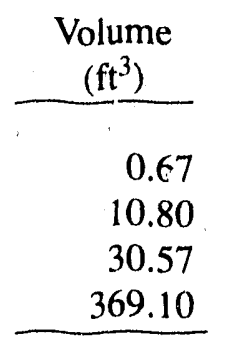

411.14
Activity

(curies)

$<1$

22.10

1.37

23.48

VOLUME PERCENTAGE BY SOURCE

(Percentages <. $1 \%$ are not displayed)

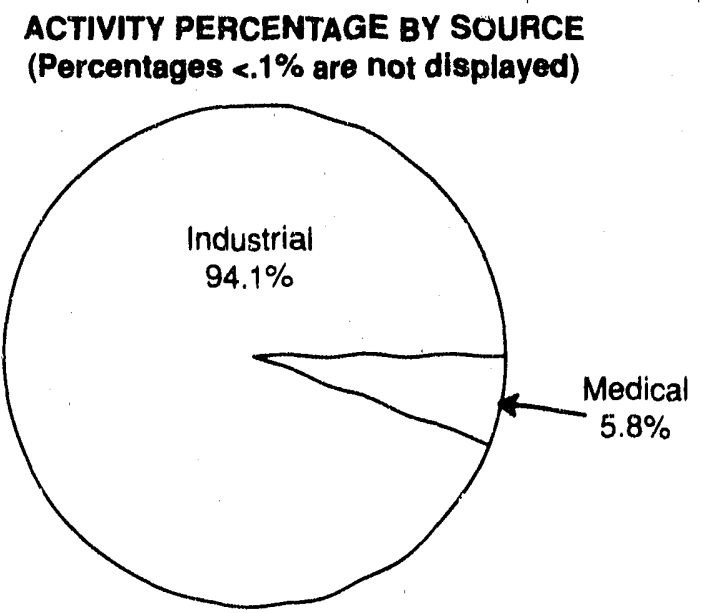




\title{
WEST VIRGINIA \\ Low-Level Radioactive Wastes Received at Commercial Disposal Sites
}

\begin{tabular}{c} 
Disposal \\
Site \\
\hline
\end{tabular}

Beatty

Richland

Total

\begin{tabular}{c}
$\begin{array}{c}\text { Volume } \\
\left(\mathrm{ft}^{3}\right)^{3}\end{array}$ \\
\hline
\end{tabular}

44.04

367.10

411.14
Activity

(curies)

22.22

1.26

23.48
VOLUME PERCENTAGE BY DISPOSAL SITE

(Percentages <. $1 \%$ are not displayed)

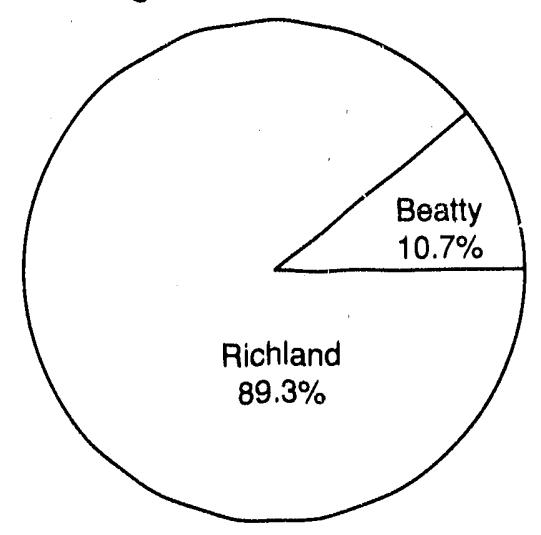

ACTIVITY PERCENTAGE BY DISPOSAL SITE (Percentages <.1\% are not displayed)

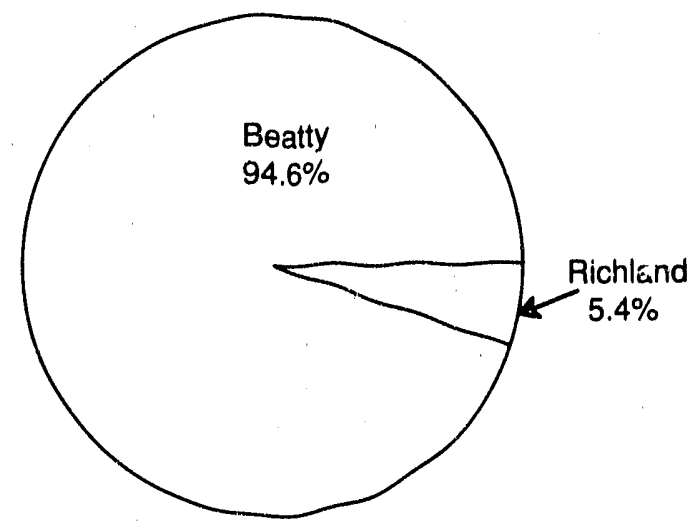

\begin{abstract}
Volume Percentage by Waste Class for Direct Shipments and Total Non-Direct Volume Perceritage (Percentages $<.1 \%$ are not displayed)
\end{abstract}

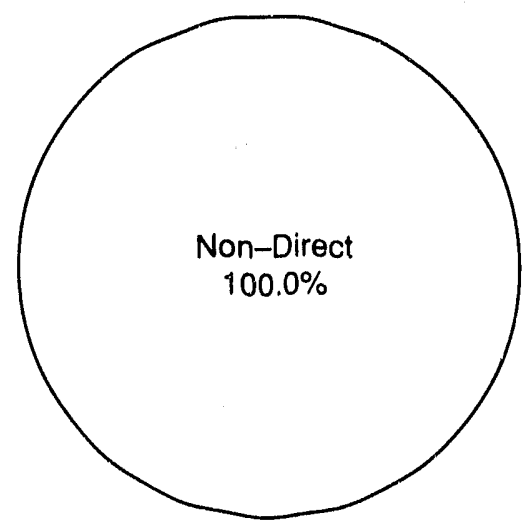

$$
\mathrm{A}-1 / 12
$$




\section{CENTRAL COMPACT \\ Low-Level Radioactive Wastes Received at Commercial Disposal Sites}

\begin{tabular}{lrr}
\multicolumn{1}{c}{ States } & \multicolumn{1}{c}{$\begin{array}{c}\text { Volume } \\
\left(\mathrm{ft}^{3}\right)\end{array}$} & \multicolumn{1}{c}{$\begin{array}{c}\text { Activity } \\
(\text { curies })\end{array}$} \\
Arkansas & $7,970.07$ & 322.16 \\
Kansas & $6,365.39$ & $2,471.22$ \\
Louisiana & $21,723.21$ & $1,478.13$ \\
Nebraska & $16,699.15$ & 328.26 \\
Oklahoma & $32,126.93$ & 4.25 \\
Total & $84,884.75$ & $4,604.02$
\end{tabular}

VOLUME PERCENTAGE BY STATE

(Percentages <. $1 \%$ are not displayed)

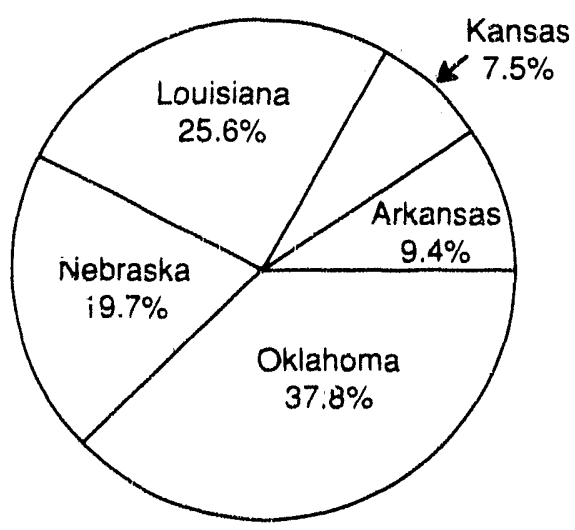

VOLUME PERCENTAGE BY CATEGORY

(Percentages <.1\% are not displayed)

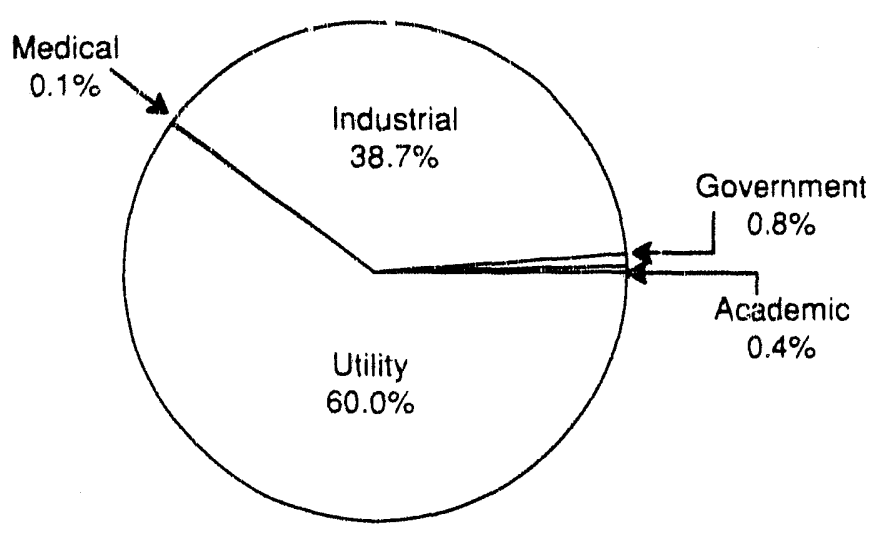




\section{ARKANSAS \\ Low-Level Radioactive Wastes Received at Commercial Disposal Sites}

\begin{tabular}{lrr}
$\begin{array}{l}\text { Generator } \\
\text { Category }\end{array}$ & \multicolumn{1}{c}{$\begin{array}{c}\text { Volume } \\
\left(\mathrm{ft}^{3}\right)\end{array}$} & \multicolumn{1}{c}{$\begin{array}{c}\text { Activity } \\
(\text { curies })\end{array}$} \\
Academic & 56.23 & 0.31 \\
Government & 1.10 & 15.65 \\
Industrial & 26.25 & 10.23 \\
Medical & 54.24 & 0.56 \\
Utility & $7,832.25$ & 295.40 \\
\hline & & 322.16
\end{tabular}

VOLUME PERCENTAGE BY SOURCE

(Percentages <. $1 \%$ are not displayed)

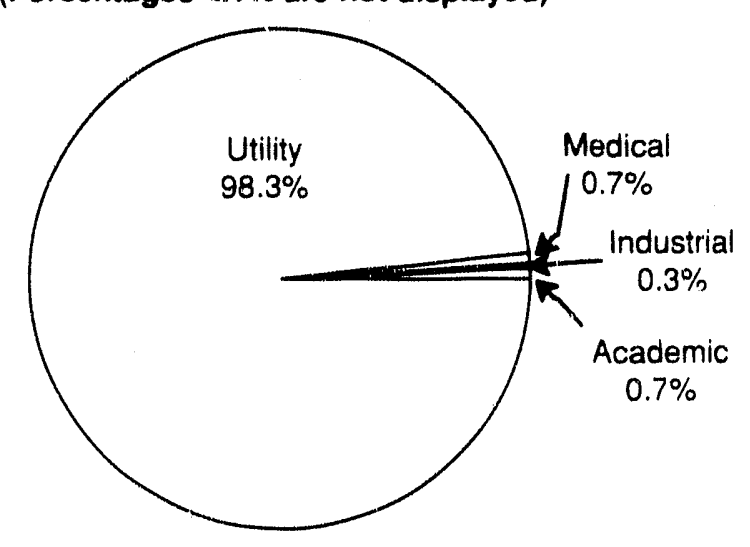

ACTIVITY PERCENTAGE BY SOURCE.

(Percentages <.1\% are not displayed)

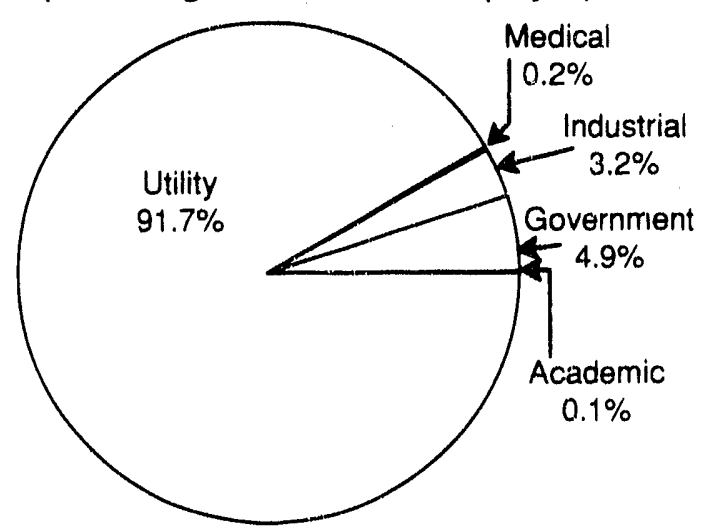




\section{ARKANSAS \\ Low-Level Radioactive Wastes Received at Commercial Disposal Sites}

\begin{tabular}{c} 
Disposal \\
Site \\
\hline
\end{tabular}

Barnwell

Beatty

Richland

Total

$$
\begin{array}{r}
\begin{array}{c}
\text { Volume } \\
\left(\mathrm{ft}^{3}\right)
\end{array} \\
\hline \begin{array}{r}
7,459.85 \\
425.92 \\
84.30
\end{array} \\
\hline 7,970.07
\end{array}
$$

Activity

(curies)

320.18

1.95

0.02

322.16
VOLUME PERCENTAGE BY DISPOSAL SITE (Percentages <. $1 \%$ are not displayed)

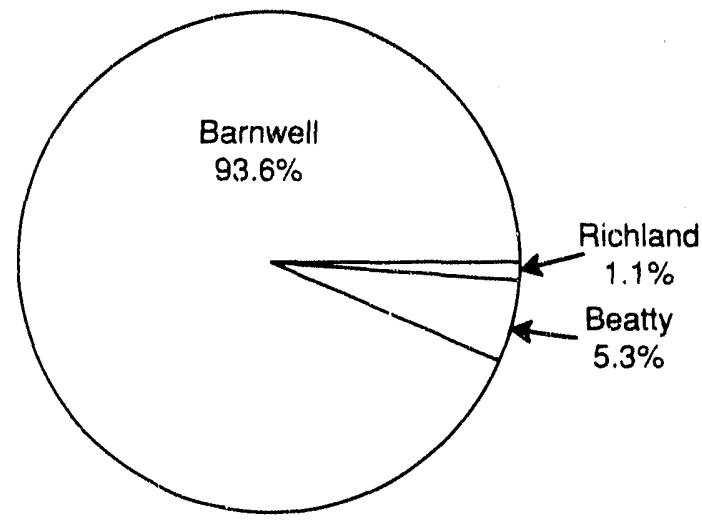

Direct Shipment

Waste Class

A

B

C

Total

Non-Direct Shipments

Grand Total

Total Direct Shipment

Activity (curies)

Total Non-Din:ct Shipment

Activity (curies)

Grand Total (curies)
Volume

$\left(\mathrm{ft}^{3}\right)$

396.00

264.80

0.00

660.80

$7,309.27$

$7,970.07$

282.82

39.33

322.16
ACTIVITY PERCENTAGE BY DISPOSAL SITE

(Percentages <. $1 \%$ are not displayed)

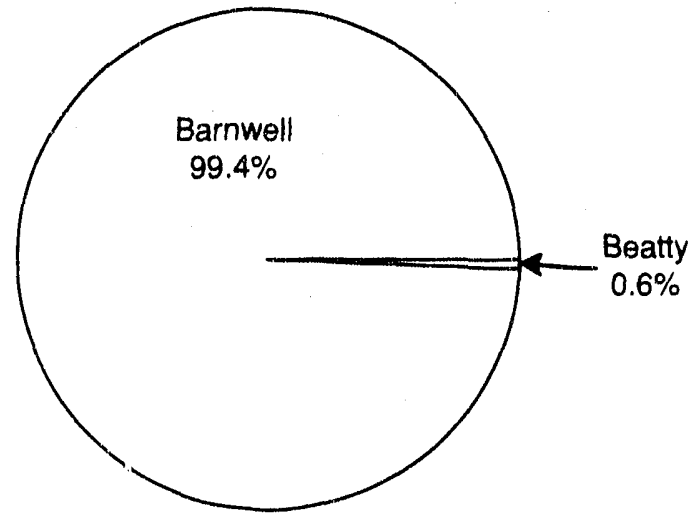

Volume Percentage by Waste Class

for Direct Shipments and Total

Non-Direct Volume Percentage

(Percentages <. $1 \%$ are not displayed)

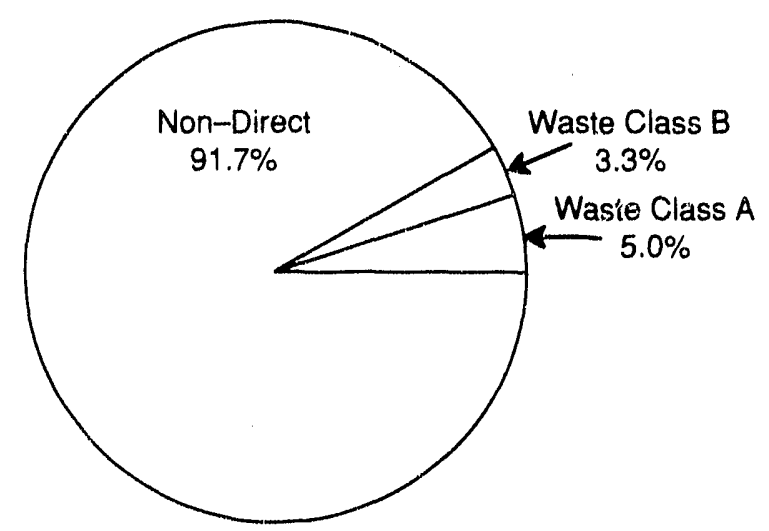




\section{KANSAS \\ Low-Level Radioactive Wastes Received at Commercial Disposal Sites}

\begin{tabular}{l} 
Generator \\
Category \\
\hline
\end{tabular}

Academic

Government

Industrial

Utility

Total
Volume

$\left(\mathrm{ft}^{3}\right)$

148.87

0.30

878.82

$5,337.40$

$6,365.39$
Activity

(curies)

1.38

2.31

$1,208.02$

$1,259.50$

$2,471.22$
VOLUME PERCENTAGE BY SOURCE (Percentages <. $1 \%$ are not displayed)

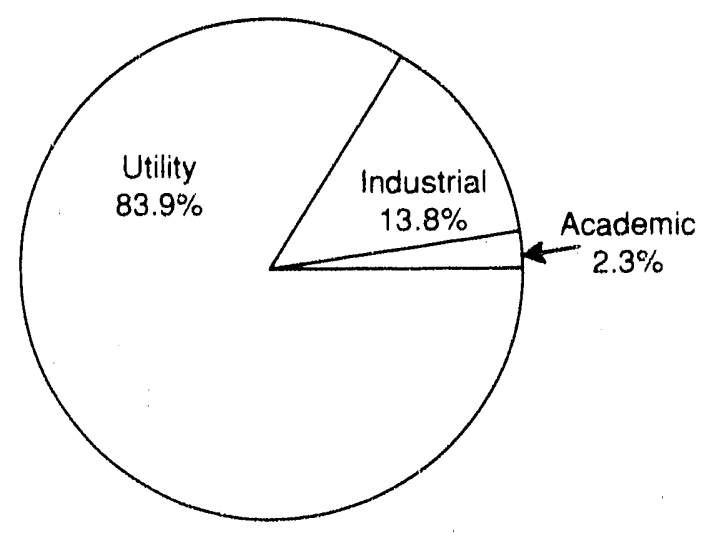

ACTIVITY PERCENTAGE BY SOURCE

(Percentages <. $1 \%$ are not displayed)

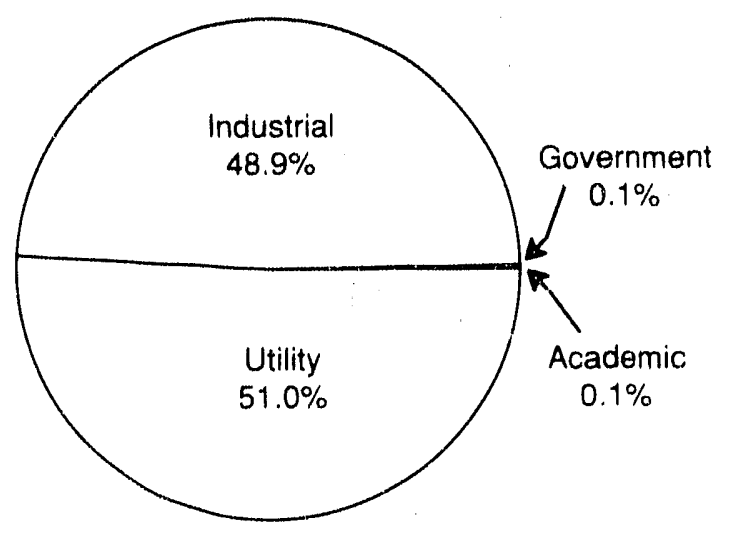




\section{KANSAS \\ Low-Level Radioactive Wastes Received at Commercial Disposal Sites}

\begin{tabular}{c}
$\begin{array}{c}\text { Disposal } \\
\text { Site }\end{array}$ \\
\hline
\end{tabular}

Barnwell

Beatty

Richland

Total

$\begin{array}{r}\begin{array}{c}\text { Volume } \\ \left(\mathrm{ft}^{3}\right)\end{array} \\ \hline 5,337.70 \\ 137.00 \\ 890.69 \\ \hline 6,365.39\end{array}$

Activity

(curies)

$1,261.81$

994.35

215.06

$2,471.22$
VOL_UME PERCENTAGE BY DISPOSAL SITE

(Percentages <. $1 \%$ are not displayed)

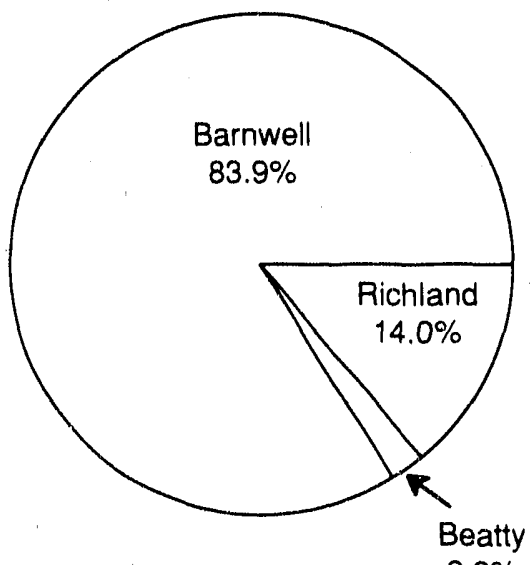

$2.2 \%$

Direct Shipment

Waste Class

A
B
C

Total

Non-Direct Shipments

Grand Total

Total Direct Shipment

Activity (curies)

Total Non-Direct Shipment

Activity (curies)

Grand Total (curies)
$1,211.71$

Volume

$$
\left(\mathrm{ft}^{3}\right)
$$

$4,891.00$

446.40

0.00

$5,337.40$

$1,027.99$

$6,365.39$

$1,259.50$

$2,471.22$
ACTIVITY PERCENTAGE BY DISPOSAL S': : (Percentages <.1\% are not displayed)

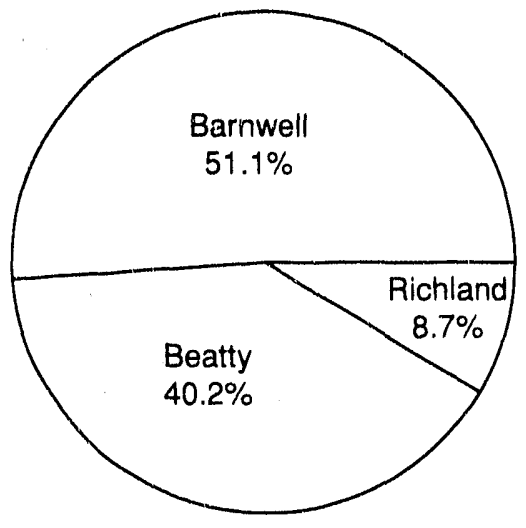

Volume Percentage by Waste Class for Direct Shipments and Total Non-Direct Volume Percentage (Percentages <. $1 \%$ are not displayed)

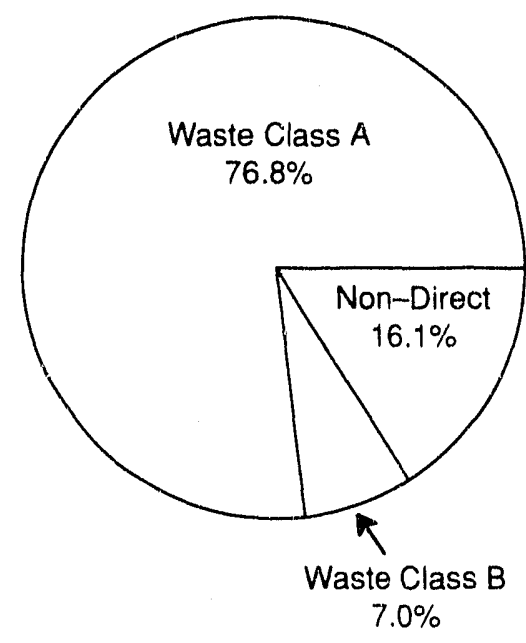




\section{LOUISIANA \\ Low-Level Radioactive Wastes Received at Commercial Disposal Sites}

\begin{tabular}{l} 
Generator \\
Category \\
\hline
\end{tabular}

Academic

Government

Industrial

Medical

Utility

Total

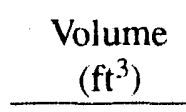

41.18

34.19

361.56

48.58

$21,237.70$

$21,723.21$
Activity

(curies)

$\begin{array}{r}0.23 \\ 225.20 \\ 1.84 \\ 0.40 \\ 1,250.47 \\ \hline 1,478.13\end{array}$

VOLUME PERCENTAGE BY SOURCE

(Percentages <. $1 \%$ are not displayed)

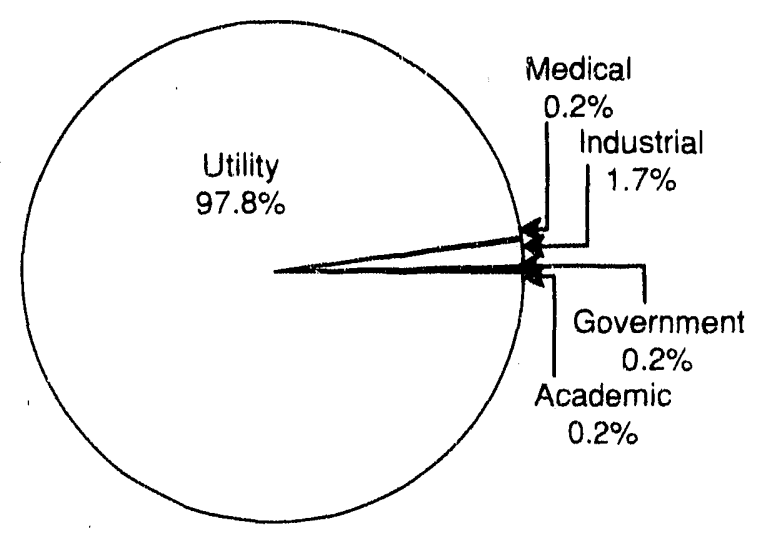

ACTIVITY PERCENTAGE BY SOURCE

(Percentagiss <. 1\% are not displayed)

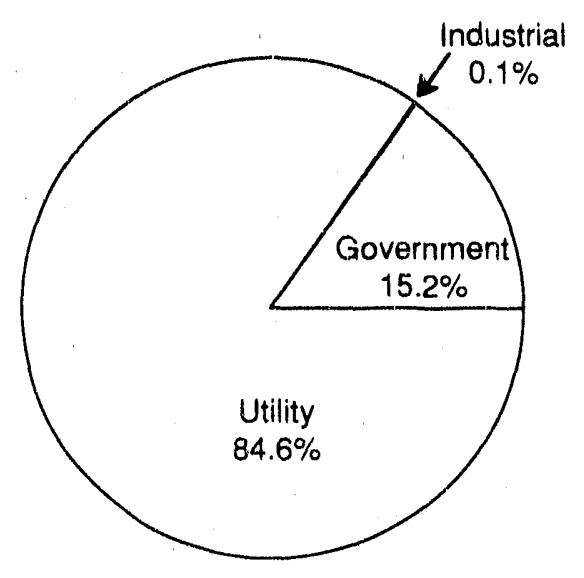




\section{LOUISIANA \\ Low-Level Radioactive Wastes Received at Commercial Disposal Sites}

\begin{tabular}{c} 
Disposal \\
Site \\
\hline
\end{tabular}

Barnwell

Beatty

Richland

Total

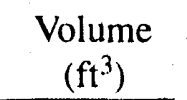

$21,074.00$

57.14

592.07

$21,723.21$
Activity

(curies)

$1,354.97$

0.74

122.43

$1,478.13$
VOLUME PERCENTAGE BY DISPOSAL SITE

(Percentages $<.1 \%$ are not displayed)

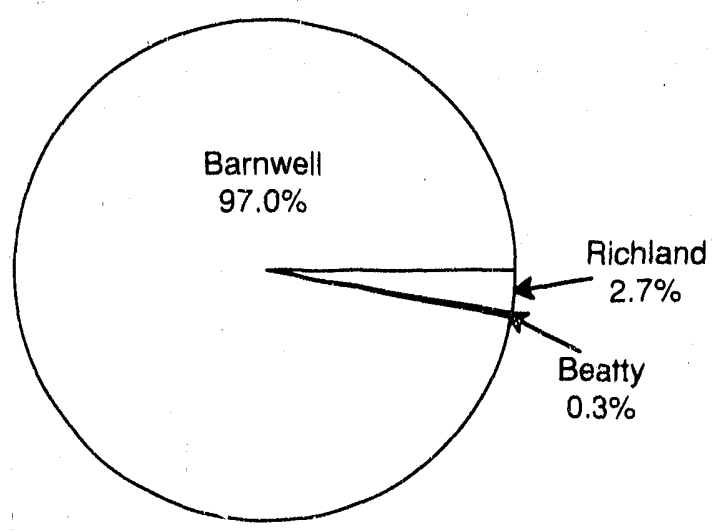

Direct Shipment

Waste Class

A

B

C

Total

Non-Direct Shipments

Grand Total

Total Direct Shipment

Artivity (curies)

Tout Jon-Direct Shipment

Activity (curies)

Grand Total (c ries)
Volume

$\left(\mathrm{ft}^{3}\right)$

$14,393.70$

364.10

120.30

$14,878.10$

$6,845.11$

$21,723.21$

$1,243.35$

234.78

$1,478.13$
ACTIVITY PERCENTAGE BY DISPOSAL SITE

(Percentages <. $1 \%$ are not displayed)

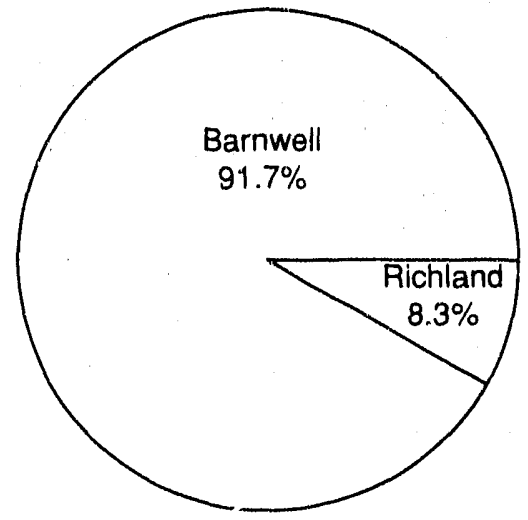

Volume Percentage by Waste Class for Direct Shipments and Total Non-Direct Volume Perceistage (Percentages <. $1 \%$ are not displayed)

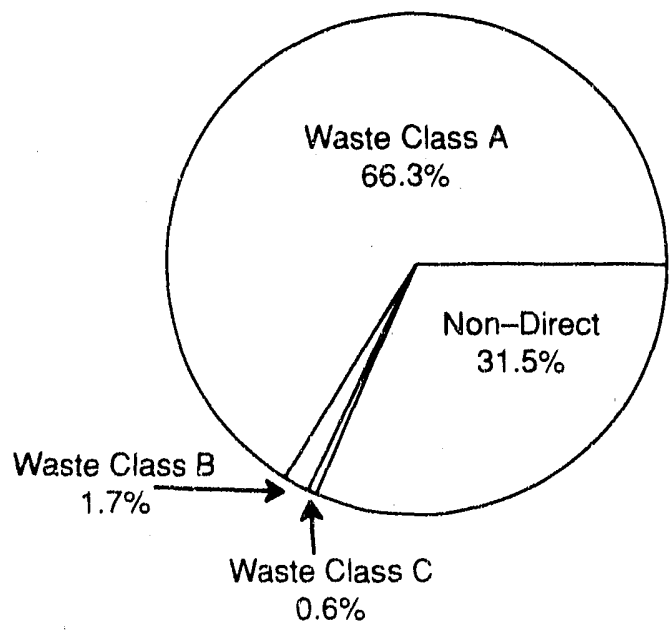




\section{NEBRASKA \\ Low-Level Radioactive Wastes Received at Commercial Disposal Sites}

\section{Generator Category}

Academic

Government

Industrial

Utility

Total

$\begin{array}{r}\begin{array}{c}\text { Volume } \\ \left(\mathrm{ft}^{3}\right)\end{array} \\ \hline 22.50 \\ 0.30 \\ 144.35 \\ 16,532.00 \\ \hline\end{array}$

$16,699.15$
Activity

(curies)

$\begin{array}{r}0.56 \\ 10.69 \\ 1.60 \\ 315.42 \\ \hline 328.26\end{array}$

VOLUME PERCENTAGE BY SOURCE (Percentages $<.1 \%$ are not displayed)

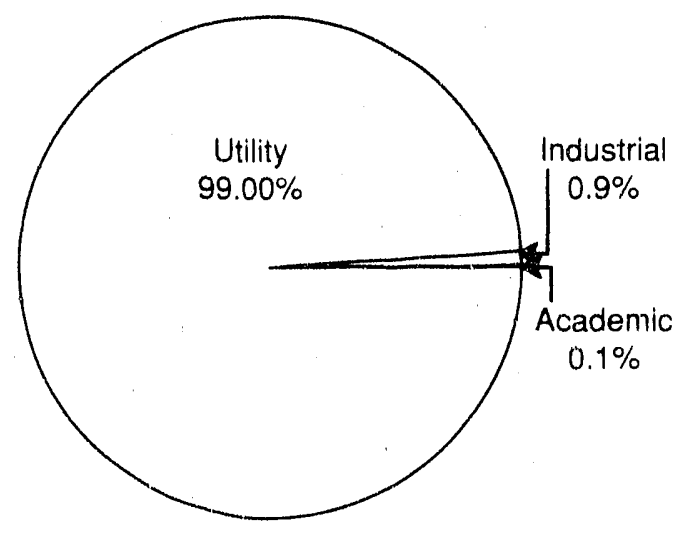

ACTIVITY PERCENTAGE BY SOURCE

(Percentages <. $1 \%$ are not displayed)

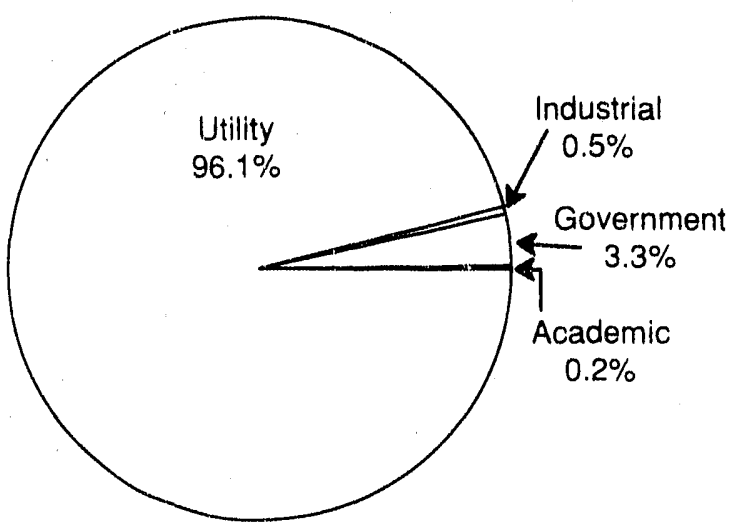




\section{NEBRASKA \\ Low-Level Radioactive Wastes Received at Commercial Disposal Sites}

\begin{tabular}{|c|c|c|}
\hline $\begin{array}{c}\text { Disposal } \\
\text { Site } \\
\end{array}$ & $\begin{array}{c}\text { Volume } \\
\left(\mathrm{ft}^{3}\right)\end{array}$ & $\begin{array}{l}\text { Activity } \\
\text { (curies) }\end{array}$ \\
\hline Barnwell & 0.30 & 10.69 \\
\hline Beatty & 230.45 & 98.66 \\
\hline Richland & $16,468.40$ & 218.91 \\
\hline Total & $16,699.15$ & 328.26 \\
\hline
\end{tabular}

VOL.UME PERCENTAGE BY DISPOSAL SITE

(Percentages <.1\% are not displayed)

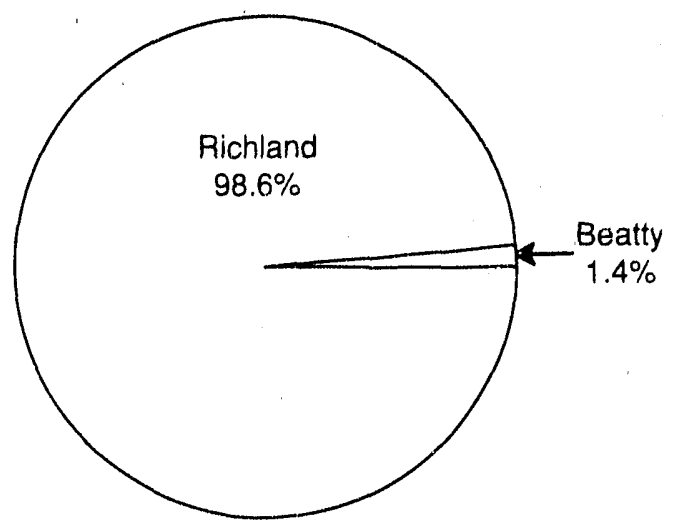

Direct Shipment

Waste Class

A

B

C

Total

Non-Direct Shiprnents

Grand Total

Total Direct Shipment

Activity (curies)

Total Non-Direci Shipment

Activity (curies)

Grand Total (curies) Volume $\left(\mathrm{ft}^{3}\right)$

$\begin{array}{r}16,382.00 \\ 150.00 \\ 0.00 \\ \hline 16,532.00 \\ \hline 167.15 \\ \hline 16,699.15 \\ 12.85 \\ \hline 328.26\end{array}$

ACTIVITY PERCENTAGE BY DISPOSAL SITE

(Percentages $<.1 \%$ are not displayed)

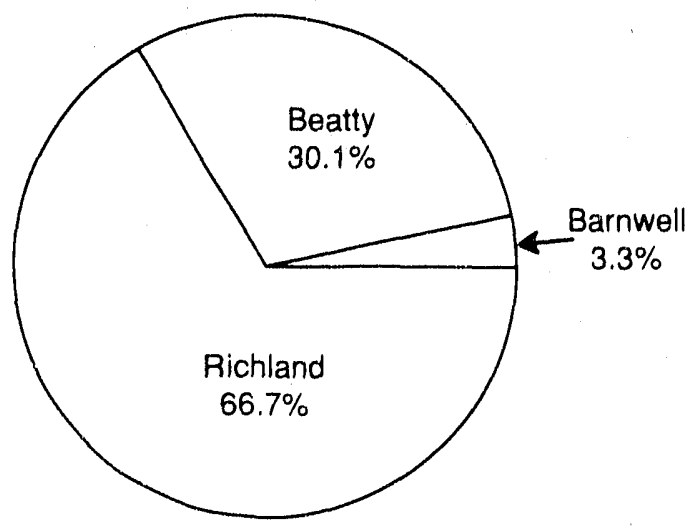

Volume Percentage by Waste Class for Direct Shipments and Total Non-Direct Volume Percentage (Percentages $<.1 \%$ are nol displayed

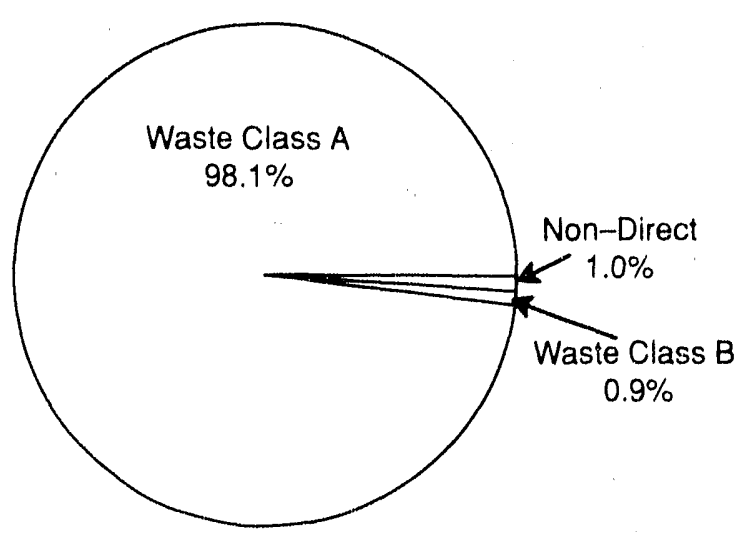




\section{OKLAHOMA \\ Low-Level Radioactive Wastes Received at Commercial Disposal Sites}

\begin{tabular}{l} 
Generator \\
Category \\
\hline
\end{tabular}

Academic

Government

Industrial

Medical

Total

$\begin{array}{r}\begin{array}{c}\text { Volume } \\ \left(\mathrm{ft}^{3}\right)\end{array} \\ \hline 45.30 \\ 652.60 \\ 31,406.53 \\ 22.50 \\ \hline\end{array}$

$32,126.93$
Activity

(curies)

0.17

0.01

4.05

0.01

4.25
VOLUME PERCENTAGE BY SOURCE (Percentages <.1\% are not displayed)

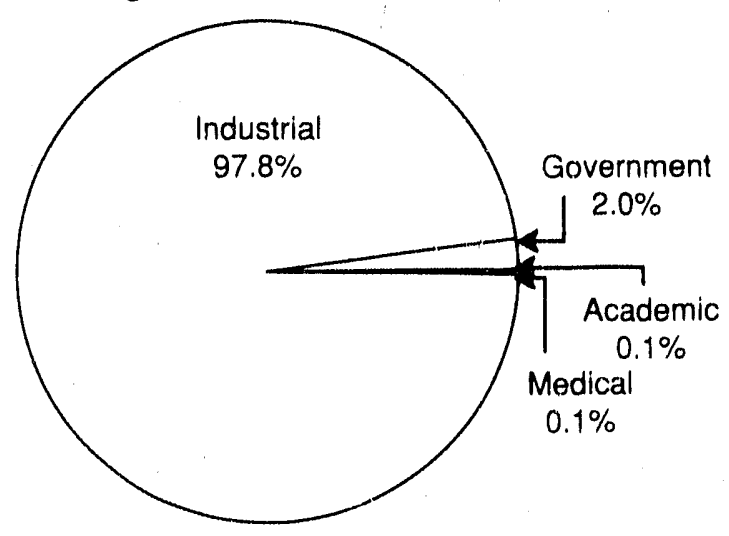

ACTIVITY PERCENTAGE BY SOURCE

(Percentages $<.1 \%$ are not displayed)

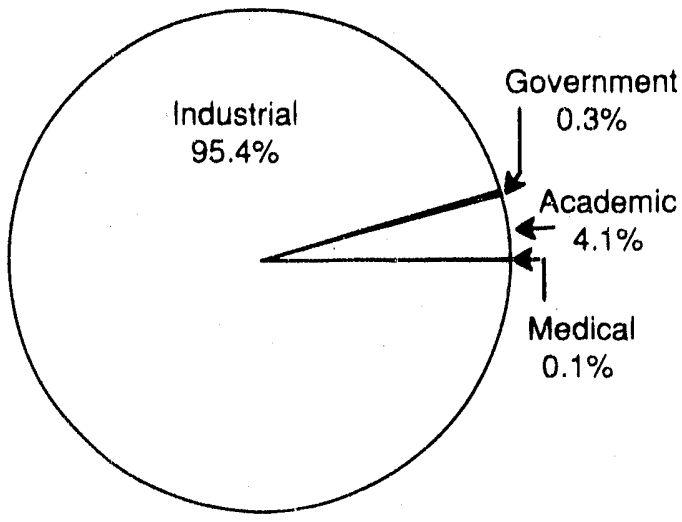




\section{OKLAHOMA \\ Low-Level Radioactive Wastes Received at Commercial Disposal Sites}

\begin{tabular}{c}
$\begin{array}{c}\text { Disposal } \\
\text { Site }\end{array}$ \\
\hline
\end{tabular}

Barnwell

Beatty

Richland

Total

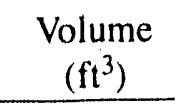

$25,192.60$

$2,881.19$

$4,053.14$

$32,126.93$
Activity

(curies)

0.96

0.87

2.41

4.25
VOLUME PERCENTAGE BY DISPOSAL SITE (Percentages <. $1 \%$ are not displayed)

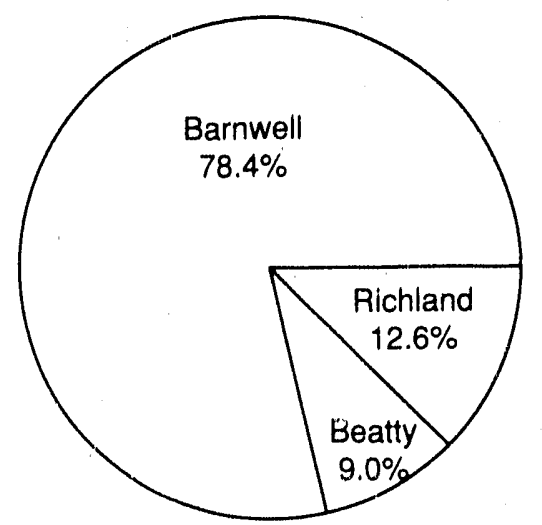

Direct Shipment

Waste Class

A

B

C

Total

Non-Direct Shipments

Grand Total

Total Direct Shipment

Activity (curies)

Total Non-Direct Shipment

Activity (curies)

Grand Total (curies)
ACTIVITY PERCENTAGE BY DISPOSAL SITE (Percentages <.1\% are not displayed)
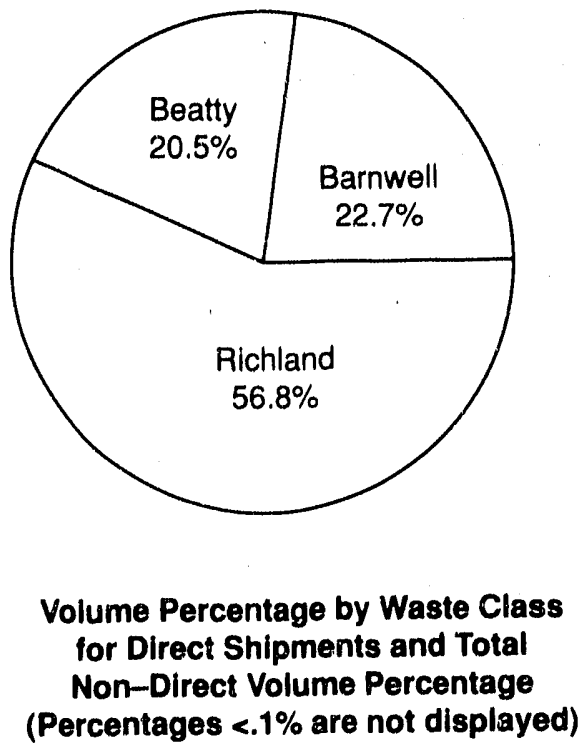

Volume $\left(\mathrm{ft}^{3}\right)$

$30,930.00$

0.00

0.00

$30,930.00$

$1,196.93$

$32,126.93$

3.48

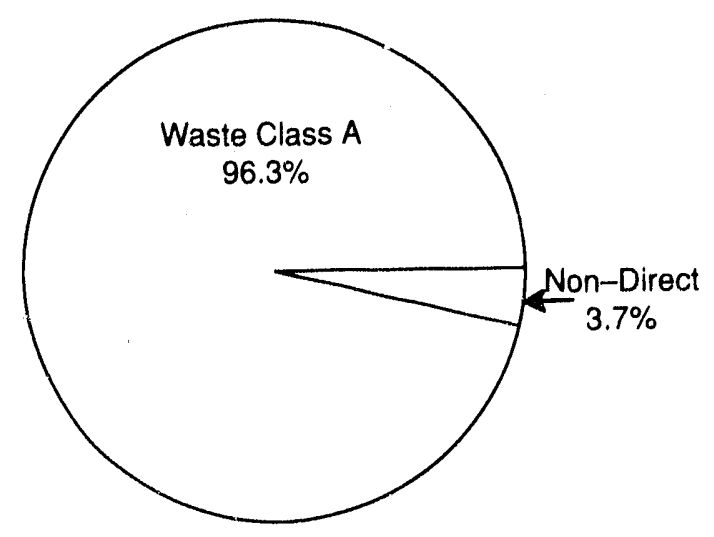

0.77

4.25 


\section{CENTRAL MIDWEST COMPACT \\ Low-Level Radioactive Wastes Received at Commercial Disposal Sites}

\begin{tabular}{lrr}
\multicolumn{1}{c}{ States } & \multicolumn{1}{c}{$\begin{array}{c}\text { Volume } \\
\left(\mathrm{ft}^{3}{ }^{3}\right.\end{array}$} & \multicolumn{1}{c}{$\begin{array}{c}\text { Activity } \\
\text { (curies) }\end{array}$} \\
\cline { 2 - 3 } Illinois & $\begin{array}{r}134,786.89 \\
10,047.07\end{array}$ & $\begin{array}{r}147.114 .64 \\
21.09\end{array}$ \\
Kentucky & $144,833.96$ & $147,135.72$
\end{tabular}

VOLUME PERCENTAGE BY STATE

(Percentages $<.1 \%$ are not displayed)

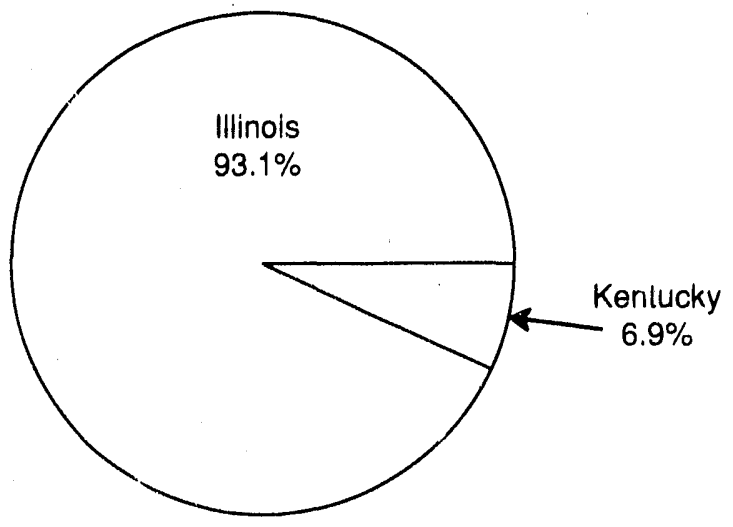

VOLUME PERCENTAGE BY CATEGORY

(Percentages $<.1 \%$ are not displayed)

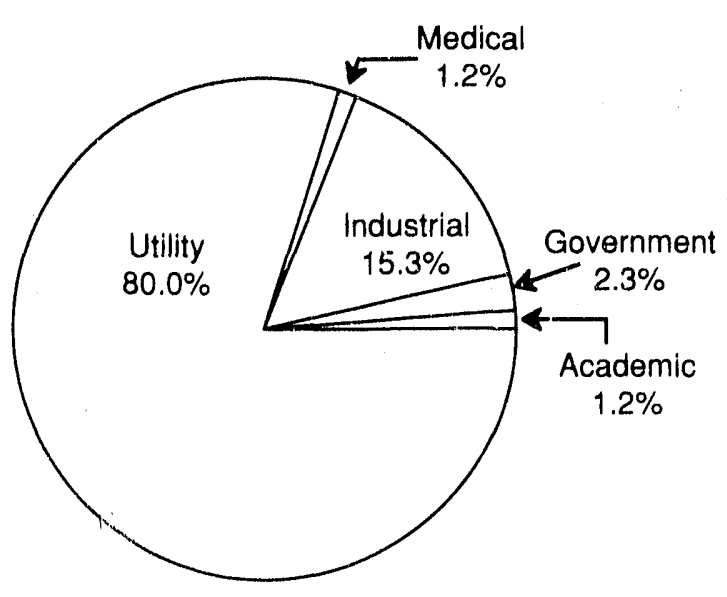




\section{ILLINOIS \\ Low-Level Radioactive Wastes Recrived at Commercial Disposal Sites}

\begin{tabular}{l} 
Generator \\
Category \\
\hline
\end{tabular}

Academic

Government

Industrial

Medical

Utility

Total

Volume
$\left(\mathrm{ft}^{3}\right)$

$1,156.93$

$3,357.66$

$12,733.57$

$1,660.85$

$115,877.88$

$134,786.89$
Activity

(curies)

2.71

1.06

55.60

4.15

$147,051.13$

$147,114.64$
VOLUME PERCENTAGE BY SOURCE

(Percentages <.1\% are not displayed)

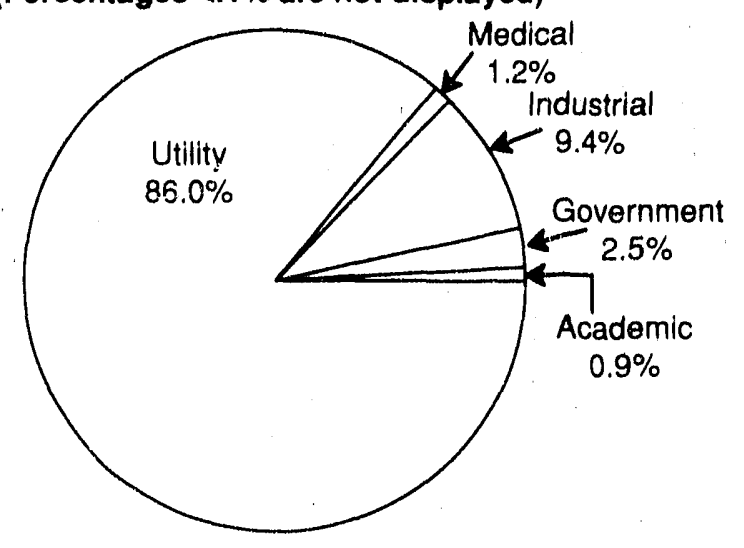

ACTIVITY PERCENTAGE BY SOURCE

(Percentages <.1\% are not displayed)

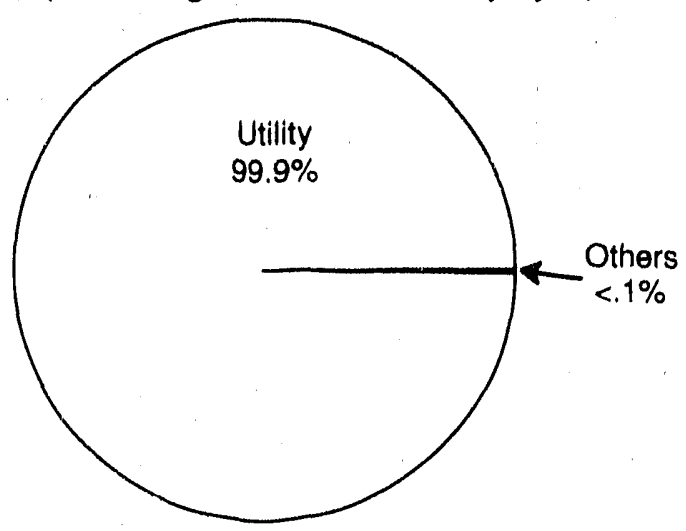




\section{ILLINOIS \\ Low-Level Radioactive Wastes Received at Commercial Disposal Sites}

\begin{tabular}{c} 
Disposal \\
Site \\
\hline
\end{tabular}

Barnwell

Beatty

Richland

Total

$$
\begin{gathered}
\begin{array}{c}
\text { Volume } \\
\left(\mathrm{ft}^{3}\right)
\end{array} \\
\hline 87,641.29 \\
12,371.82 \\
34,773.78 \\
\hline
\end{gathered}
$$

$134,786.89$
Activity

(curies)

$142,210.84$

$3,491.53$

$1,412.27$

$147,114.64$
VOLUME PERCENTAGE BY DISPOSAL SITE

(Percentages <.1\% are not displayed)

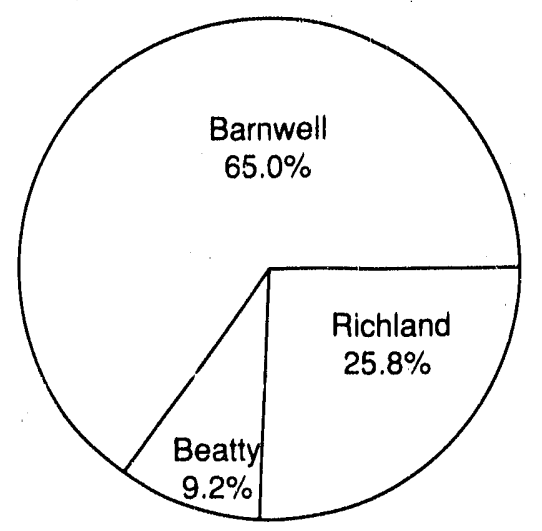

ACTIVITY PERCENTAGE BY DISPOSAL SITE

(Percentages <. $1 \%$ are not displayed)

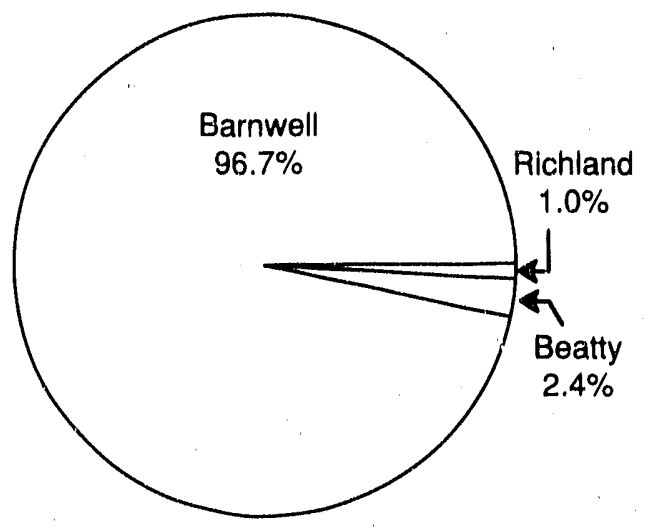

Volume Percentage by Waste Class for Direct Shipments and Total Non-Direct Volume Percentage (Percentages <. $1 \%$ are not displayed)
Direct Shipment

Waste Class

\section{A}

B

C

Total

Non-Direct Shipments

Grand Total

Total Direct Shipment

Activity (curies)

Total Non-Direct Shipment

Activity (curies)

Grand Total (curies)
Volume

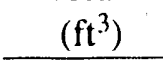

$74,777.90$

$3,543.50$

$2,487.20$

$80,808.60$

$53,978.29$

$134,786.89$

$146,982.92$

131.72

$147,114.64$ 


\section{KENTUCKY \\ Low-Level Radioactive Wastes Received at Commercial Disposal Sites}

\begin{tabular}{l} 
Generator \\
Category \\
\hline
\end{tabular}

Academic

Government

Industrial

Medical

Total

$\begin{array}{r}\begin{array}{c}\text { Volume } \\ \left(\mathrm{ft}^{3}\right)\end{array} \\ \hline \begin{array}{r}623.40 \\ 2.60 \\ 9,409.21 \\ 11.86\end{array} \\ \hline 10,047.07\end{array}$

Activity

(curies)

1.27

14.96

4.73

0.12

21.09
VOLUME PERCENTAGE BY SOURCE (Percentages <. $1 \%$ are not displayed)

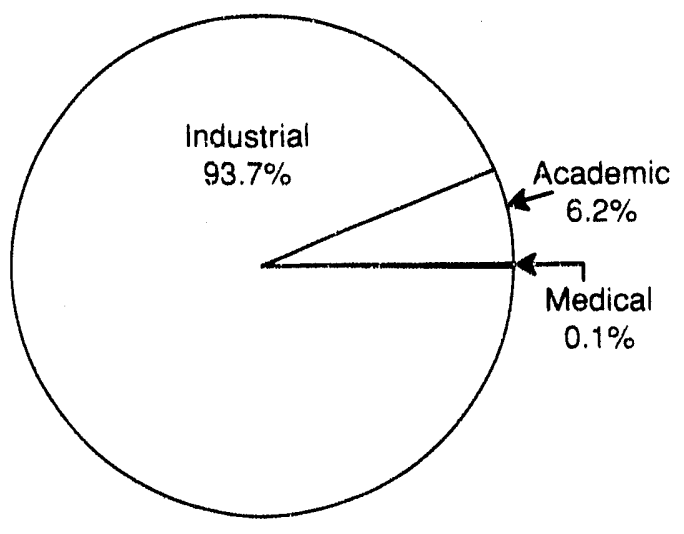

ACTIVITY PERCENTAGE BY SOURCE

(Percentages <.1\% are not displayed)

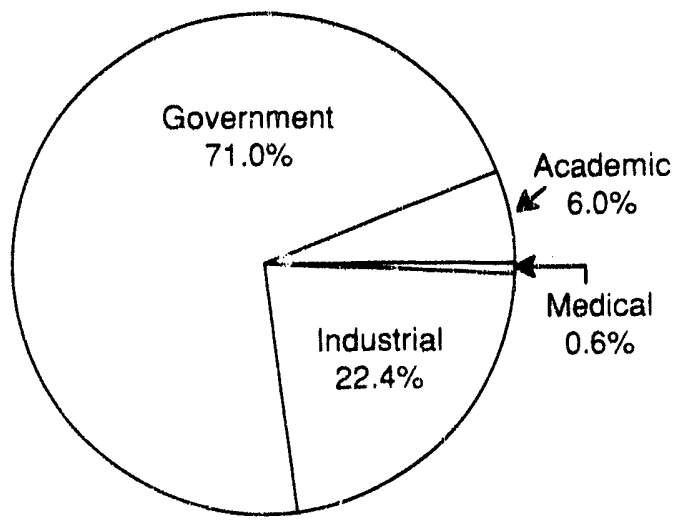




\section{KENTUCKY \\ Low-Level Radioactive Wastes Received at Commercial Disposal Sites}

\begin{tabular}{lrr}
\multicolumn{1}{c}{$\begin{array}{c}\text { Disposal } \\
\text { Site }\end{array}$} & \multicolumn{1}{c}{$\begin{array}{c}\text { Volume } \\
\left(\mathrm{ft}^{3}\right)\end{array}$} & \multicolumn{1}{c}{$\begin{array}{c}\text { Activity } \\
\text { (curies) }\end{array}$} \\
\cline { 2 - 3 } Barnwell & & 15.07 \\
Beatty & 444.60 & 0.62 \\
Richland & 561.57 & 5.39 \\
Total & $9,040.90$ & 21.09
\end{tabular}

VOLUME PERCENTAGE BY DISPOSAL SITE

(Percentages $<.1 \%$ are not displayed)

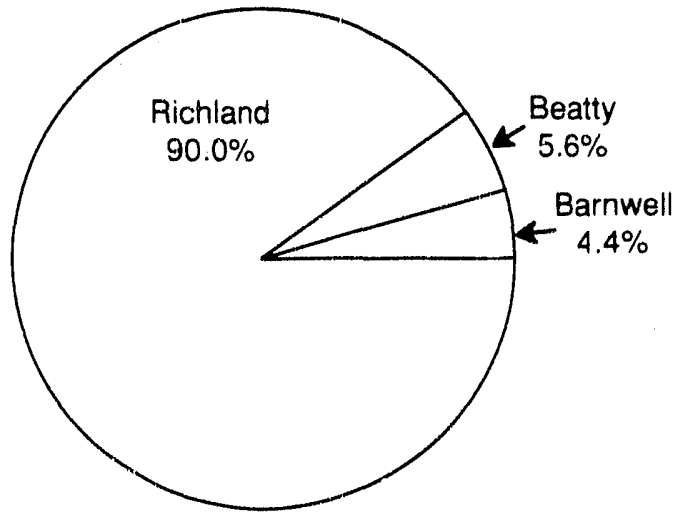

Direct Shipment

Waste Class

A

B

C

Total

Non-Direct Shipments

Grand Total

Total Ditect Shipment

Activity (curies)

Total Non-Direct Shipment

Activity (curies)

Grand Total (curies)

Volume

$\left(\mathrm{ft}^{3}\right)$

$\begin{array}{r}15.00 \\ 0.00 \\ 0.00 \\ \hline 15.00 \\ \hline 10,032.07 \\ \hline 10,047.07 \\ 0.00 \\ 21.09 \\ \hline 21.09\end{array}$

ACTIVITY PERCENTAGE BY DISPOSAL SITE

(Percentages <. $1 \%$ are not displayed)

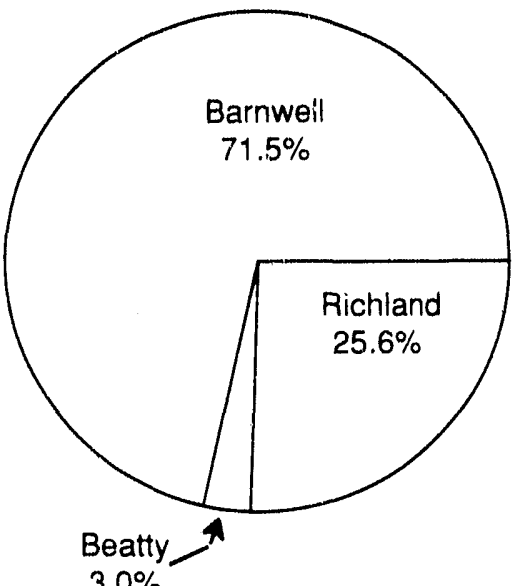

Volume Percentage by Waste Class

for Direct Shipments and Total

Non-Direct Volume Percentage

(Percentages <. $1 \%$ are not displayed)

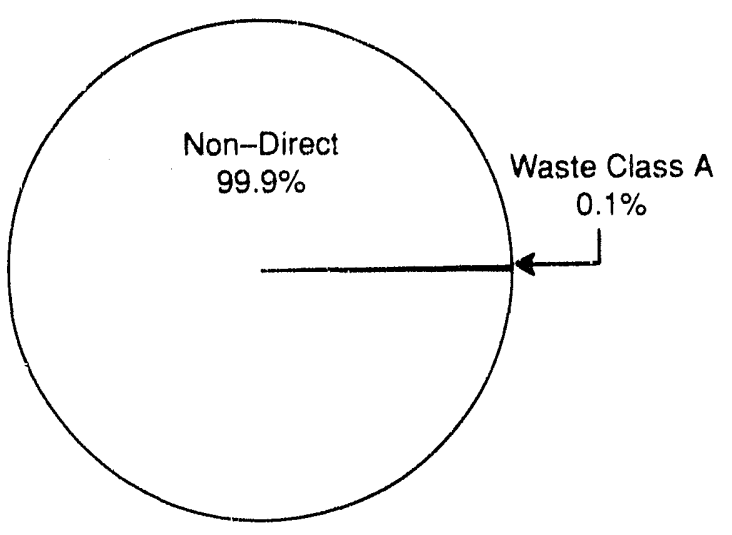




\section{MIDWEST COMPACT \\ Low-Level Radioactive Wastes Received at Commercial Disposal Sites}

\begin{tabular}{lrr}
\multicolumn{1}{c}{ States } & \multicolumn{1}{c}{$\begin{array}{c}\text { Volume } \\
\left(\mathrm{ft}^{3}\right)\end{array}$} & \multicolumn{1}{c}{$\begin{array}{c}\text { Activity } \\
\text { (curies) }\end{array}$} \\
Indiana & $2,150.31$ & 63.13 \\
Iowa & $6,055.37$ & $16,953.42$ \\
Michigan & $43,111.39$ & $7,185.79$ \\
Minnesota & $21,954.29$ & $60,952.08$ \\
Missouri & $18,802.64$ & 793.03 \\
Ohio & $58,866.18$ & $1,211.40$ \\
Wisconsin & $6,880.93$ & $1,074.88$ \\
& & $88,233.74$
\end{tabular}

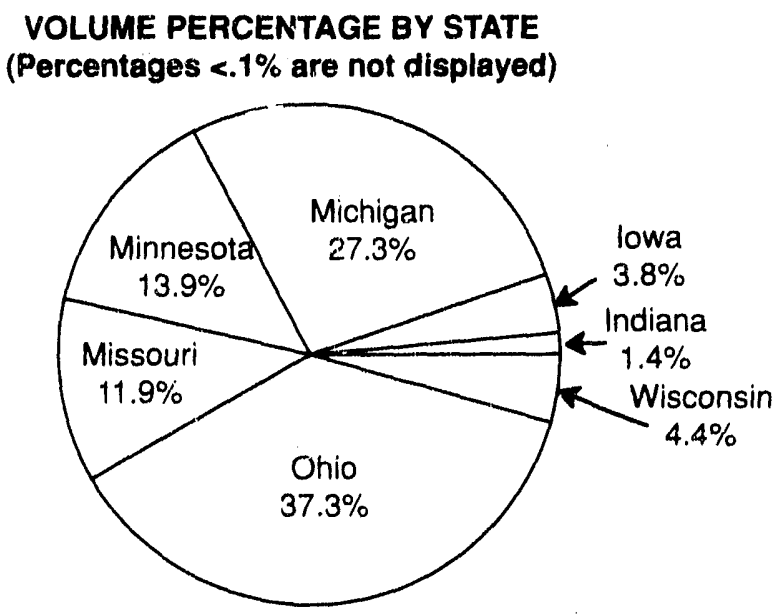

VOLUME PERCENTAGE BY CATEGORY

(Percentages <. $1 \%$ are not displayed)

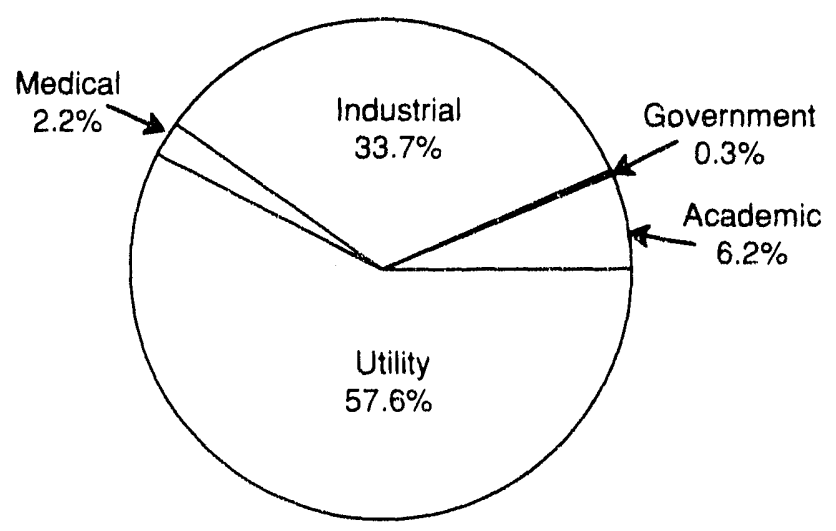




\section{IOWA \\ Low-Level Radioactive Wastes Received \\ at Commercial Disposal Sites}

\begin{tabular}{lrr}
$\begin{array}{c}\text { Generator } \\
\text { Category }\end{array}$ & \multicolumn{1}{c}{$\begin{array}{c}\text { Volume } \\
\left(\mathrm{ft}^{3}\right)\end{array}$} & $\begin{array}{r}\text { Activity } \\
\text { (curies) }\end{array}$ \\
\cline { 2 - 2 } & 169.35 & 0.19 \\
Academic & 5.70 & 3.39 \\
Government & 804.72 & 1.29 \\
Industrial & $5,075.60$ & $16,948.55$ \\
Utility & $6,055.37$ & $16,953.42$
\end{tabular}

VOLUME PERCENTAGE BY SOURCE

(Percentages <. $1 \%$ are not displayed)

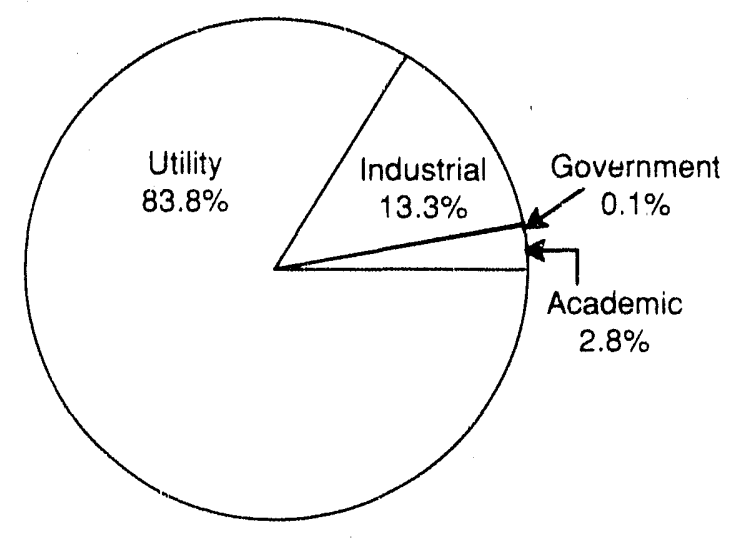

ACTIVITY PERCENTAGE BY SOURCE

(Percentages <. $1 \%$ are not displayed)

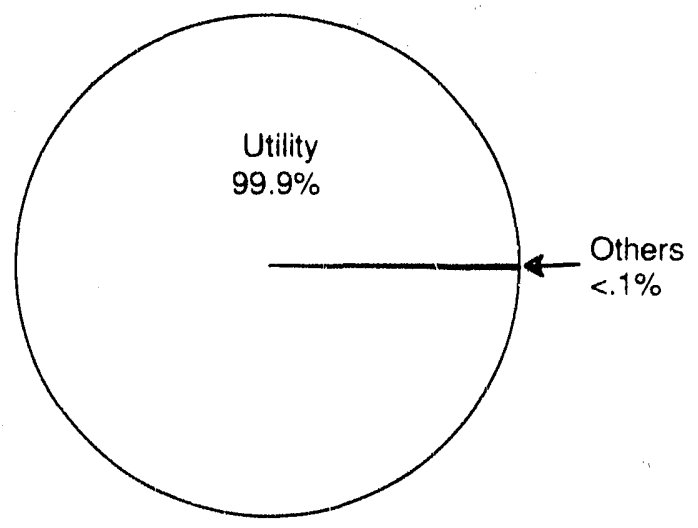




\section{IOWA \\ Low-Level Radioactive Wastes Received at Commercial Disposal Sites}

\begin{tabular}{|c|c|c|}
\hline $\begin{array}{l}\text { Disposal } \\
\text { Site }\end{array}$ & $\begin{array}{c}\text { Volume } \\
\left(\mathrm{ft}^{3}\right)\end{array}$ & $\begin{array}{l}\text { Activity } \\
\text { (curies) }\end{array}$ \\
\hline Barnwell & $3,719.50$ & $16,904.51$ \\
\hline Beatty & 14.72 & 1.26 \\
\hline Richland & $2,321.15$ & 47.65 \\
\hline Total & $6,055.37$ & $16,953.42$ \\
\hline
\end{tabular}

VOLUME FERCENTAGE BY DISPOSAL SITE (Percentages <.1\% are not displayed)

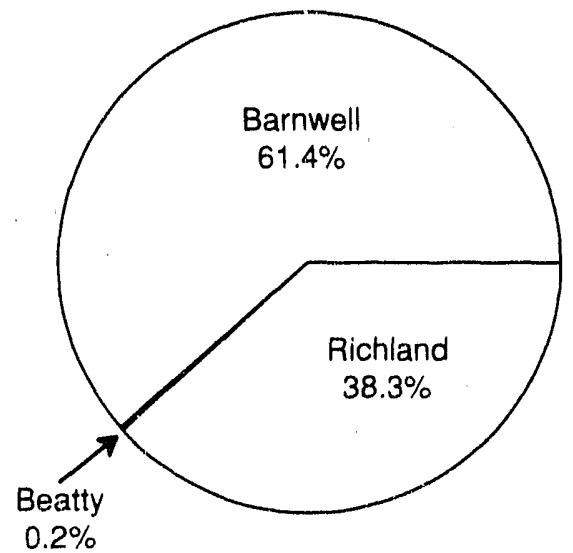

Direct Shipment

Waste Cläss

A

B

C

Total

Non-Direct Shipments

Grand Total

Total Direct Shipment

Activity (curies)

Total Non-Direct Shipment

Activity (curies)

Grand Total (curies)
Volume

$\left(\mathrm{ft}{ }^{3}\right)$

$3,996.00$

135.80

57.50

$4,189.30$

$1,866.07$

$6,055.37$

$16,941.67$

11.75

$16,953.42$

\section{ACTIVITY PERCENTAGE BY DISPOSAL SITE} (Percentages <. $1 \%$ are not displayed)

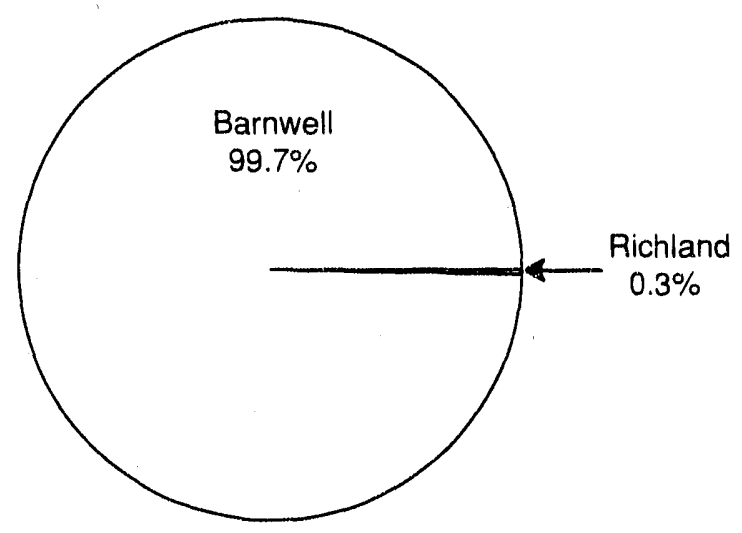

Volume Percentage by Waste Class for Direct Shipments and Total Non-Direct Volume Percentage (Percentages $<.1 \%$ are not displayed)

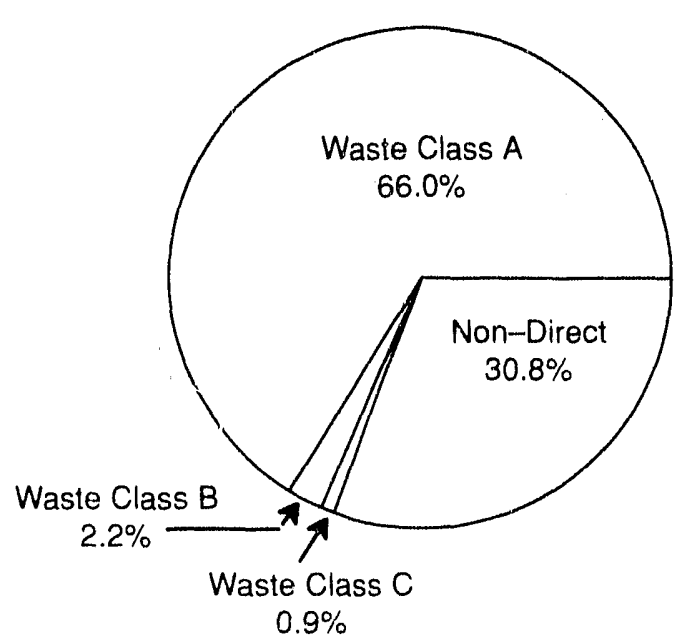




\section{INDIANA \\ Low-Level Radioactive Wastes Received at Commercial Disposal Sites}

\author{
Generator \\ Category \\ Academic \\ Government \\ Industrial \\ Medical \\ Utility
}

Total

$$
\begin{array}{r}
\begin{array}{c}
\text { Volume } \\
\left(\mathrm{ft}^{3}\right)
\end{array} \\
\hline 328.45 \\
10.90 \\
1,782.59 \\
21.80 \\
6.57 \\
\hline 2,150.31
\end{array}
$$

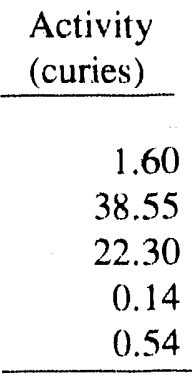

63.13
VOLUME PERCENTAGE BY SOURCE (Percentages <.1\% are not displayed)

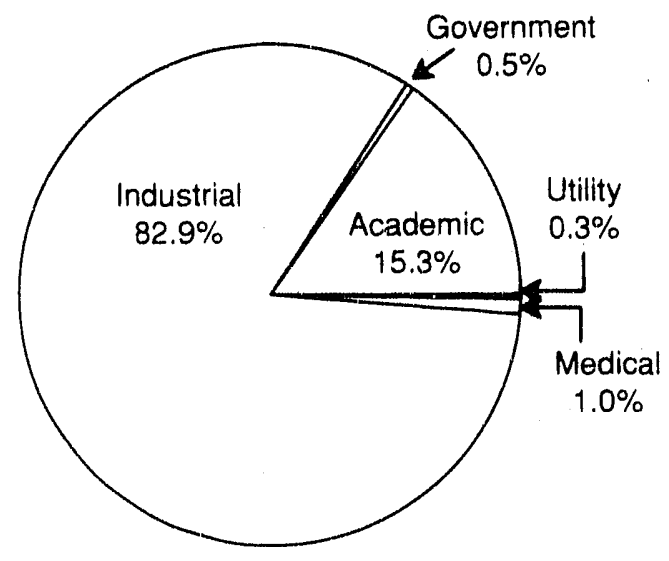

\section{ACTIVITY PERCENTAGE BY SOURCE}

(Percentages <. $1 \%$ are not displayed)

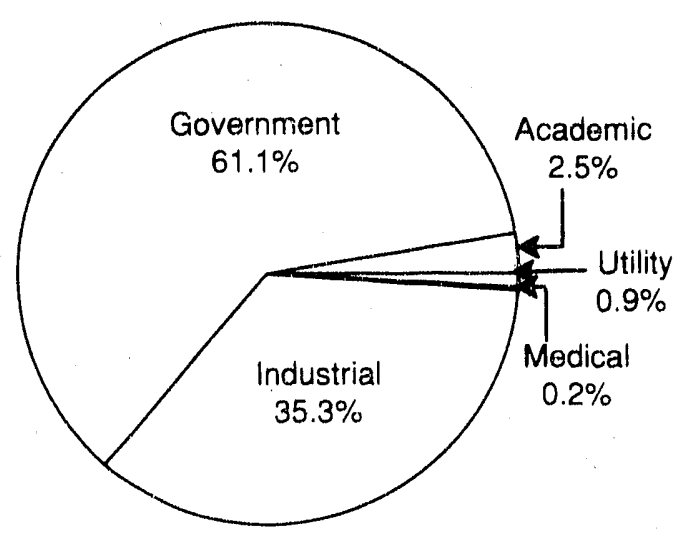




\section{INDIANA \\ Low-Level Radioactive Wastes Received at Commercial Disposal Sites}

\begin{tabular}{c} 
Disposal \\
Site \\
\hline
\end{tabular}

Barnwell

Beatty

Richland

Total

Volume
$\left(\mathrm{ft}^{3}\right)$

$1,437.61$

197.31

515.39

$2,150.31$
Activity

(curies)

43.62

17.86

1.65

63.13

\section{VOLUME PERCENTAGE BY DISPOSAL SITE (Percentages $<.1 \%$ are not displayed)}

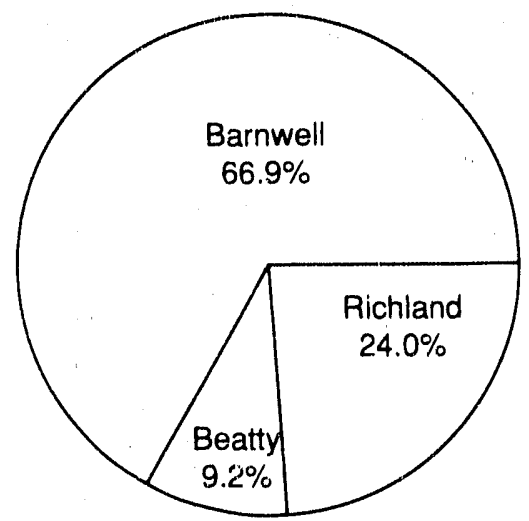

Direct Shipment

Waste Class

$$
\begin{aligned}
& \text { A } \\
& \text { B } \\
& \text { C }
\end{aligned}
$$$$
\text { Total }
$$

Non-Direct Shipments

Grand Total

Total Direct Shipment

Activity (curies)

Total Non-Direct Shipment

Activity (curies)

Volume

$\left(\mathrm{ft}^{3}\right)$

Grand Total (curies)
$1,375.70$

0.00

0.00

$1,375.70$

774.61

$2,150.31$

4.96

$\underline{58.17}$

63.13
ACTIVITY PERCENTAGE BY DISPOSAL SITE

(Percentages <.1\% are not displayed)

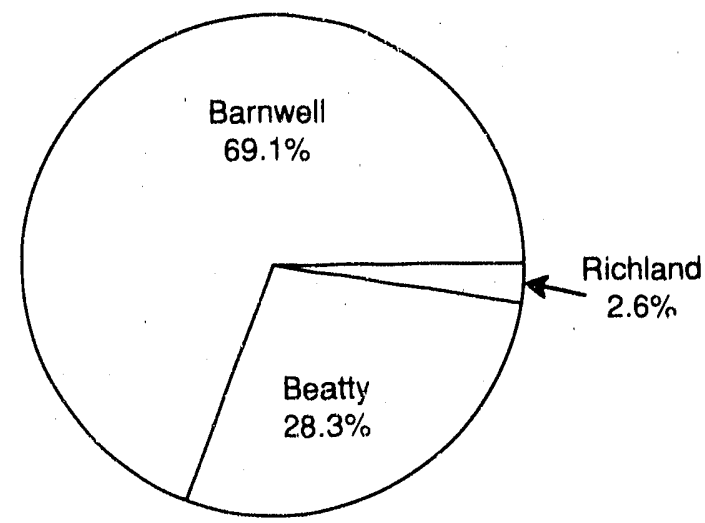

Volume Percentage by Waste Class for Direct Shipments and Total Non-Direct Volume Percentage (Percentages <. $1 \%$ are not displayed)

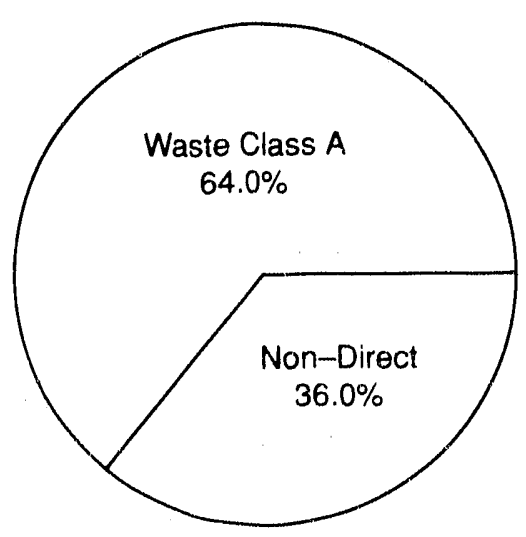




\section{MICHIGAN \\ Low-Level Radioactive Wastes Received \\ at Commercial Disposal Sites}

\begin{tabular}{l} 
Generator \\
Category \\
\hline
\end{tabular}

Academic

Government

Industrial

Medical

Utility

Total

$\begin{array}{r}\begin{array}{c}\text { Volume } \\ \left(\mathrm{ft}^{3}\right)\end{array} \\ \hline 3,123.21 \\ 15.35 \\ 2,119.77 \\ 641.00 \\ 37,212.06 \\ \hline\end{array}$

$43,111.39$
Activity

(curies)

$$
\begin{array}{r}
6.40 \\
<1 \\
707.72 \\
0.55 \\
6,471.11 \\
\hline 7,185.79
\end{array}
$$

ACTIVITY PERCENTAGE BY SOURCE

(Percentages <. $1 \%$ are not displayed)
VOLUME PERCENTAGE BY SOUnCE
(Percentages $<.1 \%$ are not displayed)

(Percentages $<.1 \%$ are not displayed)
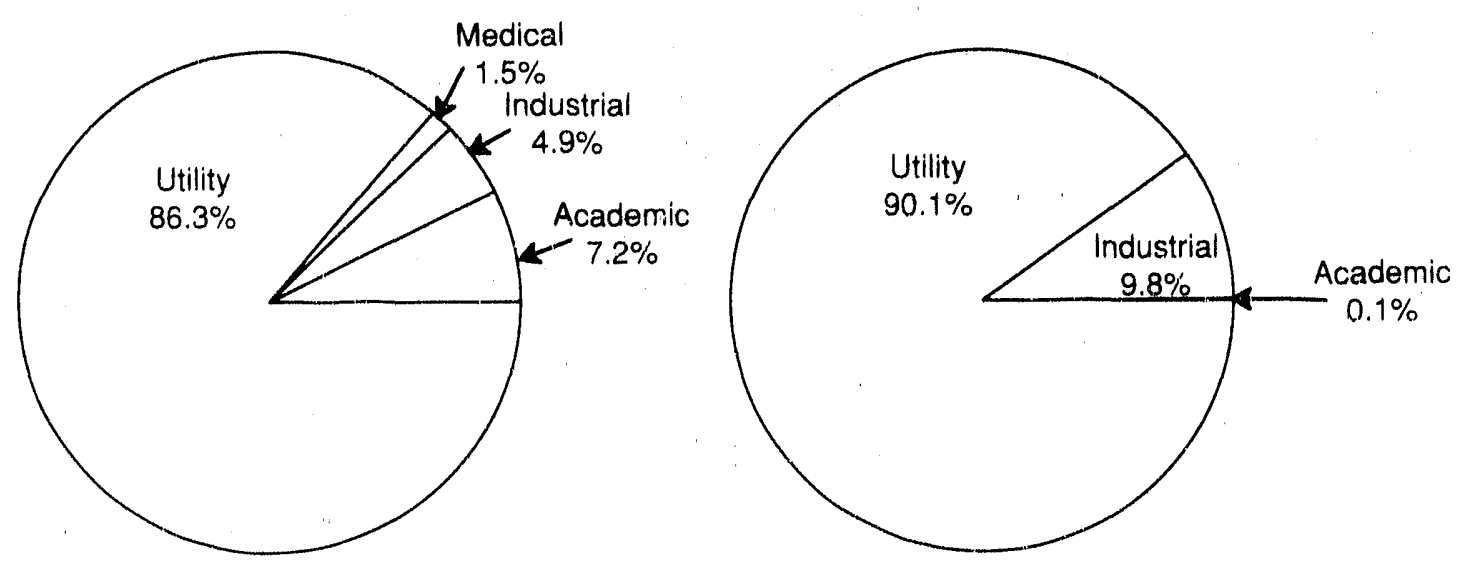


\section{MICHIGAN \\ Low-Level Radioactive Wastes Received at Commercial Disposal Sites}

\begin{tabular}{c} 
Disposal \\
Site \\
\hline
\end{tabular}

Barnwell

Beatty

Richland

Total

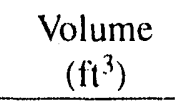

$35,894.03$

$1,038.63$

$6,178.73$

$43,111.39$
Activity

(curies)

$6,465.14$

660.46

60.20

$7,185.79$
VOLUME PERCENTAGE BY DISPOSAL. SITE (Percentages $<.1 \%$ are not displayed)

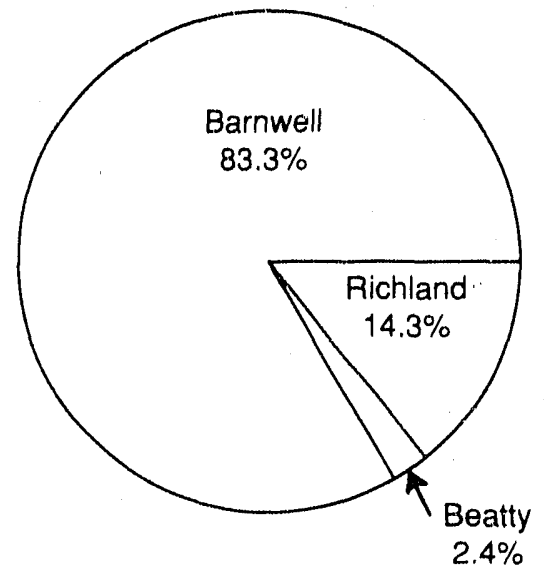

Direct Shipment

Waste Class

A

B

C

Total

Non-Direct Shipments

Grand Total

Total Direct Shipment

Activity (curies)

Total Non-Direct Shipment

Activity (curies)

Grand Total (curies)

$\left(\mathrm{ft}^{3}\right)$

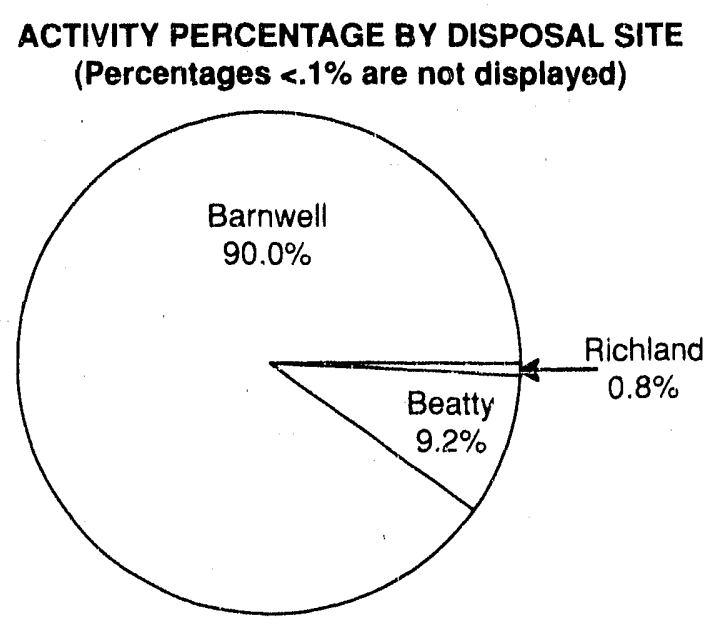

Volume Percentage by Waste Class for Direct Shipments and Total Non-Direct Volume Percentage (Percentages <. $1 \%$ are not displayed)

Waste Class B

$13,988.09$

888.60

$1,491.30$

$16,367.99$

$26,743.40$

$43,111.39$

$6,441.71$

744.08

$7,185.79$

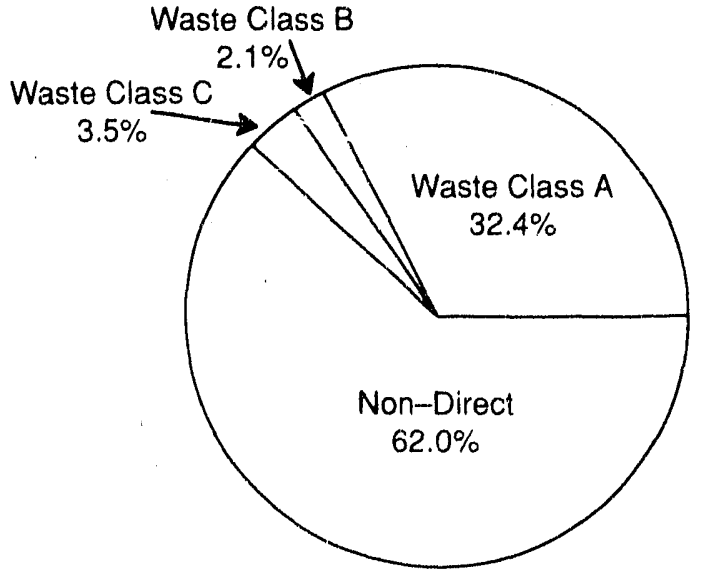




\section{MINNESOTA \\ Low-Level Radioactive Wastes Received \\ at Commercial Disposal Sites}

\begin{tabular}{l} 
Generator \\
Category \\
\hline
\end{tabular}

Government

Industrial

Medical

Utility

Total

\author{
Volume \\ $\left(\mathrm{ft}^{3}\right)$
}

8.50

$5,107.86$

$2,531.13$

$14,306.80$

$21,954.29$
Activity

(curies)

$$
24.85
$$

$1,094.20$

51.65

$59,781.38$

$60,952.08$
VOLUME PERCENTAGE BY SOURCE (Percentages $<.1 \%$ are not displayed)

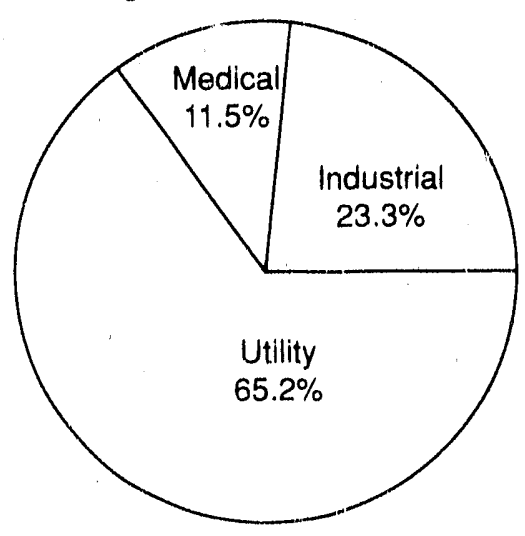

ACTIVITY PERCENTAGE BY SOURCE

(Percentages <.1\% are not displayed)

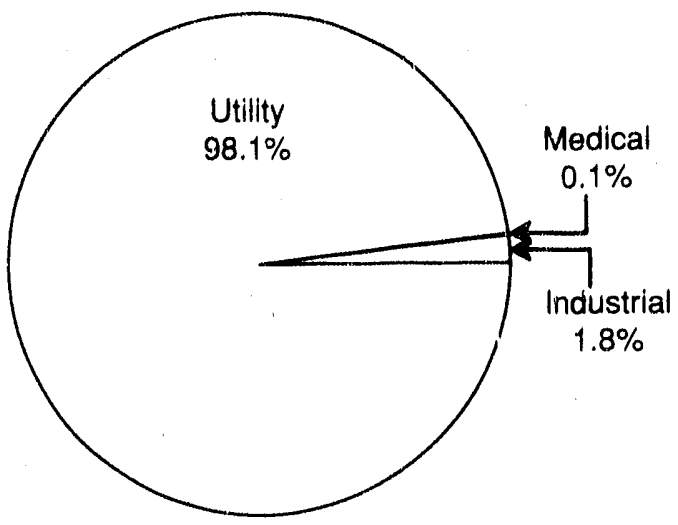




\section{MINNESOTA \\ Low-Level Radioactive Wastes Received at Commercial Disposal Sites}

\begin{tabular}{lrr}
\multicolumn{1}{c}{$\begin{array}{c}\text { Disposal } \\
\text { Site }\end{array}$} & $\begin{array}{c}\text { Volume } \\
\left(\mathrm{ft}^{3}\right)\end{array}$ & $\begin{array}{r}\text { Activity } \\
(\text { curies })\end{array}$ \\
Barnwell & $5,182.89$ & $\begin{array}{r}37,529.07 \\
1,131.48\end{array}$ \\
Beatty & 571.82 & $22,291.53$ \\
Richland & $16,199.58$ & $60,952.08$ \\
Total & $21,954.29$ &
\end{tabular}

VOLUME PERCENTAGE BY DISPOSAL SITE

(Percentages $<.1 \%$ are not displayed) Beatty

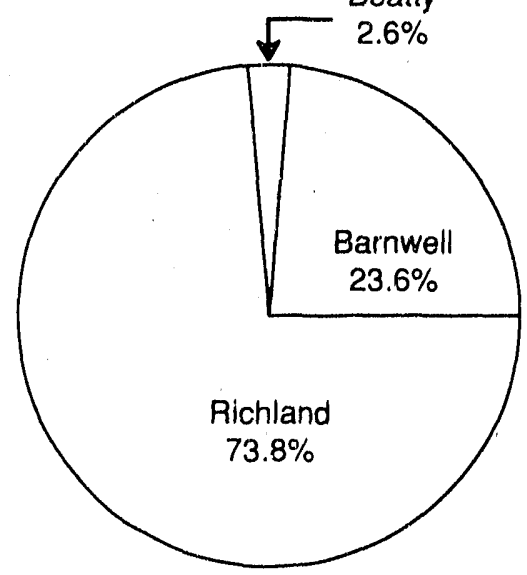

Direct Shipment

Waste Class

$$
\begin{aligned}
& \text { A } \\
& \text { B } \\
& \text { C }
\end{aligned}
$$

Total

Non-Direct Shipments

Grand Total

Volume $\left(\mathrm{ft}^{3}\right)$

$15,427.60$

278.60

161.10

$15,867.30$

$6,086.99$

$21,954.29$

Total Direct Shipment

Activity (curies)

$60,601.67$

Total Non-Direct Shipment

Activity (curies)

350.41

Grand Total (curies) $\frac{350.41}{60,952.08}$
ACTIVITY PERCENTAGE BY DISPOSAL SITE. (Percentages <. $1 \%$ are not displayed)

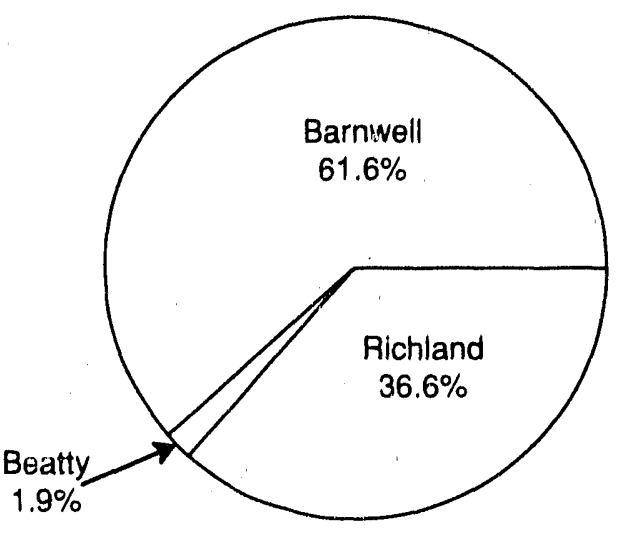

Volume Percentage by Waste Class for Direct Shipments and Total Non-Direct Volume Percentage (Percentages $<.1 \%$ are not displayed)

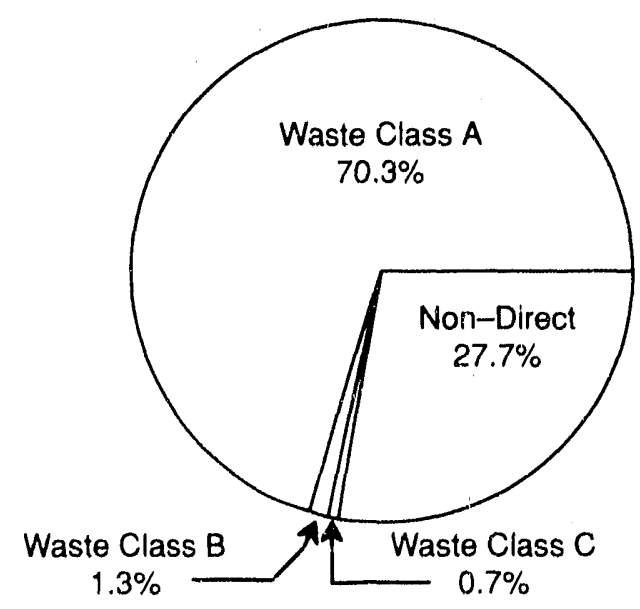




\section{MISSOURI \\ Low-Level Radioactive Wastes Received at Commercial Disposal Sites}

\begin{tabular}{l} 
Generator \\
Category \\
\hline
\end{tabular}

Academic

Government

Industrial

Medical

Utility

Total

$\begin{array}{r}\begin{array}{c}\text { Volume } \\ \left(\mathrm{ft}^{3}\right)\end{array} \\ \hline 3,868.16 \\ 7.00 \\ 7,492.65 \\ 66.83 \\ 7,368.00 \\ \hline\end{array}$

$18,802.64$
Activity

(curies)

91.00

20.32

79.94

1.32

600.45

793.03
VOLUME PERCENTAGE BY SOURCE (Percentages <. $1 \%$ are not displayed)

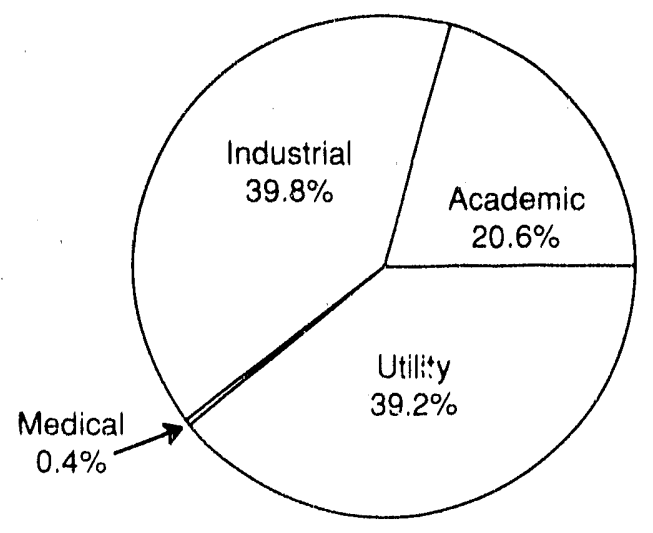

ACTIVITY PERCENTAGE BY SOURCE

(Percentages <. $1 \%$ are not displayed)

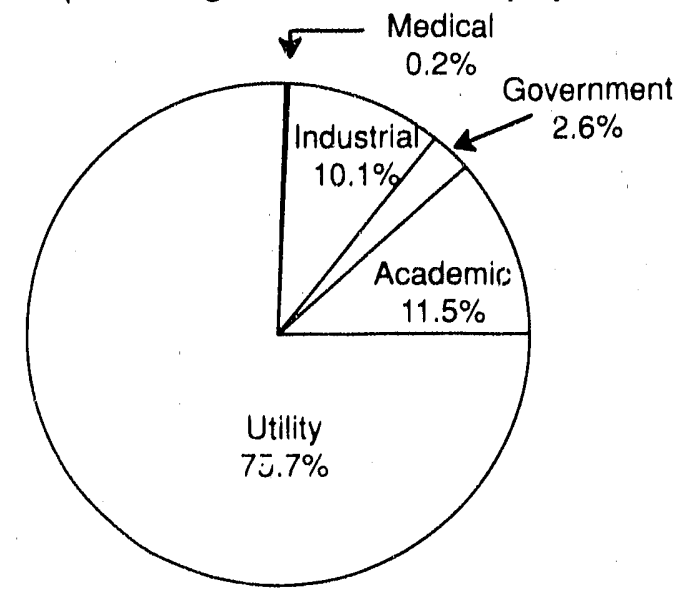




\section{MISSOURI \\ Low-Level Radioactive Wastes Received at Commercial Disposal Sites}

\begin{tabular}{lrr}
\multicolumn{1}{c}{$\begin{array}{c}\text { Disposal } \\
\text { Site }\end{array}$} & \multicolumn{1}{c}{$\begin{array}{c}\text { Volume } \\
\left(\mathrm{ft}^{3}{ }^{3}\right)\end{array}$} & \multicolumn{1}{c}{$\begin{array}{c}\text { Activity } \\
\text { (curies) }\end{array}$} \\
\cline { 1 - 1 } Barnwell & $8,282.00$ & 683.63 \\
Beatty & 469.72 & 7.21 \\
Richland & $10,050.92$ & 102.19 \\
Total & $18,802.64$ & 793.03
\end{tabular}

VOLUME PERCENTAGE BY DISPOSAL SITE (Percentages <.1\% are not displayed)

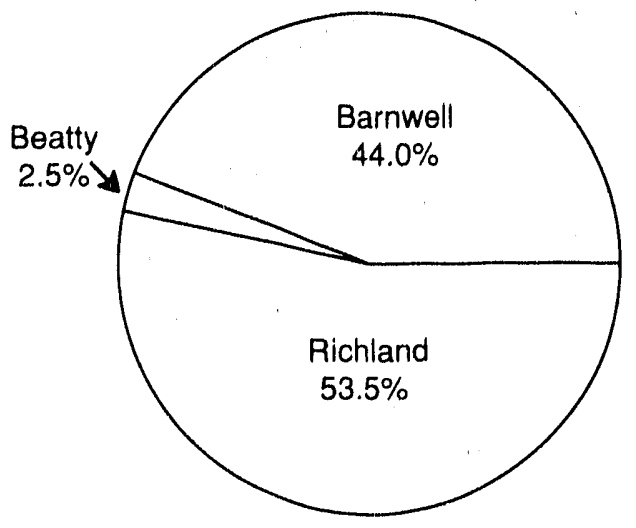

Direct Shipment

Waste Class

A

B

C

Total

Non-Direct Shipments

Grand Total

Total Direct Shipment

Activity (curies)

Total Non-Direct Shipment

Activity (curies)

Grand Total (curies)
ACTIVITY PERCENTAGE BY DISPOSAL SITE

(Percentages <. $1 \%$ are not displayed)

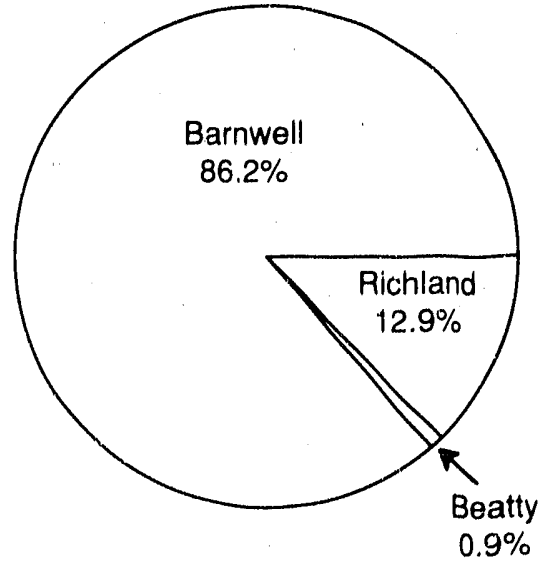

Volume Percentage by Waste Class for Direct Shipments and Total

Non-Direct Volume Percentage

(Percentages <. $1 \%$ are not displayed)

\begin{tabular}{c}
$\begin{array}{c}\text { Volume } \\
\left(\mathrm{ft}^{3}\right)\end{array}$ \\
\hline
\end{tabular}

$9,327.40$

715.90

83.40

$10,126.70$

$8,675.94$

$18,802.64$

677.90

Waste Class $C$

$0.4 \%$

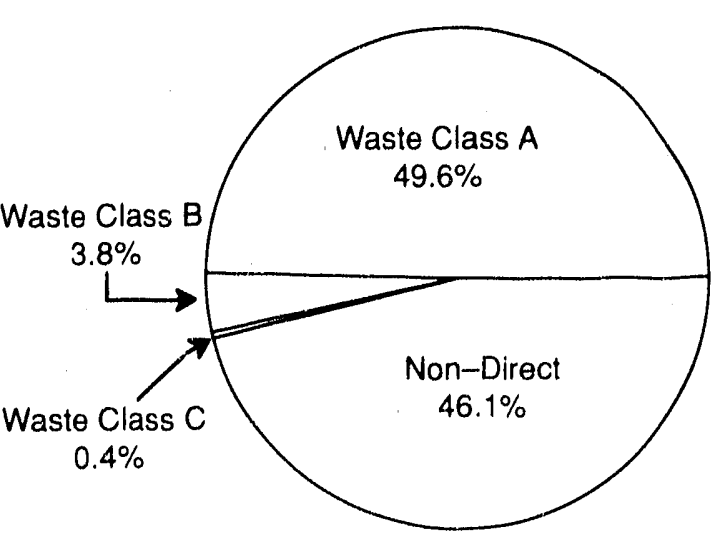

115.13

793.03 


\section{OHIO \\ Low-Level Radioactive Wastes Received at Commercial Disposal Sites}

\begin{tabular}{l} 
Generator \\
Category \\
\hline
\end{tabular}

Academic

Government

Industrial

Medical

Utility

Total

$\begin{array}{r}\begin{array}{c}\text { Volume } \\ \left(\mathrm{ft}^{3}\right)\end{array} \\ \hline 2,140.65 \\ 462.56 \\ 35,674.44 \\ 193.77 \\ 20,394.76 \\ \hline 58,866.18\end{array}$

Activity

(curies)

$\begin{array}{r}7.97 \\ 5.74 \\ 73.54 \\ 0.66 \\ 1,123.49 \\ \hline 1,211.40\end{array}$

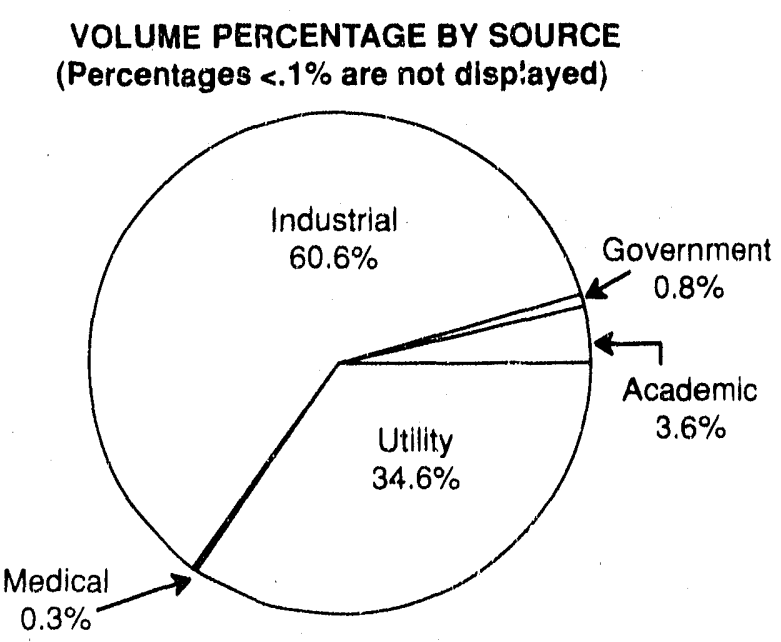

ACTIVITY PERCENTAGE BY SOURCE

(Percentages <. $1 \%$ are not displayed)

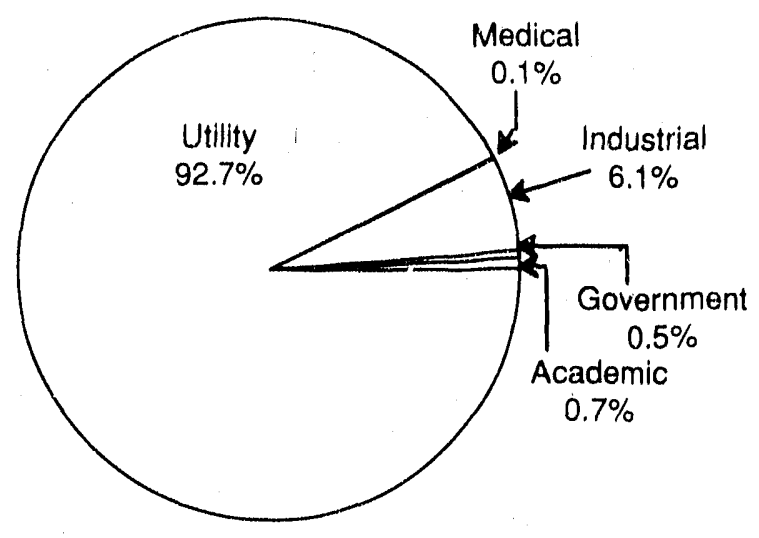




\section{OHIO \\ Low-Level Radioactive Wastes Received at Commercial Disposal Sites}

\begin{tabular}{lr}
\multicolumn{1}{c}{$\begin{array}{c}\text { Disposal } \\
\text { Site }\end{array}$} & \multicolumn{1}{c}{$\begin{array}{c}\text { Volume } \\
\left(\mathrm{ft}^{3}\right)\end{array}$} \\
Barnwell & $\begin{array}{r}4,583.60 \\
\text { Beatty }\end{array}$ \\
Richland & $1,052.45$ \\
Total & $5,230.13$ \\
\hline
\end{tabular}

Activity

(curies)

Barnwell

$42,583.60$

725.05

35.07

451.28

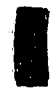

VOLUME PERCENTAGE BY DISPOSAL SITE

(Percentages <. $1 \%$ are not displayed)

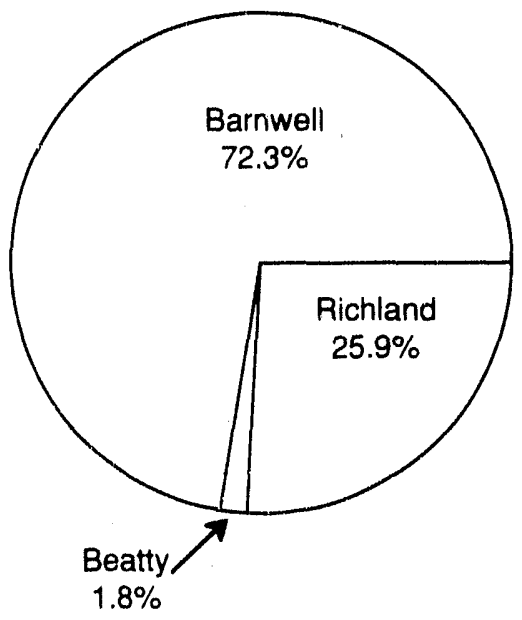

ACTIVITY PERCENTAGE BY DISPOSAL SITE

(Percentages <.1\% are not displayed)

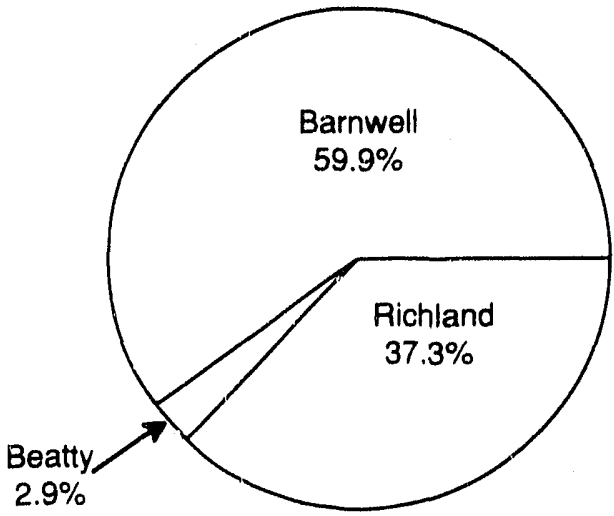

Volume Percentage by Waste Class for Direct Shipments and Total Non-Direct Volume Percentage (Percentages <.1\% áre not displayed)
Direct Shipment

Waste Class

A

B

C

Total

Non-Direct Shipments

Grand Total

Total Direct Shipment

Activity (curies)

Total Non-Direct Shipment

Activity (curies)

Grand Total (curies)
Volume

$\left(\mathrm{ft}^{3}\right)$

$49,482.40$

199.80

139.94

$49,822.14$

$9,044.04$

$58,866.18$

$1,153.17$

58.23

1.211 .40

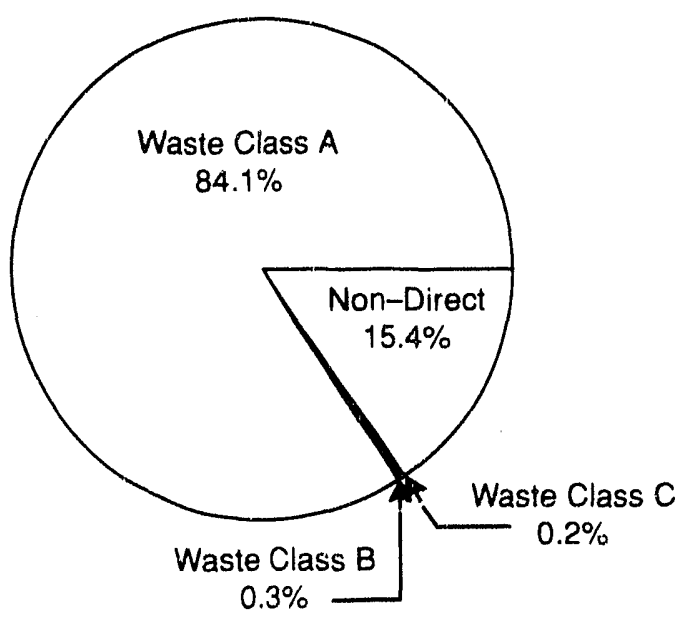




\section{WISCONSIN \\ Low-Level Radioactive Wastes Received at Commercial Disposal Sites}

\author{
Generator \\ Category
}

Academic

Government

Industrial

Medical

Utility

Total

$\begin{array}{r}\begin{array}{c}\text { Volume } \\ \left(\mathrm{ft}^{3}\right)\end{array} \\ \hline \\ 191.09 \\ 3.85 \\ 154.58 \\ 65.76 \\ 6,465.65 \\ \hline 6.880 .93\end{array}$

Activity

(curies)

12.82

0.83

0.56

0.23

1.060 .45

$1,074.88$
VOLUME PERCENTAGE BY SOURCE

(Percentages <. $1 \%$ are not displayed)
ACTIVITY PERCENTAGE BY SOURCE

(Percentages <. $1 \%$ are not displayed)
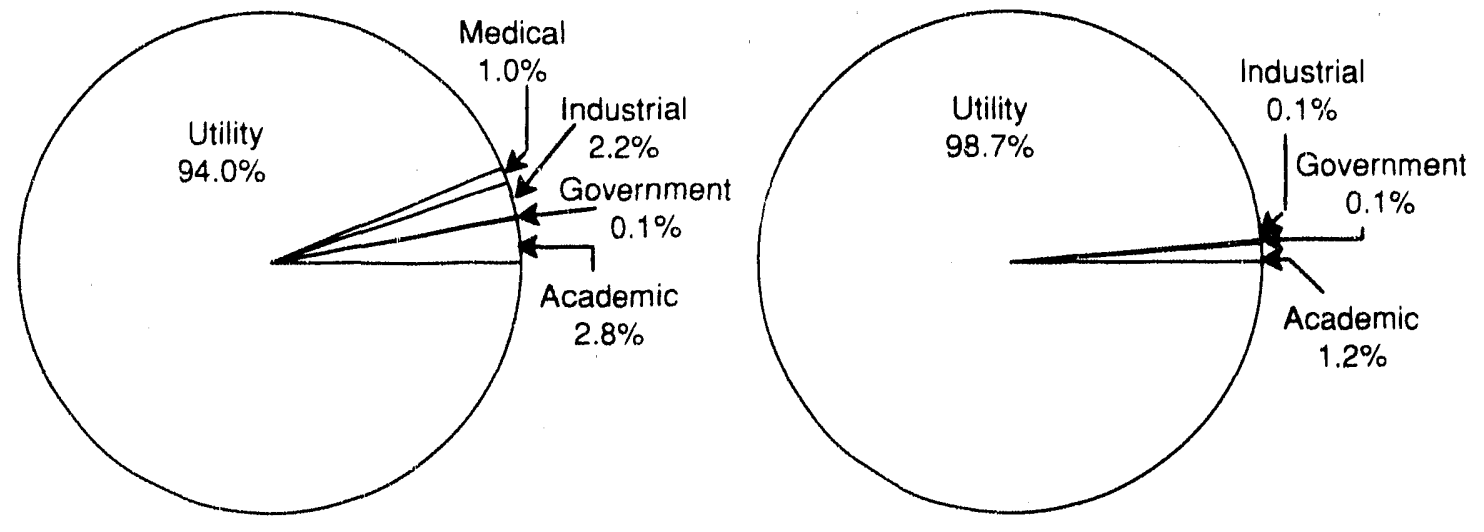


\section{WISCONSIN \\ Low-Level Radioactive Wastes Received at Commercial Disposal Sites}

\begin{tabular}{c} 
Disposal \\
Site \\
\hline
\end{tabular}

Barnwell

Beatty

Richland

Total

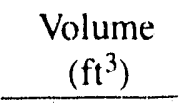

$$
6,465.75
$$

67.89

347.29

$6,880.93$
Activity

(curies)

$1,061.28$

6.12

7.49

$1,074.88$
VOLUME PERCENTAGE BY DISPOSAL SITE

(Percentages $<.1 \%$ are not displayed)

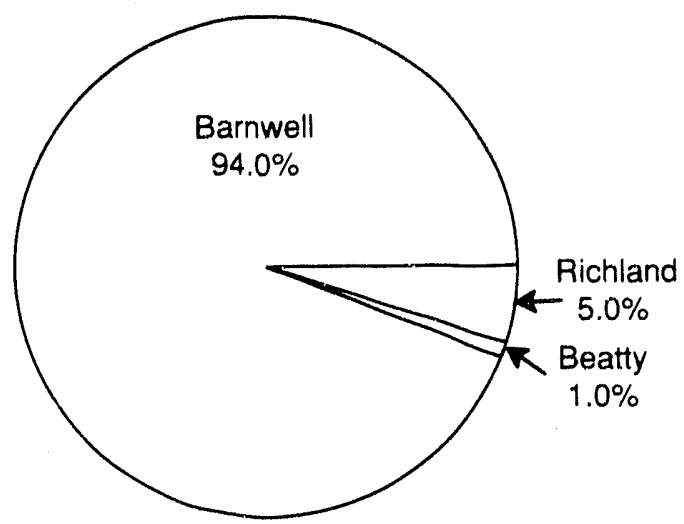

ACTIVITY PERCENTAGE BY DISPOSAL SITE

(Percentages <. $1 \%$ are not displayed)

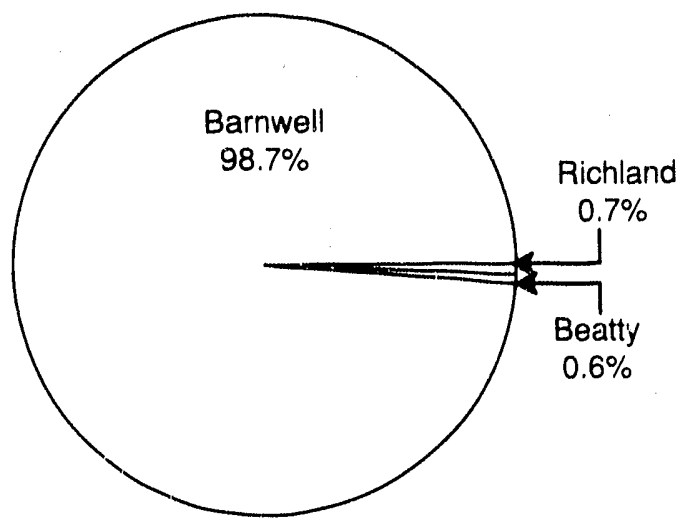

Volume Percentage by Waste Class for Direct Shipments and Total Non-Direct Volume Percentage (Percentages <. $1 \%$ are not displayed)
Direct Shipment

Waste Class

A

B

C

Total

Grand Total

Total Direct Shipment

Activity (curies)

Total Non-Direct Shipment

Activity (curies)

Grand Total (curies)
Non-Direct Shipments
Volume

$\left(\mathrm{ft}^{3}\right)$

$2,056.80$

920.90

125.20

$3,102.90$

$3,778.03$

$6,880.93$

$1,058.69$

16.19

$1,074.88$ 


\section{NORTHEAST COMPACT Low-Level Radioactive Wastes Received at Commercial Disposal Sites}

\begin{tabular}{|c|c|c|}
\hline States & $\begin{array}{c}\text { Volume } \\
\left(\mathrm{ft}^{3}\right)\end{array}$ & $\begin{array}{l}\text { Activity } \\
\text { (curies) }\end{array}$ \\
\hline Connecticut & $49,078.29$ & $21,884.63$ \\
\hline New Jersey & $53,539.95$ & $317,832.66$ \\
\hline Total & $102,618.24$ & $339,717.29$ \\
\hline
\end{tabular}

VOLUME PERCENTAGE BY STATE

(Percentages <. $1 \%$ are not displayed)

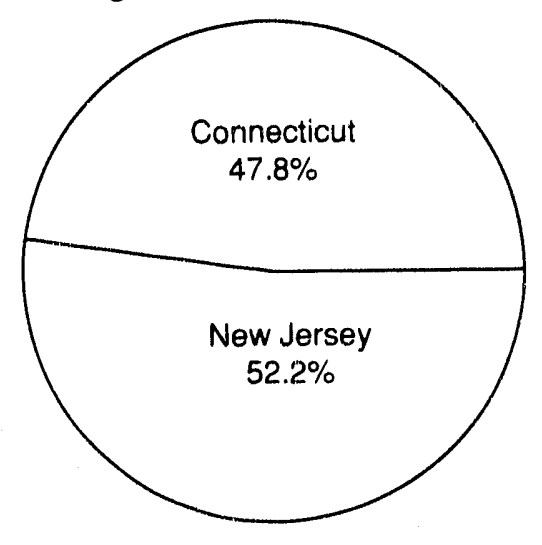

VOLUME PERCENTAGE BY CATEGORY

(Percentages <. $1 \%$ are not displayed)

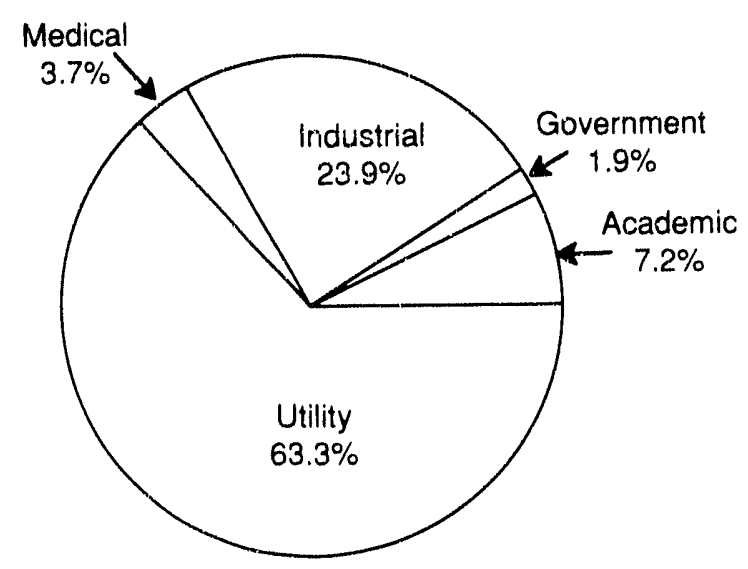




\section{CONNECTICUT \\ Low-Level Radioactive Wastes Received at Commercial Disposal Sites}

\begin{tabular}{l} 
Generator \\
Category \\
\hline
\end{tabular}

Academic

Government

Industrial

Medical

Utility

Total

$\begin{array}{r}\begin{array}{c}\text { Volume } \\ \left(\mathrm{ft}^{3}\right)\end{array} \\ \hline 2,035.87 \\ 1,352.90 \\ 8,776.19 \\ 252.59 \\ 36,660.74 \\ \hline 49,078.29\end{array}$

$49,078.29$
Activity

(curies)

$\begin{array}{r}5.34 \\ 0.24 \\ 9.62 \\ 0.78 \\ 21,868.65 \\ \hline 21,884.63\end{array}$

VOLUME PERCENTAGE BY SOURCE

(Percentages $<.1 \%$ are not displayed)

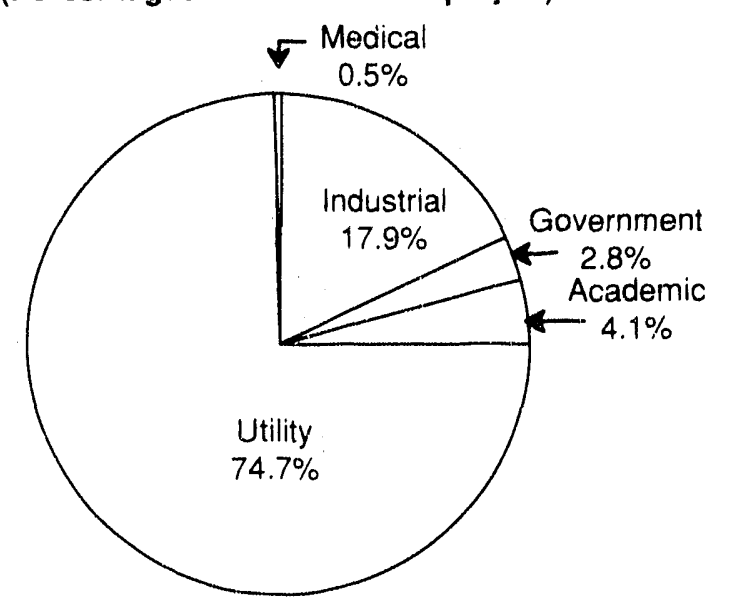

ACTIVITY PERCENTAGE BY SOURCE

(Percentages $<.1 \%$ are not displayed)

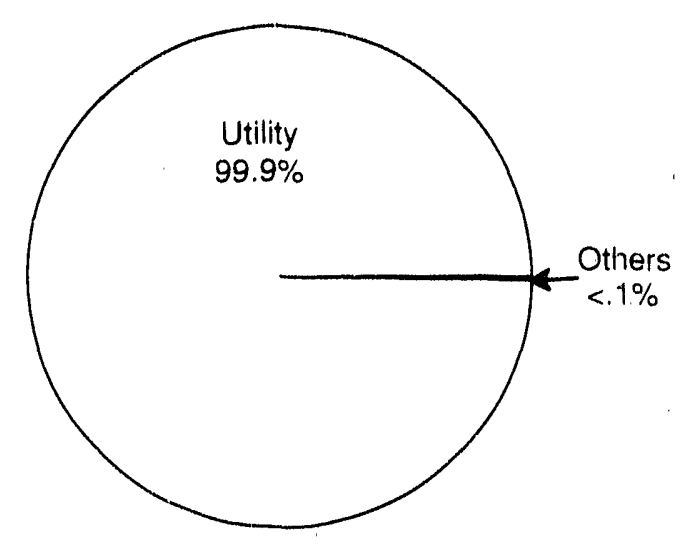




\section{CONNECTICUT \\ Low-Level Radioactive Wastes Received at Commercial Disposal Sites}

\begin{tabular}{c} 
Disposal \\
Site \\
\hline
\end{tabular}

Barnwell

Beatty

Richland

Total

\author{
Volume \\ $\left(\mathrm{ft}^{3}\right)$
}

$44,089.41$

130.93

$4,857.95$

$49,078,29$
Activity

(curies)

$21,869.87$

0.75

14.01

$21,884.63$
VOLUME PERCENTAGE BY DISPOSAL SITE

(Percentages <.1\% are not displayed)

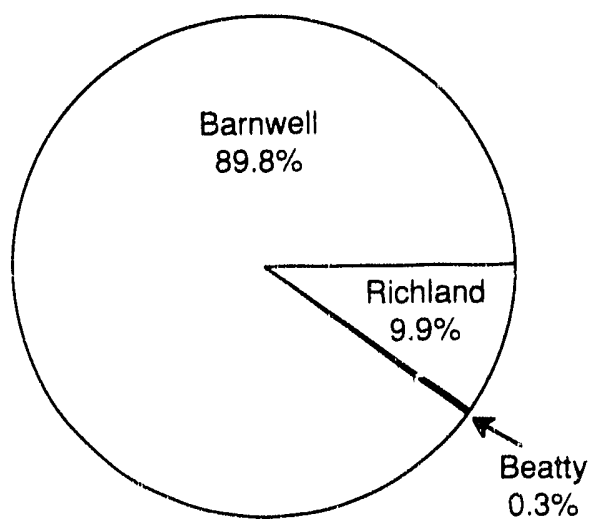

Direct Shipment

Waste Class

A

B

C

Total

Non-Direct Shipments

Grand Total

Total Direct Shipment

Activity (curies)

Total Non-Direct Shipment

Activity (curies)

Grand Total (curies)
Volume

$$
\left(\mathrm{ft}^{3}\right)
$$

$33,931.20$

$1,655.40$

538.70

$36,125.30$

$12,952.99$

$49,078.29$

$21,865.91$

18.72

$21,884.63$
ACTIVITY PERCENTAGE BY DISPOSAL SITE (Percentages $4.1 \%$ are not displayed)

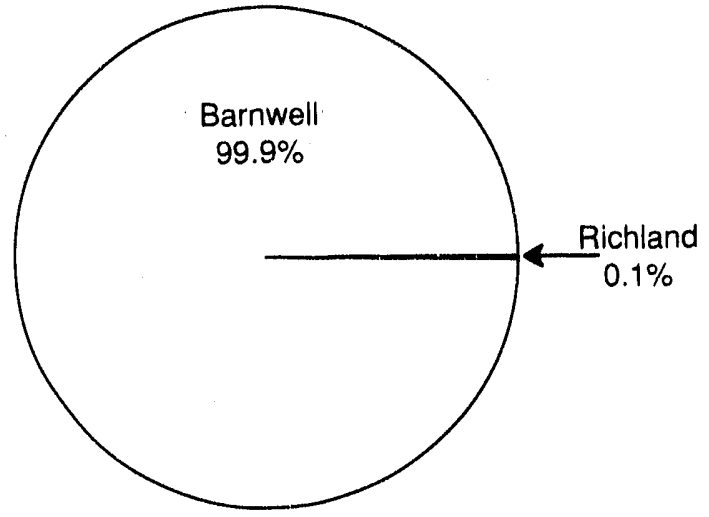

Volume Percentage by Waste Class for Direct Shipments and Total Non-Direct Volume Percentage (Percentages $<.1 \%$ are not displayed)

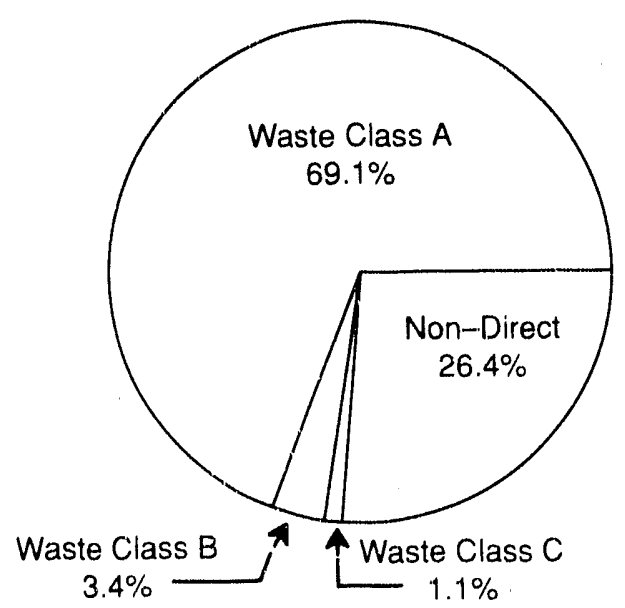

Waste Class B

$1.1 \%$ 


\section{NEW JERSEY \\ Low-Level Radioactive Wastes Received at Commercial Disposal Sites}

\begin{tabular}{l} 
Generator \\
Category \\
\hline
\end{tabular}

Academic

Government

Industrial

Medical

Utility

Total

$$
\begin{array}{r}
\begin{array}{c}
\text { Volume } \\
\left(\mathrm{ft}^{3}\right)
\end{array} \\
\hline 5,356.53 \\
562.51 \\
15,774.01 \\
3,585.26 \\
28,261.64 \\
\hline 53,539.95
\end{array}
$$

Activity

(curies)

9.84

$1,401.84$

$26,273.53$

5.07

$290,142.38$

$317,832.66$

VOLUME PERCENTAGE BY SOURCE

ACTIVITY PERCENTAGE BY SOURCE

(Percentages <. $1 \%$ are not displayed)

(Percentages <. $1 \%$ are not displayed)
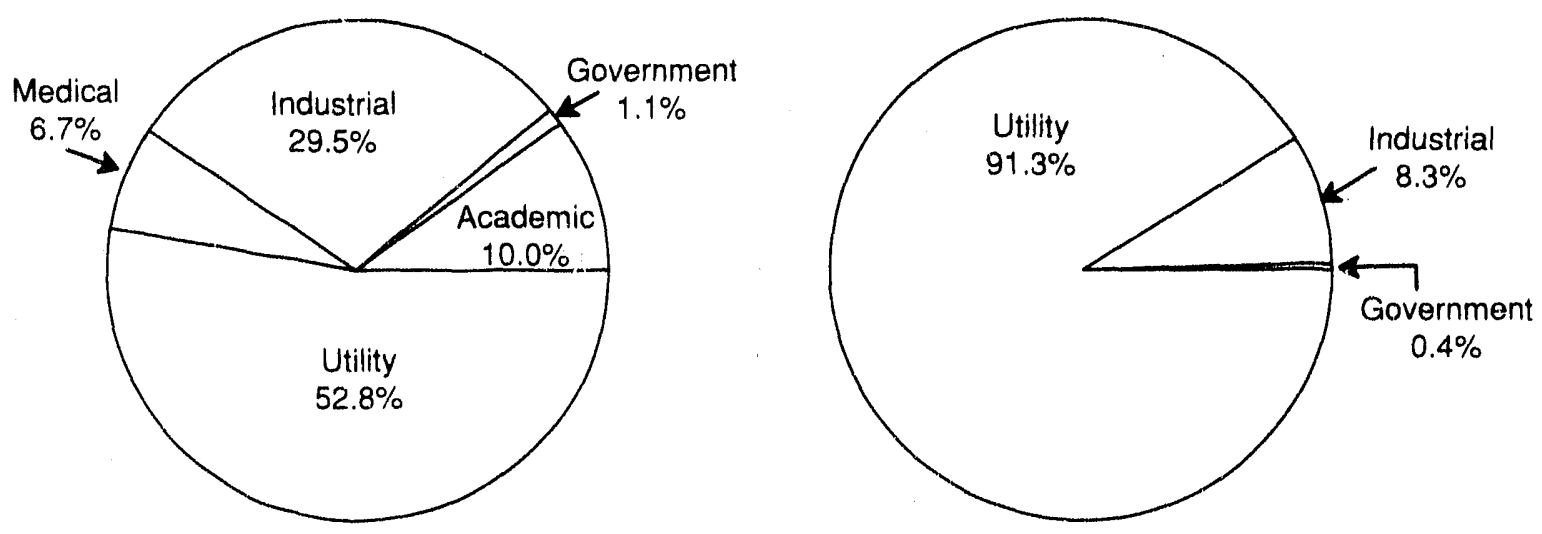


\section{NEW JERSEY \\ Low-Level Radioactive Wastes Received at Commercial Disposal Sites}

\begin{tabular}{|c|c|c|}
\hline $\begin{array}{l}\text { Disposal } \\
\text { Site }\end{array}$ & $\begin{array}{c}\text { Volume } \\
\left(\mathrm{ft}^{3}\right)\end{array}$ & $\begin{array}{l}\text { Activity } \\
\text { (curies) }\end{array}$ \\
\hline Barnwell & $41,159.25$ & $317,684.12$ \\
\hline Beatty & $2,518.35$ & 30.44 \\
\hline Richland & $9,862.35$ & 118.10 \\
\hline Total & $53,539.95$ & $317,832.66$ \\
\hline
\end{tabular}

(Percentages <.1\% are not displayed)

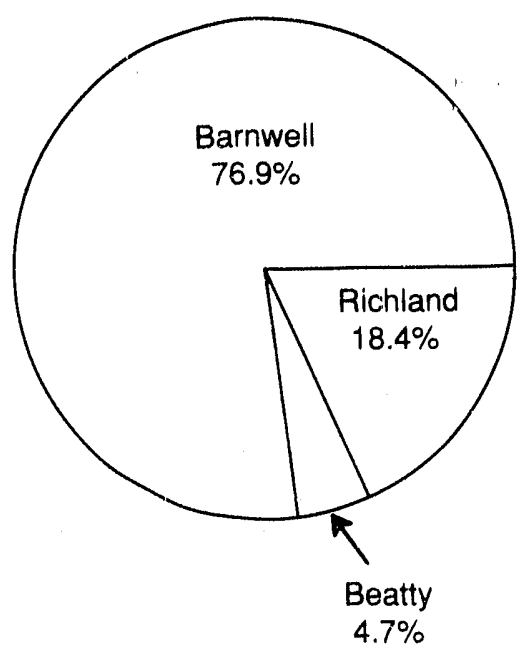

VOLUME PERCENTAGE BY DISPOSAL. SITE

ACTIVITY PERCENTAGE BY DISPOSAL SITE

(Percentages $<.1 \%$ are not displayed)

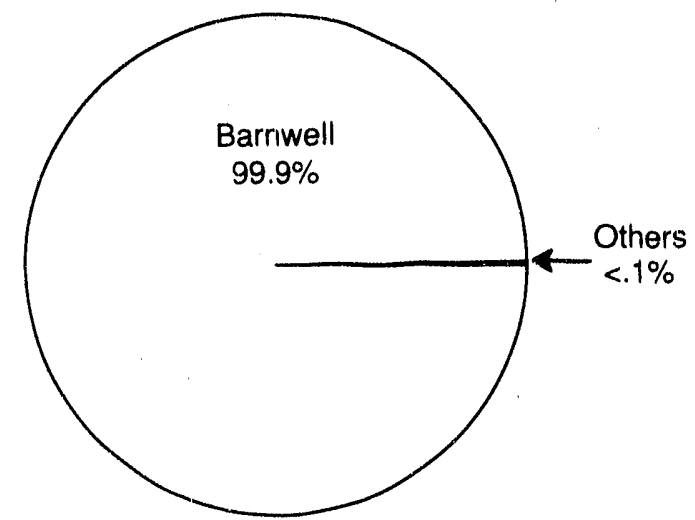

Volume Percentage by Waste Class for Direct Shipments and Total Non-Direct Volume Percentage (Percentages <. $1 \%$ are not displayed)

Direct Shipment

Waste Class

$$
\begin{aligned}
& \text { A } \\
& \text { B } \\
& \text { C }
\end{aligned}
$$

Total

Non-Direct Shipments

Grand Total

Total Direct Shipment

Activity (curies)

Total Non-Direct Shipment

Activity (curies)

Grand Total (curies)

$$
\begin{array}{r}
\begin{array}{c}
\text { Volume } \\
\left(\mathrm{ft}^{3}\right)
\end{array} \\
\hline \begin{array}{r}
15,390.60 \\
3,566.30 \\
911.30
\end{array} \\
\hline \begin{array}{r}
19,868.20 \\
33,671.75
\end{array} \\
\hline 53,539.95 \\
316,646.25 \\
1,186.41 \\
\hline 317,832.66
\end{array}
$$

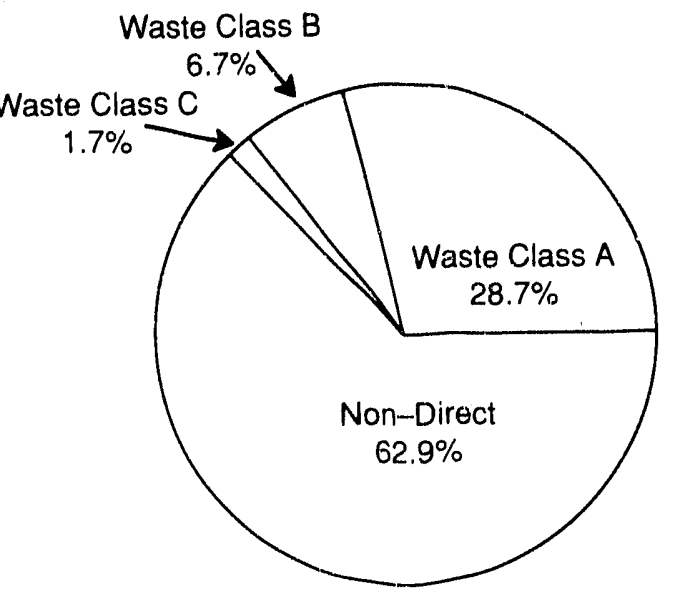




\section{NORTHWEST COMPACT \\ Low-Level Radioactive Wastes Received at Commercial Disposal Sites}

\begin{tabular}{lrr}
\multicolumn{1}{c}{ States } & \multicolumn{1}{c}{$\begin{array}{c}\text { Volume } \\
\left(\mathrm{ft}^{3}\right)\end{array}$} & \multicolumn{1}{c}{$\begin{array}{c}\text { Activity } \\
\text { (curies) }\end{array}$} \\
Alaska & 10.59 & 3.90 \\
Hawaii & $6,203.82$ & 6.51 \\
Idaho & 137.99 & 1.70 \\
Montana & 107.01 & 1.06 \\
Oregon & $75,907.48$ & 465.75 \\
Utah & $6,299.31$ & 7.88 \\
Washington & $26,408.54$ & $1,105.04$ \\
Total & & $1,591.82$
\end{tabular}

VOLUME PERCENTAGE BY STATE

(Percentages <.1\% are not displayed)

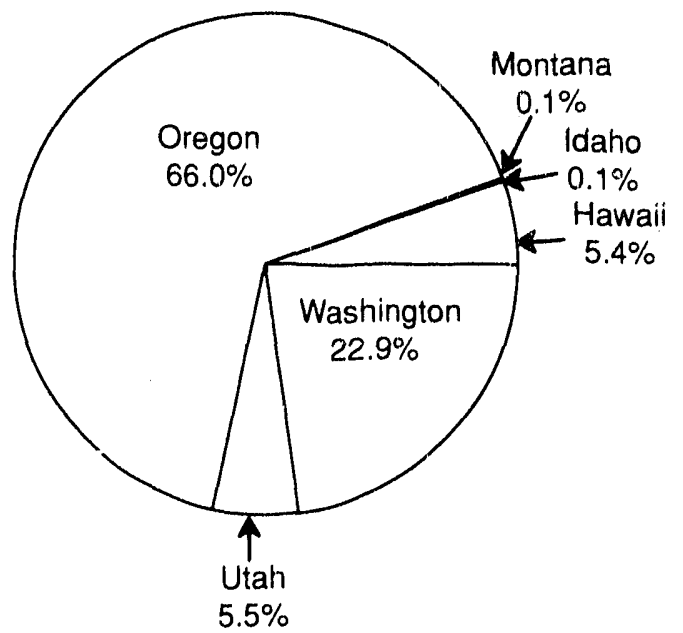

VOL.UME PERCENTAGE BY CATEGORY

(Percentages $<.1 \%$ are not displayed)

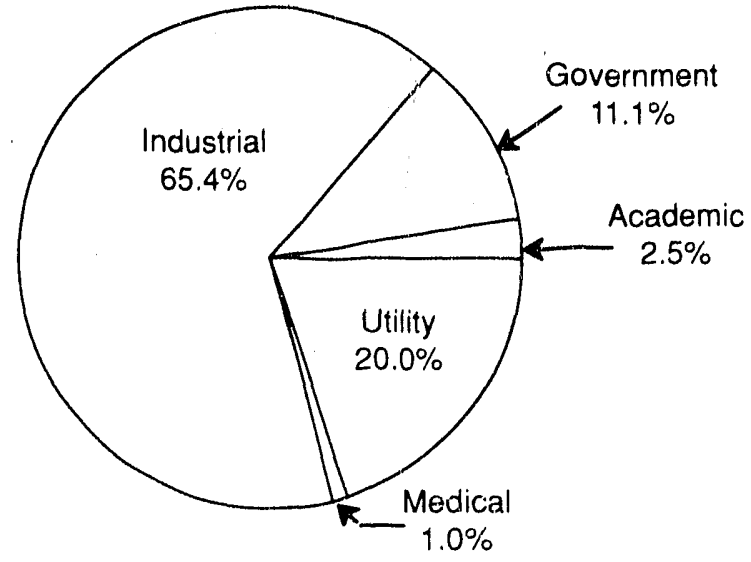




\section{ALASKA \\ Low-Level Radioactive Wastes Received at Commercial Disposal Sites}

\begin{tabular}{l} 
Generator \\
Category \\
\hline
\end{tabular}

Academic

Government

Medical

Total

\section{Volume}

$\left(\mathrm{ft}^{3}\right)$

$\begin{array}{r}0.54 \\ 9.51 \\ 0.54 \\ \hline\end{array}$

10.59
Activity

(curies)

\begin{tabular}{c}
$<1$ \\
3.90 \\
$<1$ \\
\hline
\end{tabular}

VOLUME PERCENTAGE BY SOURCE (Percentages <.1\% are not displayed)

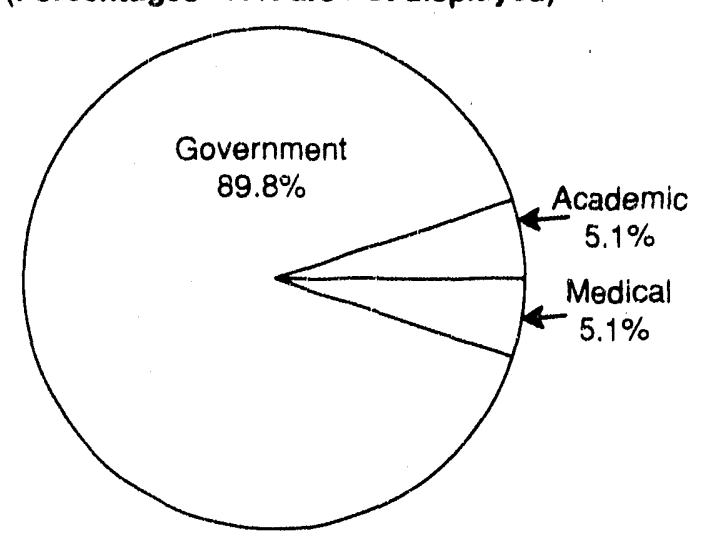

ACTIVITY PERCENTAGE BY SOURCE

(Percentages <. $1 \%$ are not displayed)

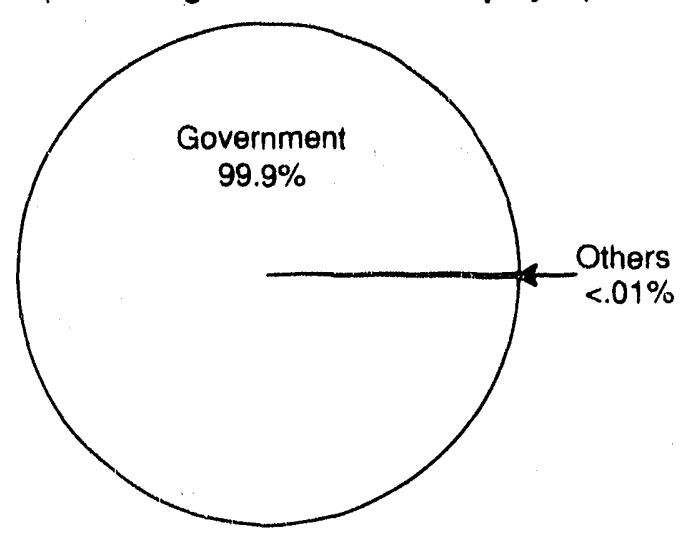




\section{ALASKA \\ Low-Level Radioactive Wastes Received at Commercial Disposal Sites}

Disposal
Site

Beatty

Richland

Total
Volume

$\left(\mathrm{ft}^{3}\right)$

4.55

6.04

10.59
Activity

(curies)

0.01

3.89

3.90
VOLUME PERCENTAGE BY DISPOSAL SITE

(Percentages <. $1 \%$ are not displayed)

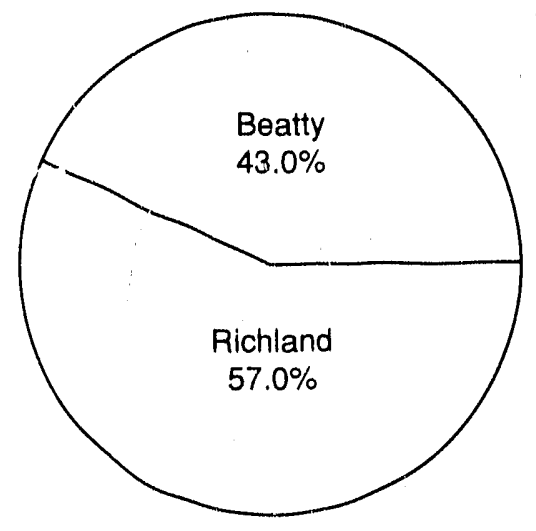

ACTIVITY PERCENTAGE BY DISPOSAL SITE

(Percentages <. $1 \%$ are not displayed)

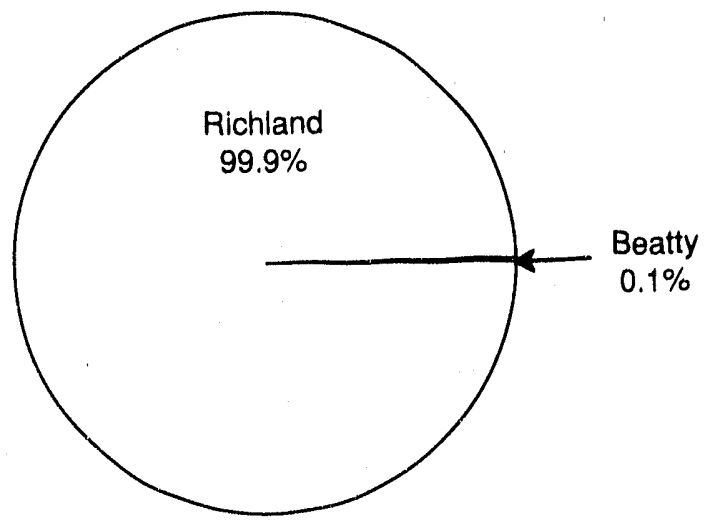

Volume Percentage by Waste Class for Direct Shipments and Total

Non-Direct Volume Percentage

(Percentages <.1\% are not displayed)

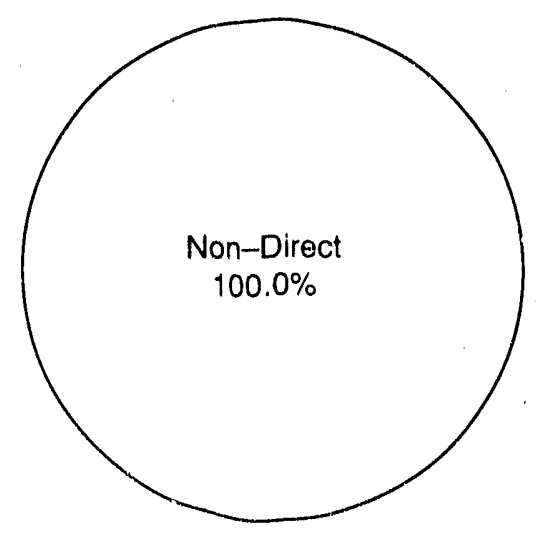




\section{HAWAII \\ Low-Level Radioactive Wastes Received at Commercial Disposal Sites}

\section{Generator \\ Category}

Academic

Government

Industrial

Total

$\begin{array}{r}\begin{array}{c}\text { Volume } \\ \left(\mathrm{ft}^{3}\right)\end{array} \\ \hline 262.50 \\ 5,933.82 \\ 7.50 \\ \hline\end{array}$

$6,203.82$
Activity

(curies)

5.17

0.88

0.45

6.51
VOLUME PERCENTAGE BY SOURCE (Percentages <.1\% are not displayed)

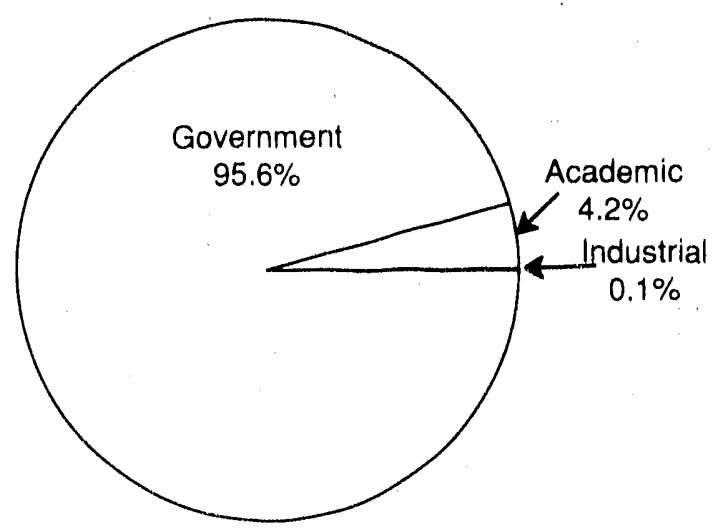

ACTIVITY PERCENTAGE BY SOURCE (Percentages <. $1 \%$ are not displayed)

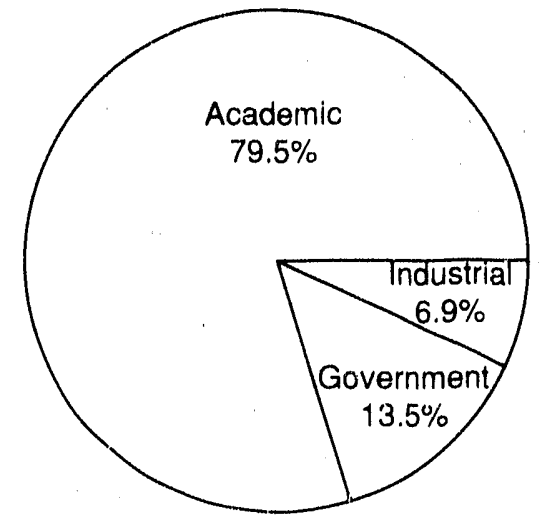




\section{HAWAII \\ Low-Level Radioactive Wastes Received at Commercial Disposal Sites}

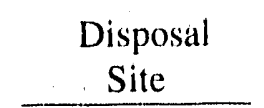

Beatty

Richland

Total

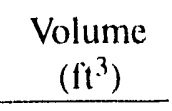

7.50

$6,196.32$

$6,203.82$
Activity

(curies)

0.45

6.06

6.51
VOLUME PERCENTAGE BY DISPOSAL SITE

(Percentages $<.1 \%$ are not displayed)

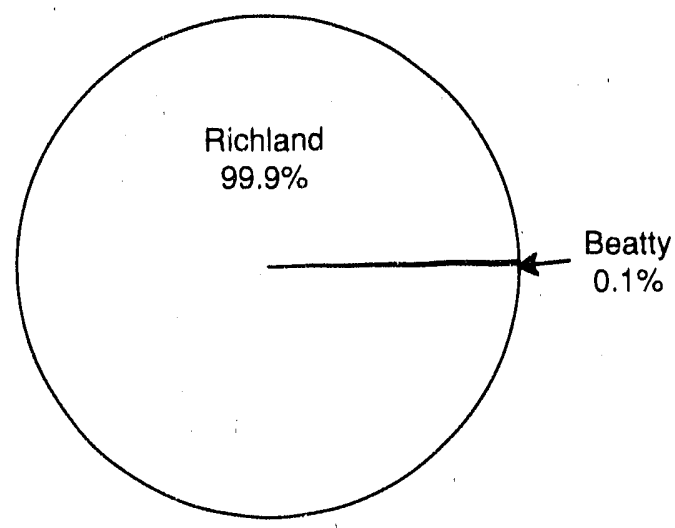

ACTIVITY PERCENTAGE BY DISPOSAL SITE

(Percentages <. $1 \%$ are not displayed)

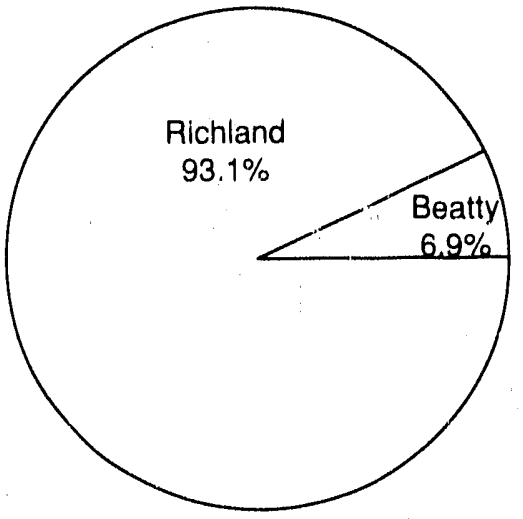

Volume Percentage by Waste Class

for Direct Shipments and Total

Non-Direct Volume Percentage

(Percentages $<.1 \%$ are not displayed)
Direct Shipment

Waste Class

A

B

C

Total

Non-Direct Shipments

Grand Total

Total Direct Shipment

Activity (curies)

Total Non-Direct Shipment

Activity (curies)

Grand Total (curies)
Volume

$\left(\mathrm{ft}^{3}\right)$

$5,933.82$

0.00

0.00

$5,933.82$

270.00

$6,203.82$

0.88

5.62

6.51

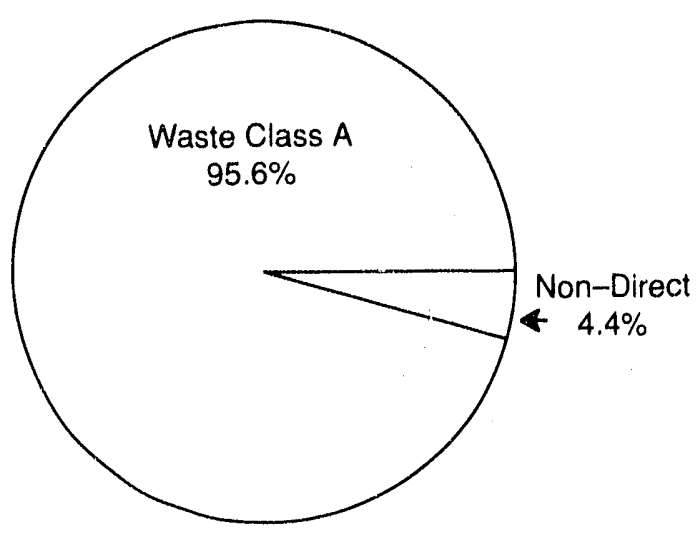




\section{IDAHO \\ Low-Level Radioactive Wastes Received at Commercial Disposal Sites}

\begin{tabular}{lrr}
$\begin{array}{l}\text { Generator } \\
\text { Category }\end{array}$ & $\begin{array}{c}\text { Volume } \\
\left(\mathrm{ft}^{3}\right)\end{array}$ & $\begin{array}{r}\text { Activity } \\
\text { (curies) }\end{array}$ \\
\cline { 2 - 2 } $\begin{array}{l}\text { Government } \\
\text { Industrial }\end{array}$ & $\begin{array}{r}5.00 \\
132.99\end{array}$ \\
Total & 137.99 & 1.31 \\
\cline { 2 - 3 } & & 0.39 \\
\hline
\end{tabular}

VOLUME PERCENTAGE BY SOURCE

ACTIVITY PERCENTAGE BY SOURCE

(Percentages <. $1 \%$ are not displayed)

(Percentages <. $1 \%$ are not displayed)
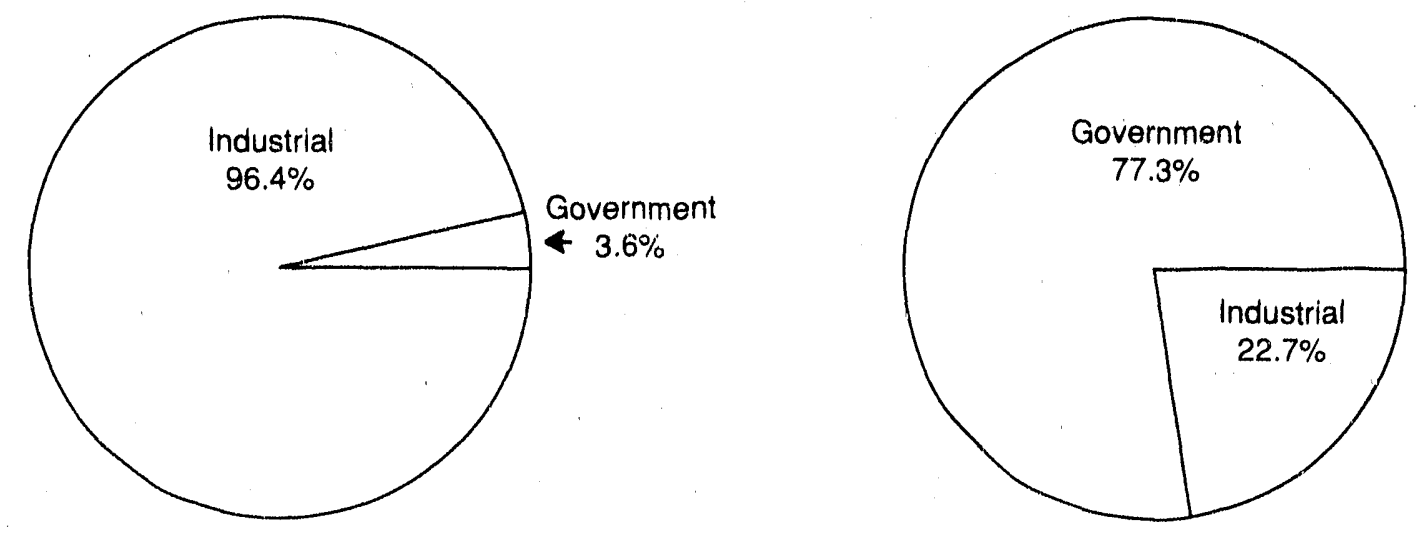


\section{IDAHO \\ Low-Level Radioactive Wastes Received at Commercial Disposal Sites}

\begin{tabular}{lrr}
\multicolumn{1}{c}{$\begin{array}{c}\text { Disposal } \\
\text { Site }\end{array}$} & $\begin{array}{c}\text { Volume } \\
\left(\mathrm{ft}^{3}\right)\end{array}$ & $\begin{array}{r}\text { Activity } \\
\text { (curies) }\end{array}$ \\
Beatty & $\begin{array}{r}30.00 \\
107.99\end{array}$ & 0.33 \\
Richland & 137.99 & 1.36 \\
Total & & 1.70
\end{tabular}

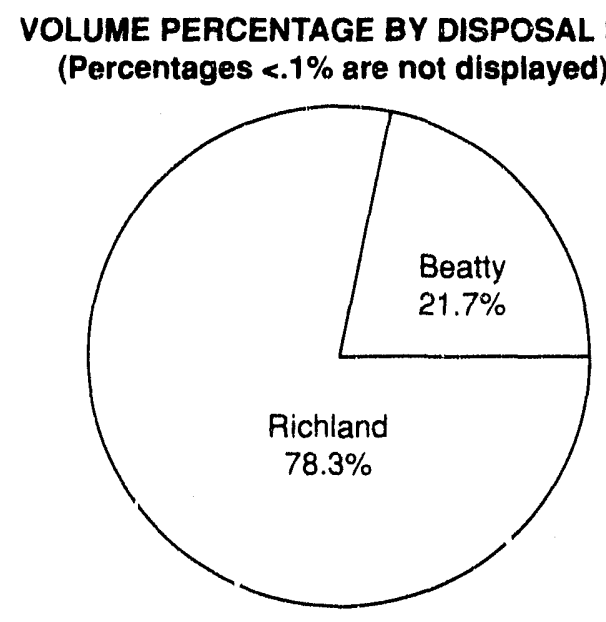
ACTIVITY PERCENTAGE BY DISPOSAL SITE
(Percentages <. $1 \%$ are not displayed)

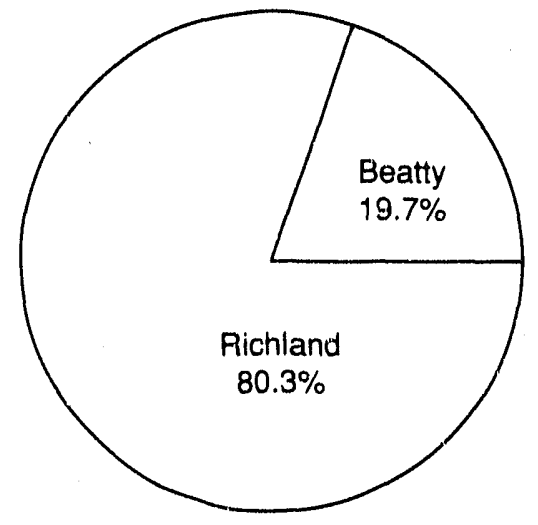

Volume Percentage by Waste Class

for Direct Shipments and Total

Non-Direct Volume Percentage

(Percentages <. $1 \%$ are not displayed)

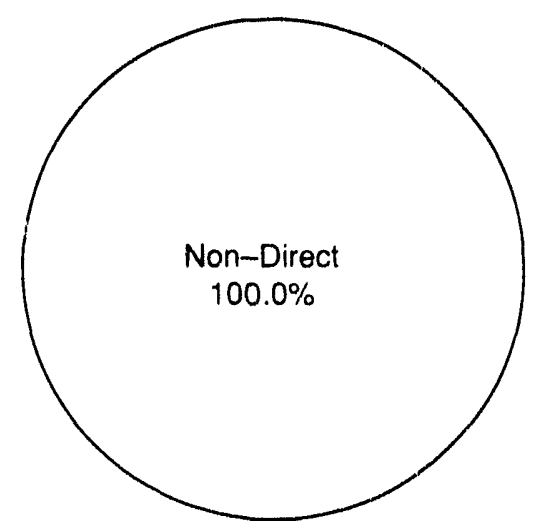




\section{MONTANA \\ Low-Level Radioactive Wastes Received at Commercial Disposal Sites}

\begin{tabular}{l} 
Generator \\
Category \\
\hline
\end{tabular}

Academic

Government

Industrial

Total

$\begin{array}{r}\begin{array}{c}\text { Volume } \\ \left(\mathrm{ft}^{3}\right)\end{array} \\ \hline 37.50 \\ 0.30 \\ 69.21 \\ \hline\end{array}$

107.01
Activity

(curies)

$\begin{array}{r}0.50 \\ <1 \\ 0.55 \\ \hline \\ \hline\end{array}$

VOLUME PERCENTAGE BY SOURCE (Percentages $<.1 \%$ are iiot displayed)

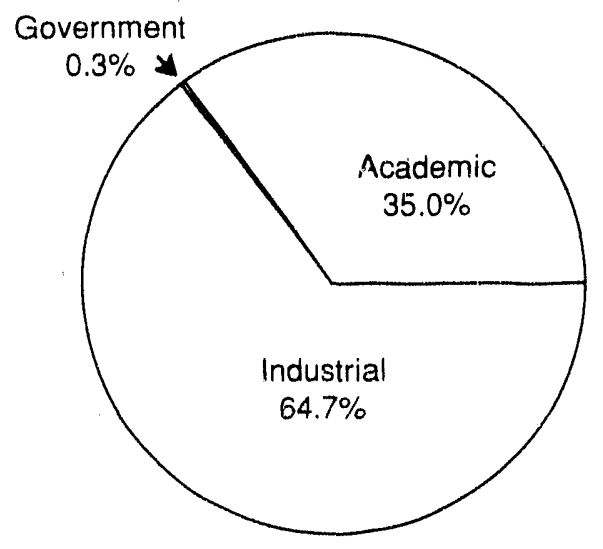

ACTIVITY PERCENTAGE BY SCURCE

(Percentages $4.1 \%$ are not displayed)

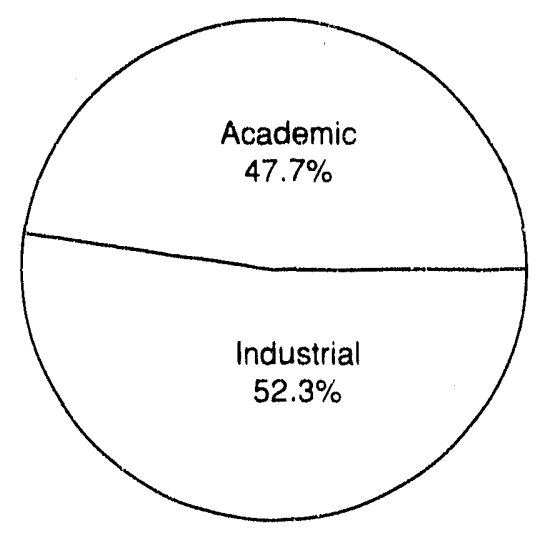




\section{MONTANA \\ Low-Level Radioactive Wastes Received at Commercial Disposal Sites}

Disposal
Site

Beatty

Richland

Total

\section{Volume}

$\left(f t^{3}\right)$

5.92

101.09

107.01
Activity

(curies)

0.51

0.55

1.06
VOLUME PERCENTAGE BY DISPOSAL SITE

(Percentages <. $1 \%$ are not displayed)

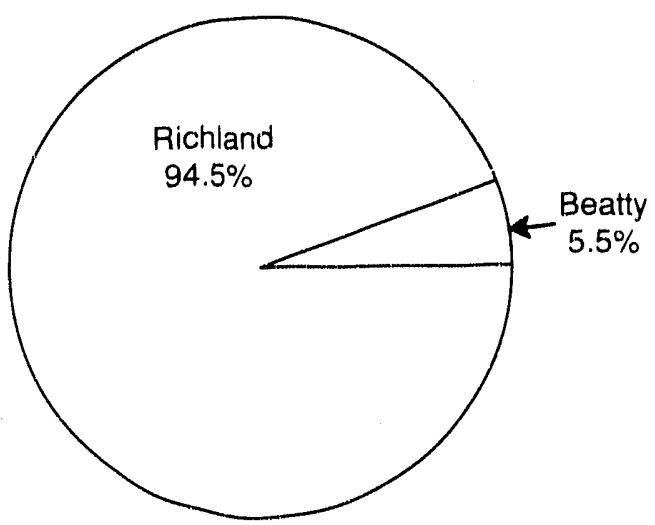

ACTIVITY PERCENTAGE BY DISPOSAL SITE (Percentages <. $1 \%$ are not displayed!

Volume Percentage by Waste Class for Direct Shipments and Total Non-Direct Volume Percentage (Percentages <. $1 \%$ are not displayed)

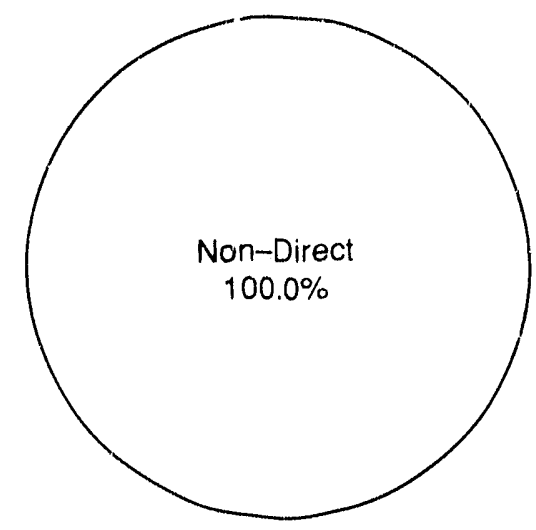




\section{OREGON \\ Low-Level Radioactive Wastes Received at Commercial Disposal Sites}

\begin{tabular}{l} 
Generator \\
Category \\
\hline
\end{tabular}

Academic

Industrial

Medical

Utility

Total
Volume

$\left(\mathrm{ft}^{3}\right)$

510.95

$65,235.52$

11.51

$10,149.50$

$75,907.48$
Activity

(curies)

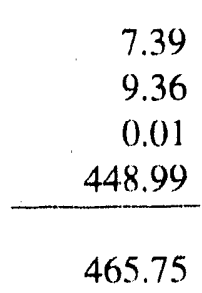

VOLUME PERCENTAGE BY SOURCE (Percentages <. $1 \%$ are not displayed)

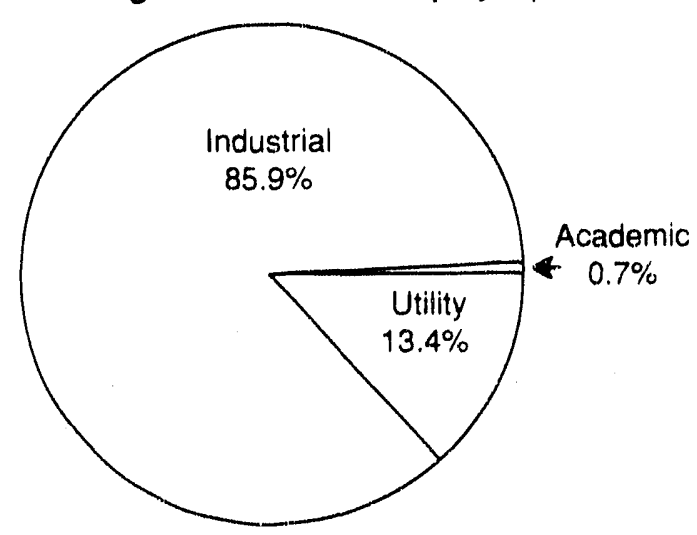

ACTIVITY PERCENTAGE BY SOURCE

(Percentages <. $1 \%$ are not displayed)

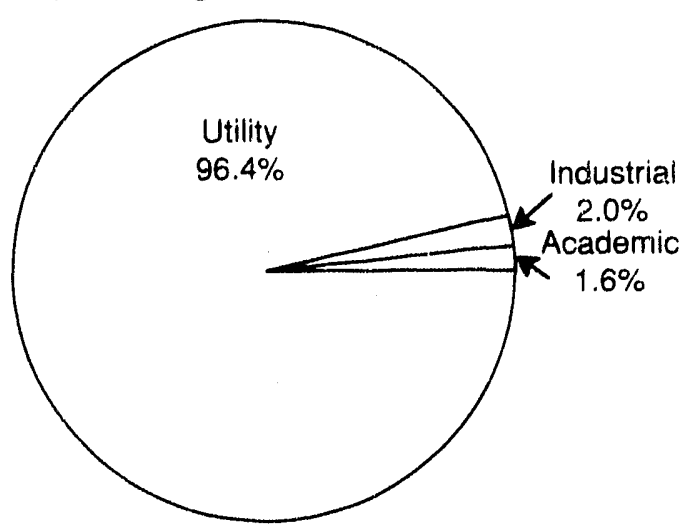




\section{OREGON \\ Low-Level Radioactive Wastes Received at Commercial Disposal Sites}

\begin{tabular}{c} 
Disposal \\
Site \\
\hline
\end{tabular}

Beatty

Richland

Total

\begin{tabular}{c}
$\begin{array}{c}\text { Volume } \\
\left(\mathrm{ft}^{3}\right)\end{array}$ \\
\hline
\end{tabular}

47.18

$75,860.30$

$75,907.48$
Activity

(curies)

2.49

463.26

465.75
VOLUME PERCENTAGE BY DISPOSAL SITE

(Percentages <.1\% are not displayed)

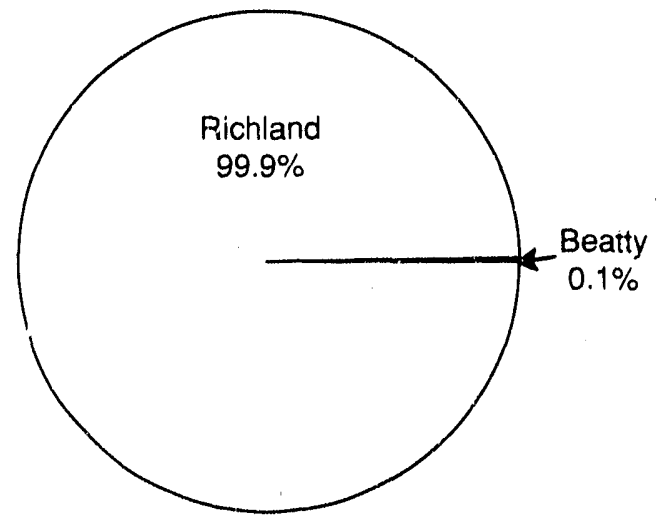

Direct Shipment

Waste Class

$$
\begin{aligned}
& \text { A } \\
& \text { B } \\
& \text { C }
\end{aligned}
$$

Total

Non-Direct Shipments

Grand Total

Total Direct Shipment

Activity (curies)

Total Non-Direct Shipment

Activity (curies)

Grand Total (curies)
ACTIVITY PERCENTAGE BY DISPOSAL SITE (Percentages <. $1 \%$ are not displayed)

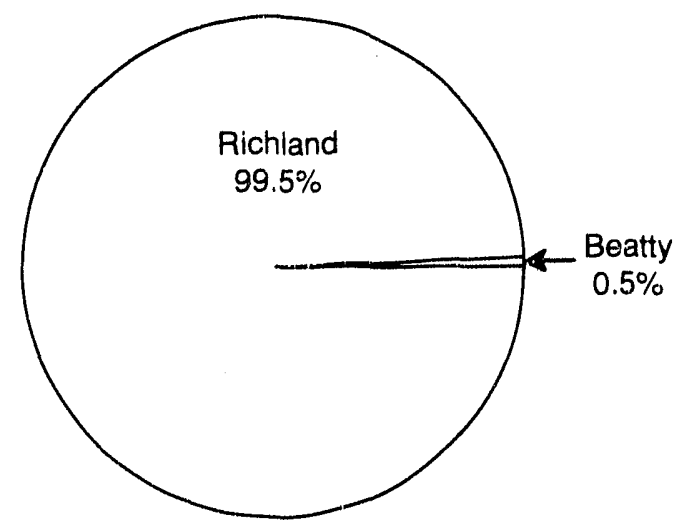

Volume Percentage by Waste Class for Direct Shipments and Total Non-Direct Volume Percentage (Percentages <. $1 \%$ are not displayed)

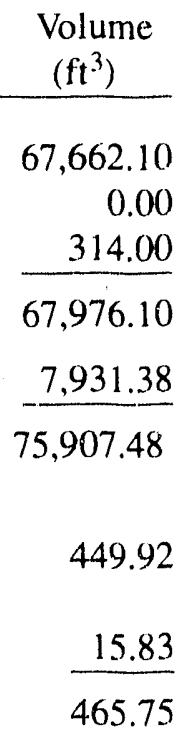

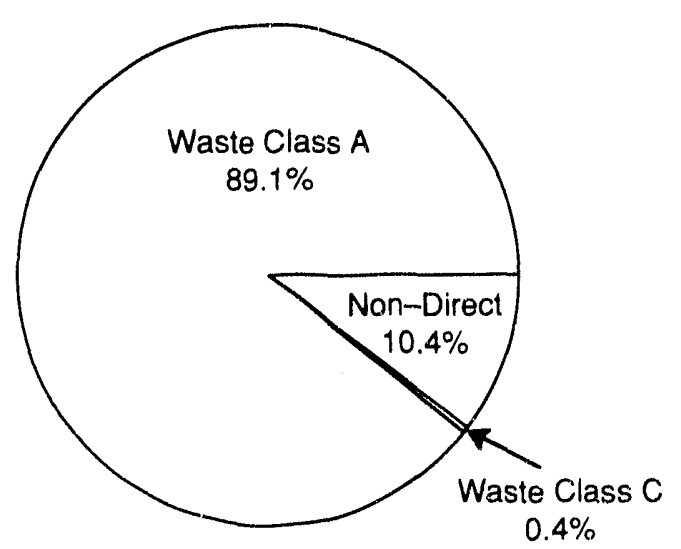




\section{UTAH \\ Low-Level Radioactive Wastes Received at Commercial Disposal Sites}

\begin{tabular}{lrr}
$\begin{array}{l}\text { Generator } \\
\text { Category }\end{array}$ & $\begin{array}{c}\text { Volume } \\
\left(\mathrm{ft}^{3}\right)\end{array}$ & $\begin{array}{r}\text { Activity } \\
\text { (curies) }\end{array}$ \\
\cline { 2 - 3 } $\begin{array}{l}\text { Government } \\
\text { Industrial }\end{array}$ & $\begin{array}{r}7.30 \\
6.292 .01\end{array}$ & $\begin{array}{r}7.40 \\
\text { Total }\end{array}$ \\
\cline { 2 - 3 } & $6,299.31$ & 7.47 \\
\hline
\end{tabular}

VOLUME PERCENTAGE BY SOURCE (Percentages <. $1 \%$ are not displayed)

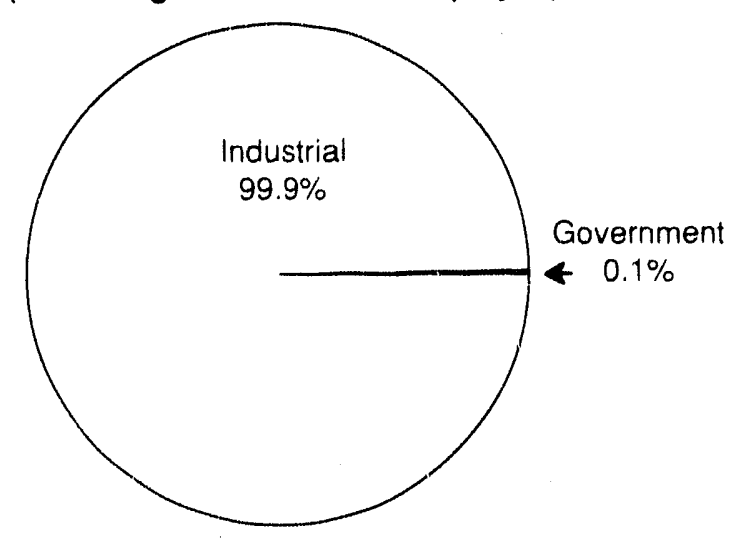

ACTIVITY PERCENTAGE BY SOURCE (Percentages <. $1 \%$ are not displayed)

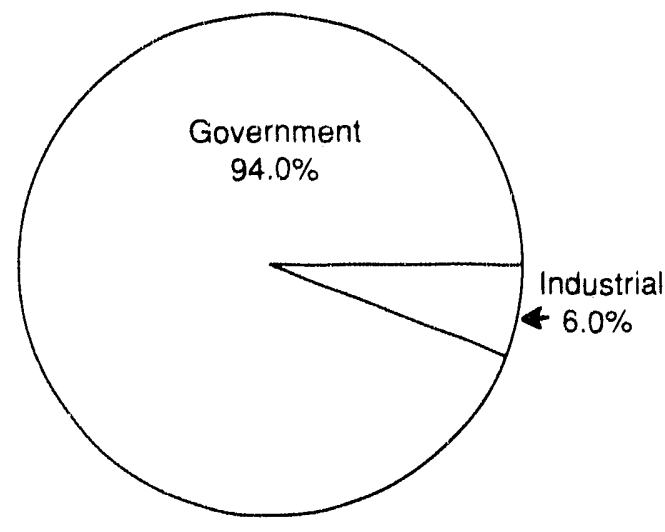




\section{UTAH \\ Low-Level Radioactive Wastes Received at Commercial Disposal Sites}

\begin{tabular}{c} 
Disposal \\
Site \\
\hline
\end{tabular}

Barnwell

Beatty

Richland

Total

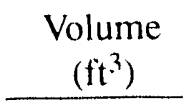

3.00

14.29

$6,282.02$

$6,299.31$
Activity

(curies)

0.22

0.12

7.53

7.88

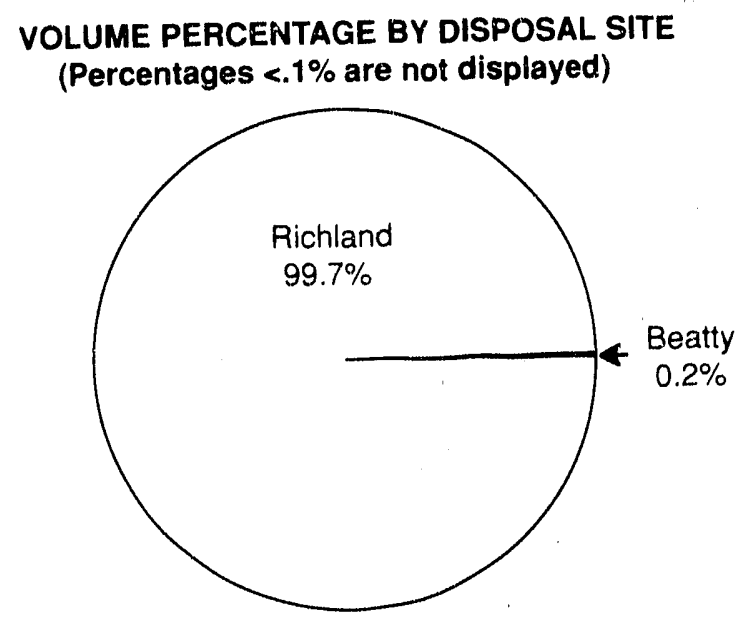

ACTIVITY PERCENTAGE BY DISPOSAL SITE

(Percentages $<.1 \%$ are not displayed)

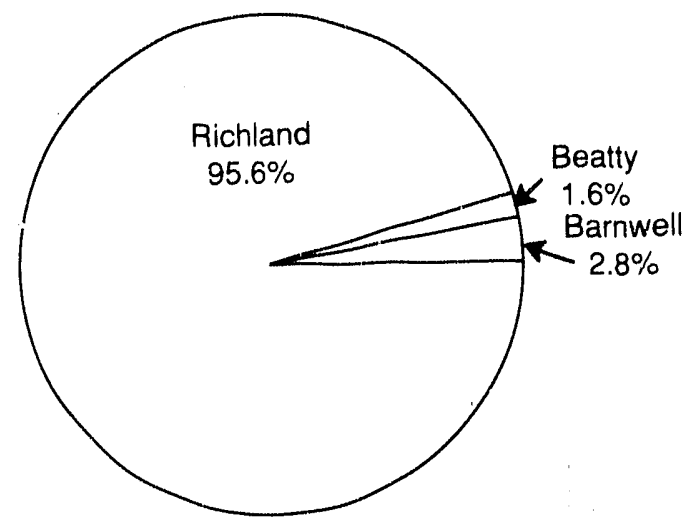

Volume Percentage by Waste Class for Direct Shipments and Total Non-Direct Volume Percentage (Percentages <. $1 \%$ are not displayed)

Direct Shipment

Waste Class

A
B
C

Total

Non-Direct Shipments

Grand Total

Total Direct Shipment

Activity (curies)

Total Non-Direct Shipment

Activity (curies)

Grand Total (curies)
Volume

$\left(\mathrm{ft}^{3}\right)$

$6,268.50$

0.00

0.00

$6,268.50$

30.81

$6,299.31$

0.34

7.53

7.88

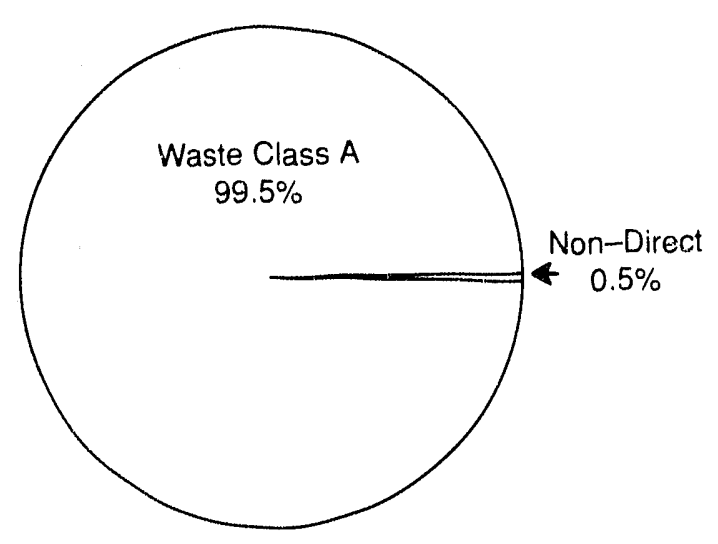




\section{WASHINGTON \\ Low-Level Radioactive Wastes Received at Commercial Disposal Sites}

\begin{tabular}{l} 
Generator \\
Category \\
\hline
\end{tabular}

Academic Government Industrial Medical Utility

Total

$$
\begin{gathered}
\begin{array}{c}
\text { Volume } \\
\left(\mathrm{ft}^{3}\right)
\end{array} \\
\hline 2,029.13 \\
6,830.50 \\
3,573.10 \\
1,136.51 \\
12,839.30
\end{gathered}
$$

$26,408.54$
Activity

(curies)

7.78

11.40

0.32

$1,084.10$

$1,105.04$
VOLUME PERCENTAGE BY SOURCE (Percentages <. $1 \%$ are not displayed)

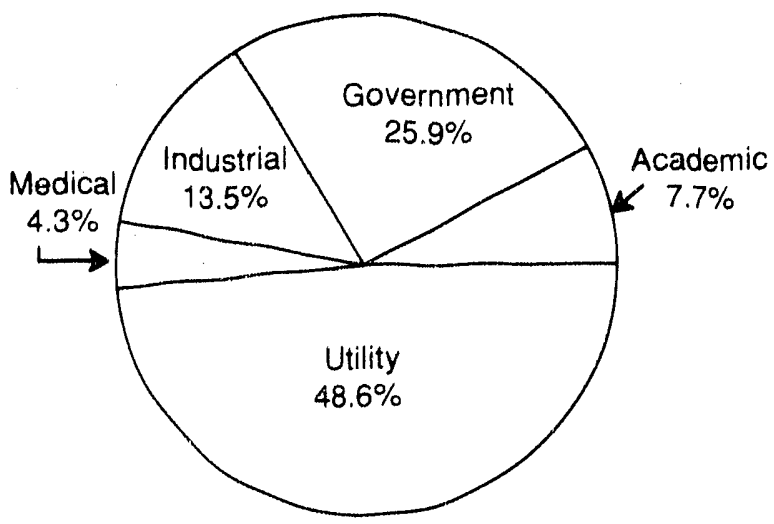

ACTIVITY PERCENTAGE BY SOURCE (Percentages $<.1 \%$ are not displayed)

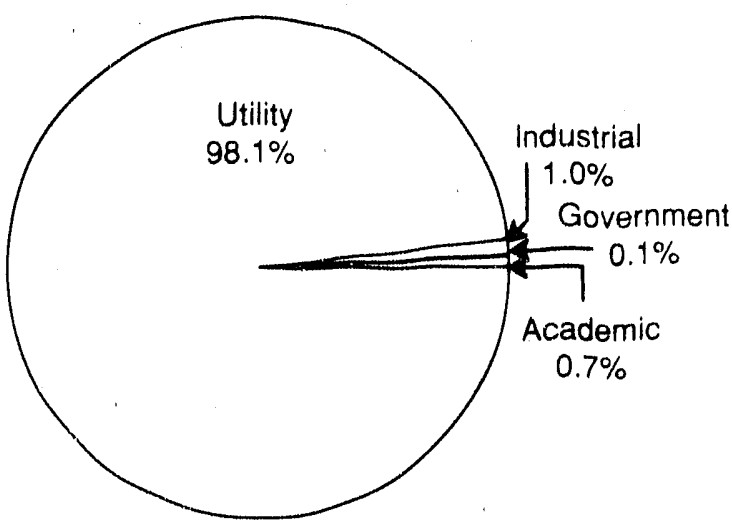




\section{WASHINGTON \\ Low-Level Radioactive Wastes Received at Commercial Disposal Sites}

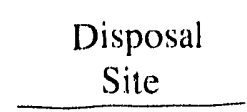

Beatty

Richland

Total

\author{
Volume \\ $\left(\mathrm{ft}^{3}\right)$ \\ 46.00 \\ $26,362.54$ \\ $26,408.54$
}

Activity

(curies)

3.11

$1,101.93$

$1,105.04$ VOLUME PERCENTAGE BY DISPOSAL SITE
(Percentages $<.1 \%$ are not wisplayed)

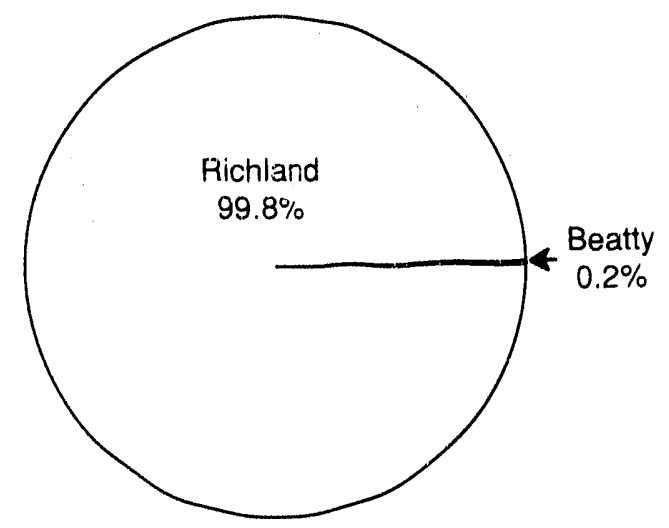

ACTIVITY PERCENTAGE BY DISPOSAL SITE

(Percentages $<.1 \%$ are not displayed)
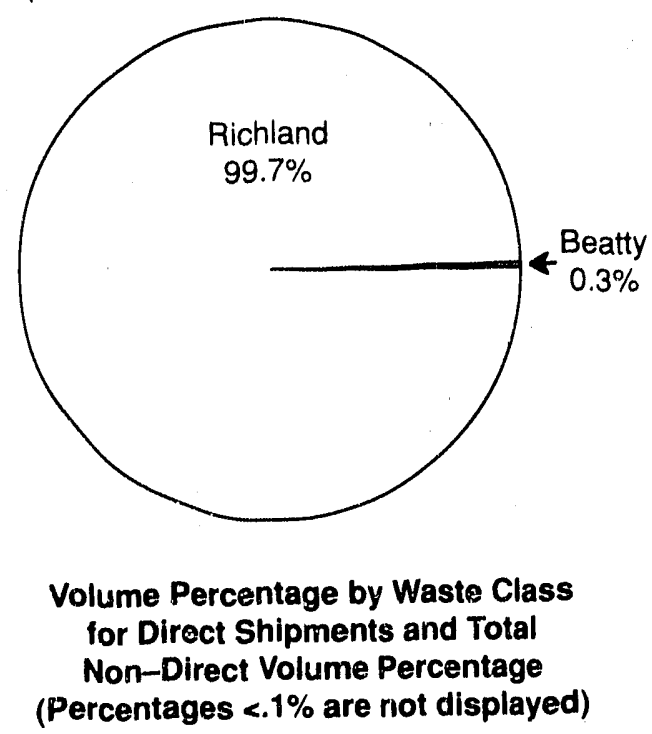

Volume Percentage by Waste Class for Direct Shipments and Total (Percentages <.1\% are not displayed)
Direct Shipment

Waste Class

A

B

C

Total

Non-Direct Shipments

Grand Total

Total Direct Shipment

Activity (curies)

Total Non-Direct Shipment

Activity (curies)

Grand Total (curies)
Volume

$$
\begin{array}{r}
\left(\mathrm{ft}^{3}\right) \\
\hline 22,388.43 \\
131.00 \\
0.00 \\
\hline 22,519.43 \\
\frac{3,889.11}{26,408.54} \\
1,093.35 \\
\frac{11.69}{1,105.04}
\end{array}
$$

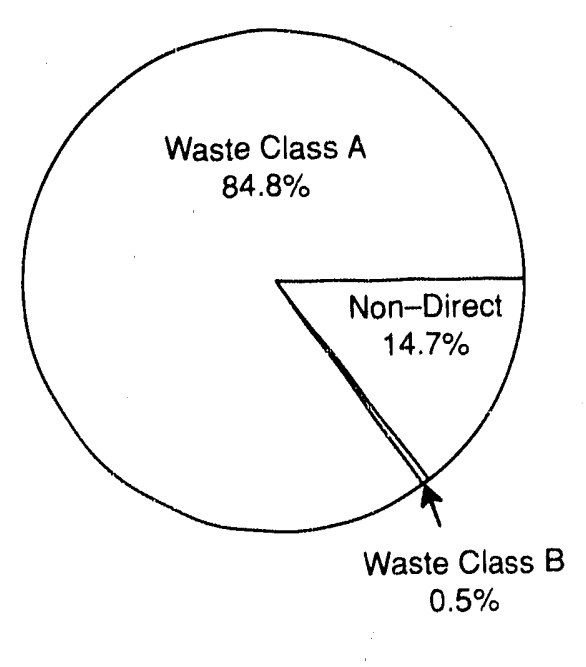




\section{ROCKY MOUNTAIN COMPACT \\ Low-Level Radioactive Wastes Received at Commercial Disposal Sites}

\begin{tabular}{lrr}
\multicolumn{1}{c}{ States } & \multicolumn{1}{c}{$\begin{array}{c}\text { Volume } \\
\left(\mathrm{ft}^{3}{ }^{3}\right.\end{array}$} & \multicolumn{1}{c}{$\begin{array}{c}\text { Activity } \\
\text { (curies) }\end{array}$} \\
\cline { 2 - 3 } Colorado & $8,754.29$ & $1,102.84$ \\
Nevada & 307.65 & 789.32 \\
New Mexico & $1,369.09$ & 94.54 \\
Wyoming & 0.50 & 0.19 \\
Total & $10,431.53$ & $1,986.90$
\end{tabular}

VOLUME PERCENTAGE BY STATE

(Percentages $<.1 \%$ are not displayed)

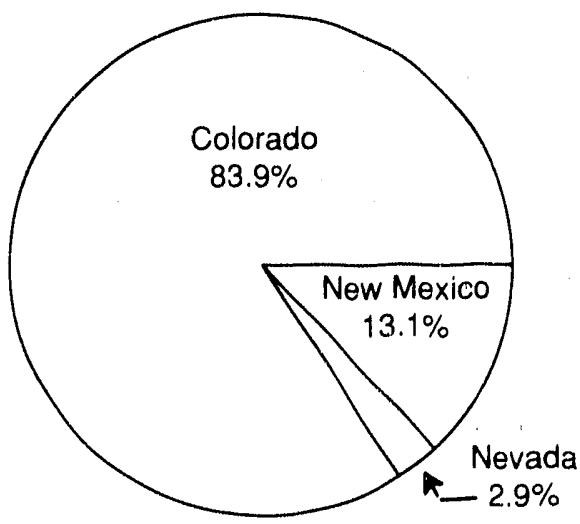

VOLUME PERCENTAGE BY CATEGORY

(Percentages <. $1 \%$ are not displayed)

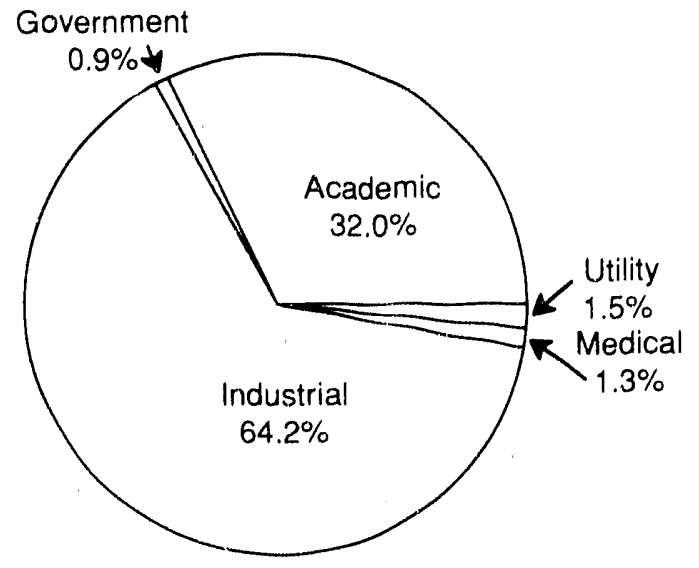


COLORADO

\section{Low-Level Radioactive Wastes Received at Commercial Disposal Sites}

\author{
Generator \\ Category
}

Academic

Government

Industrial

Medical

Utility

Total

$$
\begin{array}{r}
\begin{array}{c}
\text { Volume } \\
\left(\mathrm{ft}^{3}\right)
\end{array} \\
\hline 2,417.03 \\
31.88 \\
6,069.22 \\
74.76 \\
161.40 \\
\hline
\end{array}
$$

$8,754.29$
Activity (curies)

14.74

0.20

$1,079.01$

$1,102.84$
VOLUME PERCENTAGE BY SOURCE (Percentages <. $1 \%$ are not displayed) Government

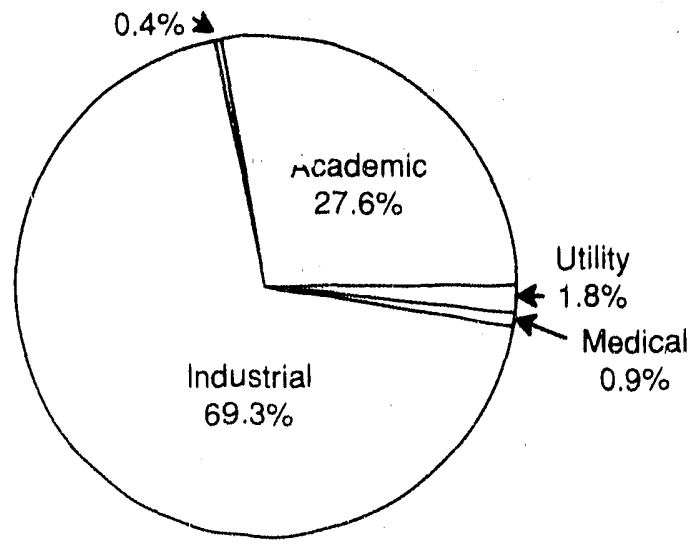

ACTIVITY PERCENTAGE BY SOURCE

(Percentages <.1\% are not displaye )

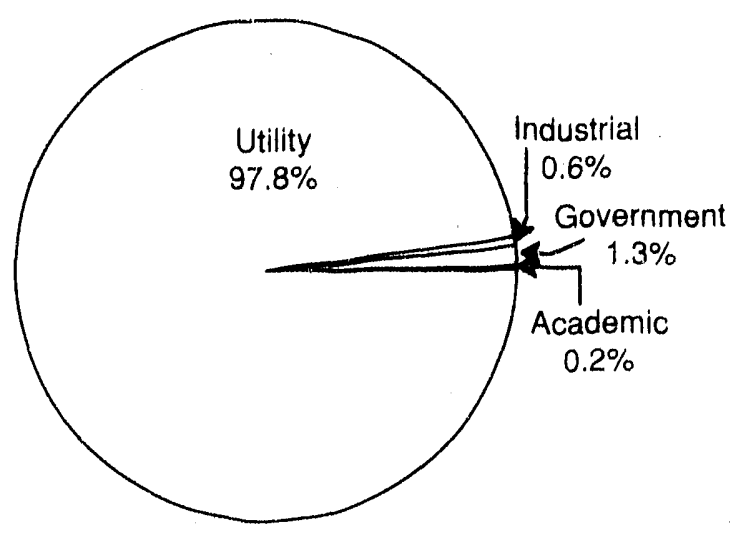




\section{COLORADO \\ Low-Level Radioactive Wastes Received at Commercial Disposal Sites}

\begin{tabular}{l}
$\begin{array}{l}\text { Disposal } \\
\text { Site }\end{array}$ \\
\hline Beatty \\
Richland \\
Total
\end{tabular}

Total

\begin{tabular}{c}
$\begin{array}{c}\text { Volume } \\
\left(\mathrm{ft}^{3}\right)\end{array}$ \\
\hline $\begin{array}{r}3,638.79 \\
5,115.50 \\
\hline 8,754.29\end{array}$
\end{tabular}

Activity (curies)

$1,102.73$

0.11

$1,102.84$
VOLUME PERCENTAGE BY DISPOSAL SITE (Percentages <.1\% are not displayed)

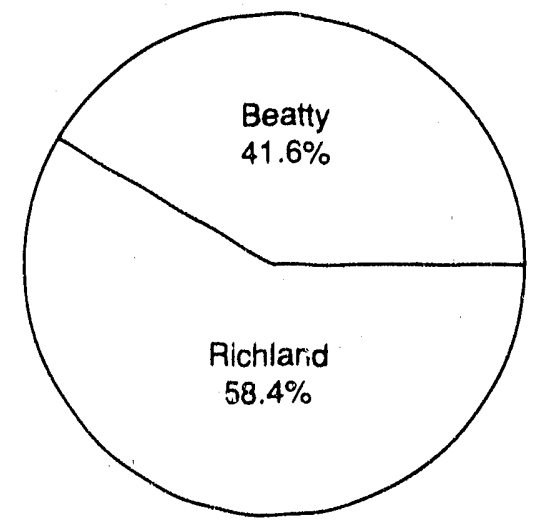

\section{ACTIVITY PERCENTIAGE BY DISPOSAL SITE}

(Percentages <.1\% are not displayed)

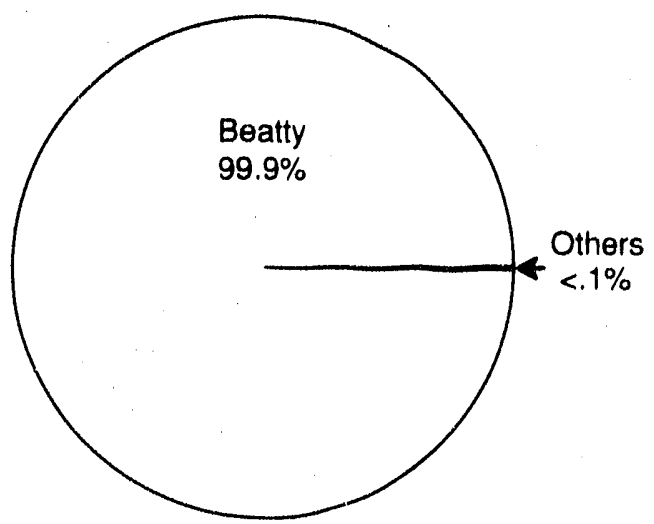

Volume Percentage by Waste Class for Direct Shipments and Total Non-Direct Volume Percentage (Percentages <.1\% are not displayed)
Direct Shipment Waste Class
A
B
C

Total

Non-Direct Shipments

Grand Total

Total Direct Shipment

Activity (curies)

Total Non-Direct Shipment

Activity (curies)

Grand Total (curies)
Volume $\left(\mathrm{ft}^{3}\right)$

$\begin{array}{r}\left(\mathrm{ft}^{3}\right) \\ 0.00 \\ 0.00 \\ \frac{60.40}{60.40} \\ \frac{8,693.89}{8,754.29} \\ 1,079.00 \\ 23.84 \\ \hline 1,102.84\end{array}$

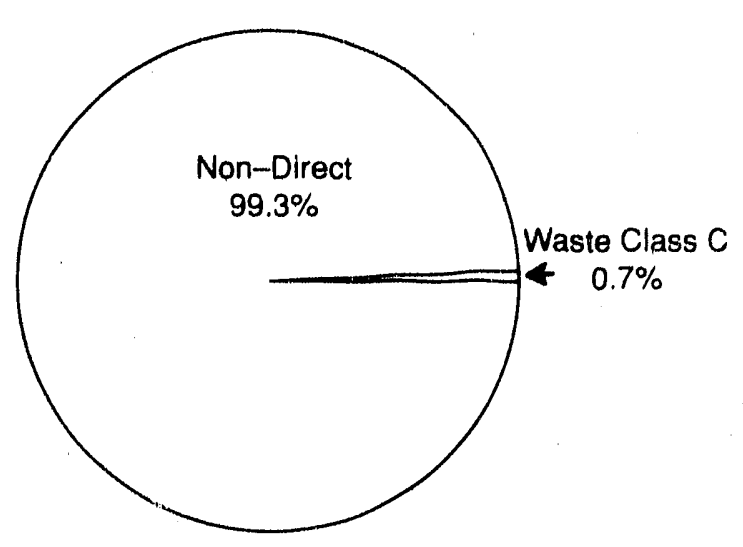




\section{NEVADA \\ Low-Level Radioactive Wastes Received at Commercial Disposal Sites}

\begin{tabular}{l} 
Generator \\
Category \\
\hline
\end{tabular}

Academic

Government

Industrial

Medical

Total

$\begin{array}{r}\begin{array}{c}\text { Volume } \\ \left(\mathrm{ft}^{3}\right)\end{array} \\ \hline 240.35 \\ 39.80 \\ 22.50 \\ 5.00 \\ \hline\end{array}$

307.65
Activity

(curies)

$$
0.09
$$

786.69

2.54

$<1$

789.32

VOLUME PERCENTAGE BY SOURCE

ACTIVITY PERCENTAGE BY SOURCE

(Percentages <.1\% are not displayed)

(Percentages $<.1 \%$ are not displayed)
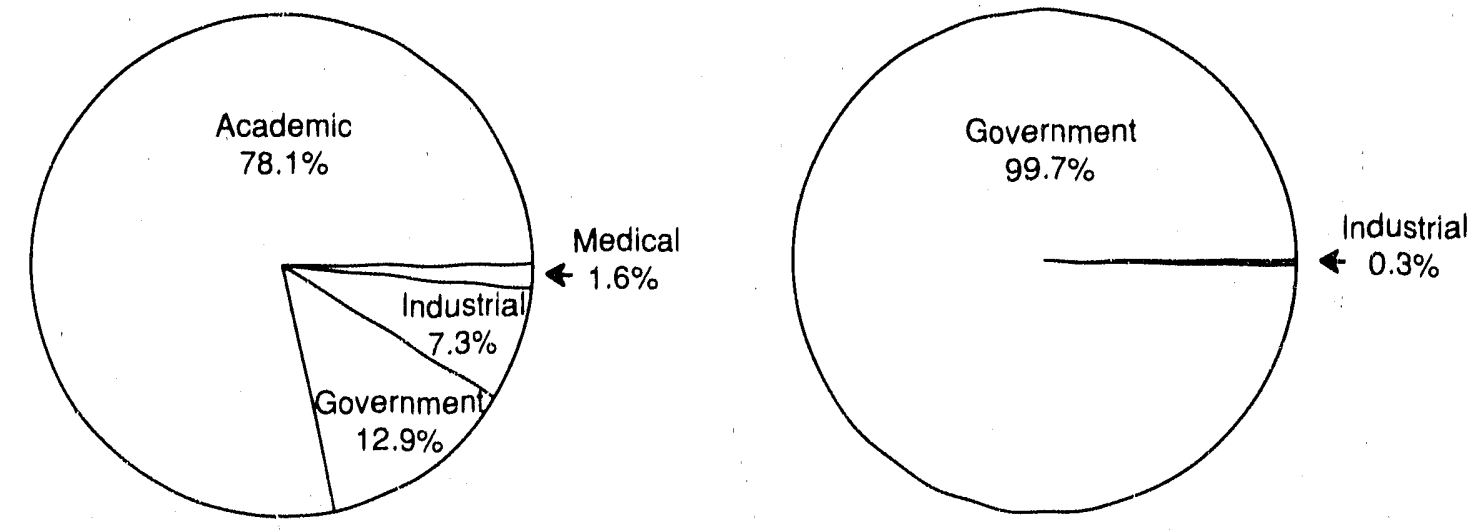


\section{NEVADA \\ Low-Level Radioactive Wastes Received at Commercial Disposal Sites}

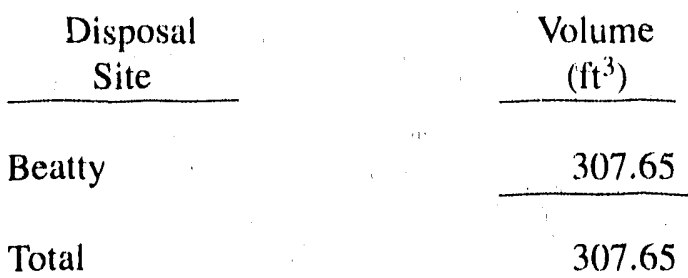

Activity

(curies)

789.32

789.32
VOLUME PERCENTAGE BY DISPOSAL SITE

(Percentages <. $1 \%$ are not displayed)

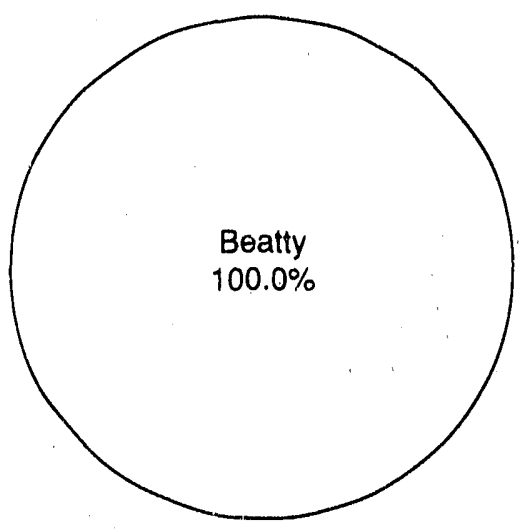

ACTIVITY PERCENTAGE BY DISPOSAL SITE

(Percentages <. $1 \%$ are not clisplayed)

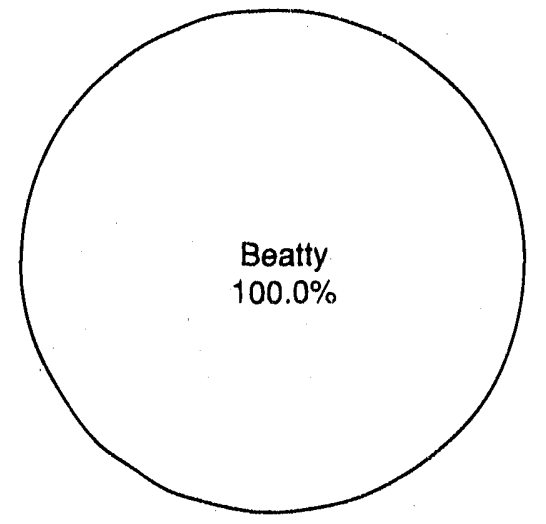

Volume Percentage by Waste Class for Direct Shipments and Total

Non-Direct Volume Percentage

(Percentages <. $1 \%$ are not displayed)

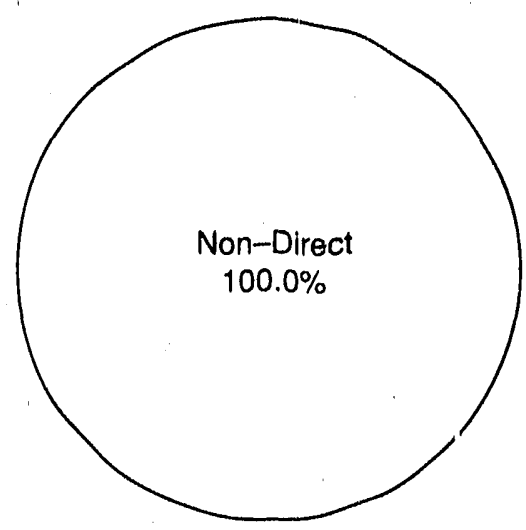




\section{NEW MEXICO \\ Low-Level Radioactive Wastes Received at Commercial Disposal Sites}

\begin{tabular}{l}
$\begin{array}{l}\text { Generator } \\
\text { Category }\end{array}$ \\
\hline Academic \\
Government \\
Industrial \\
" tical \\
'Iutal
\end{tabular}

VOLUME PERCENTAGE BY SOURCE

(Percentages <. $1 \%$ are not displayed)

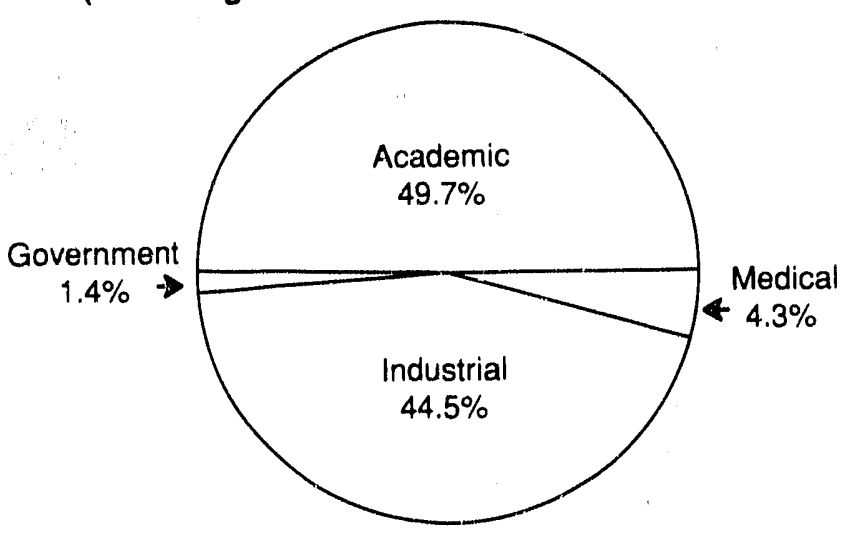

Activity

(curies)

$\begin{array}{r}\multicolumn{1}{c}{\begin{array}{r}\text { Volume } \\ \left(\mathrm{ft}^{3}\right)\end{array}} \\ \hline 681.11 \\ 19.10 \\ 609.58 \\ 59.30 \\ \hline 1,369.09\end{array}$

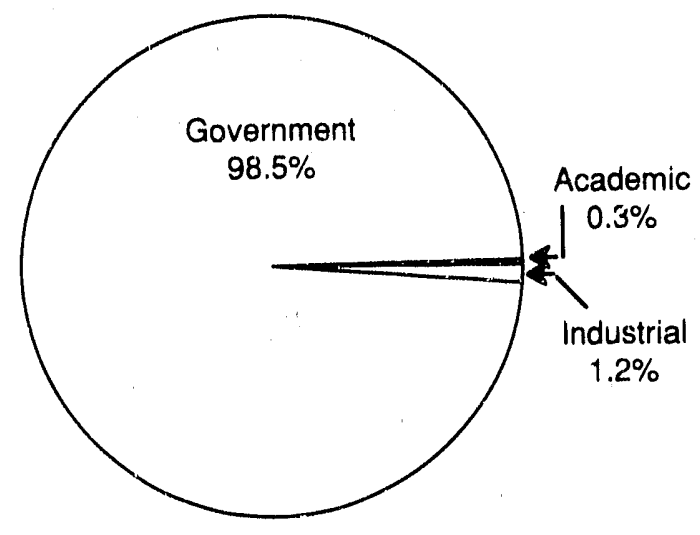




\section{NEW MEXICO \\ Low-Level Radioactive Wastes Received at Commercial Disposal Sites}

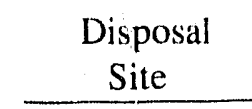

Beatty

Richland

Total

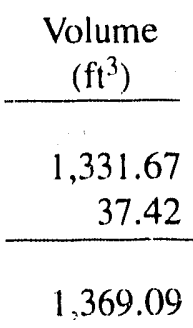

Activity

(curies)

94.52

0.03

94.54
VOLUME PERCENTAGE BY DISPOSAL SITE (Percentages <. $1 \%$ are not displayed)

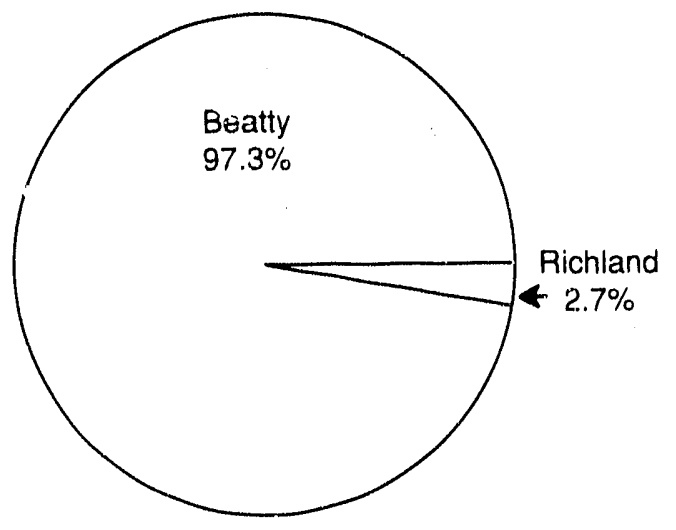

Direct Shipment

Waste Class

A

B

C

Total

Non-Direct Shipments

Grand Total

Total Direct Shipment

Activity (curies)

Total Non-Direct Shipment

Activity (curies)

Grand Total (curies;
$1,369.09$

94.23

Volume $\left(\mathrm{ft}^{3}\right)$

677.10

0.00

0.00

677.10

691.99

$1,369.09$

0.31

94.54
ACTIVITY PERCENTAGE BV DISPOSAL SITE

(Percentages <. $1 \%$ are not displayed)

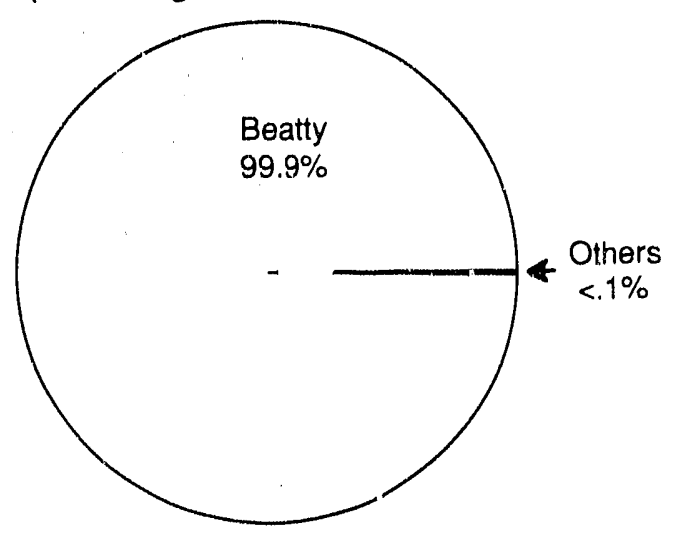

Volume Percentage by Waste Class for Direct Shipments and Total Non-Direct Volume Percentage (Percentages <. $1 \%$ are not displayed)

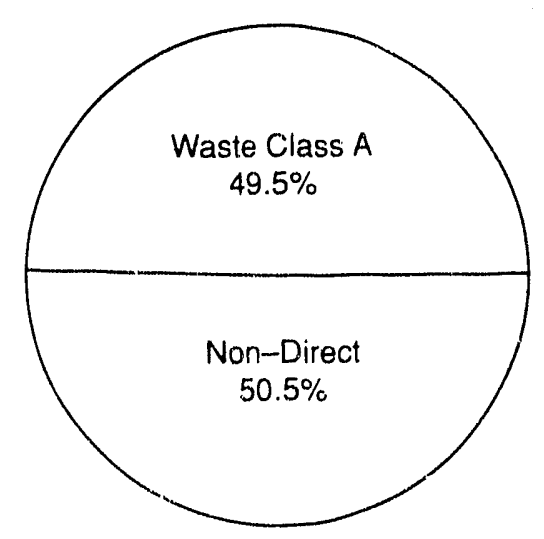




\section{WYOMING \\ Low-Level Radioactive Wastes Received at Commercial Disposal Sites}

\begin{tabular}{lcc}
$\begin{array}{l}\text { Generator } \\
\text { Cu egory }\end{array}$ & $\begin{array}{c}\text { Volume } \\
\left(\mathrm{ft}^{3}{ }^{3}\right)\end{array}$ & $\begin{array}{c}\text { Activity } \\
\text { (curies) }\end{array}$ \\
\cline { 1 - 2 } Government & 0.50 & 0.19 \\
Total & 0.50 & 0.19
\end{tabular}

VOLUME PERCENTAGE BY SOURCE

ACTIVITY PERCENTAGE BY SOURCE

(Percentages $<.1 \%$ are not displayed)

(Percentages <. $1 \%$ are not displayed)
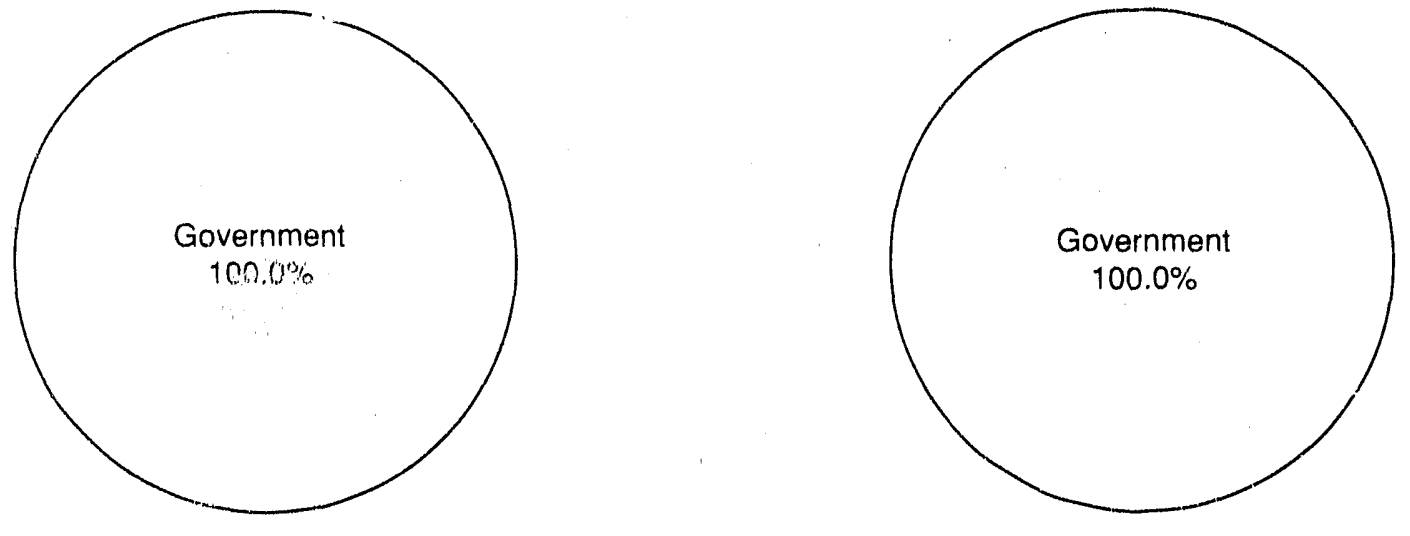


\section{WYOMING \\ Low-Level Radioactive Wastes Received at Commercial Disposal Sites}

Disposal
Site

Beatty

Total

\author{
Volume \\ $\left(\mathrm{ft}^{3}\right)$
}

0.50

0.50
Activity

(curies)

0.19

0.19
VOLUME PERCENTAGE BY DISPOSAL SITE

(Percentages $<.1 \%$ are not displayed)

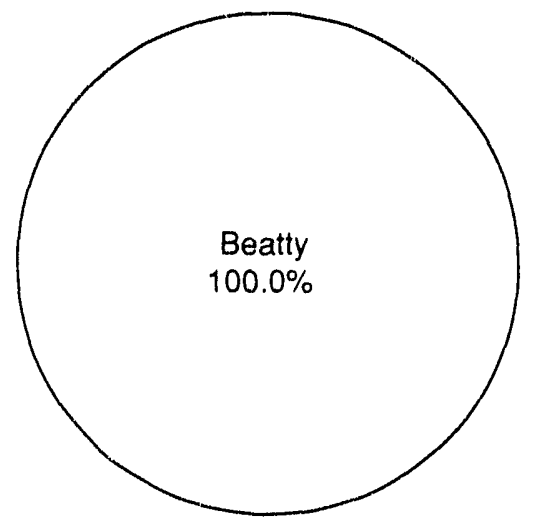

ACTIVITY PERCENTAGE BY DISPOSAL SITE

(Percentages <. $1 \%$ are not displayed)

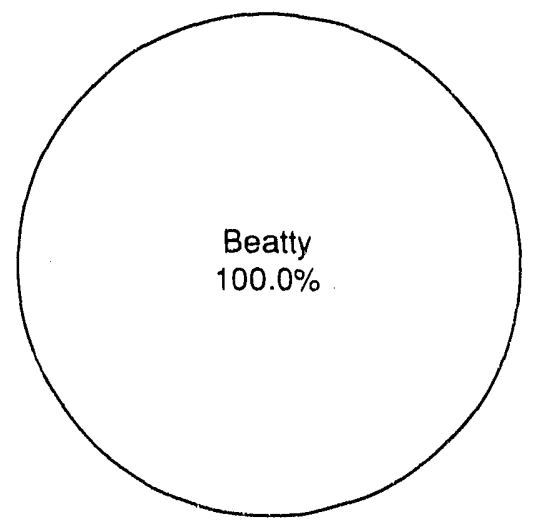

Volume Percentage by Waste Class

for Direct Shipments and Total

Non-Direct Volume Percentage

(Percentages $<.1 \%$ are not displayed)

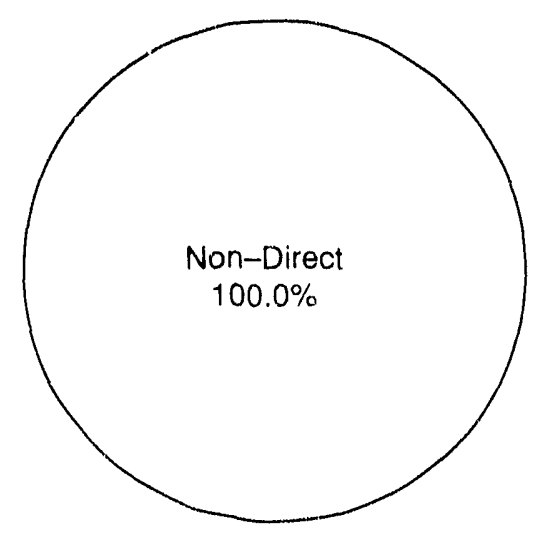

A-77/78 


\section{SOUTHEAST COMPACT Low-Level Radioactive Wastes Received at Commercial Disposal Sites}

States

Alabama

Florida

Georgia

Mississippi

North Carolina

South Carolina

Tennessee

Virginia

Total

\begin{tabular}{c}
$\begin{array}{c}\text { Volume } \\
\left(\mathrm{ft}^{3}\right)\end{array}$ \\
\hline
\end{tabular}

$49,249.06$

$35,282.54$

$44,697.36$

$10,995.47$

$58,421.49$

$96,695.37$

$118,957.13$

$82,834.30$

$497,132.72$
Activity

(curies)

$1,163.55$

$2,624.41$

$2,887.45$

222.45

$7,390.08$

$2,430.99$

$3,043.58$

$3,053.52$

$22,816.04$

VOLUME PERCENTAGE BY STATE

(Percentages <. $1 \%$ are not displayed)

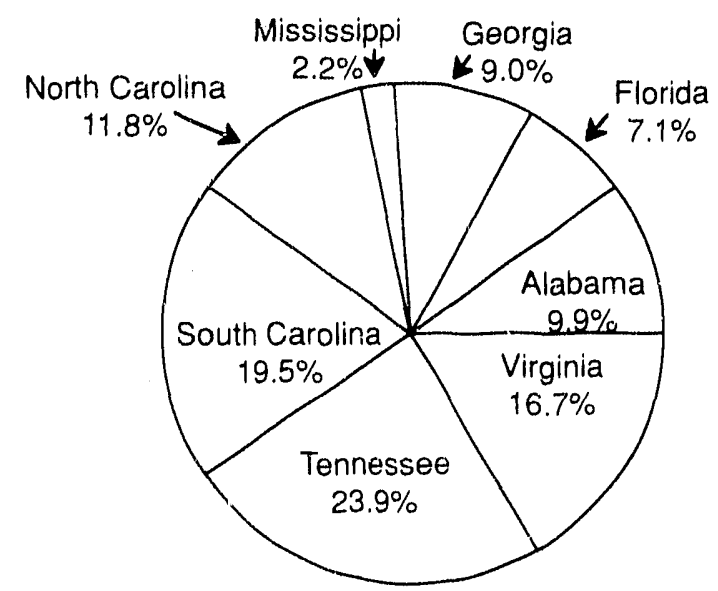

VOLUME PERCENTAGE BY CATEGORY

(Percentages <. $1 \%$ are not displayed)

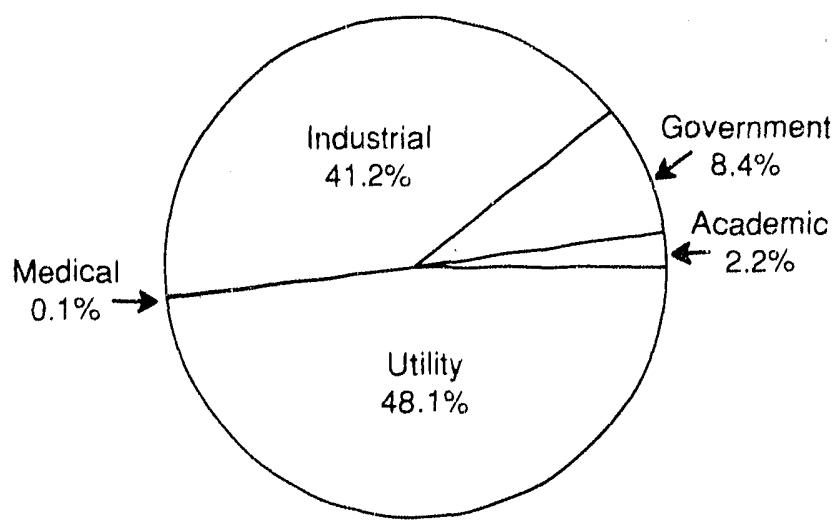

A-79 


\section{ALABAMA \\ Low-Level Radioactive Wastes Received at Commercial Disposal Sites}

\begin{tabular}{lrr}
$\begin{array}{c}\text { Generator } \\
\text { Category }\end{array}$ & \multicolumn{1}{c}{$\begin{array}{c}\text { Volume } \\
\left(\mathrm{ft}^{3}{ }^{3}\right.\end{array}$} & \multicolumn{1}{c}{$\begin{array}{c}\text { Activity } \\
\text { (curies) }\end{array}$} \\
\hline Academic & 213.80 & \multicolumn{1}{c}{$0.70)$} \\
Government & $6,209.11$ & 468.67 \\
Industrial & 5.832 .51 & 0.38 \\
Medical & 27.64 & 0.33 \\
Utility & $36,966.00$ & 693.47 \\
& & \\
Total & $49,249.06$ & $1,163.55$
\end{tabular}

VOLUME PERCENTAGE BY SOURCE (Percentages <. $1 \%$ are not displayed)

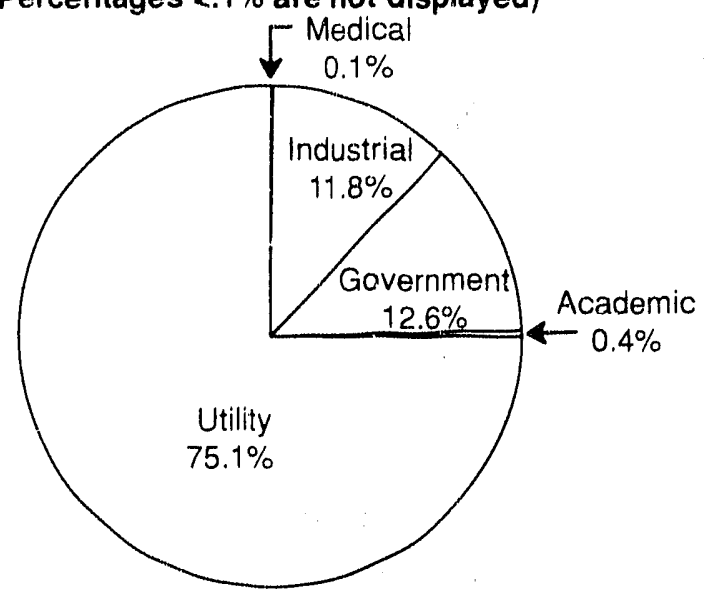

ACTIVITY PERCENTAGE BY SOURCE

(Percentages $<.1 \%$ are not displayed)

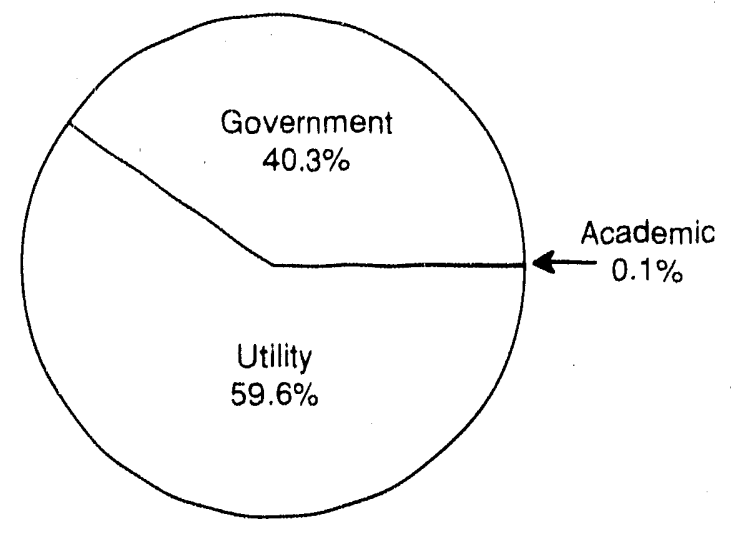




\section{ALABAMA \\ Low-Level Radioactive Wastes Received at Commercial Disposal Sites}

\begin{tabular}{c} 
Disposal \\
Site \\
\hline
\end{tabular}

Barnwell

Beatly

Richland

Total

\author{
Volume \\ $\left(\mathrm{ft}^{3}\right)$
}

$46,895.06$

14.00

$2,340.00$

$49,249.06$
Activity

(curies)

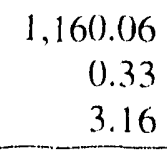

$1,163.55$
VOLUME PERCENTAGE BY DISPOSAL SITE

(Percentages <.1\% are not displayed)

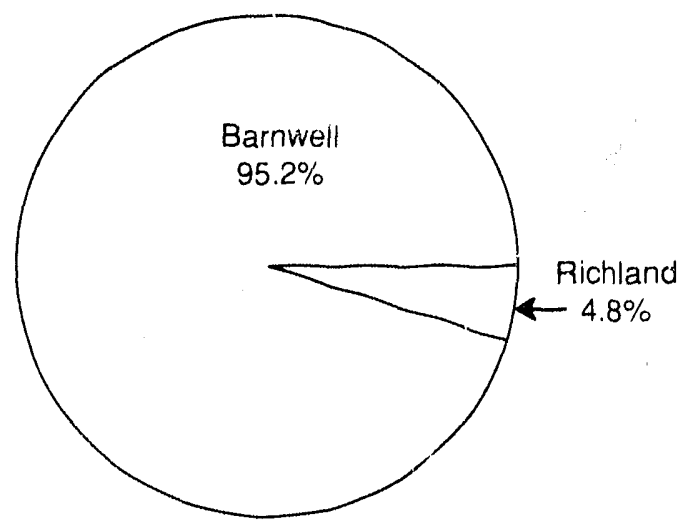

Direct Shipment

Waste Class

A
B
C

Total

Non-Direct Shipments

Grand Total

Total Direct Shipment

Activity (curies)

Tota: Non-Direct Shipment

Activity (curies)

Grand Total (curies)

Volume $\left(\mathrm{ft}^{3}\right)$

$43,803.30$

558.40

0.00

$44,361.70$

$4,887.36$

$49,249.06$

672.98

490.58
ACTIVITY PERCENTAGE BY DISPOSAL SITE

(Percentages $<.1 \%$ are not displayed)

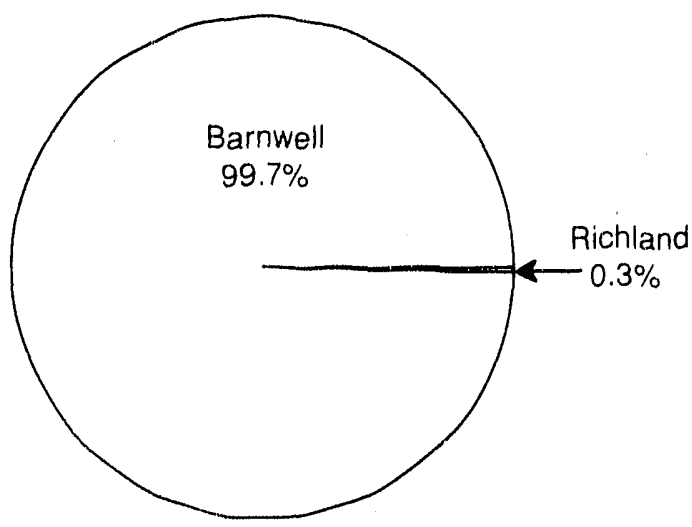

Volume Percentage by Waste Class for Direct Shipments and Total Non-Direct Volume Percentage (Percentages $<.1 \%$ are not displayed)

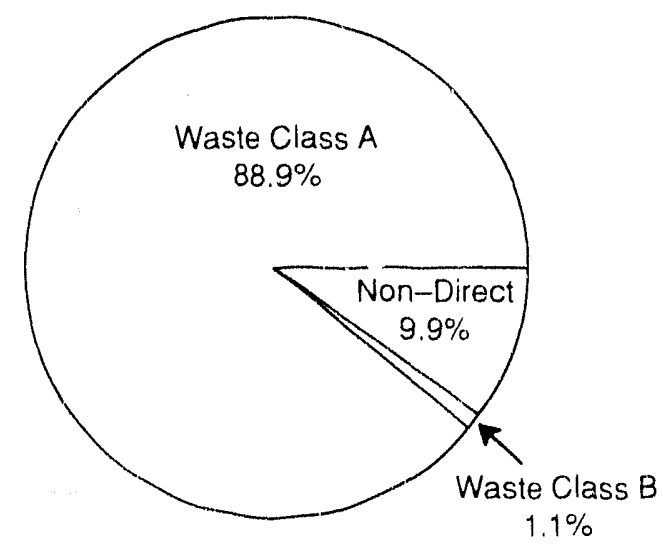




\section{FLORIDA \\ Low-Level Radioactive Wastes Received at Commercial Disposal Sites}

\section{Generator \\ Category}

Academic

Government

Industrial

Medical

Utility

Total

$$
\begin{gathered}
\text { Volume } \\
\left(\mathrm{ft}^{3}\right)
\end{gathered}
$$

913.83

$1,379.60$

$2,112.35$

18.06

$30,858.70$

$35,282.54$
Activity

(curies)

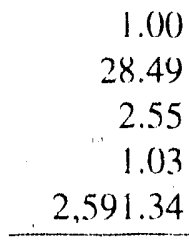

2.624 .41
VOLUME PERCENTAGE BY SOURCE

(Percentages $<.1 \%$ are not displayed)

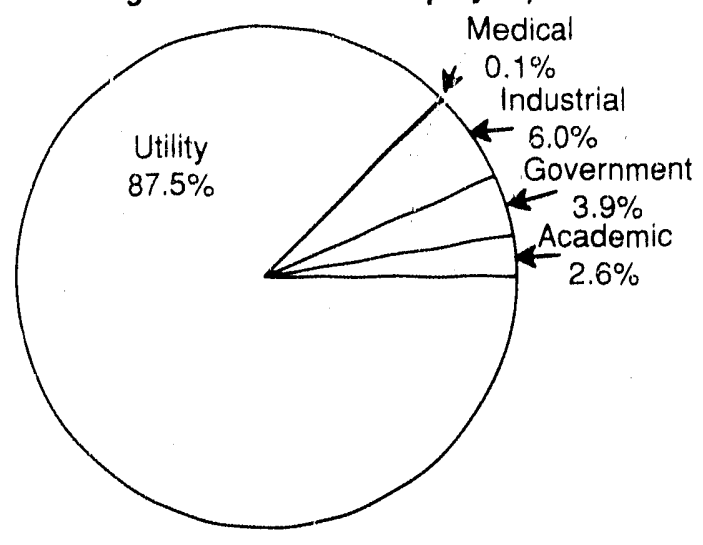

ACTIVITY PERCENTAGE BY SOURCE

(Percentages <.1\% are not displayed)

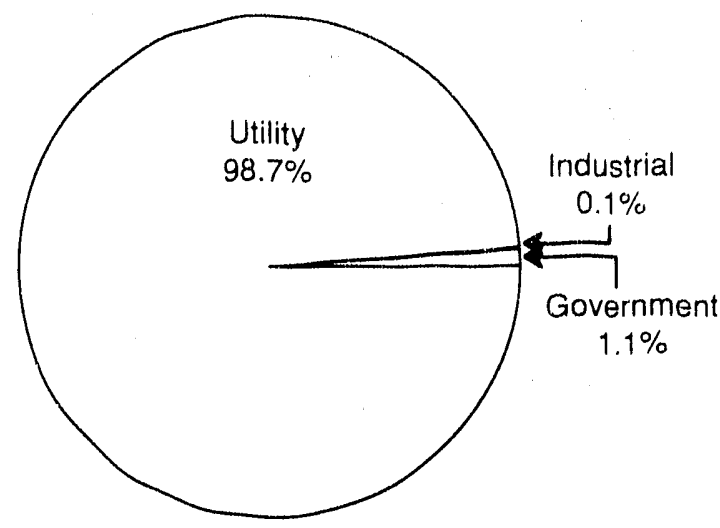




\section{FLORIDA \\ Low-Level Radioactive Wastes Received at Commercial Disposal Sites}

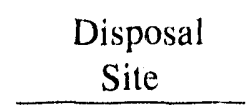

Barnwell

Beatty

Richland

Total

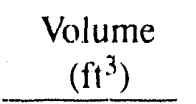

$35,255.02$

12.52

15.00

$35 ; 282.54$
Activity

(curies)

$$
2,621.51
$$

1.03

1.87

$2,624.41$
VOLUME PERCENTAGE BY DISPOSAL SITE

(Percentages <. $1 \%$ are not displayed)

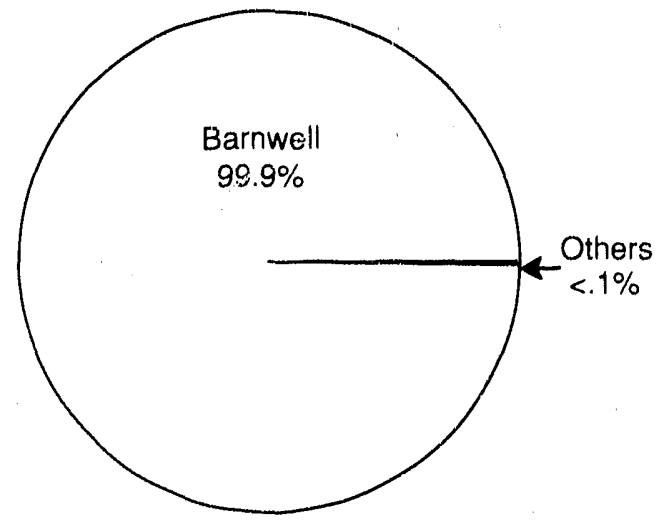

Direct Shipment

Waste Class

A
B
C

Total

Non-Direct Shipments

Grand Total

Total Direct Shipment

Activity (curies)

Total Non-Direct Shipment

Activity (curies)

Grand Total (curies)
Volume

$\left(\mathrm{ft}^{3}\right)$

$10,208.10$

446.40

846.20

$11,500.70$

$23,781.84$

$35,282.54$

$2,584.15$

40.26

$2,624.41$
ACTIVITY PERCENTAGE BY DISPOSAL SITE

(Percentages $<.1 \%$ are not displayed)

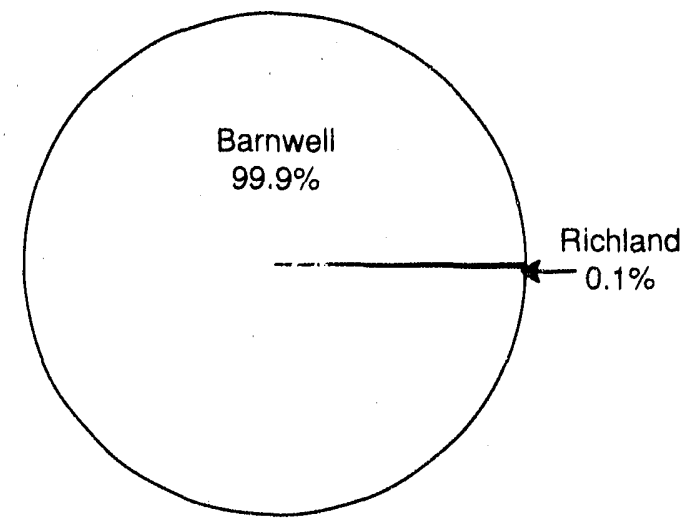

Volume Percentage by Waste Class for Direct Shipments and Total Non-Direct Volume Percentage (Percentages <. $1 \%$ are not displayed)

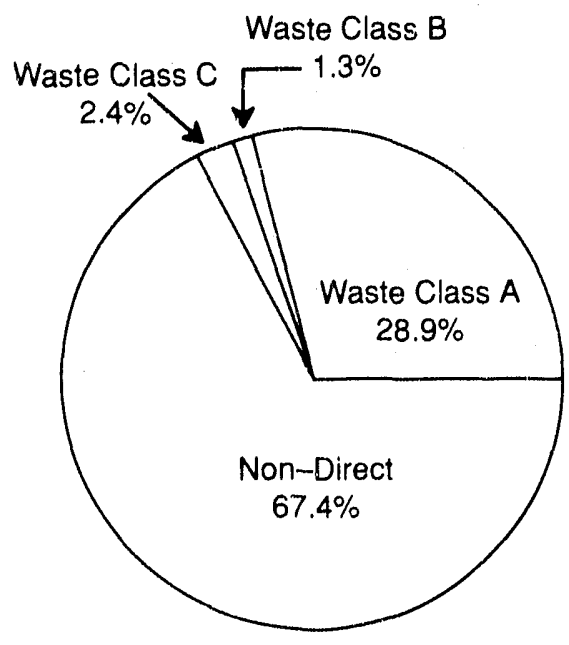




\section{GEORGIA \\ Low-Level Radioactive Wastes Received at Commercial Disposal Sites}

\author{
Generator \\ Category
}

Academic

Government

Industrial

Medical

Utility

Total
Volume

$\left(\mathrm{ft}^{3}\right)$

824.74

456.70

$4,426.80$

0.68

$38,988.44$

$44,697.36$
Activity

(curies)

66.15

890.65

5.10

$<1$

$1,925.55$

$2,887.45$
VOLUME PERCENTAGE BY SOURCE (Percentages <. $1 \%$ are not displayed)

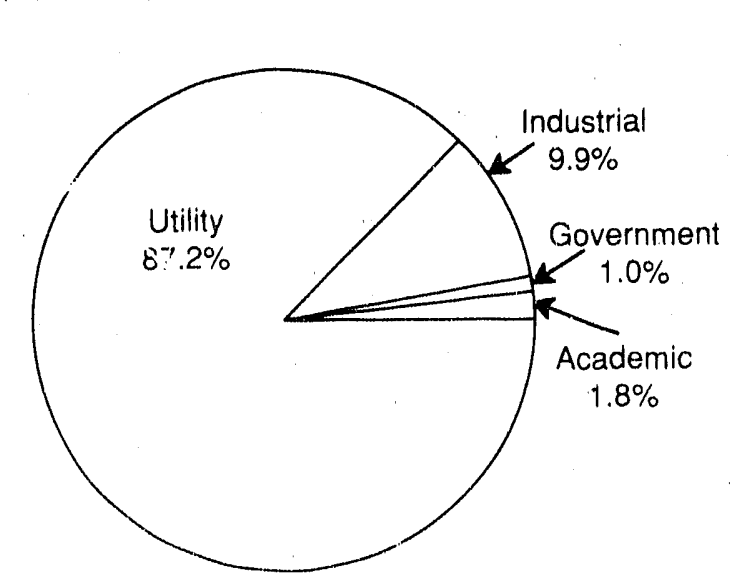

ACTIVITY PERCENTAGE BY SOURCE

(Percentages <.1\% are not displayed)

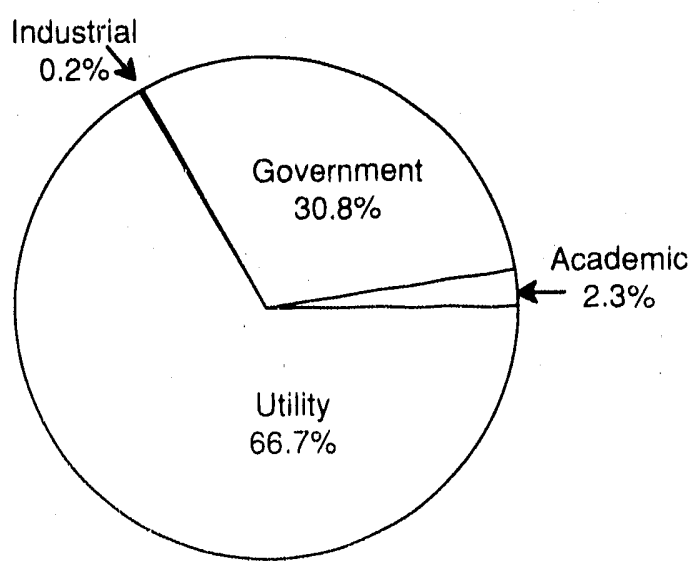




\section{GEORGIA \\ Low-Level Radioactive Wastes Received at Commercial Disposal Sites}

\begin{tabular}{|c|c|c|}
\hline $\begin{array}{c}\text { Disposal } \\
\text { Site } \\
\end{array}$ & $\begin{array}{c}\text { Volume } \\
\left(\mathrm{ft}^{3}\right)\end{array}$ & $\begin{array}{l}\text { Activity } \\
\text { (curies) }\end{array}$ \\
\hline Barnwell & $44,517 . ? 9$ & $2,885.39$ \\
\hline Beatty & 21.89 & 2.03 \\
\hline Richland & 158.18 & 0.03 \\
\hline Total & $44,697.36$ & $2,887.45$ \\
\hline
\end{tabular}

VOLUME PERCENTAGE BY DISPOSAL SITE (Percentages <.1\% are not displayed)

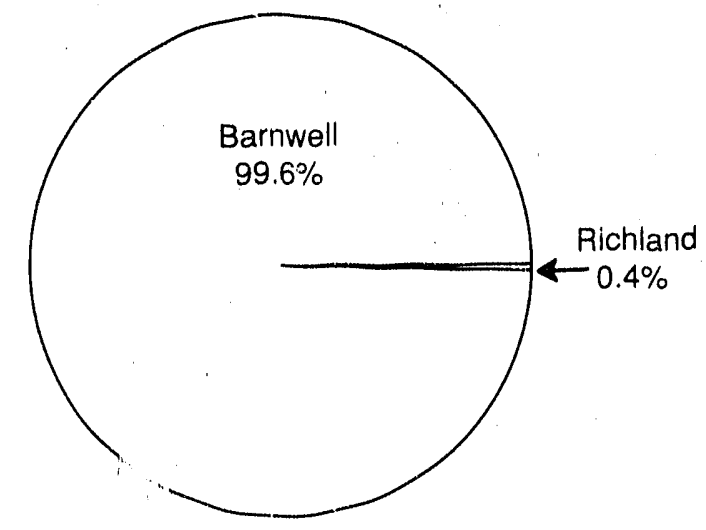

Direct Shipment

Waste Class
A
B
C

Total

Non-Direct Shipments

Grand Total

Total Direct Shipment

Activity (curies)

Total Non-Direct Shipment

Activity (curies)

Grand Total (curies)
Volume

$$
\begin{array}{r}
\frac{\left(\mathrm{ft}^{3}\right)}{34,529.20} \\
1,003.50 \\
\hline 80.70 \\
\hline 35,613.40 \\
9,083.96 \\
\hline 44,697.36 \\
1,928.64 \\
9588.81 \\
2,887.45
\end{array}
$$

ACTIVITY PERCENTAGE BY DISPOSAL SITE

(Percentages <.1\% are not displayed)
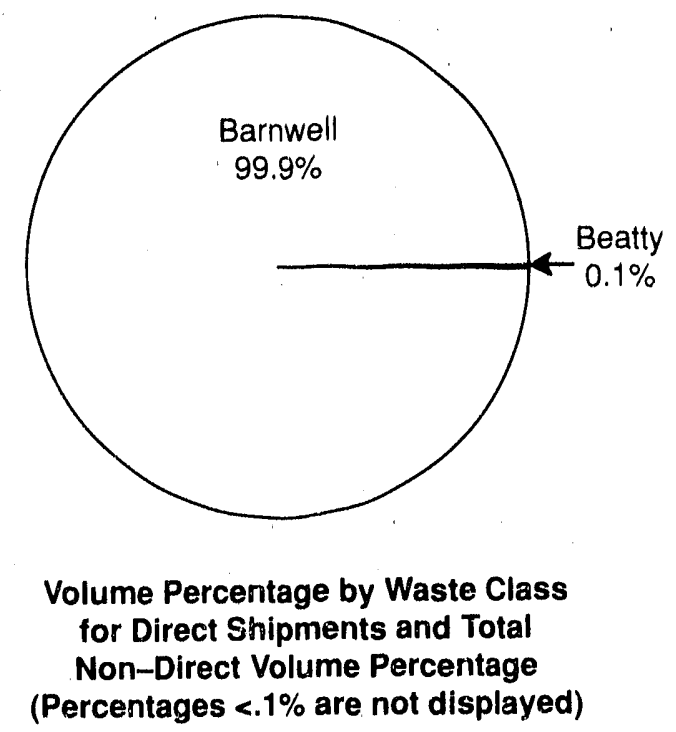

(Percentages $<.1 \%$ are not displayed)

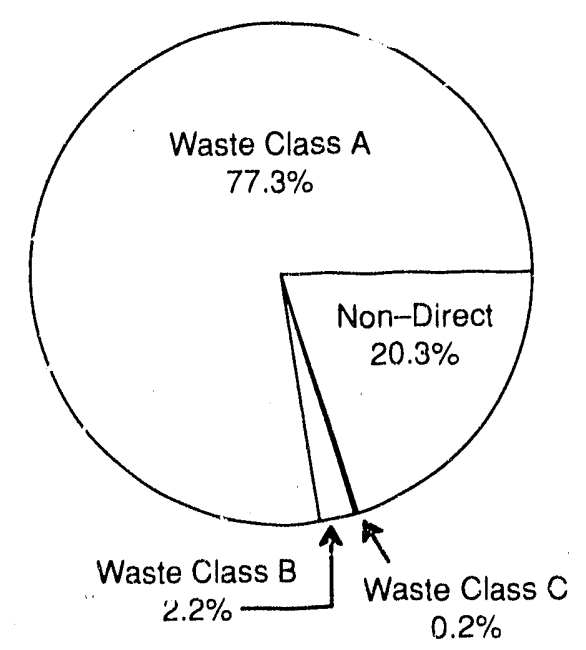




\section{MISSISSIPPI \\ Low-Level Radioactive Wastes Received at Commercial Disposa! Sites}

\section{Generator Category}

Academic Government Industrial Medical Utility

Total

$\begin{array}{r}\begin{array}{c}\text { Volume } \\ \left(\mathrm{ft}^{3}\right)\end{array} \\ \hline 121.36 \\ 48.90 \\ 1,192.70 \\ 5.10 \\ 9,627.41 \\ \hline\end{array}$

$10,995.47$
Activity

(curies)

1.04

15.18

0.94

0.04

205.25

222.45
VOLUME PERCENTAGE BY SOURCE (Percentages <. $1 \%$ are not displayed)

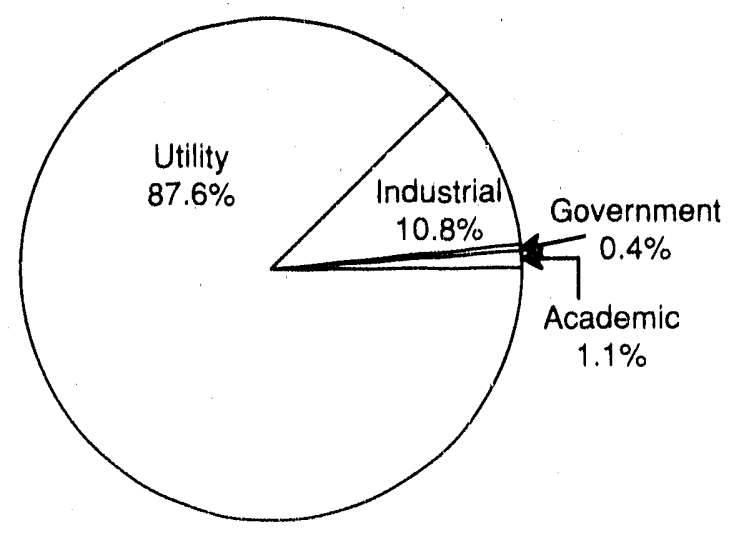

ACTIVITY PERCENTAGE BY SOURCE

(Percentages <.1\% are not displayed)

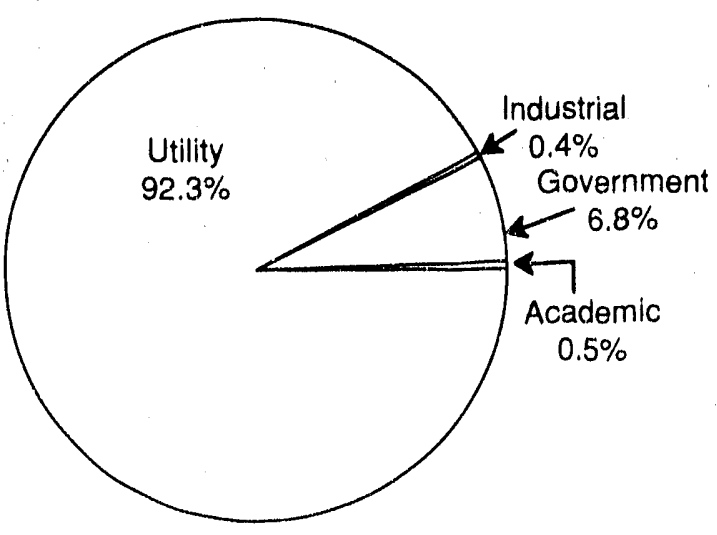




\section{MISSISSIPPI \\ Low-Level Radioactive Wastes Received at Commercial Disposal Sites}

\begin{tabular}{c} 
Disposal \\
Site \\
\hline
\end{tabular}

Barnwell

Beatty

Total

Volume
$\left(\mathrm{ft}^{3}\right)$

$10,542.69$

452.78

$10,995.47$
Activity

(curies)

222.32

0.14

222.45
VOLUME PERCENTAGE BY DISPOSAL SITE (Percentages $<.1 \%$ are not displayed)

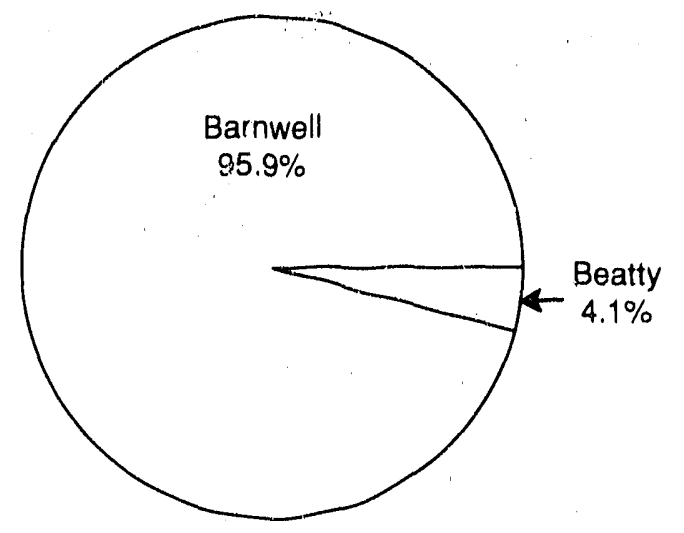

Direct Shipment

Waste Class

A
B
C

Total

Non-Direct Shipments

Grand Total

Total Direct Shipment

Activity (curies)

Total Non-Direct Shipment

Activity (curies)

Grand Total (curies)
Volume

$$
\begin{array}{r}
\multicolumn{1}{c}{\left(\mathrm{ft}^{3}\right)} \\
6,515.50 \\
0.00 \\
0.00 \\
\hline 6,515.50 \\
4,479.97 \\
\hline 10,995.47
\end{array}
$$

200.46

21.99

222.45

ACTIVITY PERCENTAGE BY DISPOSAL SITE (Percentages <.1\% are not displayed)

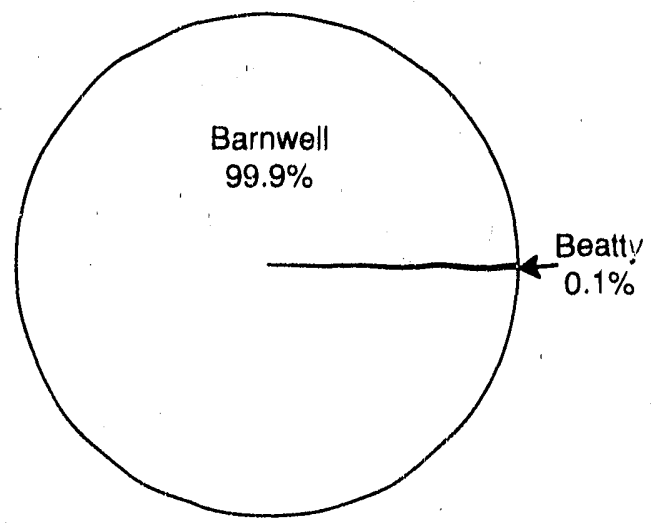

Volume Percentage by Waste Class for Direct Shipments and Total Non-Direct Volume Percentage

(Percentages <. $1 \%$ are not displayed)

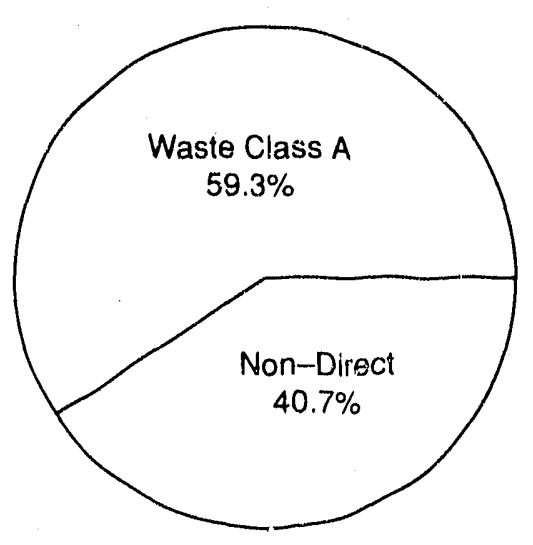




\section{NORTH CAROLINA \\ Low-Level Radioactive Wastes Received at Commercial Disposal Sites}

\begin{tabular}{l}
$\begin{array}{l}\text { Generator } \\
\text { Category }\end{array}$ \\
\hline Academic \\
Government \\
Industrial \\
Medical \\
Utility
\end{tabular}

Total

$\begin{array}{r}\begin{array}{c}\text { Volume } \\ \left(\mathrm{ft}^{3}\right)\end{array} \\ \hline 4,857.49 \\ 167.85 \\ 11,099.37 \\ 189.20 \\ 42,107.58 \\ \hline 58,421.49\end{array}$

Activity

(curies)

478.07

233.04

125.99

0.06

$6,552.92$

$7,390.08$
VOLUME PERCENTAGE BY SOURCE (Percentages <. $1 \%$ are not displayed)

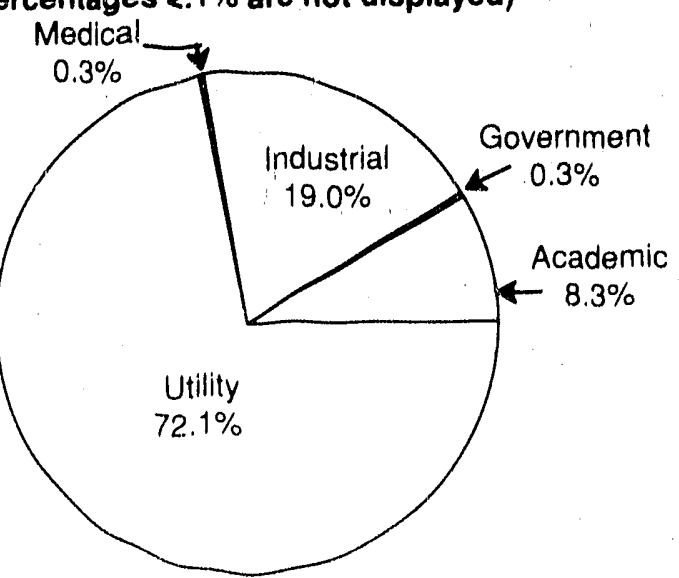

ACTIVITY PERCENTAGE BY SOURCE

(Percentages <.1\% are not dlsplayed)

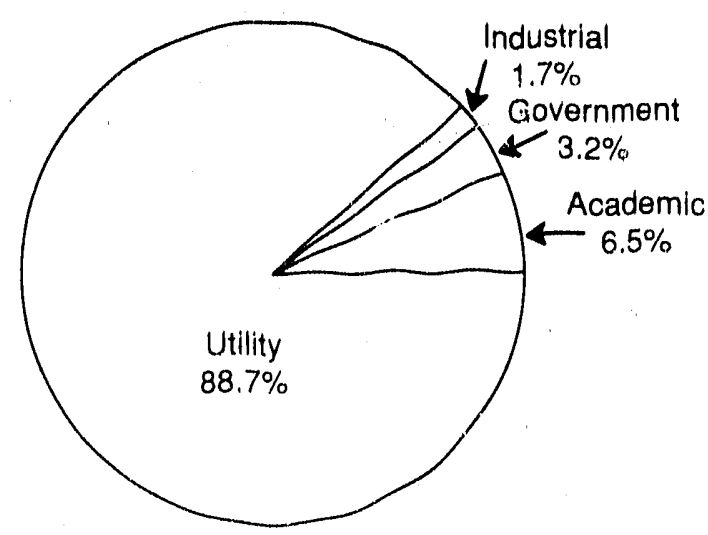




\section{NORTH CAROLINA \\ Low-Level Radioactive Wastes Received at Commercial Disposal Sites}

\begin{tabular}{c} 
Disposal \\
Site \\
\hline
\end{tabular}

Barnwell

Beatty

Richland

Total

$\begin{array}{r}\begin{array}{c}\text { Volume } \\ \left(\mathrm{ft}^{3}\right)\end{array} \\ \hline 57.607 .56 \\ 48.93 \\ 765.00 \\ \hline\end{array}$

$58,421.49$
Activity (curies)

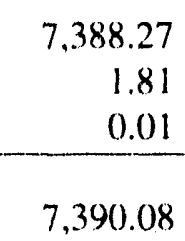

VOLUME PERCENTAGE BY DISPOSAL SITE (Percentages <. $1 \%$ are not displayed)

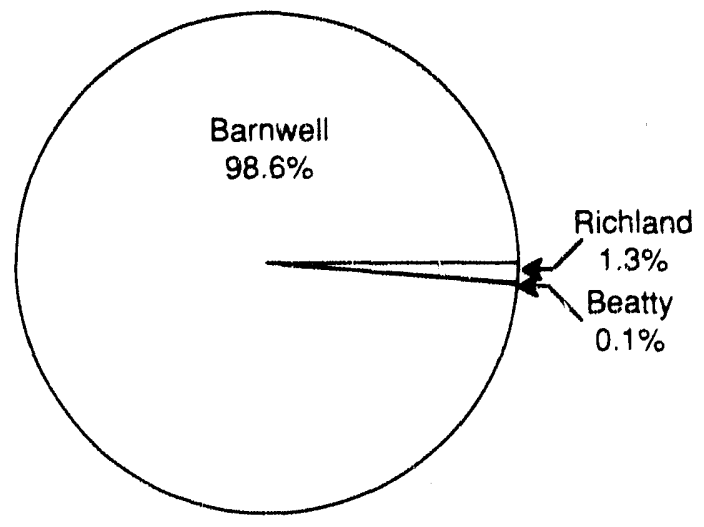

ACTIVITY PERCENTAGE BY DISPOSAL SITE (Percentages <.1\% are not displayed)

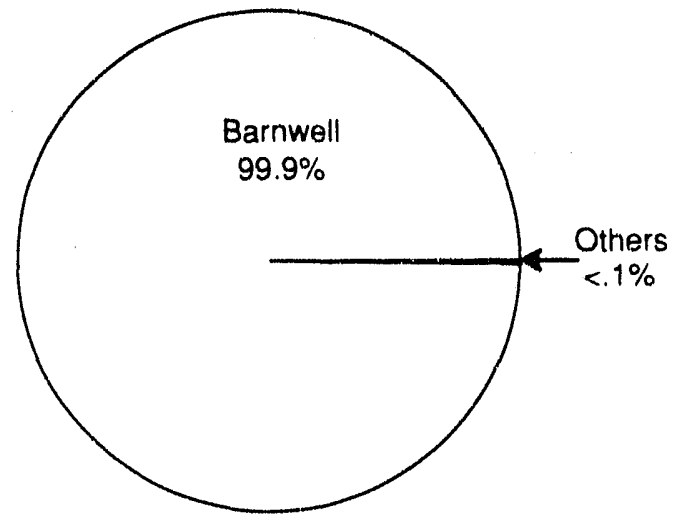

Volume Percentage by Waste Class for Direct Shipments and Total Non-Direct Volume Percentage (Percentages <. $1 \%$ are not displayed)
Direct Shipment

Waste Class

A

B

C

Total

Non-Direct Shipments

Grand Total

Total Direct Shipment

Activity (curies)

Total Non-Direct Shipment

Activity (curies)

Grand Total (curies)
Volume $\left(\mathrm{ft}^{3}{ }^{3}\right)$

$32,139.60$

$1,381.70$

500.80

$34,022.10$

$24,399.39$

58.421 .49

$6,551.81$

838.27

$7,390.08$

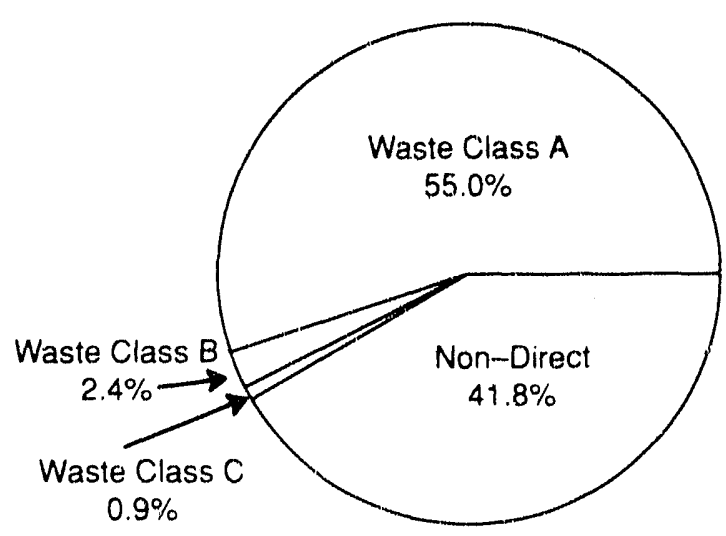

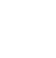

( 


\section{SOUTH CAROLINA \\ Low-Level Radioactive Wastes Received at Commercial Disposal Sites}

\begin{tabular}{l}
$\begin{array}{l}\text { Generator } \\
\text { Category }\end{array}$ \\
\hline Academic \\
Government \\
Industrial \\
Utility
\end{tabular}

Total

\begin{tabular}{c}
$\begin{array}{c}\text { Volume } \\
\left(\mathrm{ft}^{3}\right)\end{array}$ \\
\hline $1,442.00$ \\
$10,289.60$ \\
$53,291.62$ \\
$31,672.15$ \\
\hline $96,695.37$
\end{tabular}

Activity

(curies)

2.35

21.80

69.18

$2,337.66$

$2,430.99$
VOLUME PERCENTAGE BY SOURCE (Percentages <.1\% are not displayed)

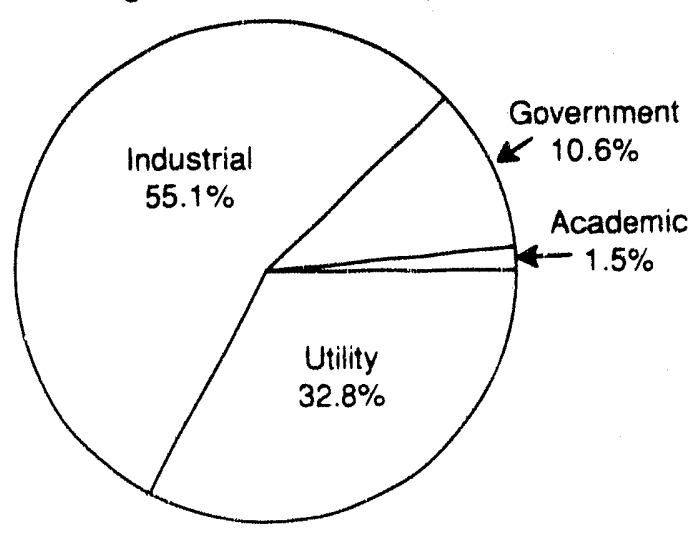

ACTIVITY PERCENTAGE BY SOURCE

(Percentages <.1\% are not displayed)

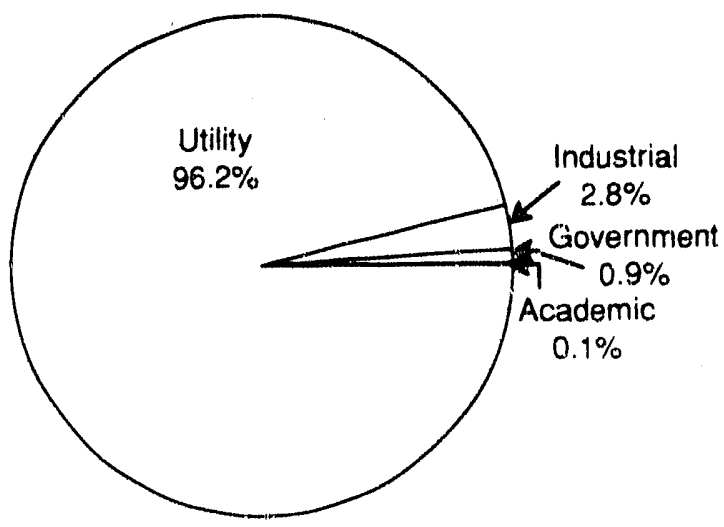




\section{SOUTH CAROLINA \\ Low-Level Radioactive Wastes Received at Commercial Disposal Sites}

\begin{tabular}{c} 
Disposal \\
Site \\
\hline
\end{tabular}

Barnwell

Kichland

Total

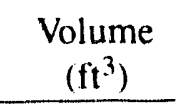

$96,687.87$

7.50

$96,695.37$
Activity

(curies)

$$
\begin{gathered}
\begin{array}{c}
2,430.99 \\
<1
\end{array} \\
\hline 2,430.99
\end{gathered}
$$

VOLUME PERCENTAGE BY DISPOSAL SITE (Percentages <. $1 \%$ are not displayed)

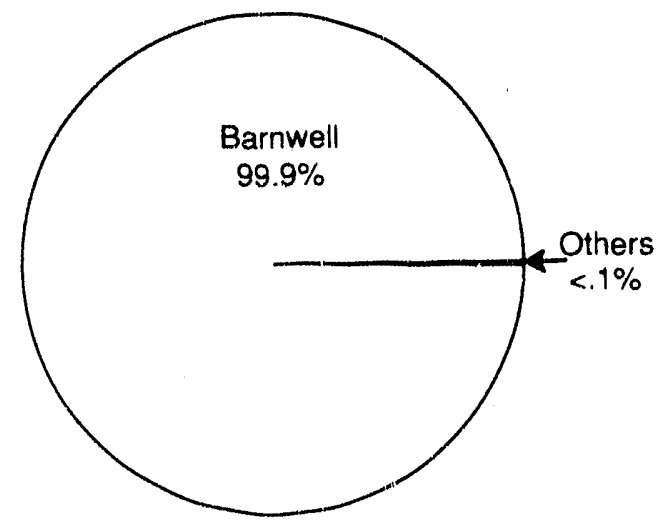

Direct Shipment

Waste Class

A

B

C

Total

Non-Direct Shipments

Grand Total

Total Direct Shipment

Activity (curies)

Total Non-Direct Shipment

Activity (curies)

Grand Total (curies)
Volume

$\left(\mathrm{ft}^{3}\right)$

$52,293.38$

$2,587.30$

947.50

$55,828.18$

$40,867.19$

$96,695.37$

$2,371.12$

59.86

2.430 .99
ACTIVITY PERCENTAGE BY DISPOSAL SITE

(Percentages <.1\% are not displayed)

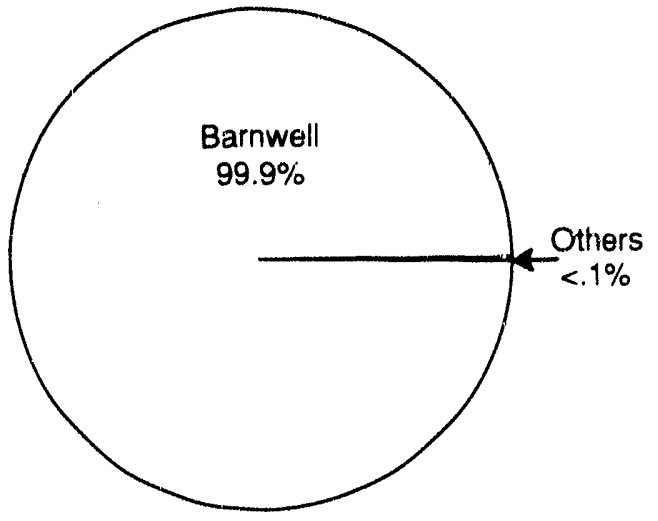

Volume Percentage by Waste Class for Direct Shipments and Total Non-Direct Volume Percentage

(Percentages <. $1 \%$ are not displayed)

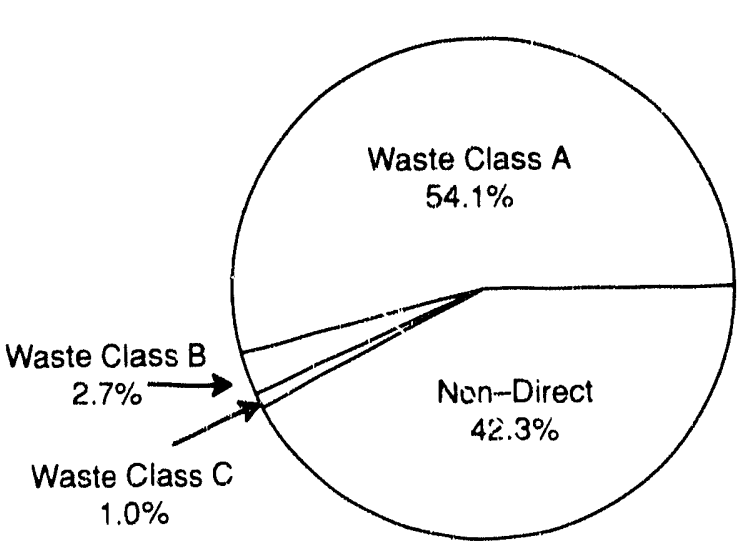




\section{TENNESSEE \\ Low-Level Radioactive Wastes Received at Commercial Disposal Sites}

\begin{tabular}{l}
$\begin{array}{l}\text { Generator } \\
\text { Category }\end{array}$ \\
\hline Academic \\
Government \\
Industrial \\
Medical \\
Utility
\end{tabular}

Total

$$
\begin{array}{r}
\begin{array}{c}
\text { Volume } \\
\left(\mathrm{ft}^{3}\right)
\end{array} \\
\hline 1,748.15 \\
3,857.20 \\
96,691.49 \\
218.39 \\
16,441.90 \\
\hline
\end{array}
$$

118.957 .13
Activity

(curies)

$\begin{array}{r}1.52 \\ 0.40 \\ 490.05 \\ 0.67 \\ 2.550 .95 \\ \hline 3,043.58\end{array}$

VOLUME PERCENTAGE BY SOURCE (Percentages <. $1 \%$ are not displayed)

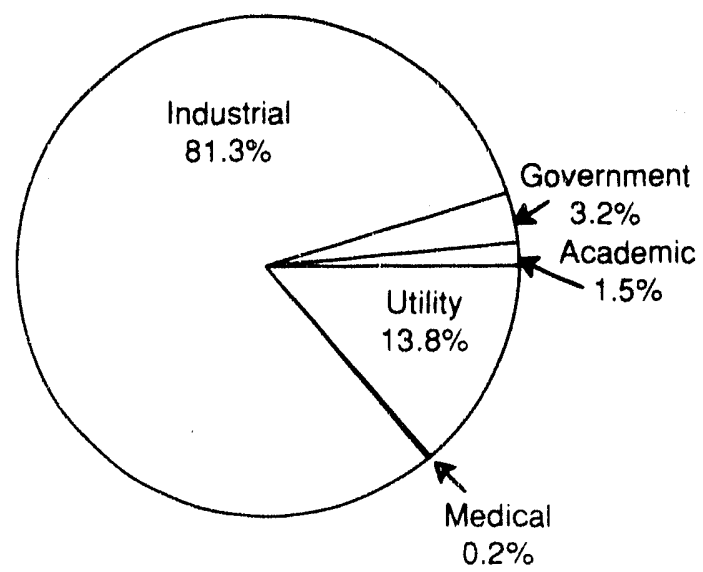

ACTIVITY PERCENTAGE BY SOURCE

(Percentages <. $1 \%$ are not displayed)

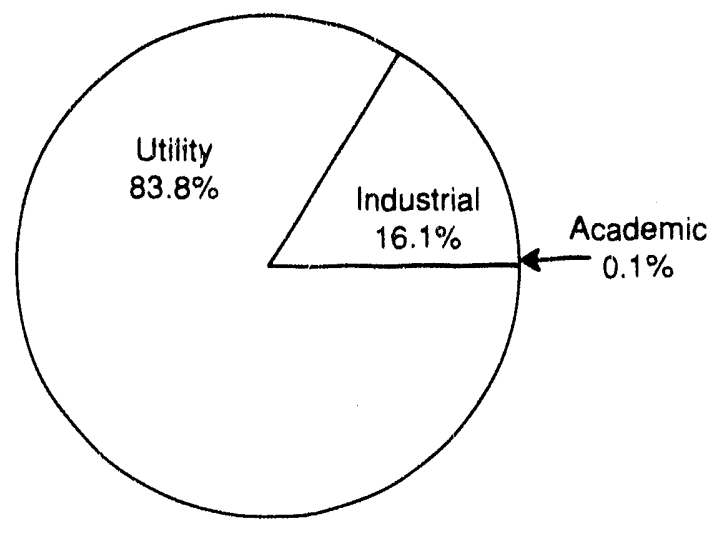




\section{TENNESSEE \\ Low-Level Radioactive Wastes Received at Commercial Disposal Sites}

\begin{tabular}{c} 
Disposal \\
Site \\
\hline
\end{tabular}

Barnwell

Beatty

Richland

Total

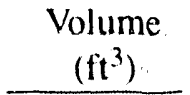

117.549 .84

8.39

$1,398.90$

$118,957.13$
Activity

(curies)

$3,042.93$

0.57

0.09

$3,043.58$
VOLUME PERCENTAGE BY SOURCE (Percentages <.1\% are not displayed)

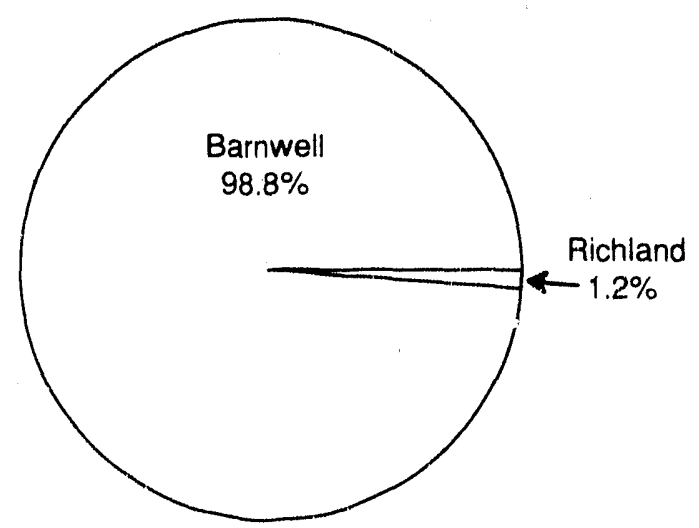

Direct Shipment

Waste Class

\section{A}

B

$\mathrm{C}$

Total

Non-Direct Shipments

Grand Total

Total Direct Shipment

Activity (curies)

Total Non-Direct Shipment

Activity (curies)

Grand Total (curies)
ACTIVITY PERCENTAGE BY DISPOSAL SITE

(Percentages <. $1 \%$ are not displayed)

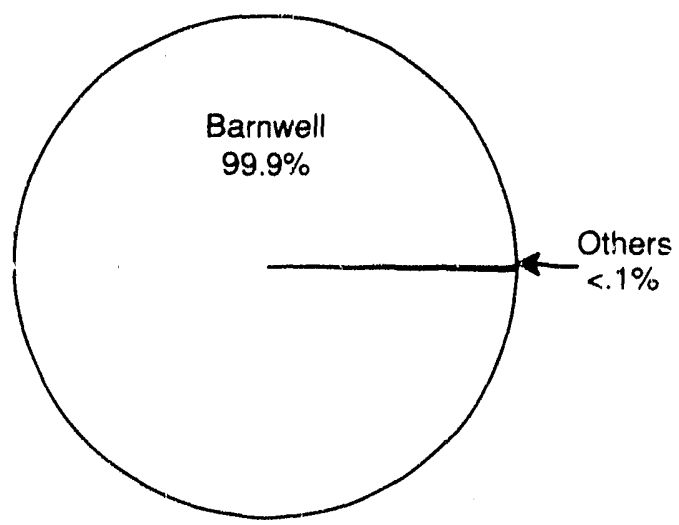

Volume Percentage by Waste Class for Direct Shipments and Total Non-Direct Volume Percentage

(Percentages <.1\% are not displayed)
Volume

$\left(\mathrm{ft}^{3}\right)$

$68,170.80$

120.30

639.70

68.930 .80

$50,026.33$

$118,957.13$

$3,015.24$

Waste Class C

$0.5 \%$

28.34

3.043 .58 
VIRGINIA

\section{Low-Level Radioactive Wastes Received at Commercial Disposal Sites}

\begin{tabular}{l}
\multicolumn{1}{c}{$\begin{array}{l}\text { Generator } \\
\text { Category }\end{array}$} \\
\hline Academic \\
Government \\
Industrial \\
Medical \\
Utility \\
Total
\end{tabular}

VOLUME PERCENTAGE BY SOURCE (Percentages <.1\% are not displayed)

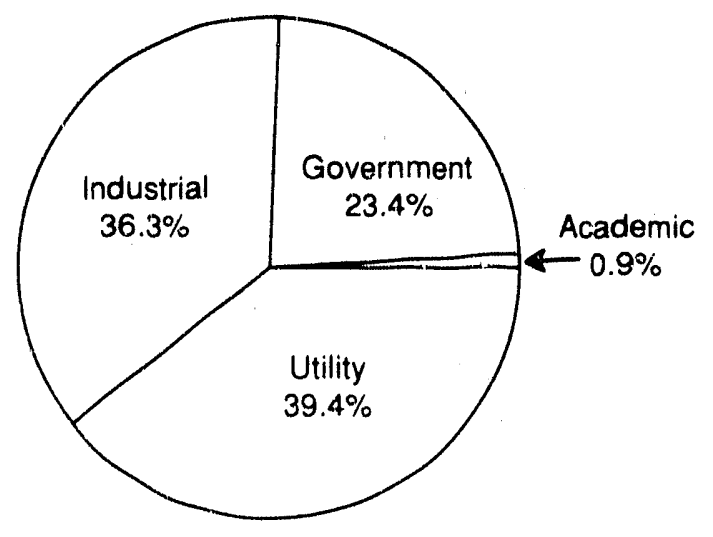

Volume

$\left(\mathrm{ft}^{3}\right.$ )

737.32

$19,408.00$

$30,056.95$

19.03

$32,613.00$

$82,834.30$
Activity

(curies)

0.78

21.25

8.93

0.02

3.022 .54

$3,053.52$
ACTIVITY PERCENTAGE BY SOURCE

(Percentages <.1\% are not displayed)

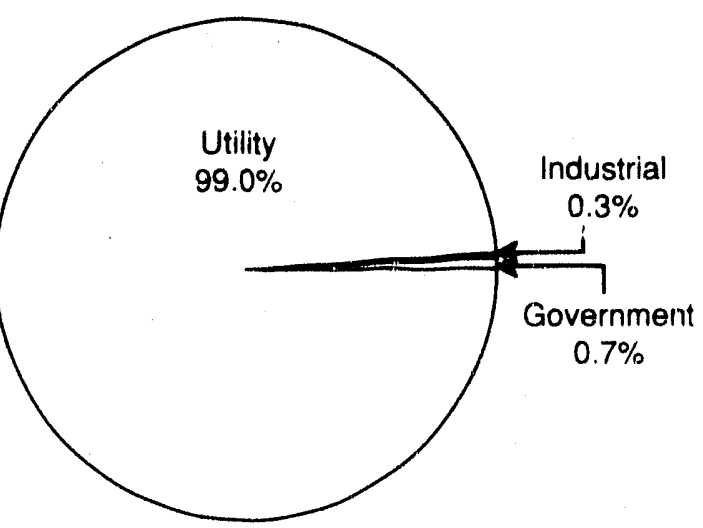




\section{VIRGINIA \\ Low-Level Radioactive Wastes Received at Commercial Disposal Sites}

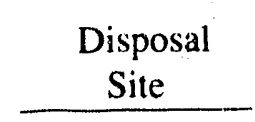

Barnwell

Beatty

Richland

Total

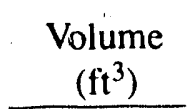

$82,592.22$

21.28

220.80

$82,834.30$
Activity

(curies)

$3,052.37$

0.03

1.12

$3,053.52$
VOLUME PERCENTAGE BY DISPOSAL SITE

(Percentages <. $1 \%$ are not displayed)

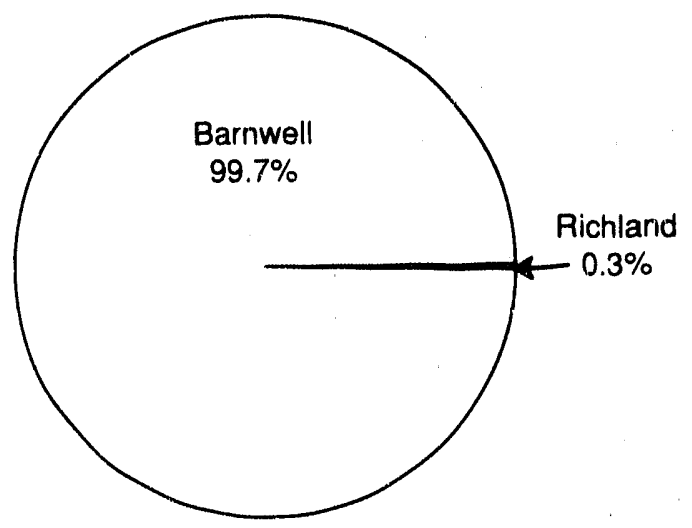

Direct Shipment

Waste Class

A

B

C

Total

Non-Direct Shipments

Grand Total

Total Direct Shipment

Activity (curies)

Total Non-Direct Shipment

Activity (curies)

Grand Total (curies)
Volume

$\left(\mathrm{ft}^{3}\right)$

$49,092.12$

1.629 .00

667.30

$51,388.42$

$31,445.88$

$82,834.30$

$3,007.64$

45.88

$3,053.52$
ACTIVITY PERCENTAGE BY DISPOSAL SITE (Percentcges <.1\% are not displayed)

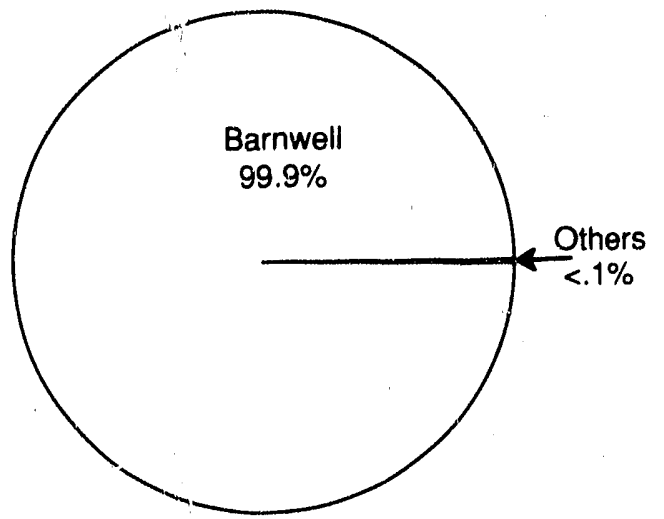

Volume Percentage by Waste Class for Direct Shipments and Total Non-Direct Volume Percentage (Percentages <.1\% are not displayed) 


\section{SOUTHWEST COMPACT Low-Level Radioactive Wastes Received at Commercial Disposal Sites}

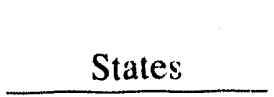

Arizona

California

North Dakota

South Dakota

Total

\begin{tabular}{c}
$\begin{array}{c}\text { Volume } \\
\left(\mathrm{ft}^{3}\right)\end{array}$ \\
\hline
\end{tabular}

$31,756.16$

$117,965.93$

105.20

0.60

$149,827.89$
Activity

(curies)

$1,123.27$

$9,949.05$

1.84

0.50

$11,074.66$

VOLUME PERCENTAGE BY STATE

(Percentages <.1\% are not displayed)

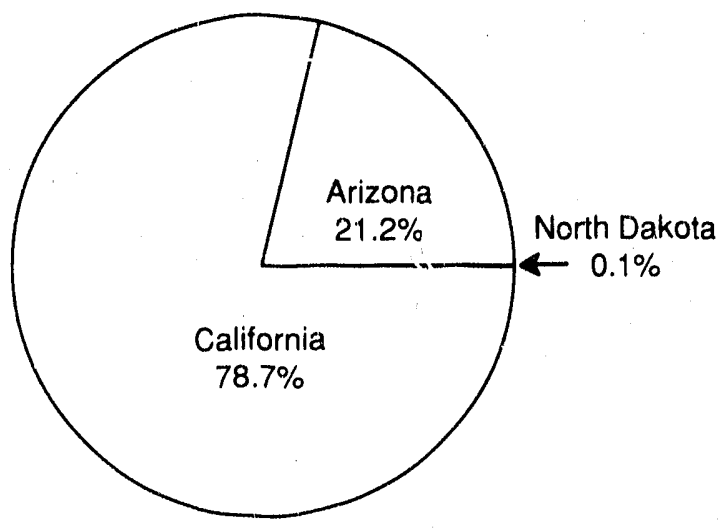

VOLUME PERCENTAGE BY CATEGOAY

(Percentages <.1\% are not displayed)

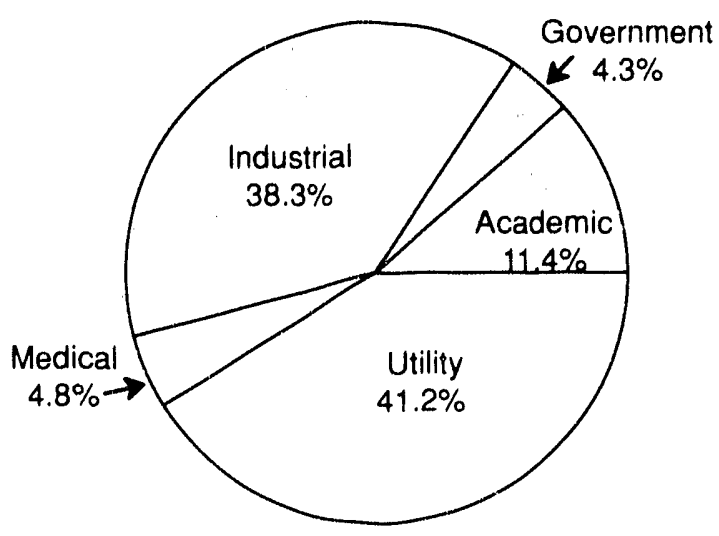




\section{ARIZONA \\ Low-Level Radioactive Wastes Received at Commercial Disposal Sites}

$\begin{array}{lrr}\begin{array}{l}\text { Generator } \\ \text { Category }\end{array} & \begin{array}{c}\text { Volume } \\ \left(\mathrm{ft}^{3}\right)\end{array} & \begin{array}{c}\text { Activity } \\ \text { (curies) }\end{array} \\ \text { Academic } & 846.66 & 4.57 \\ \text { Government } & 102.00 & 411.19 \\ \text { Industrial } & 105.45 & 0.45 \\ \text { Medical } & 7.80 & <1 \\ \text { Utility } & 30,694.25 & 707.06 \\ & & \\ \text { Total } & 31,756.10 & 1,123.27\end{array}$

VOLUME PERCENTAGE BY DISPOSAL SITE

(Percentages <.1\% are not dt. splayed)

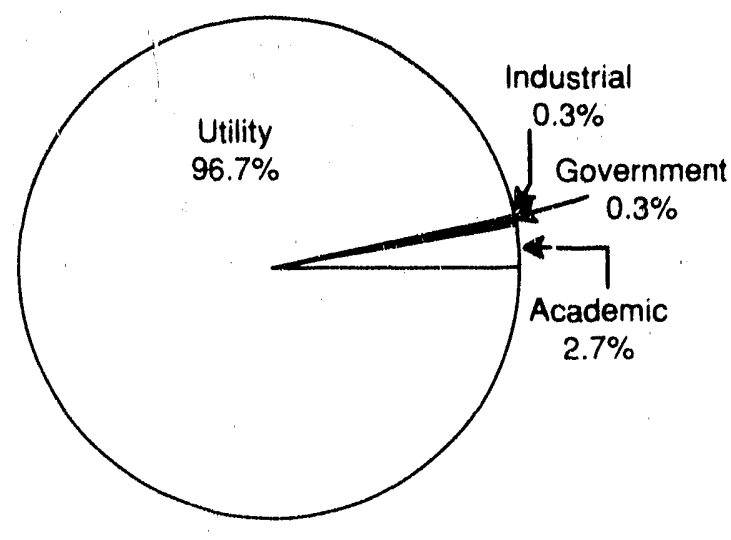

ACTIVITY PERCENTAGE BY SOURCE

(Percentages <.1\% are not displayed)

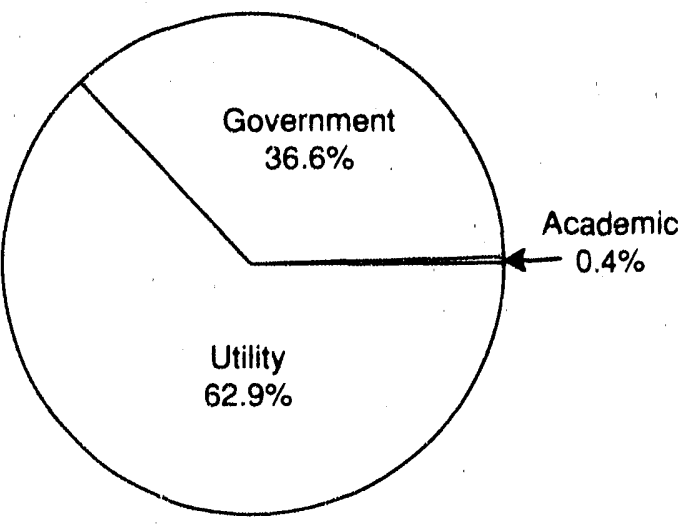




\section{ARIZONA \\ Low-Level Radioactive Wastes Received at Commercial Disposal Sites}

Disposal
Site

Barnwell

Beatty

Richland

Total

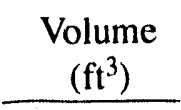

94.20

$3,354.08$

$28,307.88$

$31,756.16$
Activity

(curies)

411.19

10.03

702.05

$1,123.27$
VOLUME PERCENTAGE BY DISPOSAL SITE

(Percentages <.1\% are not displayed)

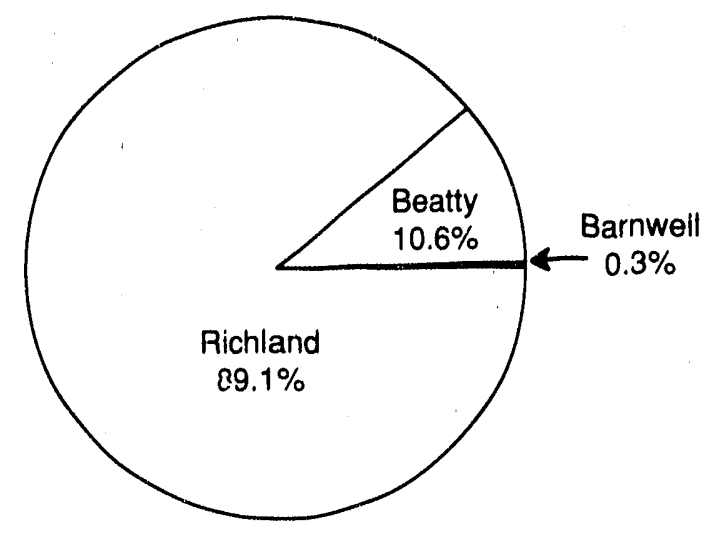

Direct Shipment

Waste Class

A
B
C
Total

Volume

$\left(\mathrm{Et}^{3}\right)$

Non-Direct Shipments

Grand Total

Total Direct Shipment

Activity (curies)

Total Non-Direct Shipment

Activity (curies)

Grand Total (curies)
423.20

$27,304.60$

492.20

180.70

$27,977.50$

$\underline{3,778.66}$

$31,756.16$

$1,123.27$
ACTIVITY PERCENTAGE BY DISPOSAL SITE (Percentages <. $1 \%$ are not displayed)

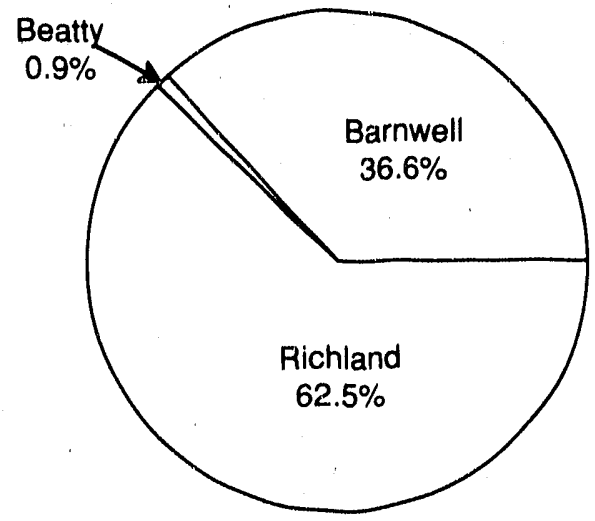

Volume Percentage by Waste Class for Direct Shipments and Total Non-Direct Volume Percentage (Percentages <. $1 \%$ are not displayed)

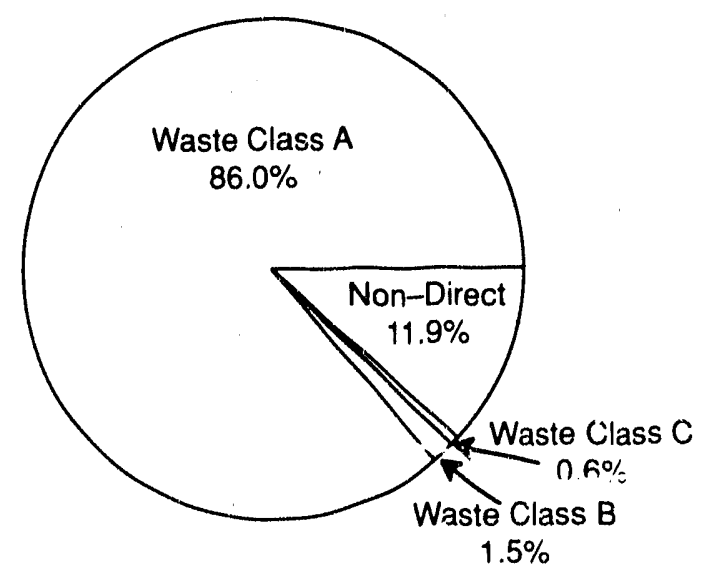




\section{CALIFORNIA \\ Low-Level Radioactive Wastes Received at Commercial Disposal Sites}

\author{
Generator \\ Category \\ Academic \\ Government \\ Industrial \\ Medical \\ Utility
}

Total

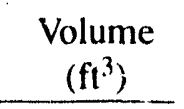

$16,174.35$

$6,361.15$

$57,223.09$

$7,206.24$

$31,001.10$

$117,965.93$
Activity

(curies)

$$
87.86
$$

$2,125.77$

$2,478.98$

48.71

$5,207.72$

$9,949.05$
VOLUME PERCENTAGE BY SOURCE

(Percentages <.1\% are not displayed)

Government

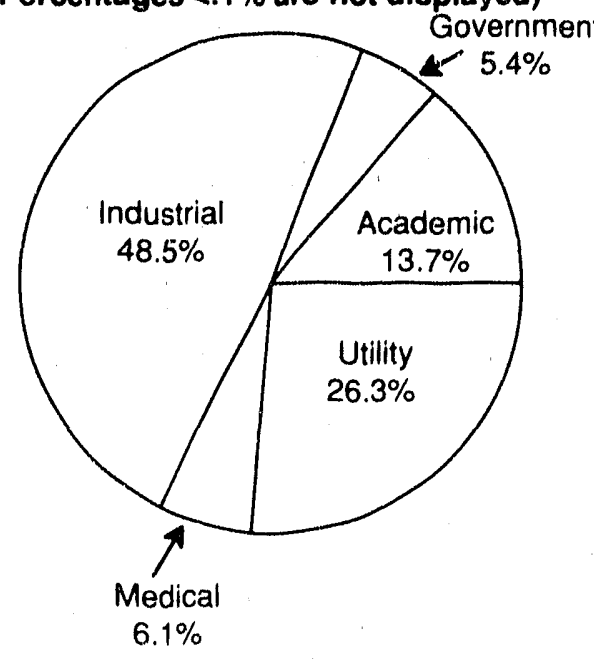

ACTIVITY PERCENTAGE BY SOURCE

(Percentages <.1\% are not displayed)

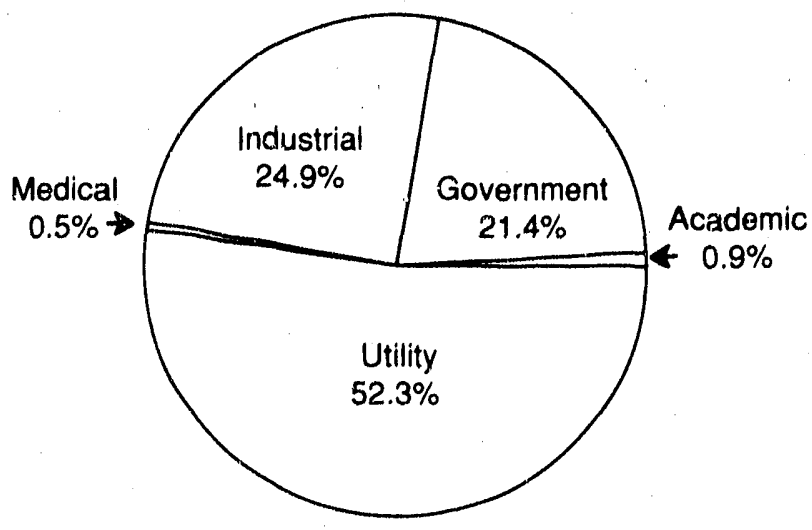




\section{CALIFORNIA \\ Low-Level Radioactive Wastes Received at Commercial Disposal Sites}

\begin{tabular}{c}
$\begin{array}{c}\text { Disposal } \\
\text { Site }\end{array}$ \\
\hline
\end{tabular}

Barnwell

Beátty

Richland

Total

\author{
Volumie \\ $\left(\mathrm{ft}^{3}\right)$
}

$5,351.40$

$55,454.72$

$57,159.81$

$117,965.93$
Activity

(curies)

$3,564.59$

786.06

$5,598.40$

$9,949.05$

\section{VOLUME PERCENTAGE RY DISPOSAL SITE (Percentages <. $1 \%$ are not displayed)}

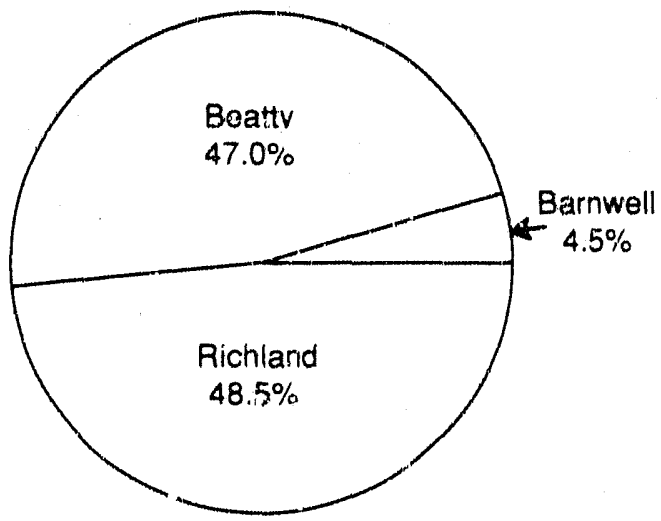

ACTIVITY PERCENTAGE BY DISPOSAL SITE (Percentageo <. $1 \%$ are not displayed)

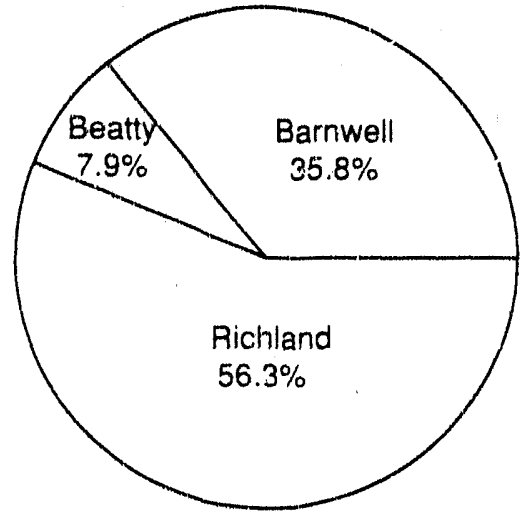

Volume Percentage by Waste Class for Direct Shipments and Total Non-Direct Volume Percentage (Percenteges <. $1 \%$ are not displayed)
Direct Shipment

Waste Class

A

B

C

Total

Non--Direct Shipments

Grand Total

Total Direct Shipment

Activity (curies)

Total Non-Direct Shipment

Activity (curies)

Grand Total (curies)
Volume

$\left(\mathrm{ft}^{3}\right)$

$61,559.04$

$3,225.70$

1.423 .20

$66,207.94$

51.757 .99

$1 j \div 965.93$

5.456 .71

4.492 .34

9.949 .05 


\section{NORTH DAKOTA \\ Low-Level Radioactive Wastes Received at Commercial Disposal Sites}

\begin{tabular}{l} 
Generator \\
Category \\
\hline
\end{tabular}

Academic

Government

Industrial

Total

\begin{tabular}{c}
$\begin{array}{c}\text { Volume } \\
\left(\mathrm{ft}^{3}\right)\end{array}$ \\
\hline
\end{tabular}

29.50

0.70

75.00

105.20
Activity

(curies)

0.01

1.11

0.72

1.84
VOLUME PERCENTAGE BY SOURCE (Percenteges <. $1 \%$ are not displayed)

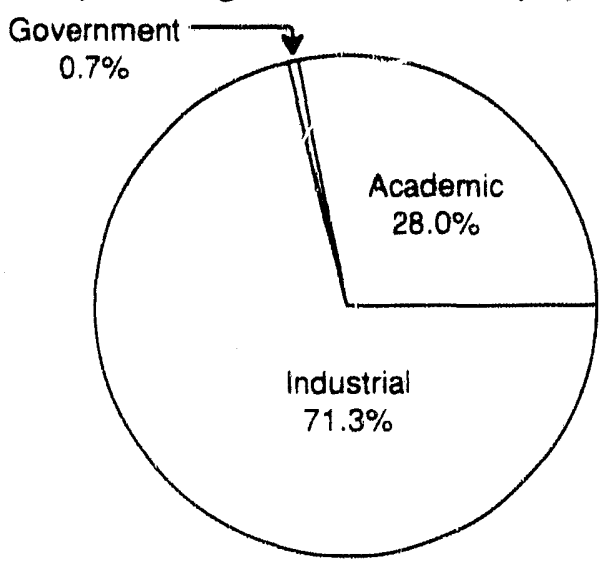

ACTIVITY PERCENTAGE BY SOURCE

(Percentages <.1\% are not displayed)

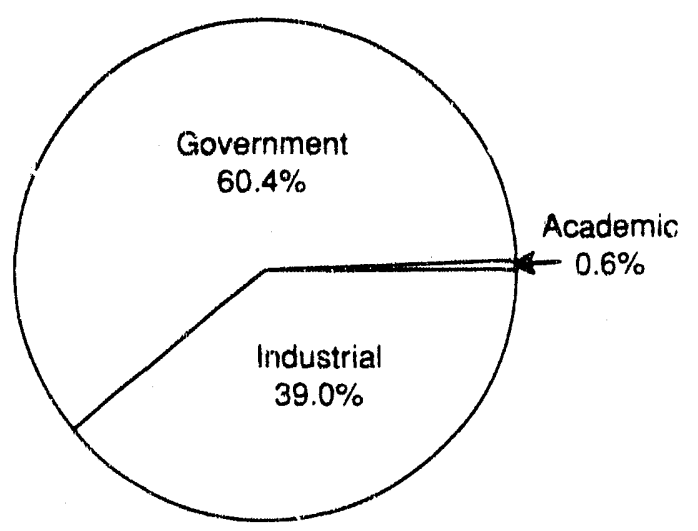




\title{
NORTH DAKOTA \\ Low-Level Radioactive Wastes Received at Commercial Disposal Sites
}

Disposal
Site

Barnwell

Beauly

Richland

Total
Volume

$\left(\mathrm{ft}^{3}\right)$

$\begin{array}{r}0.70 \\ 9.20 \\ 95.30 \\ \hline\end{array}$

105.20
Activity

(curies)

1.11

0.69

0.04

1.84
VOLUME PERCENTAGE BY DISPOSAL SITE (Percentages <.1\% are not displayed)

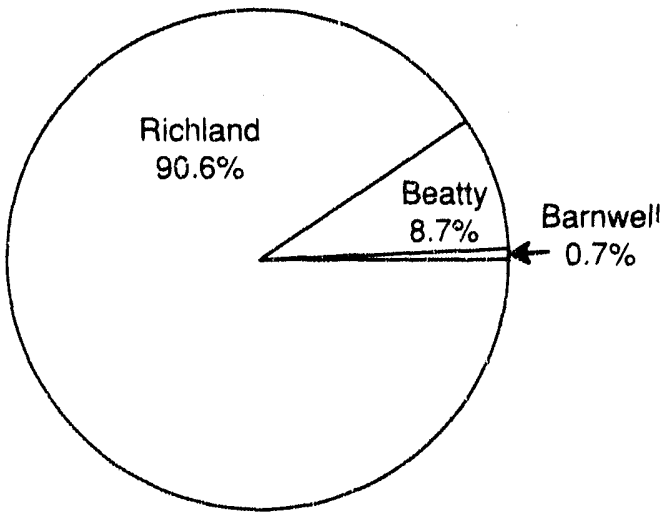

ACTIVITY PERCENTAGE BY DISPOSAL SITE

(Percentages <. $1 \%$ are not displayed)

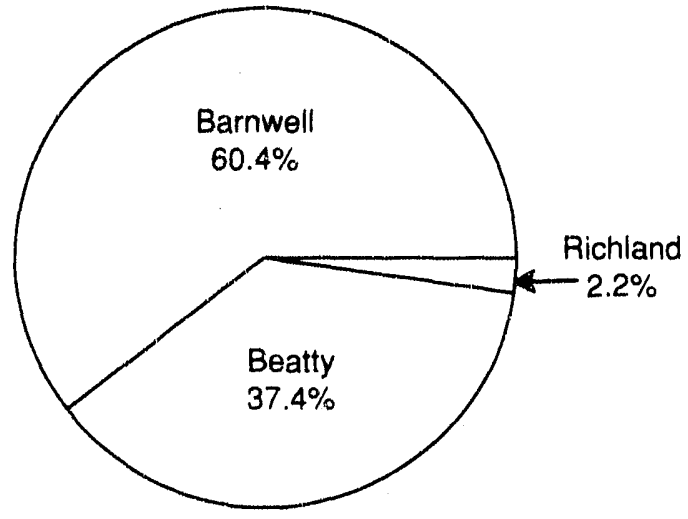

\begin{abstract}
Volume Percentage by Waste Class for Direct Shipments and Total Non-Direct Volume Percentage

(Percentages <. $1 \%$ are not displayed)
\end{abstract}

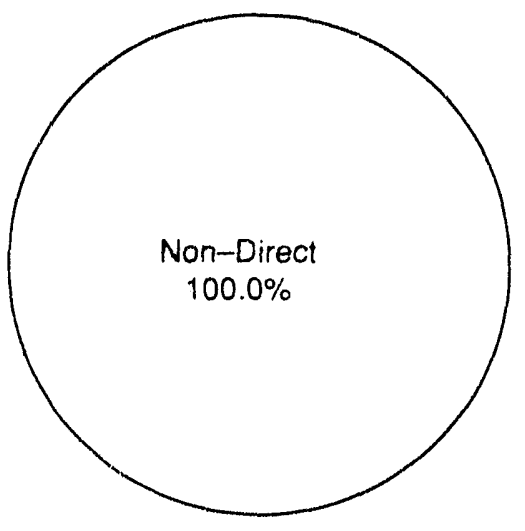


SOUTH DAKOTA

\section{Low-Level Radioactive Wastes Received at Commercial Disposal Sites}

\begin{tabular}{l} 
Generator \\
Category \\
\hline
\end{tabular}

Government

Total
Volume

$\left(\mathrm{ft}^{3}\right)$

$\frac{0.60}{0.60}$

0.60
Activity

(curies)

$$
-\frac{0.50}{0.50}
$$

VOLUME PERCENTAGE BY SOURCE (Percentages <. $1 \%$ are not displayed)

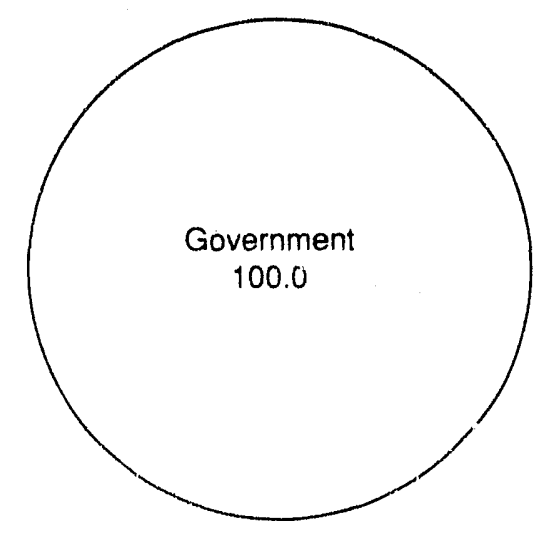

ACTIVITY PERCENTAGE BY SOURCE

(Percentages <. $1 \%$ are not displayed)

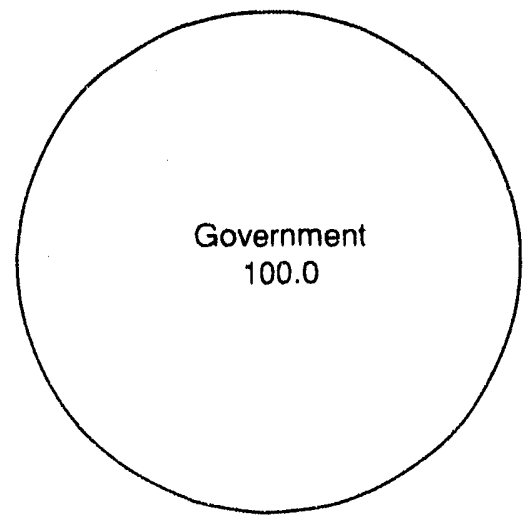




\section{SOUTH DAKOTA \\ Low-Level Radioactive Wastes Received at Commercial Disposal Sites}

\begin{tabular}{lrr}
$\begin{array}{c}\text { Disposal } \\
\text { Site }\end{array}$ & $\begin{array}{c}\text { Volume } \\
\left(\mathrm{ft}^{3}\right)\end{array}$ & $\begin{array}{l}\text { Activity } \\
\text { (curies) }\end{array}$ \\
\cline { 1 - 1 } Barnwell & 0.60 & 0.50 \\
Total & 0.60 & 0.50
\end{tabular}

VOLUME PERCENTAGE BY DISPOSAL SITE

(Percentages <. $1 \%$ are not displayed)

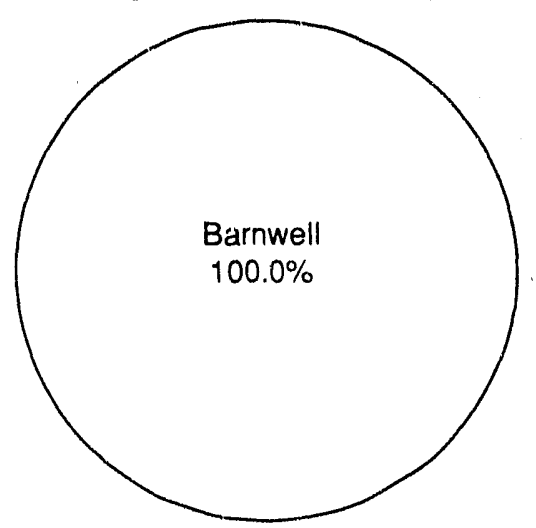

ACTIVITY PERCENTAGE BY DISPOSAL SITE

(Percentages <. $1 \%$ are not displayed)

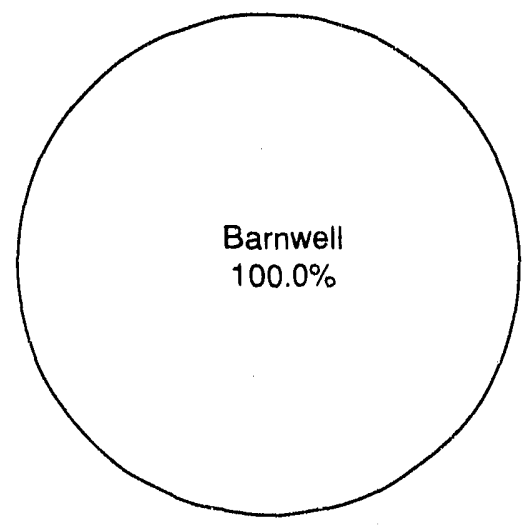

Volume Percentage by Waste Class for Direct Shipments and Total Non-Direct Volume Percentage

(Percentages <.1\% are not displayed)

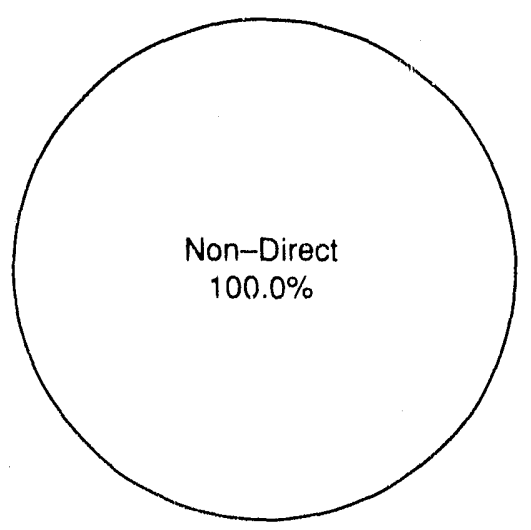




\section{UNAFFILIATED \\ Low-Level Radioactive Wastes Received at Commercial Disposal Sites}

States

Army Out U.S.

Dist. of Columbia

Maine

Massachusetts

New Hampshire

New York

Rhode Island

Texas

Vermont

Total \begin{tabular}{c}
$\begin{array}{c}\text { Volume } \\
\left(\mathrm{ft}^{3}\right)\end{array}$ \\
\hline
\end{tabular}

164.30

925.09

$15,633.40$

$56,527.07$

26.70

$96,893.38$

$1,419.31$

$22,157.48$

173.20

$193,919.93$
Activity

(curies)

192.92

0.69

273.78

$57,385.70$

26.92

$101,453.86$

22.70

$11,357.03$

2.15

$170,715.74$ 


\section{ARMY OUT U.S. \\ Low-Level Radioactive Wastes Received at Commercial Disposal Sites}

\section{Generator \\ Category}

Government

Total

\begin{tabular}{c}
$\begin{array}{c}\text { Volume } \\
\left(\mathrm{ft}^{3}\right)\end{array}$ \\
\hline
\end{tabular}

164.30

164.30
Activity

(curies)

192.92

192.92
VOLUME PERCENTAGE BY SOURCE (Percentages <.1\% are not displayed)

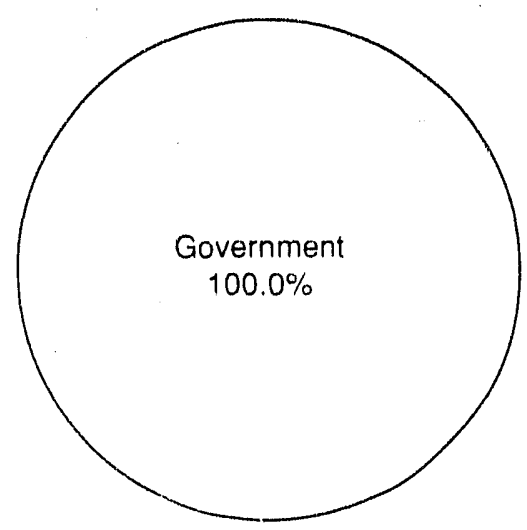

ACTIVITY PERCENTAGE BY SOURCE

(Percentages $<.1 \%$ are not displayed)

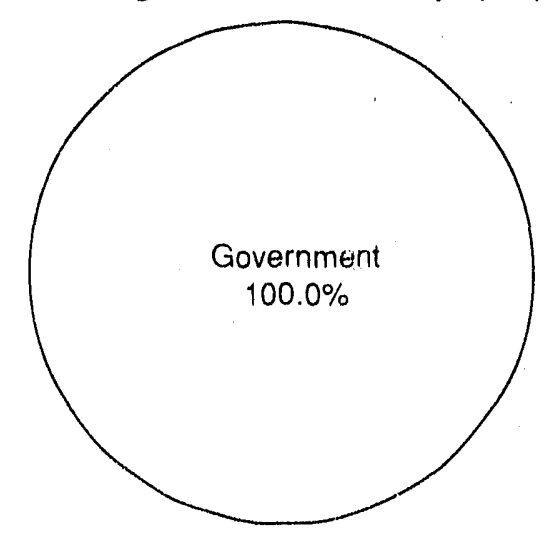




\section{ARMY OUT U.S. \\ Low-Level Radioactive Wastes Received at Commercial Disposal Sites}

\begin{tabular}{c}
$\begin{array}{c}\text { Disposal } \\
\text { Site }\end{array}$ \\
\hline
\end{tabular}

Barnwell

Total

\author{
Volume \\ $\left(\mathrm{ft}^{3}\right)$
}

164.30

164.30
Activity

(curies)

192.92

192.92
VOLUME PERCENTAGE BY DISPOSAL SITE

(Percentages $<.1 \%$ are not displayed)

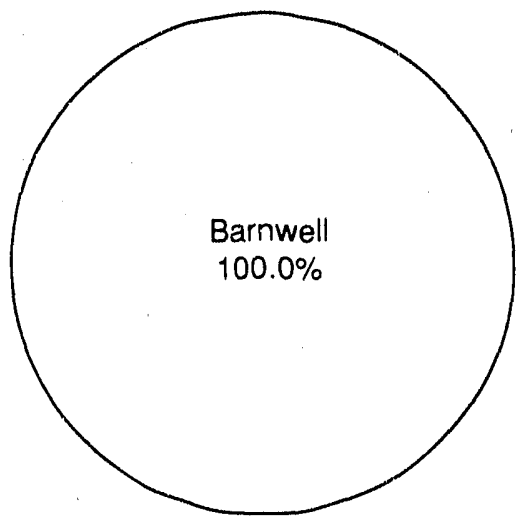

Direct Shipment

Waste Class

A
B
C

Total

Non-Direct Shipments

Grand Total

Total Direct Shipment

Activity (curies)

Total Non-Direct Shipment

Activity (curies)

Grand Total (curies)
Volume

$\left(\mathrm{ft}^{3}\right)$

150.00

0.00

0.00

150.00

14.30

164.30

0.00

192.92

192.92
ACTIVITY PERCENTAGE BY DISPOSAL SITE

(Percentages <.1\% are not displayed)

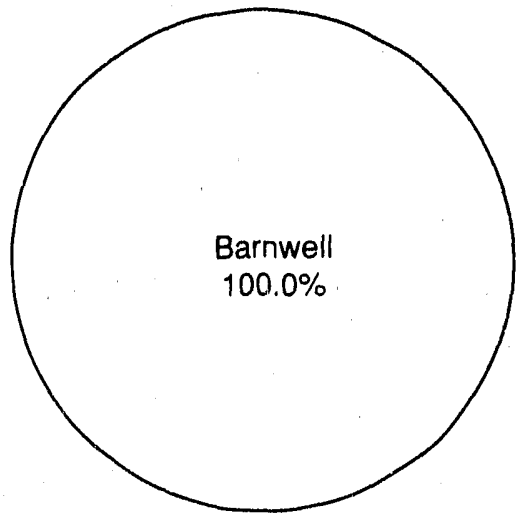

Volume Percentage by Waste Class

for Direct Shipments and Total

Non-Direct Volume Percentage

(Percentages <. $1 \%$ are not displayed)

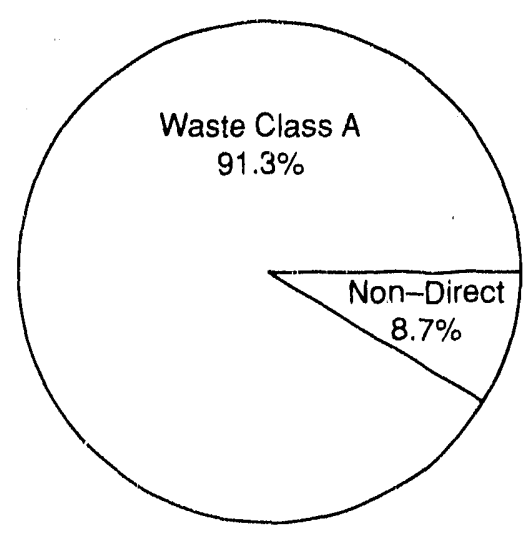




\section{DISTRICT OF COLUMBIA \\ Low-Level Radioactive Wastes Received at Commercial Disposal Sites}

\begin{tabular}{lrc}
$\begin{array}{c}\text { Generator } \\
\text { Category }\end{array}$ & \multicolumn{1}{c}{$\begin{array}{c}\text { Volume } \\
\left(\mathrm{ft}^{3}\right)\end{array}$} & $\begin{array}{c}\text { Activity } \\
(\text { curies })\end{array}$ \\
\cline { 2 - 3 } Academic & 88.95 & 0.05 \\
Government & 359.34 & 0.17 \\
Industrial & 1.04 & $<1$ \\
Medical & 475.22 & 0.47 \\
Utility & 0.54 & $<1$ \\
Total & 925.09 & 0.69
\end{tabular}

VOLUME PERCENTAGE BY SOURCE

ACTIVITY PERCENTAGE BY SOURCE

(Percentages <. $1 \%$ are not displayed)

(Percentages <. $1 \%$ are not displayed)
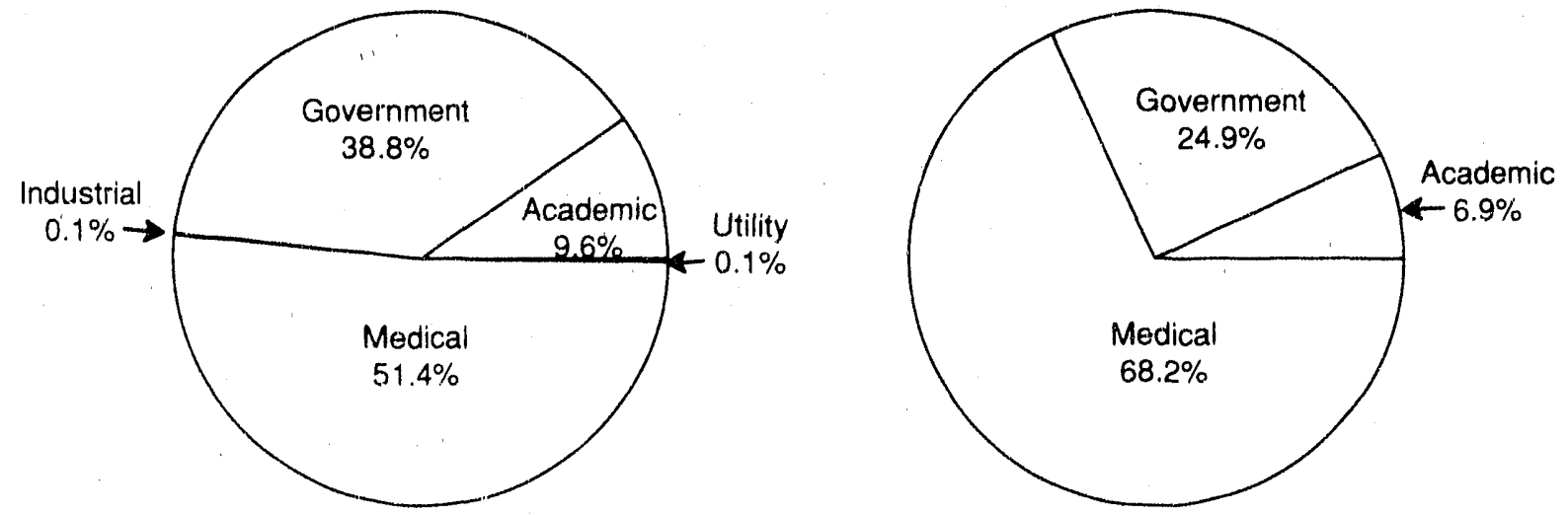


\section{DISTRICT OF COLUMBIA \\ Low-Level Radioactive Wastes Received \\ at Commercial Disposal Sites}

Disposal
Site

Barnwell

Beatty

Richland

Total

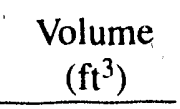

169.50

358.61

396.98

925.09
Activity

(curies)

0.07

0.46

0.16

0.69
VOLUME PERCENTAGE BY DISPOSAL SITE

(Percentages <. $1 \%$ are not displayed)

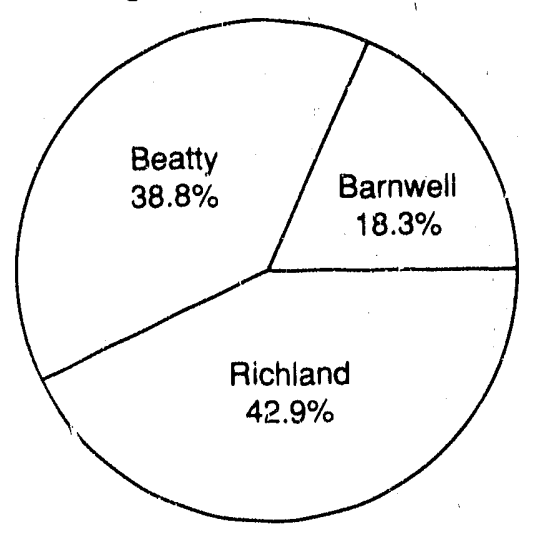

ACTIVITY PERCENTAGE BY DISPOSAL SITE

(Percentages <. $1 \%$ are not displayed)

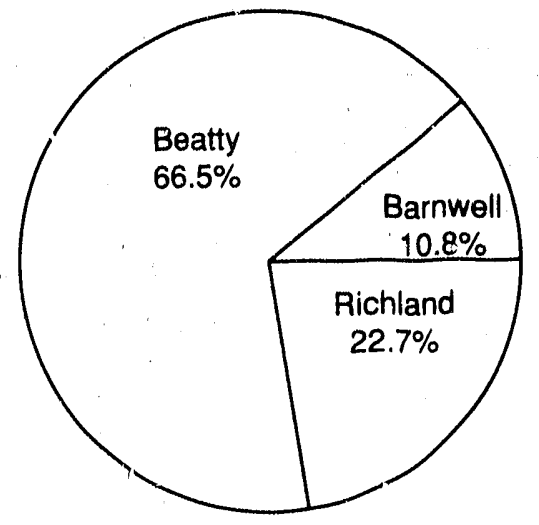

Volume Percentage by Waste Class for Direct Shipments and Total Non-Direct Volume Percentage (Percentages <.1\% are not displayed)

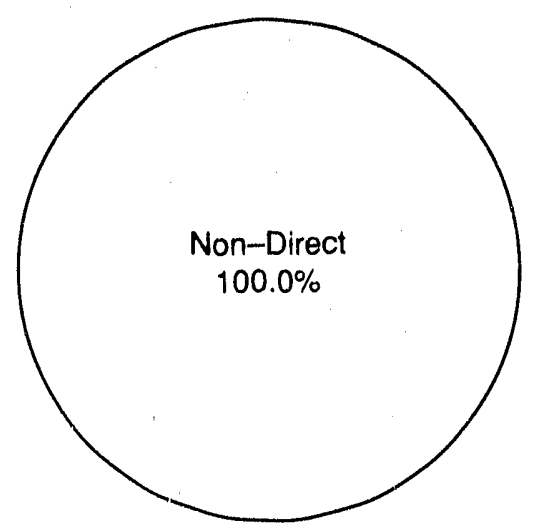




\section{MAINE \\ Low-Level Radioactive Wastes Received at Commercial Disposal Sites}

\begin{tabular}{lrr}
$\begin{array}{c}\text { Generator } \\
\text { Category }\end{array}$ & $\begin{array}{c}\text { Volume } \\
\left(\mathrm{ft}^{3}\right)\end{array}$ & \multicolumn{1}{c}{$\begin{array}{c}\text { Activity } \\
\text { (curies) }\end{array}$} \\
\cline { 2 - 3 } & $\begin{array}{r}129.93 \\
\text { Academic }\end{array}$ & 0.10 \\
Government & $8,437.40$ & 3.31 \\
Industrial & 68.47 & 0.24 \\
Utility & $6,997.60$ & 270.13 \\
Total & $15,633.40$ & 273.78
\end{tabular}

VOLUME PERCENTAGE BY SOURCE (Percentages $<.1 \%$ are not displayed)

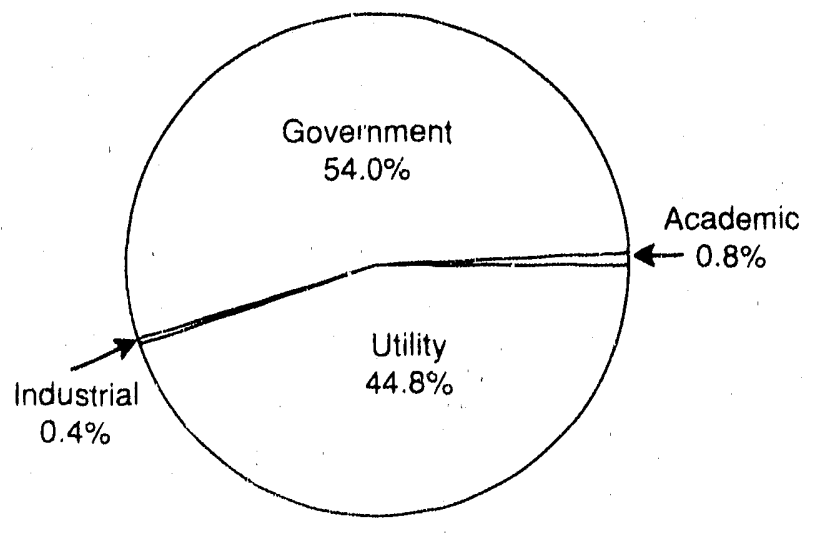

ACTIVITY PERCENTAGE BY SOURCE

(Percentages <. $1 \%$ are not displayed)

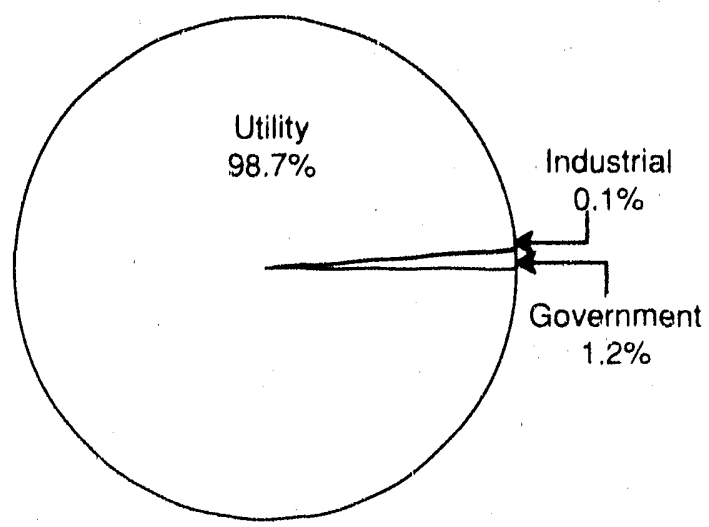




\section{MAINE \\ Low-Level Radioactive Wastes Received at Commercial Disposal Sites}

\begin{tabular}{c} 
Disposal \\
Site \\
\hline
\end{tabular}

Barnwell

Beatty

Richland

Total

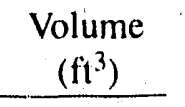

$15,579.90$

8.18

45.32

$15,633.40$
Activity

(curies)

273.53

0.21

0.04

273.78
VOLUME PERCENTAGE BY DISPOSAL SITE

(Percentages <. $1 \%$ are not displayed)

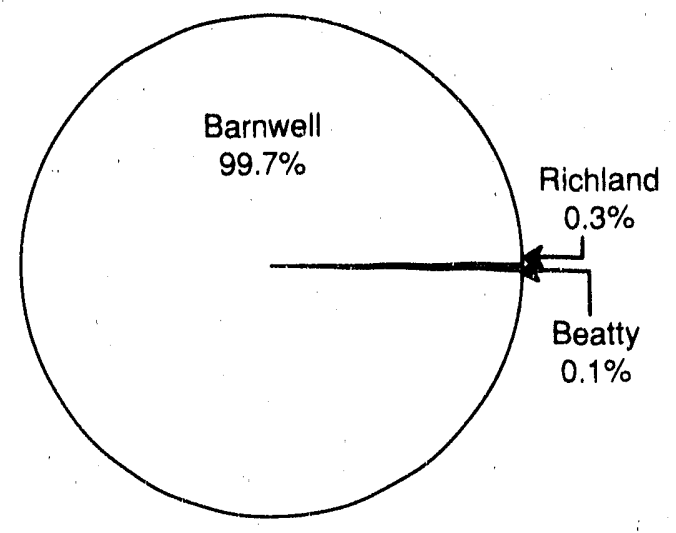

ACTIVITY PERCENTAGE BY DISPOSAL SITE (Percentages <.1\% are not displayed)

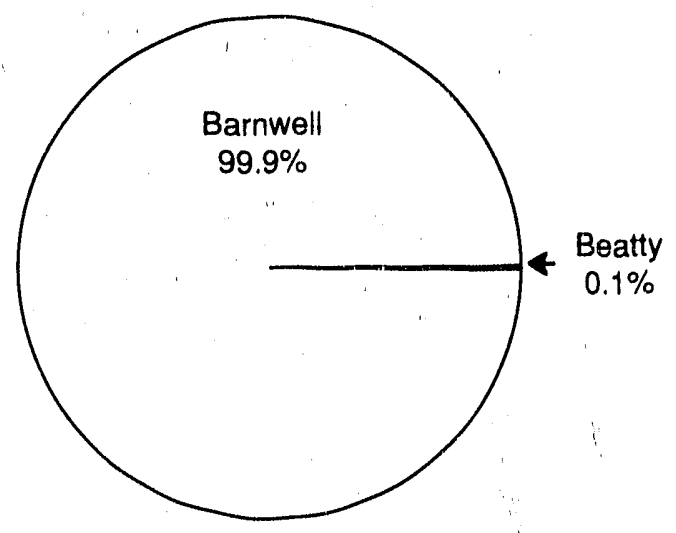

Volume Percentage by Waste Class for Direct Shipments and Total Non-Direct Volume Percentage (Percentages <. $1 \%$ are not displayed)
Direct Shipment

Waste Class

A
B
C

Total

Non-Direct Shipments

Grand Total

Total Direct Shipment

Activity (curies)

Total Non-Direct Shipment

Activity (curies)

Grand Total (curies)
Volume

$\left(\mathrm{ft}^{3}\right)$

$9,661.50$

372.20

188.60

$10,222.30$

$5,411.10$

$15,633.40$

264.73

9.05

273.78

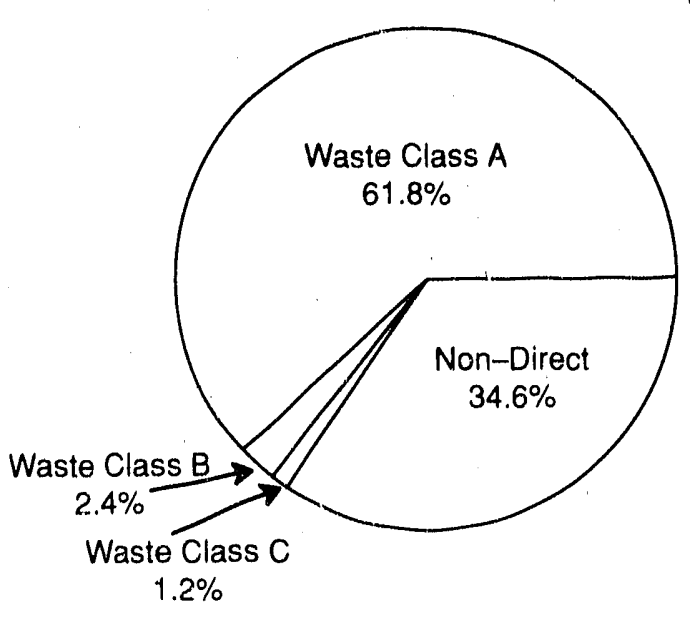




\section{MASSACHUSETTS \\ Low-Level Radioactive Wastes Received at Commercial Disposal Sites}

\begin{tabular}{l}
$\begin{array}{l}\text { Generator } \\
\text { Category }\end{array}$ \\
\hline Academic \\
Government \\
Industrial \\
Medical \\
Utility
\end{tabular}

Total

$$
\begin{array}{r}
\begin{array}{c}
\text { Volume } \\
\left(\mathrm{ft}^{3}\right)
\end{array} \\
\hline 734.18 \\
1,045.50 \\
34,590.27 \\
875.53 \\
19,281.59 \\
\hline 56,527.07
\end{array}
$$

$$
\begin{array}{r}
\begin{array}{l}
\text { Activity } \\
\text { (curies) }
\end{array} \\
\hline 14.41 \\
5.77 \\
57,067.25 \\
4.23 \\
294.05 \\
\hline 57,385.70
\end{array}
$$

VOLUME PERCENTAGE BY SOURCE (Percentages <. $1 \%$ are not displayed)

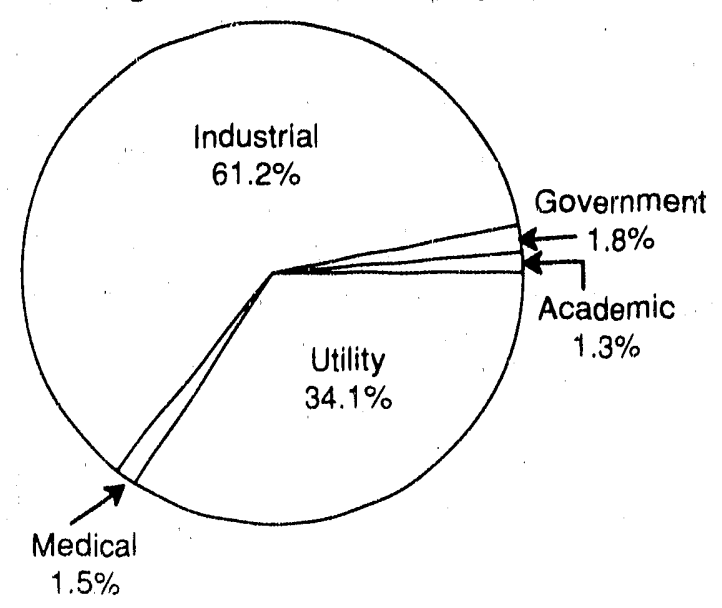

ACTIVITY PERCENTAGE BY SOURCE (Percentages <. $1 \%$ are not displayed)

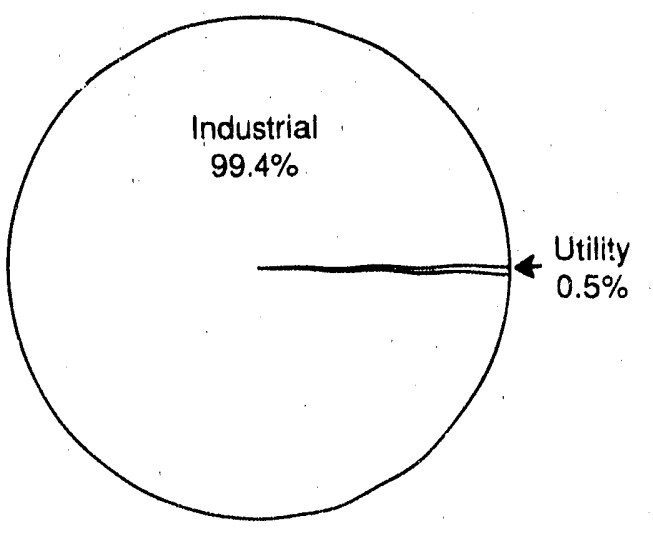




\section{MASSACHUSETTS \\ Low-Level Radioactive Wastes Received at Commercial Disposal Sites}

\begin{tabular}{|c|c|c|}
\hline $\begin{array}{c}\text { Disposal } \\
\text { Site } \\
\end{array}$ & $\begin{array}{c}\text { Volume } \\
\left(\mathrm{ft}^{3}\right)\end{array}$ & $\begin{array}{l}\text { Activity } \\
\text { (curies) }\end{array}$ \\
\hline Barnwell & $38,929.03$ & 718.14 \\
\hline Beatty & $6,635.73$ & 44.85 \\
\hline Richland & $10,962.31$ & $56,622.72$ \\
\hline Total & $56,527.07$ & $57,385.70$ \\
\hline
\end{tabular}

VOLUME PERCENTAGE BY DISPOSAL SITE (Percentages <. $1 \%$ are not displayed)

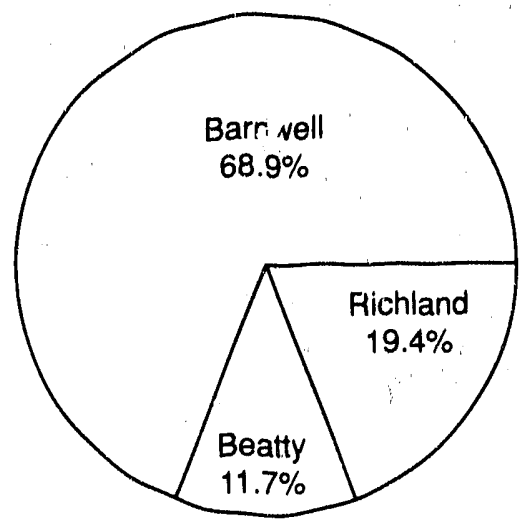

Direct Shipment

Waste Class

A

B

C

Total

Non-Direct Shipments

Grand Total

Total Direct Shipment

Activity (curies)

Total Non-Direct Shipment

Activity (curies)

Grand Total (curies)
Volume

$\left(\mathrm{ft}^{3}\right)$

$37,473.90$

$1,239.30$

0.00

$38,713.20$

$\underline{17,813.87}$

$56,527.07$

$57,251.32$

134.38

$57,385.70$
ACTIVITY PERCENTAGE BY DISPOSAL SITE

(Percentagas <.1\% are not displayed)

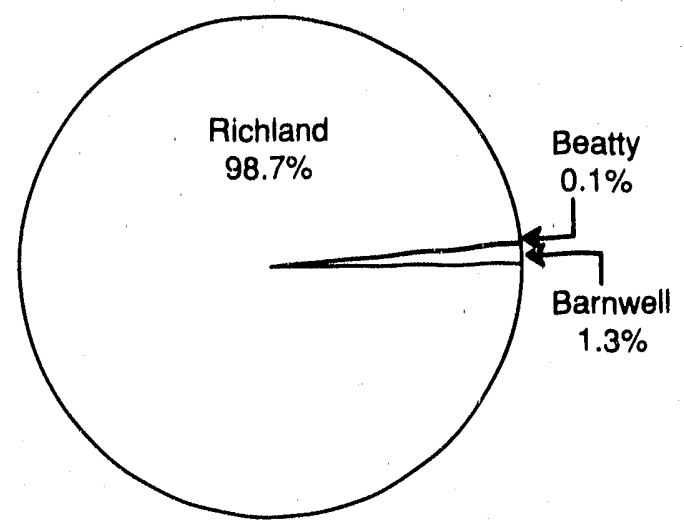

Volume Percentage by Waste Class for Direct Shipments and Total Non-Direct Volume Percentage (Percentages <.1\% are not displayed)

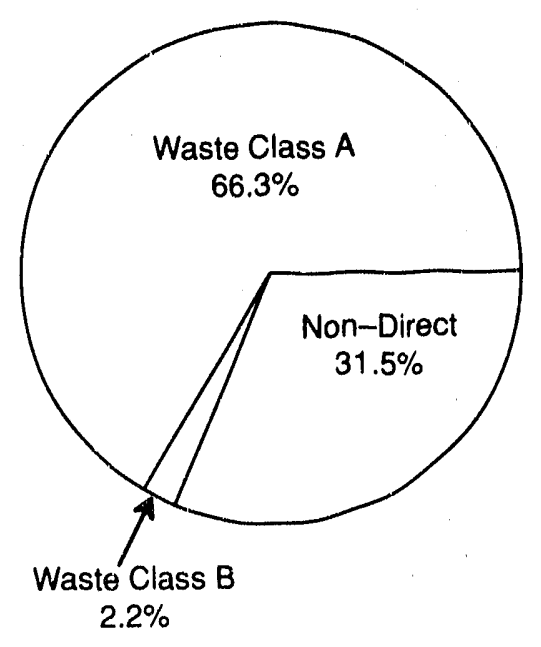




\section{NEW HAMPSHIRE \\ Low-Level Radioactive Wastes Received at Commercial Disposal Sites}

\section{Generator \\ Category}

Academic

Industrial

Medical

Total

\begin{tabular}{c}
$\begin{array}{c}\text { Volume } \\
\left(\mathrm{ft}^{3}\right)\end{array}$ \\
\hline
\end{tabular}

$22.4(1$

1.60

2.70

26.70
Activity

(curies)

$$
26.67
$$

$<1$

0.25

26.92
VOLUME PERCENTAGE BY SOURCE (Percentages <. $1 \%$ are not displayed)

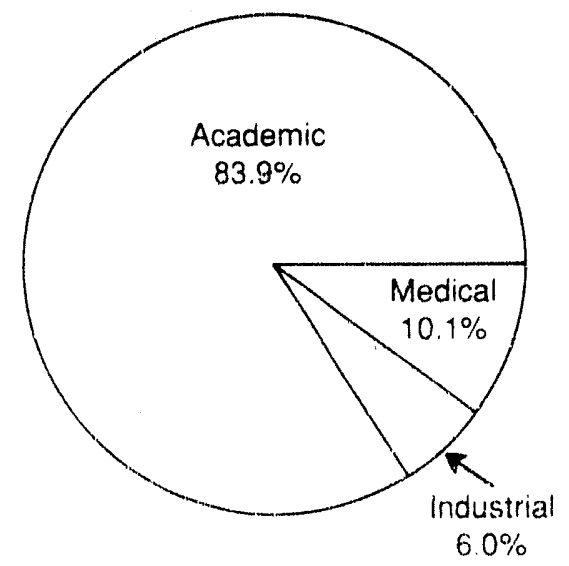

ACTIVITY PERCENTAGE BY SOURCE (Percentages <. $1 \%$ are not displayed)

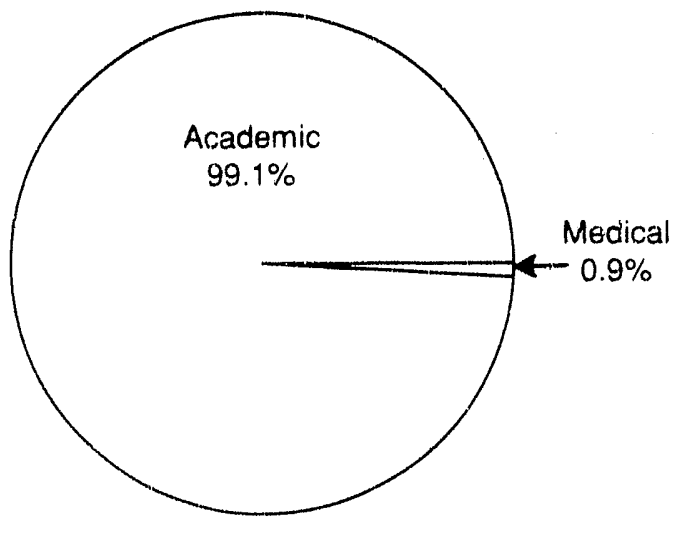




\section{NEW HAMPSHIRE \\ Low-Level Radioactive Wastes Received at Commercial Disposal Sites}

\begin{tabular}{c} 
Disposal \\
Site \\
\hline
\end{tabular}

Beatty

Total

$\begin{array}{r}\begin{array}{c}\text { Volume } \\ \left(\mathrm{ft}^{3}\right)\end{array} \\ \hline 26.70 \\ \hline 26.70\end{array}$

Activity

(curies)

26.92

26.92
VOLUME PERCENTAGE BY DISPOSAI. SITE (Percentages <.1\% are not clisplayed)

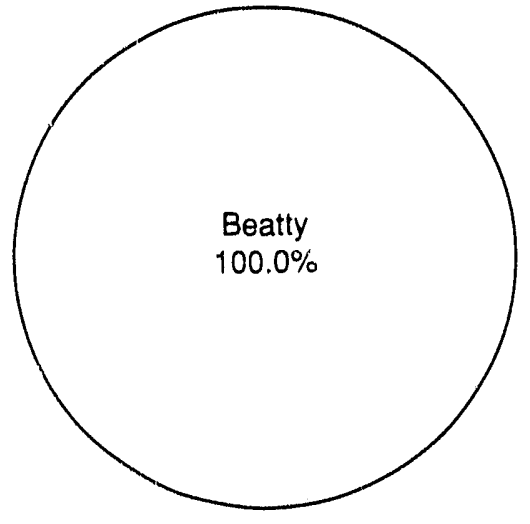

ACTIVITY PERCENTAGE BY DISPOSAL SITE

(Percentages <. $1 \%$ are not displayed)

\section{Volume Percentage by Waste Class \\ for Direct Shipments and Total}

Non-Direct Volume Percentage

(Percentages <. $1 \%$ are not displayed)

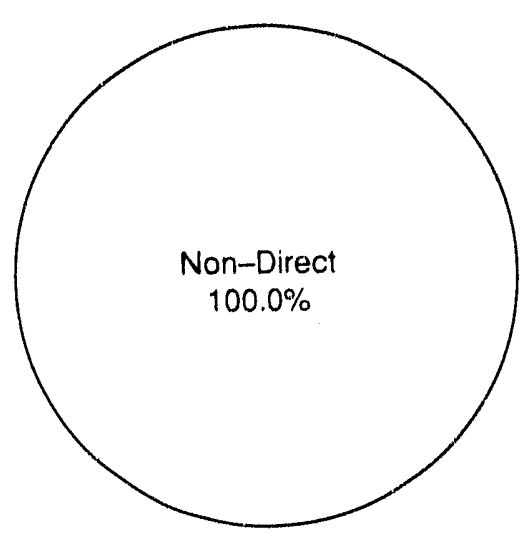




\section{NEW YORK \\ Low-Level Radioactive Wastes Received at Commercial Disposal Sites}

\begin{tabular}{l}
$\begin{array}{l}\text { Generator } \\
\text { Category }\end{array}$ \\
\hline Academic \\
Government \\
Industrial \\
Medical \\
Utility
\end{tabular}

Total

$$
\begin{array}{r}
\begin{array}{c}
\text { Volume } \\
\left(\mathrm{ft}^{3}\right)
\end{array} \\
\hline 2,713.18 \\
8,185.93 \\
10,587.04 \\
10,070.25 \\
65,336.98 \\
\hline
\end{array}
$$

$96,893.38$
Activity

(curies)

$$
\begin{array}{r}
1,017.03 \\
27.79 \\
4,848.58 \\
18.57 \\
95,541.88 \\
\hline 101,453.86
\end{array}
$$

VOLUME PERCENTAGE BY SOURCE (Percentages $<.1 \%$ are not displayed)

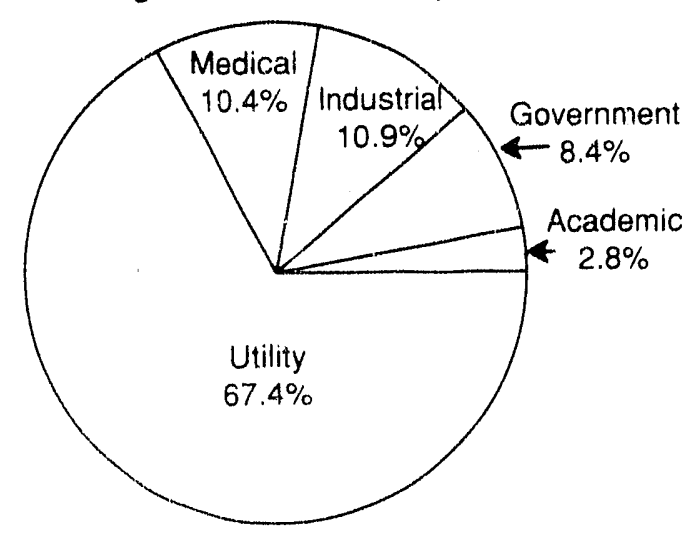

ACTIVITY PERCENTAGE BY SOURCE (Percentages <. $1 \%$ are not displayed)

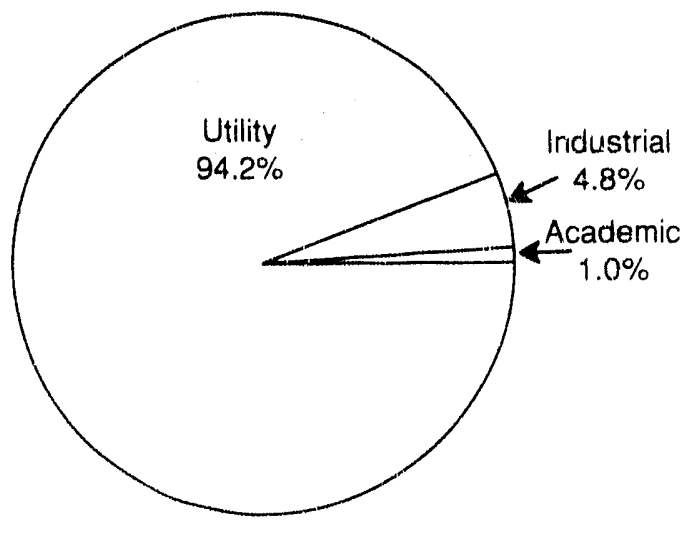




\section{NEW YORK \\ Low-Level Radioactive Wastes Received at Commercial Disposal Sites}

\begin{tabular}{|c|c|c|}
\hline $\begin{array}{l}\text { Disposal } \\
\text { Site }\end{array}$ & $\begin{array}{c}\text { Volume } \\
\left(\mathrm{ft}^{3}\right)\end{array}$ & $\begin{array}{l}\text { Activity } \\
\text { (curies) }\end{array}$ \\
\hline Barnwell & $68,649.96$ & $99,973.31$ \\
\hline Beatty & $3,064.77$ & $1,050.74$ \\
\hline Richland & $25,178.65$ & 429.81 \\
\hline Total & $96,893.38$ & $101,453.86$ \\
\hline
\end{tabular}

VOLUME PERCENTAGE BY DISPOSAL SITE

(Percentages <.1\% are not displayed)

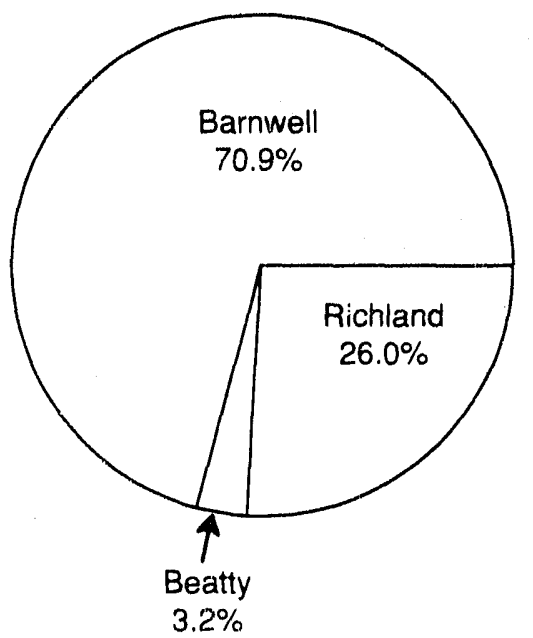

Direct Shipment

Waste Class

A

B

C

Total

Non-Direct Shipments

Grand Total

Total Direct Shipment

Activity (curies)

Total Non-Direct Shipment

Activity (curies)

Grand Total (curies) $\left(\mathrm{ft}^{3}\right)$
ACTIVITY PERCENTAGE BY DISPOSAL SITE (Percentages <.1\% are not displayed)

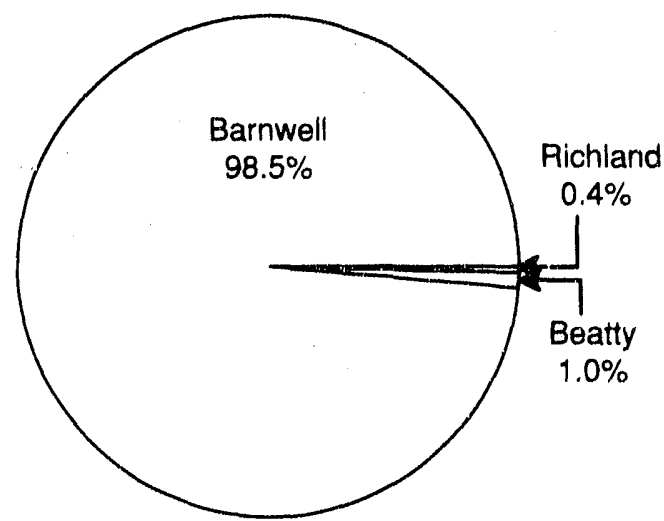

Volume Percentage by Waste Class for Direct Shipments and Total Non-Direct Volume Percentage (Percentages <. $1 \%$ are not displayed)

Volume

$40,226.50$

$3,482.20$ 529.50 Waste $3.6 \%$ $44,238.20$ $52,655.18$ $96,893.38$

$100,266.55$ $1,187.31$ $101,453.86$ 


\section{RHODE ISLAND \\ Low-Level Radioactive Wastes Received at Commercial Disposal Sites}

\section{Cenerator \\ Caiegory}

Academic

Government

Industrial

Medical

Total

$\begin{array}{r}\begin{array}{c}\text { Volume } \\ \left(\mathrm{ft}^{3}\right)\end{array} \\ \hline 402.61 \\ 0.50 \\ 43.53 \\ 972.67 \\ \hline\end{array}$

$1,419.31$
Activity

(curies)

$\begin{array}{r}0.05 \\ 8.49 \\ 13.67 \\ 0.49 \\ \hline 22.70\end{array}$

VOLUME PERCENTAGE BY SOURCE (Percentages <. $1 \%$ are not displayed)

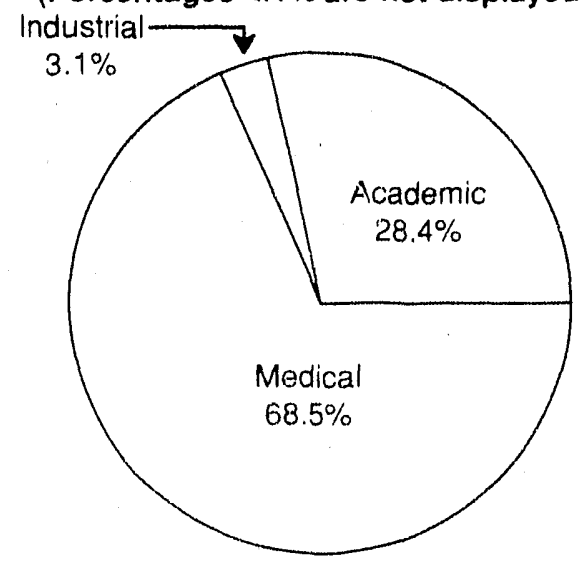

ACTIVITY PERCENTAGE BY SOURCE

(Percentages <. $1 \%$ are not displayed)

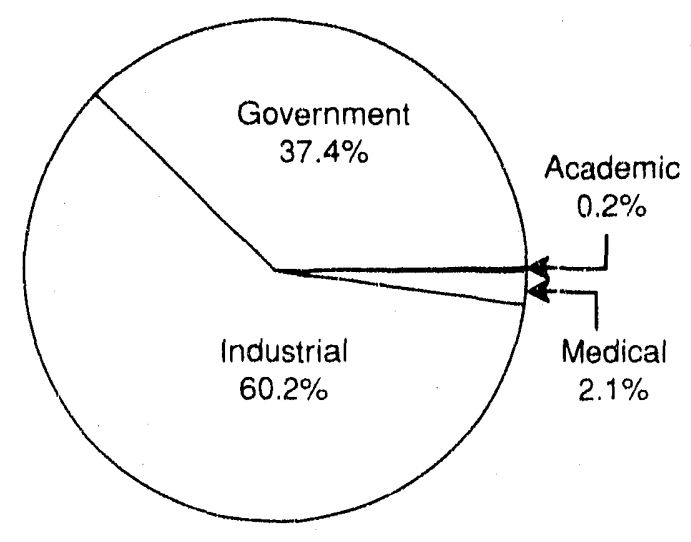




\section{RHODE ISLAND \\ Low-Level Radioactive Wastes Received at Commercial Disposal Sites}

\begin{tabular}{lr}
\multicolumn{1}{c}{$\begin{array}{c}\text { Disposal } \\
\text { Site }\end{array}$} & $\begin{array}{c}\text { Volume } \\
\left(\mathrm{ft}^{3}\right)\end{array}$ \\
Barnwell & $\begin{array}{r}741.70 \\
\text { Beatty }\end{array}$ \\
Richland & $\begin{array}{l}236.61 \\
441.00\end{array}$ \\
Total & $1,419.31$
\end{tabular}

Activity

(curies)

41.70

8.59

13.47

0.63

22.70

VOLUME PERCENTAGE BY DISPOSAL SITE

(Percentages <. $1 \%$ are not displayed)

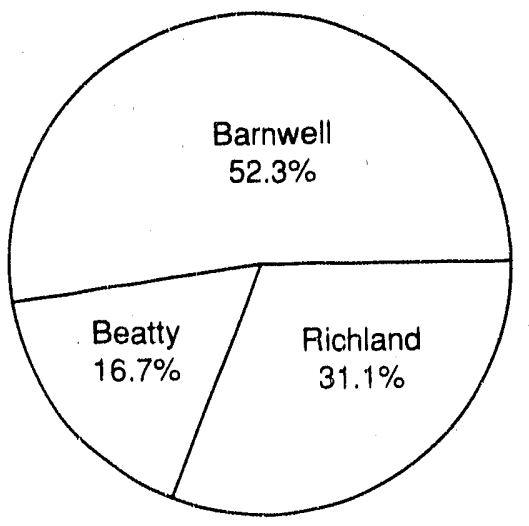

ACTIVITY PERCEI'TAGE BY DISPOSAL SITE

(Percentages, <. $1 \%$ are not displayed)

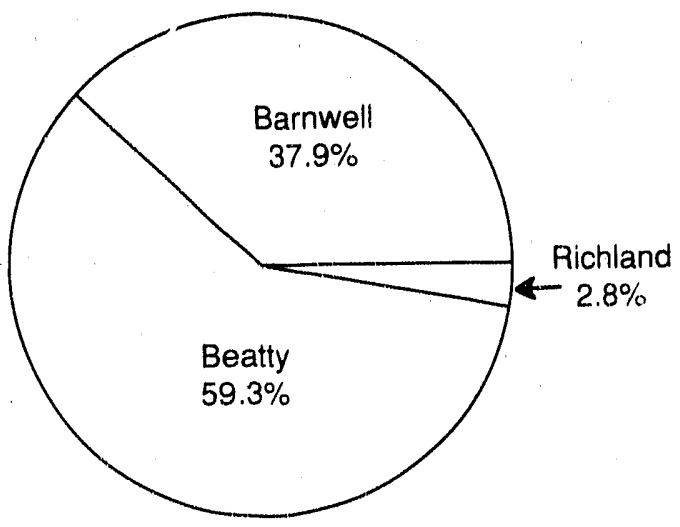

Volume Percentage by Waste Class for Direct Shipments and Total Non-Direct Volume Percentage

(Percentages <. $1 \%$ are not displayed)

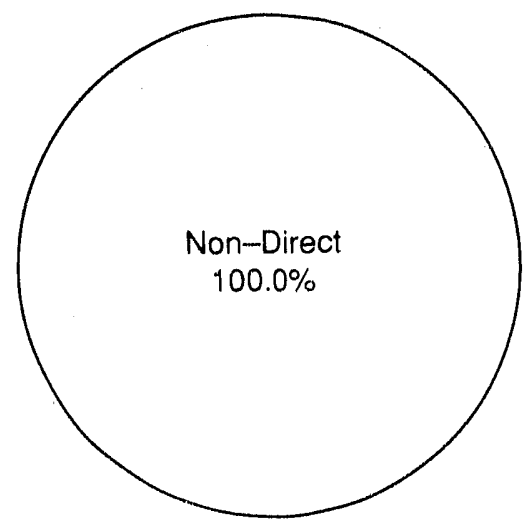


TEXAS

Low-Level Radioactive Wastes Received

at Commercial Disposal Sites

\begin{tabular}{l} 
Generator \\
Category \\
\hline
\end{tabular}

Academic

Government

Industrial

Medical

Utility

Total

VOLUME PERCENTAGE BY SOURCE

(Percentages <. $1 \%$ are not displayed)

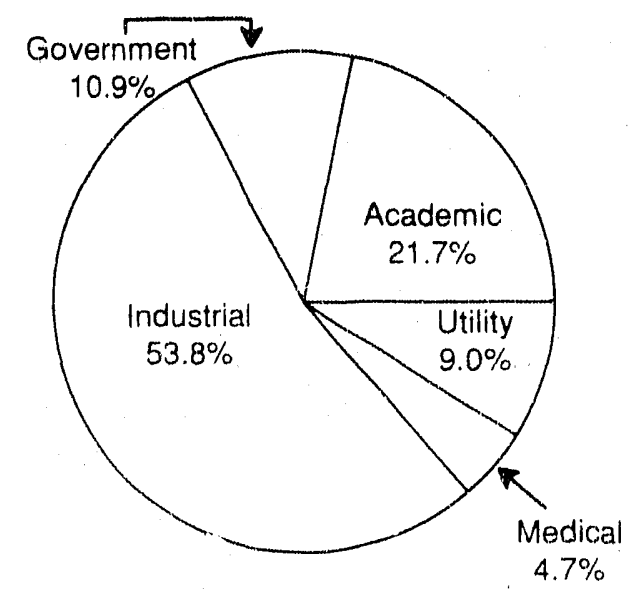

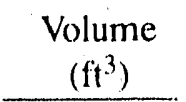

$2,404.80$

$11,916.32$

$1,051.41$

$1,984.50$

$22,157.48$

\section{$4,800.45$}

Activity

(curies)

63.42

127.65

$11,156.22$

2.25

7.49

$11,357.03$
AC'IIVITY PERCENTAGE BY SOURCE

(Percentages <.1\% are not displayed)

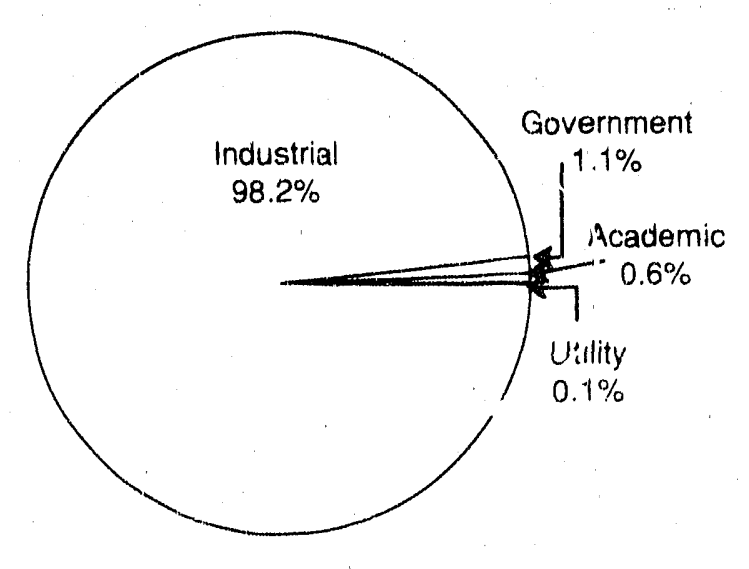




\section{TEXAS \\ Low-Level Radioactive Wastes Received at Commercial Disposal Sites}

Disposal
Site

Barnwell

Beatty

Richland

Total

\author{
Volume \\ $\left(\mathrm{ft}^{3}\right)$
}

$4,975.42$

$12,413.14$

$4,768.92$

$22,157.48$
Activity

(curies)

136.24

$11,183.88$

36.90

$11,357.03$

\section{VOLUME PERCENTAGE BY DISPOSAL SITE} (Percentages <.1\% are not displayed)

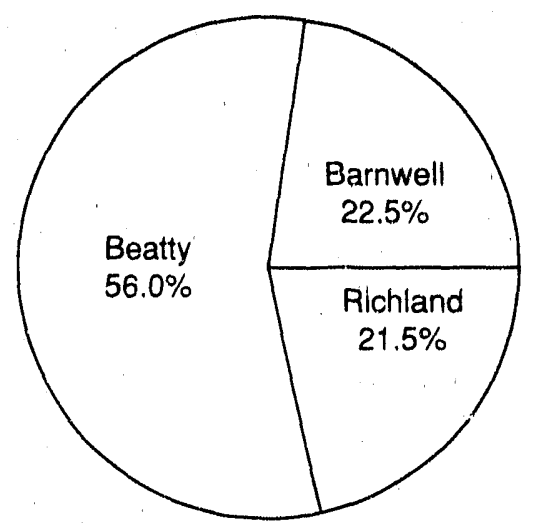

\section{ACTIVITY PERCENTAGE BY DISPOSAL SITE} (Percentages $<.1 \%$ are not displayed)

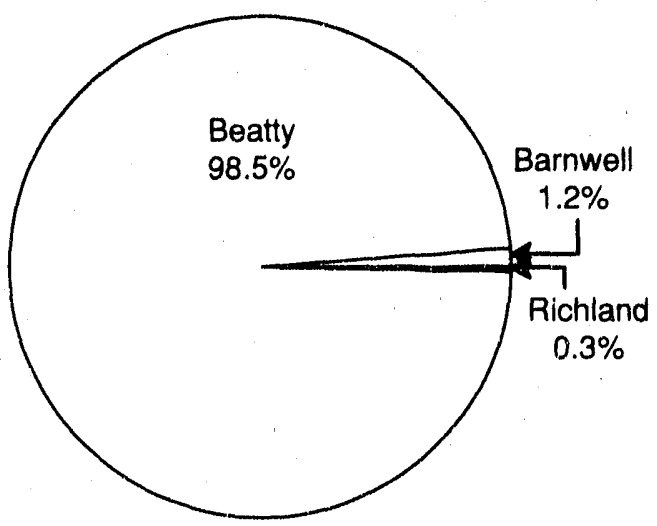

Volume Percentage by Waste Class for Direct Shipments and Total Non-Direct Volume Percentage (Percentages <. $1 \%$ are not displayed)
Direct Shipment

Waste Class

A

B

C

Total

Non-Direct Shipments

Grand Total

Total Direct Shipment

Activity (curies)

Total Non-Direct Shipment

Activity (curies)

Grand Total (curies)
Volume

$10,861.70$

37.50

15.00 Waste Class B

$10,914.20$

$11,243.28$

$22,157.48$

$10,236.36$

$1,120.66$

$11,357.03$

$0.2 \%$

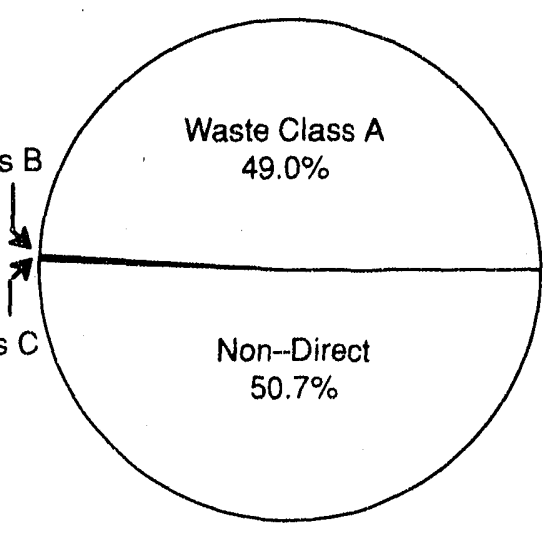




\section{VERMONT \\ Low-Level Radioactive Wastes Received at Commercial Disposal Sites}

\begin{tabular}{l} 
Generator \\
Category \\
\hline
\end{tabular}

Academic

Utility

Total
Volume

$\left(\mathrm{ft}^{3}\right)$

2.40

170.80

173.20
Activity

(curies)

$<1$

2.15
VOLUME PERCENTAGE BY SOURCE (Percentages <. $1 \%$ are not displayed)

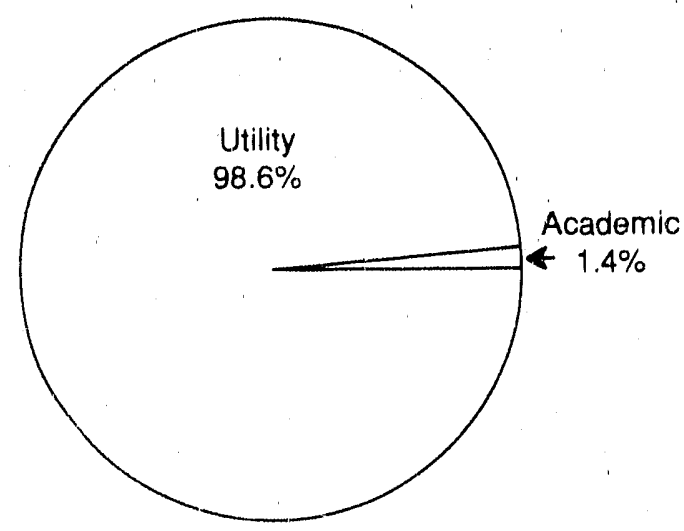

ACTIVITY PERCENTAGE BY SOURCE

(Percentages <. $1 \%$ are not displayed)

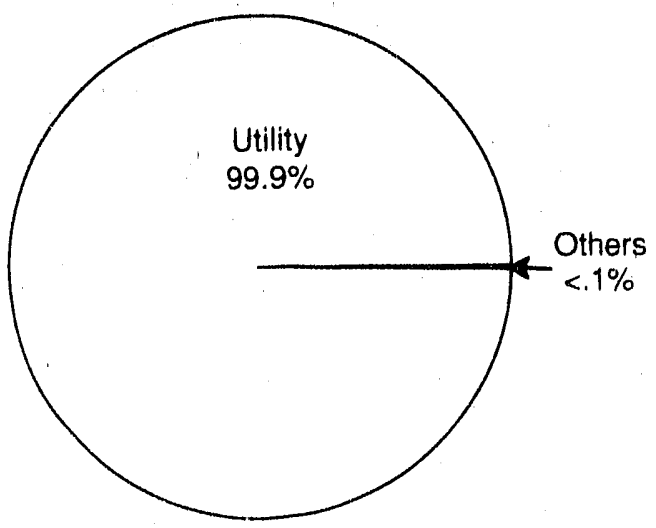




\section{VERMONT \\ Low-Level Radioactive Wastes Received at Commercial Disposal Sites}

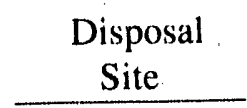

Barnwell

Total

\author{
Volume \\ $\left(\mathrm{ft}^{3}\right)$
}

173.20

173.20
Activity

(curies)

2.15

2.15
VOLUME PERCENTAGE BY DISPOSAL SITE

(Percentages <. $1 \%$ are not displayed)

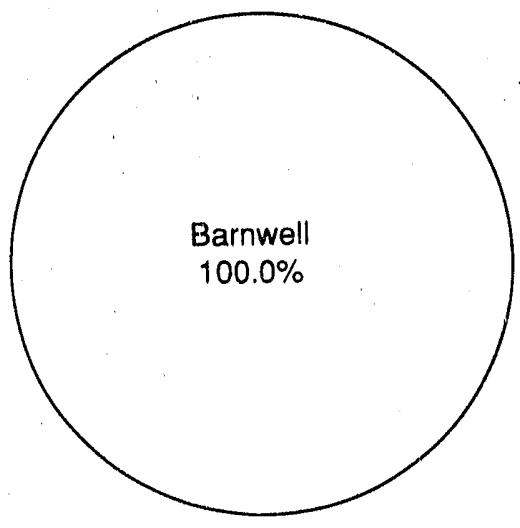

ACTIVITY PERCENTAGE BY DISPOSAL SITE

(Percentages <. $1 \%$ are not displayed)

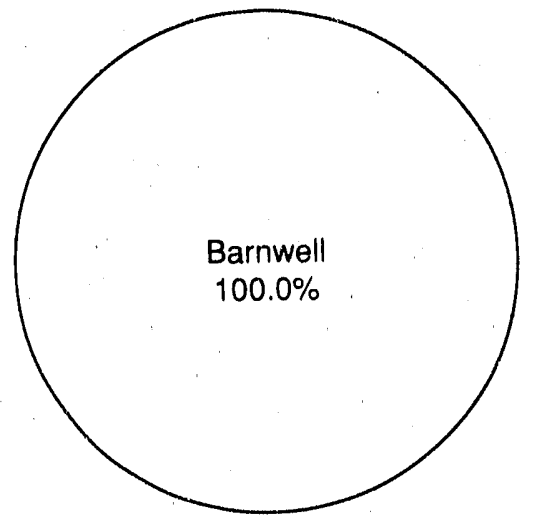

Volume Percentage by Waste Class for Direct Shipments and Total Non-Direct Volume Percentage (Percentages <.1\% are not displayed)
Direct Shipment

Waste Class

$$
\begin{aligned}
& \text { A } \\
& \text { B } \\
& \text { C }
\end{aligned}
$$

Total

Non-Direct Shipments

Grand Total

Total Direct Shipment

Activity (curies)

Total Non-Direct Shipment

Activity (curies)

Grand Total (curies)
Volume

$\left(\mathrm{ft}^{3}\right)$

170.80

0.00

0.00

170.80

2.40

173.20

2.15

$\underline{0.00}$

2.15

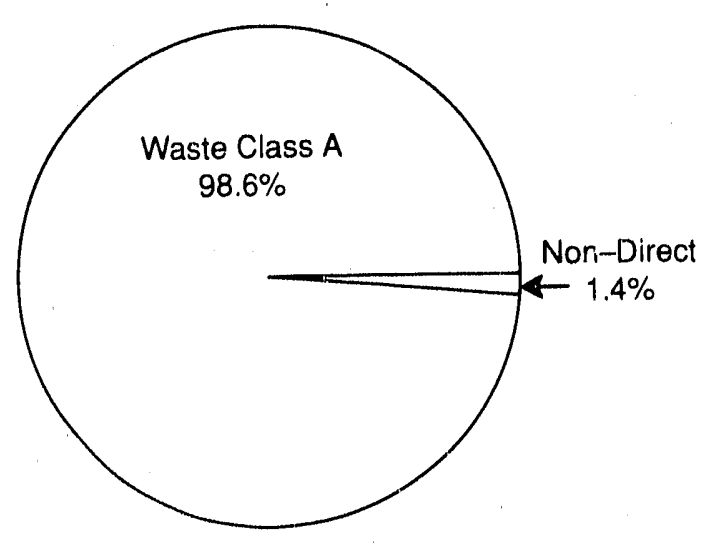




\section{APPENDIX B \\ WASTE VOLUME COMPARISON BY STATE \\ AND \\ COMMERCIAL NUCLEAR POWER REACTORS \\ IN THE UNITED STATES}


Table B-1. Waste Volume Comparison by State, 1985-1989, as Reported by Disposal Site Operators

\begin{tabular}{|c|c|c|c|c|c|}
\hline \multirow[b]{2}{*}{ State } & \multicolumn{5}{|c|}{$\begin{array}{r}\text { Volume } \\
\left(\mathrm{ft}^{3}\right) \\
\end{array}$} \\
\hline & 1985 & 1986 & 1987 & 1988 & 1989 \\
\hline Alabama & 102,472 & 58,221 & 70,212 & 50,949 & 49,249 \\
\hline Alaska & 0 & 0 & 0 & 4 & 11 \\
\hline Arizona & 3,647 & 4,789 & 16,601 & 28,345 & 31,756 \\
\hline Arkansas & 25,408 & 4,474 & 19,628 & 7,033 & 7,970 \\
\hline California & 250,882 & 114,011 & 99,304 & 83,157 & 117,966 \\
\hline Colorado & 10,202 & 1,073 & 3,586 & 2,104 & 8,754 \\
\hline Connecticut & 62,653 & 55,700 & 40,445 & 41,890 & 49,078 \\
\hline Delaware & 1,208 & 1,473 & 1,404 & 1,420 & 1,377 \\
\hline District of Columbia & 1,051 & 230 & 135 & 980 & 925 \\
\hline Florida & 59,131 & 60,109 & 46,079 & 31,027 & 35,283 \\
\hline Georgia & 78,299 & 48,275 & 29,615 & 38,940 & 44,697 \\
\hline Hawaii & 11,478 & 2,029 & 2,598 & 3,943 & 6,204 \\
\hline Idaho & 1,350 & 0 & 2 & 51 & 138 \\
\hline Illinois & 360,383 & 227,171 & 190,224 & 112,529 & 134,787 \\
\hline Indiana & 1,127 & 0 & 1,808 & 1,892 & 2,150 \\
\hline lowa & 29,592 & 9,973 & 18,646 & 7,207 & 6,055 \\
\hline Kansas & 1,700 & 6,575 & 4,506 & 5,015 & 6,365 \\
\hline Kentucky & 3,583 & 3,790 & 176 & 2,122 & 10,047 \\
\hline Louisiana & 10,979 & 22,534 & 27,826 & 18,401 & 21,723 \\
\hline Maine & 13,053 & 8,324 & 5,013 & 6,338 & 15,633 \\
\hline Maryland & 38,304 & 18,856 & 19,637 & 30,604 & 39,996 \\
\hline Massachusetts & 105,565 & 66,695 & 55,023 & 51,273 & 56,527 \\
\hline Michigan & 55,110 & 36,234 & 35,284 & 27,136 & 43,111 \\
\hline Minnesota & 47,254 & 28,061 & 19,637 & 15,477 & 21,954 \\
\hline Mississippi & 22,028 & 18,625 & 14,119 & 18,173 & 10,996 \\
\hline Missouri & 11,006 & 24,497 & 22,858 & 11,912 & 18,803 \\
\hline Montana & 87 & 591 & 38 & 14 & 107 \\
\hline Nebraska & 37,427 & 20,342 & 17,409 & 12,822 & 16,699 \\
\hline Nevada & 62 & 0 & 15 & 668 & 308 \\
\hline New Hampshire & 1,755 & 2,109 & 394 & 169 & 27 \\
\hline New Jersey & 66,395 & 55,521 & 50,292 & 46,371 & 53,540 \\
\hline New Mexico & 2,663 & 0 & 990 & 304 & 1,369 \\
\hline New York & 161,185 & 106,547 & 70,409 & 73,069 & 96,893 \\
\hline North Carolina & 102,463 & 81,847 & 78,791 & 62,446 & 58,422 \\
\hline North Dakota & 0 & 0 & 3 & 0 & 105 \\
\hline Ohio & 34,090 & 16,052 & 18,662 & 22,585 & 58,866 \\
\hline Oklahoma & 10,536 & 49,815 & 82,855 & 28,446 & 32,127 \\
\hline Oregon & 58,247 & 108,983 & 82,150 & 83,796 & 75,908 \\
\hline
\end{tabular}


Table B-1. (continued)

\begin{tabular}{|c|c|c|c|c|c|}
\hline \multirow[b]{2}{*}{ State } & \multicolumn{5}{|c|}{$\begin{array}{r}\text { Volume } \\
\left(\mathrm{ft}^{3}\right) \\
\end{array}$} \\
\hline & 1985 & 1986 & 1987 & 1988 & 1989 \\
\hline Pennsylvania & 262,099 & 188,038 & 144,798 & 150,298 & 129,485 \\
\hline Puerto Rico & 0 & 0 & 0 & 0 & 0 \\
\hline Rhode Island & 1,104 & 1,210 & 1,045 & 1,108 & 1,419 \\
\hline South Carolina & 136,130 & 121,134 & 118,060 & 95,168 & 96,695 \\
\hline South Dakota & 8 & 8 & 0 & 0 & 1 \\
\hline Tennessee : & 237,250 & 81,431 & 238,779 & 119,051 & 118,957 \\
\hline Texas & 10,565 & 4,376 & 69,201 & 12,064 & 22,158 \\
\hline Utah & 5,213 & 3,405 & 2,715 & 4,672 & 6,299 \\
\hline Vermont & 19,966 & 11,753 & 8,263 & 7,248 & 173 \\
\hline Virginia & 146,769 & 71,196 & 67,985 & 64,129 & 82,834 \\
\hline Washington & 62,584 & 52,987 & 38,244 & 35,896 & 26,409 \\
\hline West Virginia & 420 & 2 & 5 & 148 & 411 \\
\hline Wisconsin & 16,085 & 5,938 & 9,035 & 10,599 & 6,881 \\
\hline Wyoming & 0 & 0 & 0 & 0 & 1 \\
\hline Army out U.S. & $\mathrm{N} / \mathrm{R}$ & $\mathrm{N} / \mathrm{R}$ & $\mathrm{N} / \mathrm{R}$ & 3 & 164 \\
\hline Total & $2,680,635$ & $1,804,999$ & $1,844,504$ & $1,429,016$ & $1,627,813$ \\
\hline
\end{tabular}


Table B-2. Commercial Nuclear Power Reactors in the United States, as of December 31, 1989a

\begin{tabular}{|c|c|c|c|}
\hline State Name & Facility Name & Units & Type \\
\hline \multirow[t]{2}{*}{ Alabama } & Browns Ferry Nuclear Power Station & $1,2 \& 3$ & $\mathrm{BWR}^{\mathrm{b}}$ \\
\hline & Joseph M. Farley Nuclear Plant & $1 \& 2$ & $\mathrm{PWR}^{\mathrm{c}}$ \\
\hline Arizona & Palo Verde Nuclear Generating Station & $1,2 \& z 3$ & PWR \\
\hline Arkansas & Arkansas Nuclear One & $1 \& 2$ & PWR \\
\hline \multirow[t]{2}{*}{ California } & Diablo Canyon Nuclear Power Plant & $1 \& 2$ & PWR \\
\hline & Humboldt Bay Power Plant (closer: 1976) & 3 & BWR \\
\hline & $\begin{array}{l}\text { Rancho Seco Nuclear Generating Sitation } \\
\text { (closed June 1989) }\end{array}$ & 1 & PWR \\
\hline & San Onofre Nuclear Generating Station & $1,2 \& 3$ & PWR \\
\hline Colorado & $\begin{array}{l}\text { Ft. St. Vrain Nuclear Generating Station } \\
\text { (closed August 1989) }\end{array}$ & 1 & HTGR $^{d}$ \\
\hline \multirow[t]{3}{*}{ Connecticut } & Haddam Neck Plant & 1 & PWR \\
\hline & Millstone Nuclear Power Station & 1 & BWR \\
\hline & Millstone Nuclear Power Station & $2 \& 3$ & PWR \\
\hline \multirow[t]{3}{*}{ Florida } & Crystal River Nuclear Plant & 3 & PWR \\
\hline & St. Lucie Plant & $1 \& 2$ & PWR \\
\hline & Turkey Point Plant & $3 \& 4$ & PWR \\
\hline \multirow[t]{2}{*}{ Georgia } & Edwin I. Hatch Nuclear Plant & $1 \& 2$ & BWR \\
\hline & Alvin W. Vogtle Jr. Nuclear Plant & $1 \& 2$ & PWR \\
\hline \multirow[t]{7}{*}{ Illinois } & Byron Nuclear Power Station & $1 \& 2$ & PWR \\
\hline & Clinton Power Station & 1 & BWR \\
\hline & $\begin{array}{l}\text { Dresden Nuclear Power Station (Unit } 1 \\
\text { closed 1978) }\end{array}$ & $1,2 \& 3$ & BWR \\
\hline & LaSalle County Station & $1 \& 2$ & BWR \\
\hline & Quad Cities Station & $1 \& 2$ & BWR \\
\hline & Zion Nuclear Plant & $1 \& 2$ & PWR \\
\hline & Braidwood Station & $1 \& 2$ & PWR \\
\hline Iowa & Duane Arnold Energy Center & 1 & BWR \\
\hline Kansas & Wolf Creek Generating Station & 1 & PWR \\
\hline \multirow[t]{2}{*}{ Louisiana } & River Bend Station & 1 & BWR \\
\hline & Waterford Generating Station & 3 & PWR \\
\hline
\end{tabular}


Table B-2. (continued)

\begin{tabular}{|c|c|c|c|}
\hline State Name & Facility Name & Units & Type \\
\hline Maine & Maine Yankee Atomic Power Plant & 1 & PWR \\
\hline Maryland & Calvert Cliffs Nuclear Power Plant & $1 \& 2$ & PWR \\
\hline \multirow[t]{2}{*}{ Massachusetts } & Pilgrim Nuclear Power Station & 1 & BWR \\
\hline & Yankee Nuclear Power Station & 1 & PWR \\
\hline \multirow[t]{4}{*}{ Michigan } & Big Rock Point Nuclear Plant & 1 & BWR \\
\hline & Donald C. Cook Nuclear Power Plant & $1 \& 2$ & PWR \\
\hline & Fermi Atomic Power Plant & 2 & BWR \\
\hline & Palisades Nuclear Plant & 1 & PWR \\
\hline \multirow[t]{2}{*}{ Minnesota } & Monticello Nuclear Generating Plant & 1 & BWR \\
\hline & Prairie Island Nuclear Generating Plant & $1 \& 2$ & PWR \\
\hline Mississippi & Grand Gulf Nuclear Station & 1 & $B W / R$ \\
\hline Missouri & Callaway Plant & 1 & PWR \\
\hline \multirow[t]{2}{*}{ Nebraska } & Cooper Nuclear Station & 1 & BWR \\
\hline & Fort Calhoun Station & 1 & PWR \\
\hline \multirow[t]{3}{*}{ New Jersey } & Hope Creek Nuclear Generating Station & 1 & BWR \\
\hline & Oyster Creek Nuclear Power Plant & 1 & BWR \\
\hline & Salem Nuclear Generating Station & $1 \& 2$ & PWR \\
\hline \multirow[t]{6}{*}{ New York } & James A. FitzPatrick Nuclear Power Plant & 1 & BWR \\
\hline & Robert Emmett Ginna Nuclear Power Plant & 1 & PWR \\
\hline & Indian Point Station & 3 & PWR \\
\hline & Indian Point Station (Unit 1 closed 1974) & $1 \& 2$ & PWR \\
\hline & Nine Mile Point Nuclear Station & $1 \& 2$ & BWR \\
\hline & $\begin{array}{l}\text { Shoreham Nuclear Power Station } \\
\text { (closed May 1989) }\end{array}$ & 1 & BWR \\
\hline \multirow[t]{3}{*}{ North Carolina } & Brunswick Steam Electric Plant & $1 \& 2$ & BWR \\
\hline & McGuire Nuclear Station & $1 \& 2$ & PWR \\
\hline & Shearon Harris Nuclear Power Plant & 1 & PWR \\
\hline \multirow[t]{2}{*}{ Ohio } & Davis-Besse Nuclear Power Station & 1 & PWR \\
\hline & Perry Nuclear Power Plant & 1 & BWR \\
\hline Oregon & Trojan Nuclear Plant & 1 & PWR \\
\hline
\end{tabular}


Table B-2. (continued)

\begin{tabular}{|c|c|c|c|}
\hline State Name & Facility Name & Units & Type \\
\hline \multirow[t]{5}{*}{ Pennsylvania } & Beaver Valley Power Station & $1 \& 2$ & PWR \\
\hline & Limerick Generating Station & 1 & BWR \\
\hline & Peach Bottom Atomic Power Station & $2 \& 3$ & BWR \\
\hline & Susquehanna Steam Electric Station & $1 \& 2$ & BWR \\
\hline & Three Mile Island Nuclear Station & 1 & PWR \\
\hline \multirow[t]{4}{*}{ South Carolina } & Catawba Nuclear Station & $1 \& 2$ & PWR \\
\hline & Oconee Nuclear Plant & $1,2, \& 3$ & PWR \\
\hline & Robinson Plant & 2 & PWR \\
\hline & Virgil C. Summer Nuclear Station & 1 & PWR \\
\hline Tennessee & Sequoyah Nuclear Plant & $1 \& 2$ & PWR \\
\hline Texas & South Texas Project & $1 \& 2$ & PWR \\
\hline Vermont & Vermont Yankee Nuclear Power Station & 1 & BWR \\
\hline \multirow[t]{2}{*}{ Virginia } & North Anna Power Station & $1 \& 2$ & PWR \\
\hline & Surry Power Station & $1 \& 2$ & PWR \\
\hline Washington & Washington Nuclear Project & 2 & BWR \\
\hline \multirow[t]{3}{*}{ Wisconsin } & Kewaunee Nuclear Power Plant & 1 & PWR \\
\hline & $\begin{array}{l}\text { La Crosse Nuclear Generating Station } \\
\text { (closed 1987) }\end{array}$ & 1 & BWR \\
\hline & Point Beach Nuclear Plant & $1 \& 2$ & PWR \\
\hline \multicolumn{4}{|c|}{ a. "World List of Nuclear Power Plants," Nuclear News, 33, 10, August 199(). } \\
\hline \multicolumn{4}{|c|}{ b. $\mathrm{BWR}=$ boiling-water reactor. } \\
\hline \multicolumn{4}{|c|}{ c. $P W R=$ pressurized-water reactor. } \\
\hline $\mathrm{d} H \mathrm{HT}$ & reactor. & & \\
\hline
\end{tabular}




\section{APPENDIX C \\ STATE RADIATION PROGRAM REPRESENTATIVES}

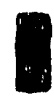

$\because$

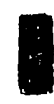

$\theta$

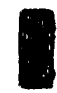

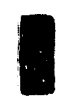

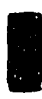

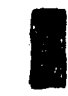

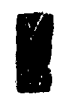

$\square$ 
Table C-1. State Radiation Program Representatives

\begin{tabular}{|c|c|}
\hline State & Draft Report Sent to \\
\hline Alabama & A. V. Godwin \\
\hline Alaska & S. D. Heidersdorf \\
\hline Arizona & C. F. Tedford \\
\hline Arkansas & G. J. Discus \\
\hline California & J. S. McGurk \\
\hline Colorado & R. M. Quillin \\
\hline Connecticut & K. T. A. McCarthy \\
\hline Delaware & A. C. Tapert \\
\hline Dist. of Columbia & F. A. Bowie \\
\hline Florida & M. E. Clark \\
\hline Georgia & T. E. Hill \\
\hline Hawaii & T. Anamizu \\
\hline Idaho & E. Ranieri \\
\hline Illinois & P. Eastvold \\
\hline Indiana & E. Wroblewski \\
\hline lowa & D. A. Flater \\
\hline Kansas & G. W. Allen \\
\hline Kentucky & D. R. Hughes \\
\hline Louisiana & W. H. Spell \\
\hline Maine & C. Toppan \\
\hline Maryland & R. G. Fletcher \\
\hline Massachusetts & R. M. Hallisey \\
\hline Michigan & G. W. Bruchmann \\
\hline & $(-3)$ \\
\hline
\end{tabular}


Table C-1. (continued)

State

Minnesota

Mississippi

Missouri

Montana

Nebraska

Nevada

New Hampshire

New Jersey

New Mexico

New York

North Carolina

North Dakota

Ohio

Oklahoma

Oregon

Pennsylvania

Puerto Rico

Rhode Island

South Carolina

South Dakota

Tennessee

Texas
Draft Report Sent to
A. T. Dolezal
E. S. Fuente

K. V. Miller

A. C. Howe

H. R. Borchert

S. R. Marshall

D. E. Tefft

J. Lipoti

J. Ellvinger

P. J. Merges

D. H. Brown

D. K. Mount

R. E. Owen

W. M. Kemp

R. D. Paris

T. M. Gerusky

D. Saldana

C. McMahon

H. G. Shealy

J. Robertson

M. H. Mobley

D. K. Lacker 
Table C-1. (continued)

\begin{tabular}{ll}
\hline \multicolumn{1}{c}{ State } & \multicolumn{1}{c}{ Draft Report Sent to } \\
Utah & L. F. Anderson \\
Vermont & R. N. McCandless \\
Virginia & L.P. Foldesi \\
Washington & T. R. Strong \\
West Virginia & B. L. DeBord \\
Wisconsin & M. C. Bunge \\
Wyoming & J. E. Haes \\
\hline
\end{tabular}



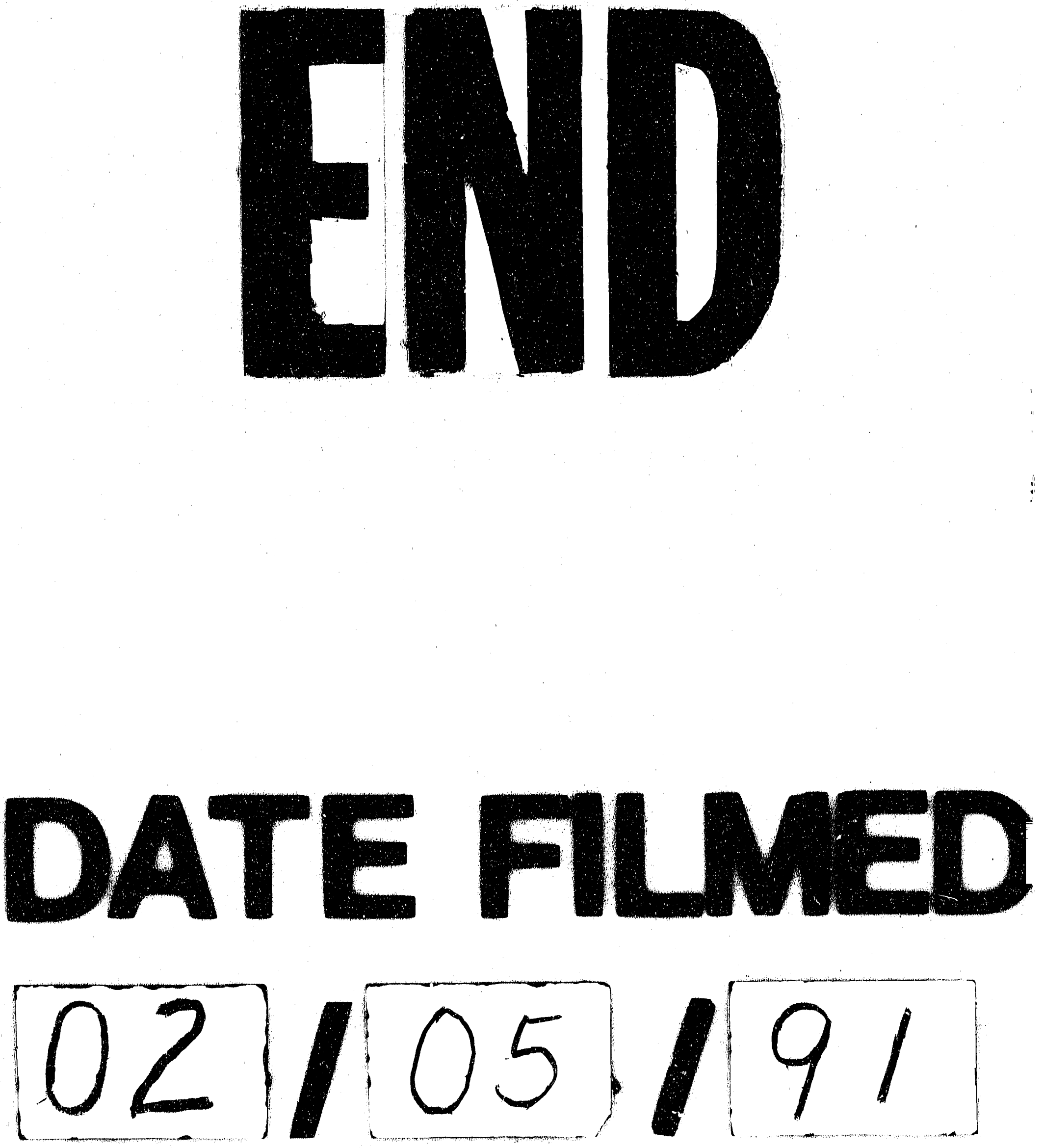
$\Xi$ 